





\section{POETI E PERSONAGGI CATULLIANI}

$\operatorname{Tan} .1439$ 



\section{CARLO PASCAL}

PROFESSORE NELLA R. LNIVERSITA DI PAVIA

\section{POETI E PERSONAGGI \\ CATULLIANI}

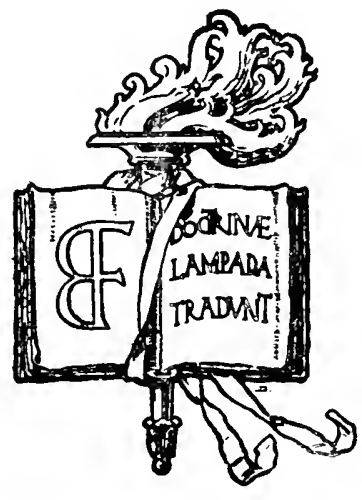

CATANIA

FRANCESCO BATTIATO - EDITORE

1916 


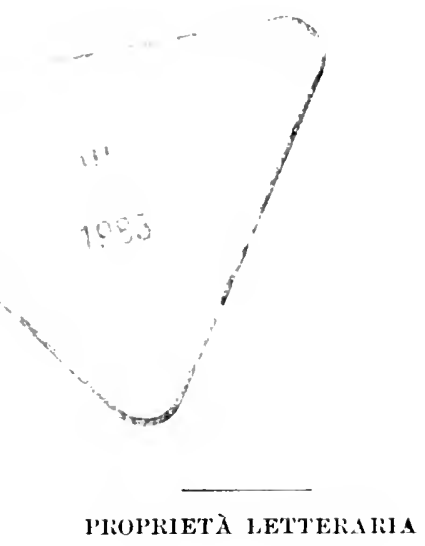

Catania - Stal. Tip. S. Di Mattel \& C. - 1916 


\section{AI IFTTORF}

Eccoti, amico lettore, nue serie di qnadri e di figure, che spiccano con maggiore riliero nei carmi catulliani, $e$ che sono in qualche modo rappresentativi delle tendenze letterarie, dei dibattiti politici, delle spensierateine mondane di quella etì. Di Licinio Calro e di Elvio Cinna, che furono veramente grandi poeti, il tempo tutte sommerse miseramente le opere, solo a noi conservando lacere reliquie, in alcune delle quali è dato ancor ravvisare graziose movenze poetiche, e fresche $e_{z}$ a di ispirasione immediata. Ma quei due poeti furono cosi intimamente congiunti con Catullo, per fuda amicisia, per indirizai poetici, per consentimenti politici, che a noi è possibile studiarli ed illnstrarli alla luce dell'arte catulliana, onde piu d'un raggio emana ad illuminarnc le figure. Non soffusi della medesima simpatica luce sono tutti gli altri personaggi illustrati nel nostro volume; ma, per essere i principali personaggi di Roma, formano tutti insieme un gran quadro: il quadro del- 
l'alta soriptir rmmana, rilta non nelle forme appariscenti e solemi, ma mendari e ingannatrici, della rita ufficiale. bersi nclle debole: $i e$ nei irii $e$ nelle segrete corruttele della rita pricata e familiare: una società che sotto lorpello delle grandiositi esteriori celava il tarlo roditore della sunc existenia e della suc poten:a: una società torbirla c intrigante, pettegola e malignu, spensierata ed aride di piaceri.

Il piccolo libretto di C'atullo non è solo un grande moummento di poesia, bensi anche un grande monumento li storia: e di ma storia che non è quclla solita, dei grandi fatti $e$ rei documenti nfficiali; ma è quella intima ed oscura e mimuta, dellc passioni, degli odii e degli rmori, she sono i morenti delle a:ioni, e ci rivelano dunpue di quellaltra storia le ragioni ignorate e profonde, e costituiscono nel loro insieme il quadro morale di ma societri. D'altra parte lo studio di tutti. questi elementi non poca luce imaggia sullarte stessa del poeta: giarrhè quell'urte non cra fredla, accadomira, astratta ed obliettiva: mu era forvila, commossa emanrsione della rita che il poeta riveva, dei suoi stcgni, dei suoi fremiti, e delle sue gelosie: della rita chc $\therefore$ agitarn intormo " lui, o della quale egli arcoglicra rellimima le impressioni profonde, che gli rimbalsarano poi, potenti e rigorose, nei carmi. Eccoti, amiro lettore, spricguto il fine di questo mio rolume, che in raccomando alla ture benevolemia.

Carlo pascal 


\section{A V VERTENZA}

Si notano qui sotto le principali edizioni di Catullo, citate per lo più nel corso del volume abbreviatamente, col semplice nome dell'editore.

Per le edizioni dei frammenti dei poeti catulliani, si rimanda alle arvertenze a pag. 2 e 48.

Catullus, Tibulles, Propertius. Ex ree. C. Lachmanni. Berolini, 1829,3 vol.

- Quae extant omnia cura Robinson Ellis, J. P. Postgate, J, S. Phillimore, 1911.

- Rec. et praefatus est L. Hueller, Lipsiae, Teubner.

Catcluds. Ex rec. C. Lachmanni, Ed. altera, Berol., 1861.

- Rec. L. Sehwabe, Gissae, 1866.

- Gedichte in ihrem geschichtl. Zusammenhange, übers. u. erläutert v. R. Westphal., 2 Ausg., Breslau, 1870.

- Rec. appar. crit., proleg., appendices add. R. Ellis, Oxford, 1878.

- Texte lat. av. comment. crit. et explicatif par Benoist et Thomas, et trad. frane. par Ed. Rostand, Paris, 1882.

- Herausg. u. erklärt von A. Riese, Leipzig, 1884.

- Rec. B. Schmidt., ed. maior, Lipsiae, 1887.

- Erklärt von 6. Friedrich, Leipzig, 1908. 

I.

IL POETA LIIINIO ('ALVO 


\section{AYVERTEN/A}

Diamo l' indicazione bibliografica delle principali opere contenenti i frammenti, citate di solito nel corso del lavoro toi soli nomi desli autori :

Aug. Weichert, Poptarmm Latihorum IIostii, Laerii, C. Licinii ralri, C. Ilrlrii Cimnar, C. Ialgii Ruti, Domitii Marsi vitae "t eremimum enliquine, Lipsiae, 1830, 1. $89-146$.

1. Mueller, Laevii Calci aliormm Carminum relipmiac, nella ediz.

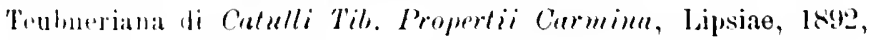
1. S.3-8.

le. liathens, Fragmentu l'netarnm Romanornm, Jipsiae, Teubner, r. $3000-3020$.

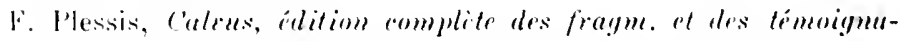

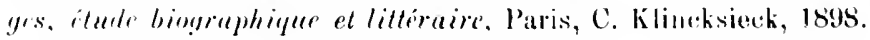

Manna livinio Calvo mella scelta fatta la

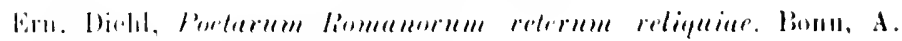

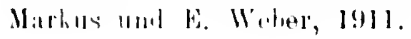


In uno di quei suoi brevi e taglienti epigrammi, che sono sprazzi di luce così viva sulla età Cesariana, Catullo riferisce un aneddoto su Licinio Calvo, sul suo Calvo, com'egli dice (mens Calcus). Hentre questi una volta snocciolava in una orazione tutte le colpe del famoso P. $V$ atinio, del quale era acerrimo nemico, uno della folla degli spettatori clie assistera al processo, levò in últo le mavi in segno di ammirazione (1), ed esclamù: ' di magni, sulopugium disertum!' (Carm. LIII, 5). Una espressione di carattere familiare e popolare, della quale è varia l'iuterpretazione; la forma comunemente adottata è salupittinm: ora altri vide nel saluputtirm qualche cosa come un 'libidinosetto', ed altri unia 'squillante trombetta', ed altri un 'giovanottino' ('); ma quale si sia l'interpre-

(1) Cosi efr. Cic. Acarl. 2,63 a Hortensins rehementer admirans, ut etiam mauns saepe tolleret».

(2) Secondo alcuni la prima parte appartiene alla ral. di salax, e la seconda è il putium di praeputium; cfr. Keller, Volksetym. 69. Il Keller stesso rammenta la spiegazione, che riconduce la parola a salıptta, salpicta, gr. $\sigma \alpha \lambda \pi \iota x \tau \gamma_{s}$, 'trombettiere'; ufr. anche Thielmann, Arch. lat. Lex, IV, $601 \mathrm{sgg.,}$ e Walcle, Etym. 
tazione clue si accetti "si propugni, questo pui ritenersi carto. che nellat parola deve essere racchiuso uno scherzo mu po' yosislanu e pleben: mal di quelle parole triviali lel volgo, drelle quali un persumagreio comsumato nelle lotte poteval piutosto surrilere che offemblersene: ed in questu catiso poi, osservava il P'atiu, il disertum faceva pilsiare il selluputtinm!

Mal un altra osiervazione qui cade a proposito. Seneca coni cita l'espressione, da mostrate che la parola sia all(o) viral neel lingualgaio, e che lel suo significato egli

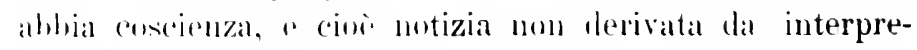

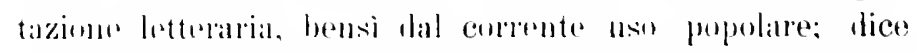

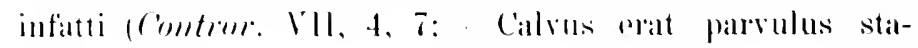

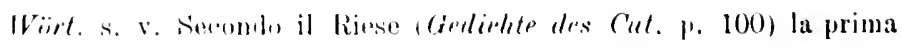

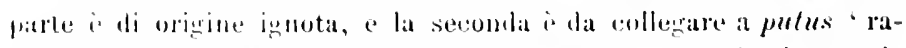

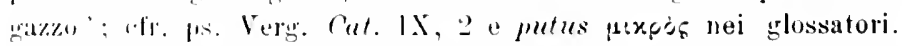

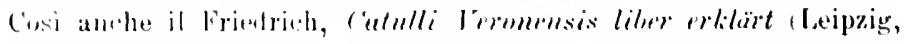

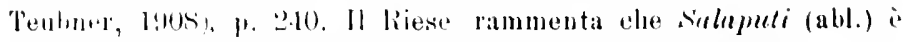
anche mone proprio, e rimanda a linecheler, lik. .lus. 37, 530.

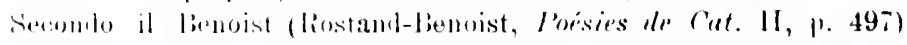

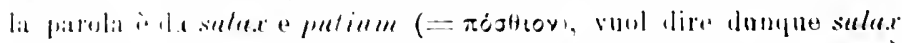

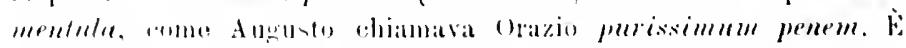

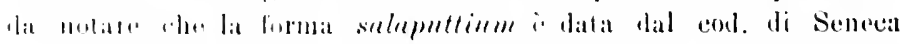

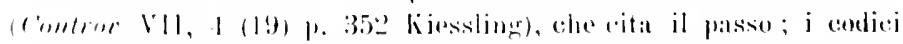

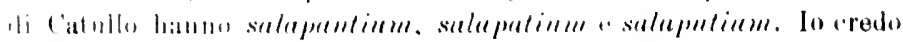

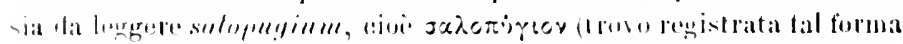

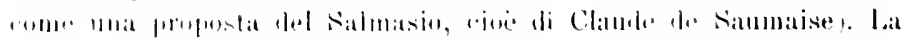

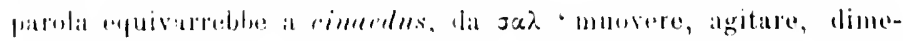

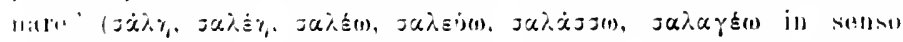

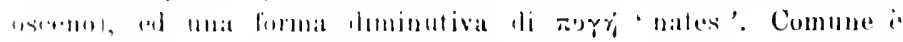

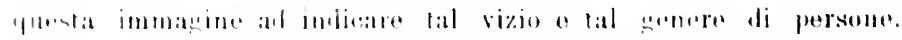

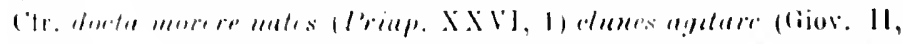

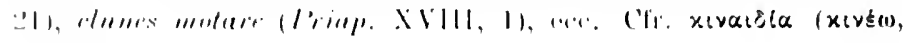
aiz(i) 
tura, propter quod et Catullus in hendecasyllabis rocat illum salaputtium (rosi il rorl.) disertum ».

Pure se la malizia principale chiusa nel motto ci sfugge, l'impressione che si ha dal pasiso di Seneca, ravicinato a quello di Catullo, is che Calvo fosse cosi chiamato perchè era un piccolo diaroletto eloquente, diavoletto, ho sostituito io: ma l'immargine che si cela nella misteriosa parola sarà stata oscena, di quelle di cui i volghi italici han fornito sempre gran copia.

Ton senza ragione dunque questo piccolo prodigio di spirito e di facondia era a Catullo carissimo: e la tradizione letteraria a lui strettamente lo lega. Ed anzitutto dal libretto di Catullo stesso risulta la prova di una grande intimità di affetto e di una grande affiniti di tendenze politiche e letterarie.

L'intimità si ravisa e nella doro vita sollazzevole, e nelle wioconde trovate di spirito e nelle loro sciagure. Calvo, in pagamento della sua opera di avvocato (1), aveva riceruto dal cliente sulla un dono singolare: nna racenlta di poeti. Ma erano pessimi poeti: ed egli, certo per amicherole scherzo, se ne libera. scaricandoli sopra l'innocente Catullo. E questi grli manda per risposta un grazioso componimento. Se eqli non l'amasse più rlegli occhi suni, l'odierebbe ora puanto l'odia Tatinio: ma poiche non può, se ne vendicherá col dargli il ricambio di almo simile florilegio di pessimi poeti, Cesio, Aquino, suffeno, e tutti gli altri veleni, tutti gli altri saccli incommoda! (carm. XIV).

Un altro carme di Catullo, il I, ci mostra i due con-

(1) È cosi da intenulere il Guod non" dispereumt tui lubores dell'epigramma XIV, 11. 
pagni in ma delle loro amicheroli gare di pousia. Avevamo trascorso la griomata tra il rino e gli ozii delle Muse. seambiandusi i loro versi e tentando ora un metro ora 111 altro. Quando Catullo tomo a easa. era eosi acceso dello spirito e delle facezie dellamieo, elıe nom potè prendere somn, e dibattendosi nel letto lesiderava la luce per tornare da Caloo. e stare con hui e parlarrali! Anche nell amicizia l’indole di Catullo è rjolenta e passionata.

Sella sciagura gimuge a Calvo il canto del sla preta. Quando infatti Calro perde la sua diletta Quintilia. Catullo wli manda un soavissimo carme (XCVI), che non ir di confurto. ma i pieno di mistien ineanto: la defunta Quintilia is quasi evocata dall' oltretomba: l'antico amore ririve nel cuore di Calro, e Quintilia si compiace di quell'amore che dura oltre le legrei del fato piu ehe non si dulga della sua morte immatura:

Si quicquam mutis gratum arseptume sepuleris

Accilere a nostro. Calve. dolure potest.

buom lesilerio veteres renovamus amores.

Atrue olim missas flemus amiritias.

Certe non tanto mors inmatura dolorist

Guintiliae, quantuns gaudet amore tuo.

$$
\dot{*}+
$$

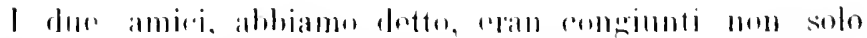

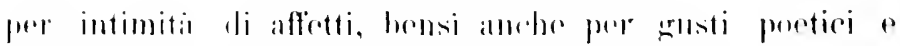

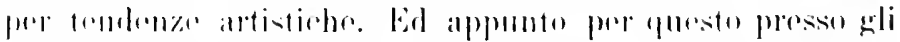

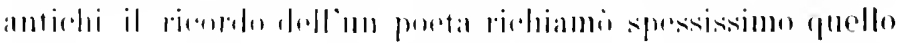

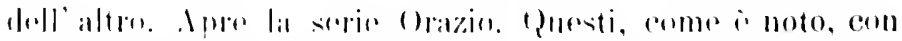

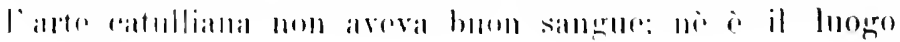


qui d'indagarne le ragioni (1); ed in un luogo famoso delle satire se la prende appunto con quegli scimmiotti, come egli dice, non buoni ad altro che a recitare Calvo e Catullo (Sat. I, 10, 19): "neque simius iste Nil praeter Calvom et doctus cantare Catullum ». Evidentemente v'erano ai tempi di Orazio grli appassionati dei due poeti. Na dell'atteggiamento sprezzante del Venosino può fare giustizia un uomo di finissimo gusto, e che Orazio stesso tenera nel massimo conto. Mecenate. Egli volendo dire una tenerezza al suo Orazio, ricorse ad un verso di imitazione catulliana. "Ni te viscerihus meis, Horati, Plus iam diligo » (2) gli scrisse: ed c̀ frase foggiata su quella di Catullo a Calro: " $\mathrm{Ni}$ te plus oculis meis amarem, Iocundissime Calve (3).

Insieme nomina i due poeti Properzio, auspicando a sè la fama quasi in confronto alla loro (II. 25 [III, 20], t):

Ista meis fiet notissima forma libellis,

Calve, tua renia, pace, Catulle, tua.

insieme li nomina Oridio, pregandoli di procedere insieme incontro a Tibullo, là negli Elisii (Amor. III, 9, 62):

Obvius huic venias hedera iuvenalia cinctus

Tempora cum Calvo. docte Catulle, tuo.

Plinio il giovane, volendo elogiare i versi di certo Pompeio Saturnino li paragona appunto a quelli di Catullo

(1) Cfr. il min scritto Orasine Catullo in Athenaeum, luglio 1915. Contemporaneamente fu pubblicato lo scritto Horace, Catullus and Tigellius di B. L. ITlman, in Classical I'hilology, Iuly 1915. p. 279-296, che muove da criterii affatto diversi.

(2) Svetonio. Vita Horatii.

(3) Carm. XIr, 1. 
e di Calvo (1): frecit ressus quules Catmllus ant Calr.ns; e ne determina il carattere angimmenuln che quegli intramezza, come Catullo e Calro, ai versi teneri e molli versi più duretti duriusculos yuosdam. Un amico ed elnrriatore di Plinio, Senzio Augurino, pur si propone quei due modelli, ma poi tosto li abhandona, gracchè Plinio vale per tutti:

Canto varmina versihus minutis

His olim quibus et meus Catullus

Et Calvus veteresque, sed quid ad mo:

l'nus Plinius est mihi priores... (2)

In una discussione critica, della quale c' informa (iellio, tra alcuni rotti Greci ed il retore Giuliano, quelli lomanlavamo se vi fosse tra i poeti romani chi per squisitezza - fluiditi di rersi fosse paraconabile an Anacromte ed agli altri poeti greei di tal genere, salvuche per poche cose Catullo e parimenti per poehe cose Calro (3). $\dot{E}$ dunque tutto un coro di critici antichi, che ravisavamn i medesimi caratteri nell'un preta e nellaaltro (4). Indaghia-

(1) Plinio, rpist. I, 16, 5 .

(2) Versi riportati da Plinio in una epistola $1 \mathrm{~V}, 27,4$.

(3) Ciellin XIX, 9, 7: "(Aracei plusculi Inlimmme rheterem) percontabantur quid de Anasreonte ceterisque id genus poetis sentiret of meguis nostrorum protarum tam fluentes arminum delicias ferisset? 'Misi Catullus' inquibut forte pauca of Calrus itidem palla:

(4) Anche Nonio Mareelle, 198, 11 li cita insieme per il comune use di remis al fomminile. Es como is naturale, anclie i commentalori al passo sopra ritato di Oratzo: Iortirione, anl Hor. Serme I,

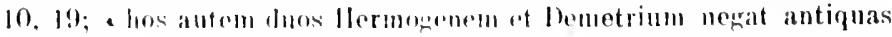

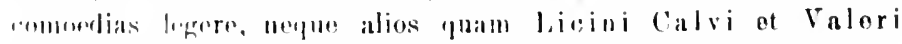
Calulli rersus merlulari o; Comm. Cruequii, a l. e. : a sl nihil 
moli questi earatteri, per quanto è dato a noi fare. sulla seorta delle antiche testimonianze e degli scarsi frammenti.

$$
\text { * } * \text { * }
$$

Un carattere comun a Catullo e Calvo riguarda non gia il genere di componimento o le tendenze poetiche, bensi la forma e la tecnica dell' arte. ¿̀ un carattere che è rilevato da Plinio il giovane, in una sua lettera, a proposito del poeta Pompeio Saturnino. Egli dice (Ep. I, 16, 5) ehe questi soleva a bella posta inserire tra i rersi mollcs e leres, aleuni alquanto duretti, durinsculos quostam, e eiò come facerano Catullo e Calvo. si trattera, oltrechè di partieolarità metriche, di espressioni antiquate o dotte, o di suono sgradito. Tali infatti ne furono rimproverate a Calvo, prosatore e poeta, dagli antichi; e at es. da Quintiliano (1, 6, 42) la forma collos, dat Carisio, (I, 59) ha forma tricliniarius per tricliniaris (1). Per Catullo basterà ram-

aliud doctus quam ad carmina Licini Calvi et Valeri Catulli decantanda ". Nota pure che Marziale fa seguire al un epigramma per Catullo uno per Calvo (XIV, 196 " Calvi de aquae frigidue usu, lez. incerta).

(1) Cfr. Weichert, Pnetarm lat. Reliquice, p. 123; L. Mueller, fr. 20; Plessis, n. 24. Il easo tel doppione triclimiaris e tricliniarius e pari a quello di iornlaris (Terenzio, Phom. 1, 2, 84) e iocularius (Terenzio, Andria IV, 4, 43). Delle tue forme altri sostenne la declinazione in -is esser nata per contrazione da quella in -ius, altri essere anteriori le forme in -aris, sulle quali la lingua posteriore avrethe coniato poi le former in -arius. Su questi aggettivi in -aris ed -arius veli Ritschl, De declinatione latima quadam reconditiore, p. 20 ; Benseler, the mominibus proprii et Latinis in -is fr', -ius et Graecis in -65. - $6 \mathrm{~V}$ pro -605, -60v terminatis, (Diss. inaug.) Lipsiae, 1870, p. 11. [ $=157]$. 
mentare mutriess (LIV, 18) per 'mammelle : e flexerunt artus (I,IV, 305) per 'si assisero'. ed altre simili.

$$
* * ;
$$

Eno dei piì frequenti attributi dati a Catullo è quello di dortus. Cousi lo chiama Ovidio, in quel passo famoso, degli Amores sopra citato. nel quale lo suppone insieme con Calvo asli Elisii, rum C'ulvo, dorte ratulle, tuo (III, 9. 62): cosi lo chiama liurdamo, rammentando la rappresentazione catulliana di Arianna abhandonata:

Sic cecinit pron te. doctus, Minoi. Catullus. Ingrati referens impia facta viri.

$$
\text { (ps. Tih. IlI, 6, 41) }
$$

Cosi anche i ehiamato Catullo piì volte da Marziale (1) e la qualehe grammatico (2); e lappellazione (hbe fortuna anche quando. molto probahilmente, quelli che l'adoperavano non arrano $u n$ chiaro eoncetto del suo simnifieato: o forse appunto per questo?

Come Catullo, o chiamato dortus anche Calvo, e da serittore molto autorevole, Properzio (II, 3., s.9-f1], 32, 891): IJare rtiam dorti ronfossa est pagima Calvi.

Catullo a calro sono dunque due poeti docti; ma comer i da intendere la dertrine attrihuita ai due poeti? lis statn spesse richiamato un pasiso dell orator cicero-

(1) Marmialn 1, lil, 1: Voromat doreti syllahas amat vatis; Vll,

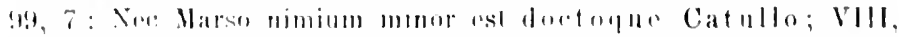

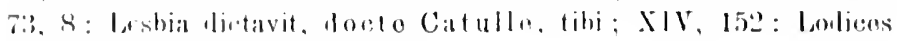
mittot dorti thi torra Catulli.

(2) Terenziano Manro. "2562: docti... Catulli. 
niano, nel quale si è voluto vedere ma ironia contro la nuora scuola poetica, la scuola appunto dei poetae docti (1). Cicerone parla in quel punto (s 161) di una differenza nella valutazione metrica dell's finale di parola tra gli antichi e i nuori poeti. Gli antichi gradivano le unioni, come omnibu princeps, dignu loroqne, elidendo innanzi a consonante l's di omnitus e di dignus; ed in tali unioni sentivano un dolce snono. una succitas. Inrece $i$ suoi contemporanei ri sentirano un suono sgradito, una offensio, e i poeti nuovi la evitavano: ea offensio.... quam nunc fugiunt poetre novi. Cicerone ̀̀ decisamente per gli antichi ed esprime in forma ironica la sua preferenza: se l'incolta consuetudine è capace di tanta dolcezza di suono, che cosa può aspettarsi dall'arte raffinata? Quod si indocta consuetudo tam est artifex suavitatis, quid al ipsa tandem arte et dortrina postulari putamus? (\$ 161). Indocta, doctrina: l'allusione ironica è evidente. Na dovremmo cercare solo qui il carattere dei poctae dorti? In un senso diverso di musicalità poetica e di armonia delle parole? in relita si trat-

(1). V. su tutta la questione dei poeti vecirepor il buon volume di Adolfo Gandiglio, Cantores Euphorionis, Bologna, Nic. Zaniehelli, 1304; e sul significato di docti detto di poeti F. Barta, Ucber die auf dic Dichthunst be iuglichen Ausdrïcke bei den röm. Dichtern. I. Linz, $1 \$ 89$ (progr.) 1). 26. Giustamente il Lejay (les Satires dithorace, Paris, Hachette, 1911, 1. 235), a proposito clel docti sumus (Sat. IX, 7) detto dal secuatore oraziano, annota: - Le mot designe la culturo littéraire et souvent le talent poietique: Sat. X, 87; Epist. II. 4, 56, 117 ; Oviul. A. A. III, 5うl etc. e rimanda alla dissertazione sopra citata del Barta. All'ispirazione e all' arte riferisce l'appellazione doctus Calnllus l'Alhini, in Atene e Roma, XIV (1911), p. $356 \mathrm{sg}$. 
terebhe di hen poeo. Che i poetae uori rolessero far mostra di ma delicatezan magriore, e di piò squisito gusto. e chiamassern rozzi ed incolti gli altri, e che Cicerome esprimendo con fine ironia il loro pensiero chiamasse fortrina la loro squisita ricercatezza ed indocti gli altri. si comprende e si spiegri. Ma questo piccolo particolare non ci soddisfa più, quando si tratta di spiegare il vocabolu dortus attribuito a due poeti di amorc quasi come carattere peculiare della loro arte. ed attribuito da persone che non si prenceuparano più nè di s tinale nè di altre cotali particolarita metriche, essendo ormai diventato di universale dominio il rinuovato zusto poetico. La dortrina mon deve liguardare un carattere esteriore e formale dei due poeti, bensi piuttosto un carattere intrinseco della loro arte.

Neppur sarebbe wiusto intendere di veral e propria 'dottrina: nel senso errave della parola. per moda che Catullı, l' slegantissimus poetarmm secondu Gellio (11, 20, 6), sarebbe stato considerato un ben gravosin e pesante poeta. La rerita è che dortus va inteso en molta discrezione: altro i che si chiami durtus Imo storice " In erudito ed alto i che si chiami /orlı ma fanciulla : e Catullo stesso arri volute, significare due eose diverse quando chiamava dorti i libri di Cornelio Sepore (1. 7) (1) e quando chia-

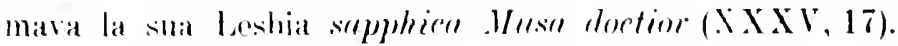

l)a tal distinzinne hisugual, credo, partire (2). Questi

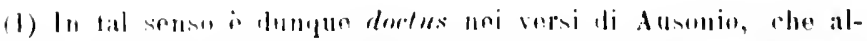
Iurlumo appunto a Cormolin Sopote: Inermi... mer doctum mimus

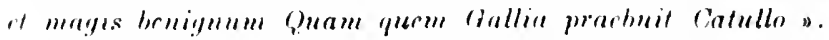

(2) Morfus i anche "perito nolla preprie arte', quindi prosso (1razio. Epest. 11, 1, s2 doctus Rosrius. Dorti sono ancho i bilo- 
poeti non celebravano ragazze ignoranti del popolo, ma ragazze che si piccavano di letteratura, e leggevano i poeti greci d'amore $\mathrm{e}$ se ne deliziavauo. I poeti (sempre adulatori con le domne!) chiamavano dotte le loro Juse. Così, come abbiamo visto, chiama Catullo Lesbia: così chiama sulpicia l'ignoto poeta del libro IV Tibulliano (1), così ripetutamente chiama la sua fanciulla Properzio (2). E l' esempio di Properzio è istruttivo, gialche il poeta stesso ci dice in che cosa consistera lat dortrina di Cinzia $(\mathrm{I}, 2,27-30)$ :

Cun tibi praesentim Phoebus sua carmina donet Aoniamque libens Calliopea lyram, Unica nee desit iocundis gratia verbis,

Omnia quaeque Venus quaeque Minerva probat.

Cinzia dunque era dorta, perchè ficera versi fanche questo, oltre le colpe di amore !). e sumuava la lira, ed

sufi; quindi presso Grazio stesso, Sat. II, 4, 3 doctum Platona. Ed anche Urazio dhiama dutto Pacuvio, Epist. II, 1, 50 Pacnrii docti famam senis, ed io credo a un dipresso nel senso in cui noi diciamo 'dotto' Catullo, giacebè sappiamo the Pacuvio trovava * duriora paulam et acerbiora i carni del giovane Accio (Gellio, XIIl, 2, 2), il che mi pare indichi studiu di squisitezza $\theta$ di eleganza; e così mi sembra che interpreti anche quintiliano, X, 1,97: "Virium tamen Altio plas tribuitur: l'arucium rideri ductiorem, qui esse docti affectant, volnnt». E vera duttrina, cioi sapienza e senno, indicheranno il Simonides doctus sapiensque di Cicerone, (I) Nat. Deor. I, 22), e il Maecenas docte di Orazio (Epist. I, 19, 1). Cfr. Weichert, Poet. Lat. Rel., f. 121-2, nota 27.

(1) Ps. Tib. IV, 6, 2: Quos tibi dat tenera docta puella manu.

(2) Prop. I, 7, 11: We laudent ducta solum placuisse puellae; $11,11,6=111,2,6$ : Nee dicet: cinis hic docta puella fuit: III, 13, 11: Ve iuret in gremio doctae legise puellae. 
area grazia nel parlare ed avera le doti di Venere e di Minerva. Dortus ì da intendere dunque in un senso mondano (1). Nei salotti delle eleganti dame romane la doctrima non era l'erudizione, ma era la poesia amorosa e la musica ed ogni altra arte gaia e leggiadra. A quella dottrina presiedevano non le Juse severe, ma le Grazie. E fu chicmatil dotta Atene, madre delle arti gentili (2), e dotta la cetra di Apollo (3), e dotte le fronti dei poeti (4), e dotto giovane il pastore Teocrito (5), e dotti i poeti d'amore (6).

$$
\text { * * * * }
$$

Un altra questione ar qui si presenta. Come sarà da interpretare l'ovidiano: "pur fuit erigni similisque lirentiul Calli: (Tirist. 11, 431)?

E stato mesiso in rappentn quell exigui Caldi eon la

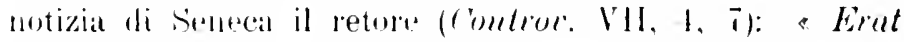
(culews) perrolus stutura ».

Ha mon sarri da intendere invece di una qualiti del suo ingerno protion, quatsi: 'sottile. tonue, delicato'? Qualehe cosa di moltu similu veliamo alfermato di Cit-

(1) A mu dipresso conne cullus: (1vil. Am. III, 15: Discentur unureri. anlte 'Jibulle, tui.

(2) Cialul. ps. Verg. XV, : : limma... duetis certaret Athenis.

(a) I'rop. IH, 34, 7!: Tale facis cammen, docta tostudine puale Cyuthius inpositis tomprerat artioulis.

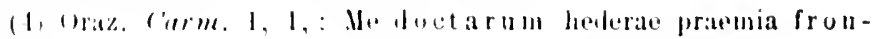
tiulli.

(5) ratul. ps. Verer. IS, 20: dest us iuvenis.

(b) Thb. 1, 4, (j): Mirridas, puri, doctos ot amate poetas. 
tullo da Marziale (X, 103, 5): Nec sua plus debet temui Verona Catullo. Quel che Marziale intendesse nel chiamare temuis Catullo, si può argomentare da altro passo di Marziale stesso, ove Catullo è chiamato tener (XII, 44, 5), Lesbia cum tenero te posset amare Catullo, Te post $\mathrm{Na}$ sonem blanda Corinna sequi. Nelle poesie di genere amoroso dunque, nei piccoli epigrammi di carattere sentimentale, si riconosceva la grazia e la delicatezza Catulliana, ed a ciò vuol riferirsi Marziale col chiamare temuis o tener il poeta Veronese.

A me pare che l'exiguus attribuito da Ovidio a Calvo sia proprio il fratello germano di quel temis. Si tratteri di quelle fluentes carminum deliciae, che, a proposito appunto di Catullo e Calro, troviamo menzionate dia Gellio (XIX, 9, 7): di quei rersi insomma dell' uno e dell' altro, dei quali Plinio dicera (Epist. I, 16, 5): quantum illis leporis, dulcedinis, amuritudinis, amoris!, lodi che sono riferite bensì da Plinio ai versi di Pompeio Saturnino, ma che ridondano, come risulta da Plinio stesso, ai suoi modelli, Catullo e Calvo.

Tenui e delicati poeti dnnque, quando rolerano; mat quali spiriti e quale impetuosa forza aggressiva assumevano talvolta questi gentili poeti d'amore!

Ben lo riconosce Marziale stesso, che adopera l'espressione (XII, 83, 4): Quantum nee duo dicerent Catulli, quasi a segnare il termine ultimo dell'asprezza nell' invettiva; e Quintiliano rammenta l'acerbitas dei giambi di Catullo, di Bibaculo e di Orazio (Inst. X, 1, 96).

Anche in questo, lalvo fu affine a Catullo di tendenze e di spiriti poetici. Lygli portava nei giambi veementi lia medesima irrueuza, che il reudera terribile arversario 
nei dibattimenti del foro (1): e cel dice Seneca retore (Contror. III, 19 [I'II, 4, 7]): carmina quoque eins, quamris iorosa sint, plena smot ingentis nnimi.

$$
* *
$$

Verliam, ora in quali occasioni e contro chi ebbero a prorompere le violenze agoressive dell' un poeta e dell’altro. Un medesimo capitolo di Suetonio ci informa dei lon assalti contro Cesare o alcuni principali nomini del partito di Cesalre (2). Egrli rammenta che C. Calvo scrisse contro Cesare epigrammi lenigratorii, famosa epigrammula, ma che fini poi per desilerare egrli stesso la riconciliazione, e mettere in mezzo wli amici per avviarne 1. pratiche: a Césiure, con uno dei snoi tratti di pronta lecosinne, prel troncaure gl indugi, scrisse per primo a Calvin.

P'er Catullo le cose procenlettero parimenti spedite. Nel

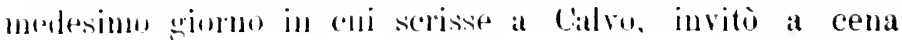

(1) Calvo ancle nelle orazioni sapeva essere, socolldo $i$ casi. com-itato e solenne, " line enl arguto. Crr. Senecil, I. r. \& violentus accusator et eoncitatus fuit "; Quintiliano $X, 1,115$ a sel est et

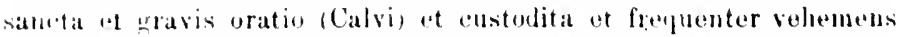
yuofue ; Cire. Lirut. 81: "Atticum se Calvus dici oratorem rolebat ; inde erat ista rxilitas, quam illa de industria consequebatur : Apuleio, Apol.95: "in illa coratione Aviti nec Cato gravitaltem repuirat... nee Calvas argutiat .

(2) Suet. Cars. 73: C. Caloo post fammsa epigrammata de re-

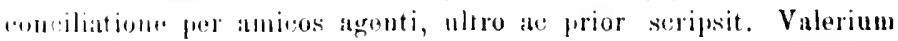
Catullum, a quo sibi vessiculis de Ilammora perpetua stigmata in-

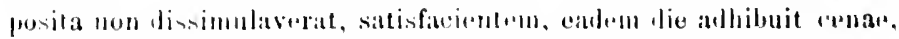

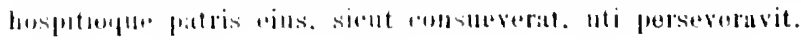


Catullo, i cui epigrammi contro Mamura averano bollato anche hui, come Cesare stesso avera riconosciuto, di un marchio pereme. Questo ci riferisce suetonio; ed un’attestazione del pari antorerole ha Tacito. Nel libro IV degli Amuli (cap. XXXlV) ardi racconta del processo intentato dali satelliti di Séano a Cremuzio Cordo, pes avere negli Ammuli chiamato C. Cassio l'ultimo dei Romani Cremuzio Corlo si difese nobilmente dinanzi al Senato, rierucando l'antica libertá di pensirro ed adducendone gloriosi esempii: e tra sti altri appunto puello di Catullo, contro cui nulla tentio nai Cesare: "cramina Biburmli et c'utulli refertn antumelies Cuesormm leynutur; sed ipse dirus Inlins, ipse dions Amynstus et tulere ista et reliquere » (1). Se is ha stare all informazione, sopra riportata, di Suetonio, uno stessis grionos seguo la pace tra Cesalre da una parte, Caltullo e Citvo dall'altra. I due amici procederano di pieno aceordo anche nella loro azome di vita pubblica, e nelle manifestazioni delle toro simpatic el antipatie politiche.

Degli epigrammi di Catullo contro .ramurra e contro Cesare, delle sue accuse sanguinose contro quest' ultimo, cui egli fa risalire la responsabiliti ded lusso sfrenato di Mammar, dei rizii immomdi, delle depredazioni e spoliazioni di proviucie, uon is qui il lungo di partare (2) : sol

(1) Cfr. G. II. Bernstein, Tersus lndicri in Romanorum Capsares priores olim compositi, Halis Saxonum, MDCCCX, p. XIX $\mathrm{sg}$.

(2) Carmi XXIX, LIV, LVII, XCIII. Mamurra, che era di Formio, is anche if Formiamus dei carmi XLI e XLIII. Secondo una

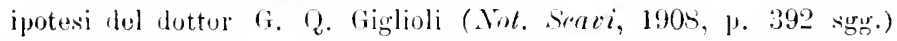
Manura appartenera alla Gens fitmria : v. di cii E. Pais, in Atti R. Acr. Areh. Lefter e li. A. Niapuli, 1913, p. :3-4. 
vorliamo rammentare che nei rersi stessi di Catullo, e propriamonte nel came XI, è la prova dell arventa riconciliazone, griaceho ivi di cesalre si parla non più chiamambolo irmicamente Rommlns, imperator micus (1), hemsi chiamamdolo con encomin solemes al trionfature dei Britami, il gran ('esare (X1, 10): "Caesaris risens momelmente mugyui 》(2).

l)i cii che Cillo serisse contro Cesalle rimane qualche

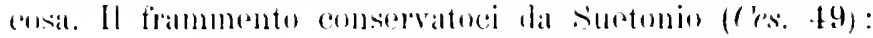

fi. I. Bithyuia quidyuid

lit predirator Caesaris umeguam habuit.

pui appartemere all uno dei famosel epigrammala, o anche puis essere an allusione posta invilentalmente in altor compunimento, ma is ma torribile allusione. A si-

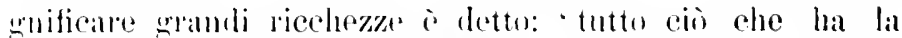

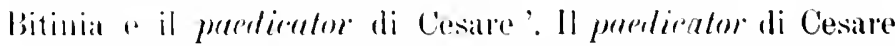

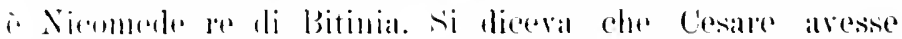

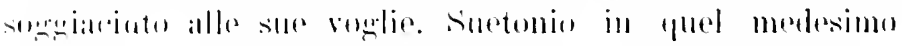

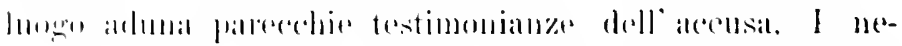

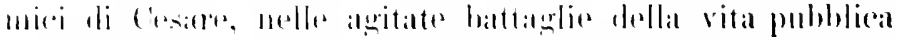

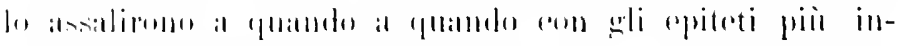

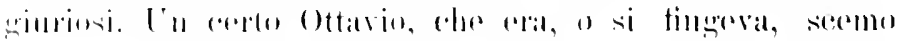

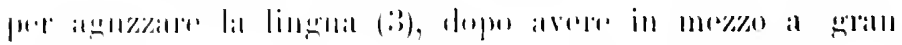

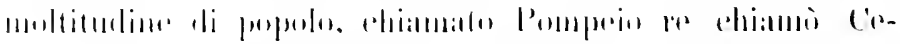

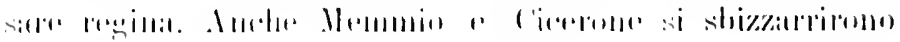

11) Carmi XXIX, IIS.

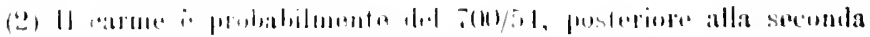

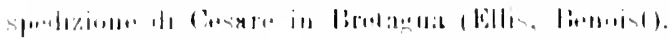

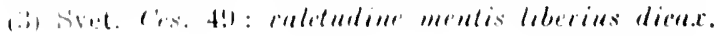


atrocemente nell' accusa (1). Ed i soldati di Cesare durante il trionfo gallico cantarano (2):

Gallias Caesar subegit. Nicomedes Caesarem, Ecce Caesar nunc triumphat, qui subegit Gallias, Nicomedes non trimmphat qui sulegit Caesarem.

Ed anclse Catullo, nol carme XXIX chiama Cesare ripetutamente Cinaede Rommle, e ripetutamente inpulicus et rorar et helluo.

Tutte queste accuse sono sospette per la loro stessa ferocia el insistenza. La passione politica spiega molte cuse. E la fonte mincipale delle accuse is molto impura, Memmio! (:3).

$$
* *
$$

Se gli strali di Catullo e di Calro si appuntarono contro Cesare, non rispamianomo però Pompeio. Il carme XXIX di Catullo is hensì terribile contro Cesare, che il poeta replicatamente chiama in cansa con la ingimiosa apostrofe: Cimuede Rommle, haee ridebis et feres? (v. 5 e 9) ; ma coinvolge nella respensabilita anche il suncero, cioè Pompeio, a rammenta la preda pontica disisipata da Mammral,

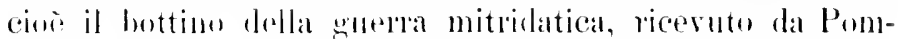
peio, (v. 1s) e tinisce con l'esclamare: " a tal titolo

(1) Suetonio, iri.

(2) Cfr. Stampini Fttore, Aleune osservaiomi sui cormi trionfali mmani, Torino, F. Loesoher, 1898 (likista di Filologia XX 1 I, fasc. 11), p. 9 e sgg. dell'estratto.

(3) Cfr. Munro, ('ritic. and Elmeid. p. 87 e seg., Benvist, Op.c. II, p. 443. 
dumpque voi, subero e renero, i più portenti cittadini di Rimma, arrete mamblatu tuttu a rurina "?

Eone nomine, urbis o putissimei

Socer yenerglte, perdidistis ommiat:

(v. $23-24)$.

Piò atroce contro Pompeion, benchis torechi la rita privatat, ir l' repigrammat CXIII di Catullo:

Consule Pompeio primum duo, Cinna, soletant

Mucillam: lacto consule unne irerum

Manserunt duo, sed revernut milia in unum

singula. Ferundum semen adnlterio.

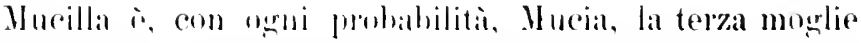
li Pompró, da lui poi ripuliata pre le sure infedelti nel b2 a. C. Il diminutivo mon fil meraviglia in Catullo, che

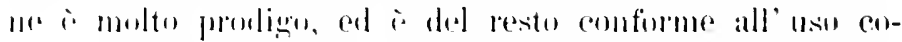
mune latimn, quando si tratti li tali femmine (1). 'T'ra i dur amanti della disonesta grimsane, ai quali allude l’e-

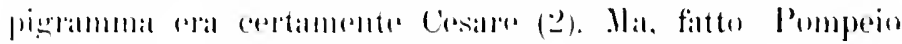

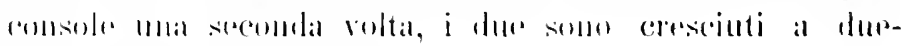
mila! Samgaimusu epigramma.

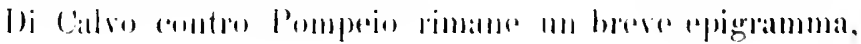

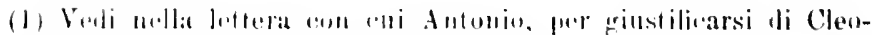

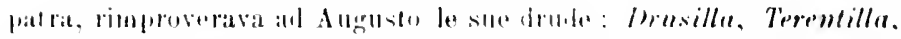

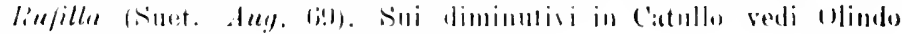

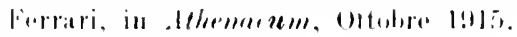

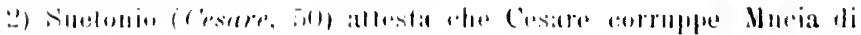
l'ympriv. 
nel quale, sotto mua forma un po’ uscura, sou contemute duc gravi accuse (1):

fr. II. Magnus, quem metuunt omnes, digito caput uno Scalpit. Quid eredas hune sibi velle: virum.

Per comprendere la doppia amarissima punta dell`epigramma, hisogna rammentare certi particolari dei costumi, o per meglio dire, dei pettegolezzi romani dell' epoca.

Il grattarsi il capo con un solo dito era dai malignii interpretato come tendenza alla vita effeminata, e quindi come atteggiamento da cinerlo. A Pompeio fu fatta appunto questa accusa. Ammiano Marcellino riferisce che gl' iniqui detrattori, nulla trorando a ridire su Pompeio, fecero notare come egrli aresse l'ahiturline di grattarsi il capo con un sol dito, e come con una benda candida egli avesse fasciato una ferita alla gamba: indizio l'una cosa di abitudini dissolute, l’altra di aspirazioni a resio dominio. Ammiano riustamente la per tanto petulante nequizia commosse parole di sulegno (2). Per la seconda

(1) Conservato presso Sthol. Lucani VII, 726, ov'è però attribuito a Marziale. Ma efr. Sen. Controv. III, 19: « et corminu quoque (Calvi), quamris iocosa sint, plena sunt ingentis animi. licit de Pompeio: digito caput uno " ecr. I. anche Seneca, Controv. V, 30; e Ammiano Marcellino XVII, 11.

(2) Ammiano Narcellino XVI1. 11, 4: * Nec non etiam in Pompreium obtrectatores inique multa serutantes, cum nihil unde vituperari deberet, inveniretur, duo haec observaverunt ludibriosa of irrita: quod genuino quodam more caput digito uno sealpebat: quodque aliquandiu tegendi ulceris causa deformis fasciola eandida crus colligabat: quorum alterum factitare ut dissolutum, alterum ut novarum rerum cupidum arlserehant : nibil interesse oblatrantes argumento subfrigido quam partem eorporis redimiret regiae maie- 
accusa le parole di Ammiann diludume ad nun seipito motto di Faronio, che rinfaceiando un gromo a Pompein la fascia hanca della sua ferita: " Som impurta, disse, in qual parte del corpo si porti il dialema! (1).

Perfino Cicerone, in una lettera ad Atticu, accenna a quella fascia, e ri trova si a ridice! (2).

E rediamo ora dell altra accusa. Calon la tolse da mal turpissima invettiva di Clodio, fatta innanzi al un hranco di smoi saltelliti.

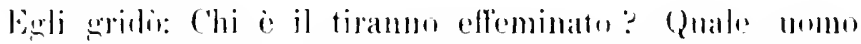
erea l'nomo? Chi con un sol ditn si gratta il capo? E quelli in com, compatti, a qram ruce: Pompein! (:i) Seneca parle, riferendosi appunto all accusa contemuta mell'epieramma di Caloo, par ehe roglia contrapponer la storia lel capitano vincitore per terrat e per mare: : In

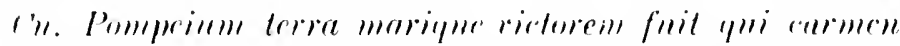

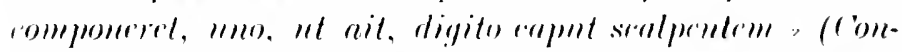
tror. V, 30). Questo melesimo atto del grattalri la testa

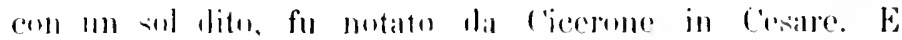

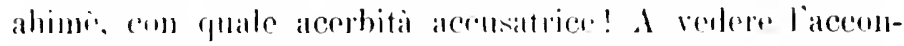

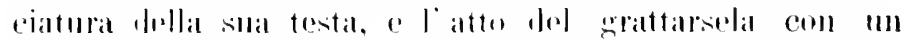

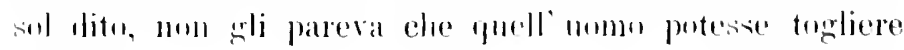

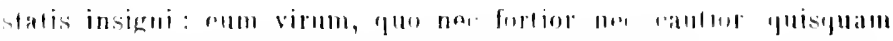

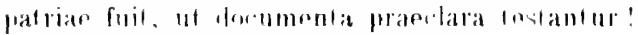

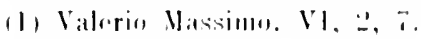

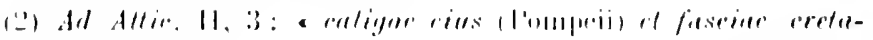

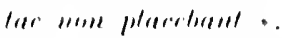

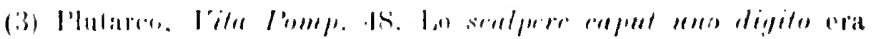

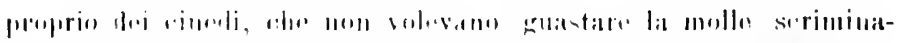

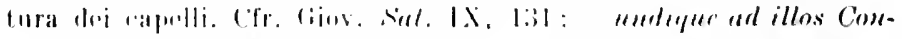

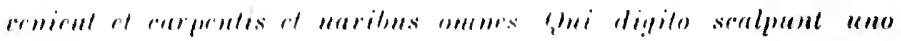
'alulul". 
Ia liberti ai Romani! (1). È questo appunto quel che viene a sinnifieare l'epigramma di Calvo contro Pompeio:

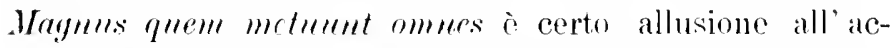
cusa che Pompeio aspirasse a dominio regio; ma non vi era ragione di temerlo, siaccho Pompeio era un cinedo!

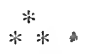

Anche contro un altro favorito di Crsare, il sardo Ermogene Tigellin, invei la Mnsa reemente li Calvo. Ci rimanc, conservatoci dallo scoliaste oraziano, un verso solo:

fr. III. Sardi Tigelli putidum caput venit ()

Cicerone in ma epistola (:3) allule a questo componimento contro Ermogene, e lo chiama Ipponattor preromio,

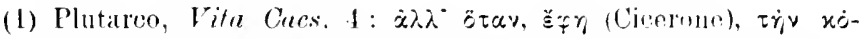

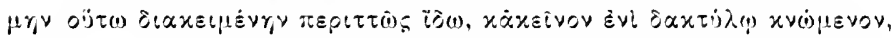

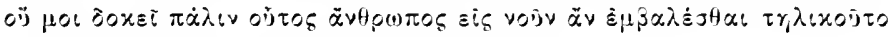

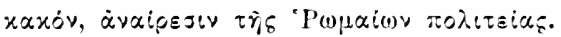

(2) Porfirione at llorat. siat. 1, 3, 1; "At Licinius Calvus de eodem Hermogene loruens. Sardi Tirelli ece.' v. E un senario riambico scazonte.

(3) Fam. VI1, 24: "enmune addistum ian tum puto esse Calvi Licinii hilponateo praceonio

Il eènit lel verso di Calvo ì illustrato da puel che Civerone stesso dice rlei siardi (Fum. VIl, 20 in f.) "hobrs strolos romulos ». - Questo Emogene cantons samo, a mominato la Grazio in due passi (sut. I, 2, 3; 1. 3, 3; mat non e rla confomelere ent l'altro Ermogene, anche cantore, che Hrazio nomina come vivente (Sat. I, 3, 129; 4, 72; 9, 25); 10, is e 90) e che era forse licrlio del primo. Che il primo fosise andev di Cesare risulta la Orazio, sut. 1, 3,5 (per amicitiom patris atupe suram, cioce di Cesare e di Ottaviano), e la Cicerone (Ftm. VII, 2) 1): "nom tam ab ullis quam hor tempore observm a familiarissimis Cacsaris, practer istum » (ciò Tigellio). 
certo a cagrione della momlacita e violenza agraressiva, per la quale exli paragoma Calvo al terribile Ipponatte. Il pulirlum copul di questo componimento dovè arer fortuna, se di essu troviamo una doppia riproduzione nei Cutalerto preurdovergiliani (1). Ma che cosa simuifica putidum rapul? Lo Ullman (:) interpretat dettu dello stile, pieno di alfettazione, e nota che Ciceme ( (), 27. Brut. 284) appunto in tal senso applica la parola arrli oratori asiatici, e che nella lettera di Cicepne (Fomm. VII, 24) nella quale i allusione all epicramma di Calro. Tigellio è chiamato satraro, che è la traduzione wreea di putirlus nel senso di 'affettato', sicche Cicerone stesso pare avere interpetato il putilum di Calro in tal senso. Teramente yuando Cierrone chiama salaro Tizellio, non pensa certo agli scritti, bensì al modo dr comportarsi: "rommosti

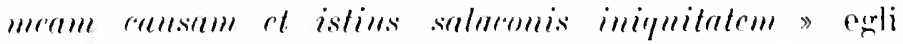
dice, e rmol simnificare: "li cotesto spacene, di cotesto ranesio (3). Ed is anzi da cschdore che eyli interpretasse il molielum di Calvo. come detto dello stole asiatico: egrli interpretio invece "schifisn'. come mi pare che ri-

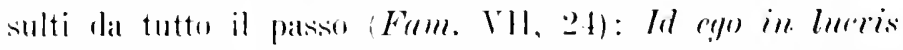

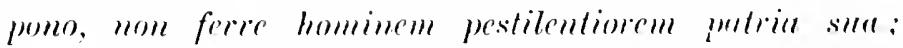

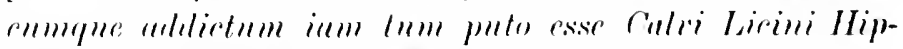

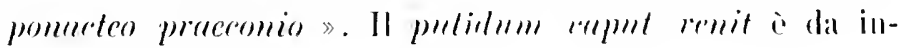

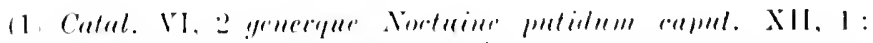

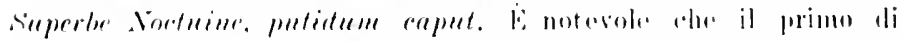

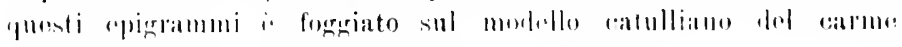
$\mathrm{XXX1X}$ " w riproluse il versu 25.

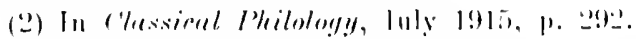

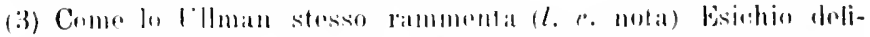

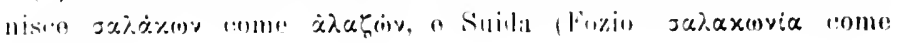

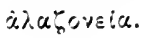


terpretare nel senso che Tigrellio, bencho ispiri ripugnanza fisica, si venda come cincdo: il pulirlus i detto molto spesso in materia erotica (1).

$$
* *
$$

Altro bersaglio delle satiriche inrettive di Calro fu Manio Curio, familiare di Antonio, di cui fa menzione Cicerone nella I Filippica (cap. 5) (2). Euli fu hiscazziere famoso ai suoi tempi, e di sua trista fama fil fede oltre Cicerone, Asconio Pediano (3), il quale aggiunge che fu pur condamnato. L' unico verso rimastoci del carme falecio che contro lui scagliò Calvo, accemma appunto a questo vizio di Curio. Esso ci è stato conservato appunto da Asconio nella forma: Et ealus Curims pereruditus. Calus non ha senso.

Il Baehrens e il Plessis videro in esso ma facile colruzione da ealx, il rettone nel wiunen dei dali (t):

(1) Cfr. Catullo XCVIII. 1 putide Vett; XIsll, 11 moceha putide (ripetuto nei vv. 12, 19 e 20); Orazio, eporl. VIll. 1 lonyo lutilum te saeculo. Nell' identico significato è putidum caput in ps. Verg. Catal. V1, 2; XII. 1.

(2) $\$ 13$ e 14; "... ut ei (.H. Antonio) cum Curio consessone codemque conlusore facillime possit conrenire... puto ne Curium quidem esse crudelem, qui periculum fortunae cottidic facit".

(3) Ad Cic, orat. in toga candida, p. 95 ediz. Orelli; 84 A Kiessling et R. Schoell : 1. 72 Stangl, Cic. Orat. Selnol, : "C'mius hic notissimus fuit aleator, damnatusque posten est : in hune rst hendecasyllabus Calri eleyans: Et talos Curius pererulitus s. Cirea la lezione del verso verli nel tesio.

(4) Baehrens. Fragm. poet. Hom. 1885. 1' 320; Piessis. Cutvus, p. 2. Il Plessis cita due frammenti li Lucilio (XIV, v. 10 e 11 ed. Müll. ma nel primo, invocato anclıe dal Baehrens, è dubbio 
l'alilio mimerpse di Ascomin ba talus: il Meichert, il

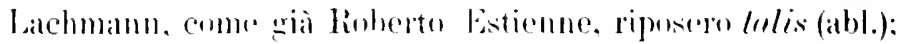
Léciann Miiller lalos (1), che noi reputiamu la piis prohabile lezime.

Pomiamo dongue il frammento nella forma:

fr. IV. Et talos Curius perernditus.

$$
* *
$$

Son sforki pretici, ma hen piin roventi parole scaglio Callo contru moltat ereatura di Cesale, Vatinio (2). Questi era awonte di cesare a limma " la lui protettu nella vita puhblicat wh ela statn promesiso it emsislato, ed exti se ne teneva siemo e per esin gimara, o, come

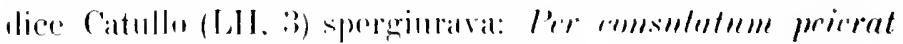

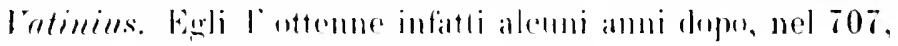
quando forse Catullo era wia mortu (in).

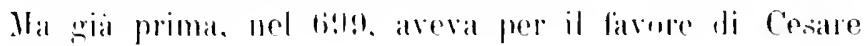

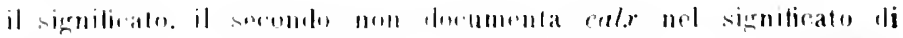

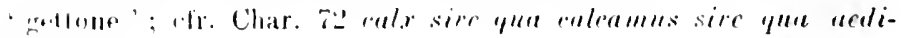
fir.amms.

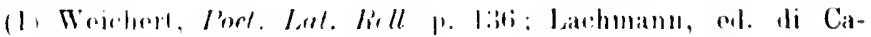

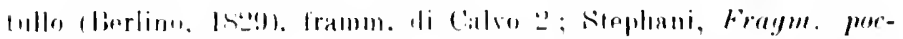

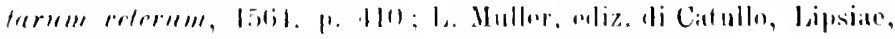

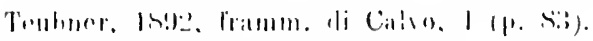

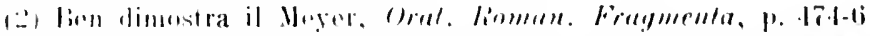

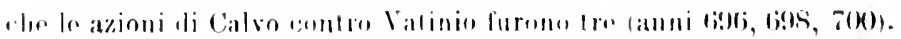

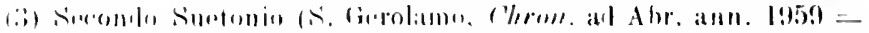

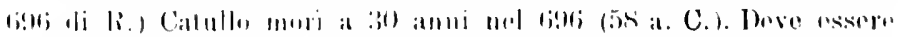

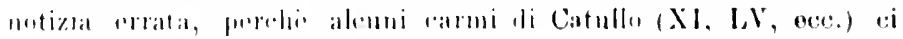

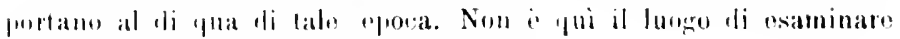
lo varie ipoteni fatte su tale argomento. 
ottenuto la pretura contro Catone: e nel luglio rel 700 accusito con veemenza da Calro, fu assolto appunto per la protezione di Cesare. Questa orazione di Calvo fu famosa; e del suo impeto aggressiro fal attestazione Seneca il retore. Mentre $u n$ ascoltatore di Calro gli sentiva esporre tutti i Tatiniane rrimina, usci stupefatto in quella esclamazione ammirativa: Di magni, salopmyimm divertum! che ci è stata conservata dal noto epigramma di Catullo (LIII) (1). Tatinio ne concepì un odio mortale contro Calro; siccho quando Catullo volle scherzale con l'amico per un brutto tiro che questi gli areva riunato, minaccio odiarlo di ollo latiniano (ram. XIV, 3). Vatinio era rel resto, per quallto sembra, ben tristo soguretto: e cicerme lo chiama: "odimme puhliemm popnli, sonatus, mimersorum rnstiranormm (In Fat. 16, 39) : siccho nom aveva forse torto Catullo, quando la sola posibilita (2) del con-

11) Vatinia stesso, riferisce senecd (Contror. IlI, 19: prorumpe disperato: Ma se costui è elmuente, è una ragione per conilannarmi ? Seneca stesso asserisce (ivi) : "violentus acusator et eoncitatus fuit. Solebat praeterea excedere subsellia sila ft impetu elatus usque in alversariorum partes transeurrere s. Lo molteplici testimonianze circa l'eloyuenza concitata el arcouta insieme di Calvo vedi presso Meyer, Orator. fom. fragm. 2 1. 47.4-4il;

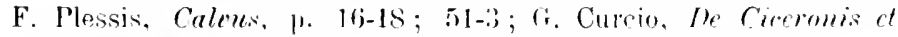
Calei reliquorumque Atticornum arte direndi, 1s!99, 1. Tt.

(2) Tale è eviınntemento il signitioato dell'epigramma Catulliano, non già che Vatinio aresse già ottenuto il consolato. Se questo fosse stato, il poefa arrebhe certo espresso con altra frase l'asereizio del potere consolare. Tatinio per confermare $i$ sun siuramenti soleva lire: "Te lo siuro, per quanlo è vero rb" in sarn console ». E cosi anduistara cremito ai suoj speryiuri, che somo rammentati anche da Cicerone (In Tat. I, 3): incomstamtiam, eum levitate tum etiam periurio inplieatam". 
solatn di Vatinio poncra come uno dei segrni di ultima decadenza politica, dopm il quale dovera essergli ormai srato il morive (rarm. III):

(Quid est, Catulle, quid moraris emori?

Sella in curuli struma Nonius solet,

Per consulatum peierat Vatinius:

Souil est, Catulle? quid moraris emori?

Come si vede, se Vatinio non fu bersarlio degli strali poetici di Calro, ma solo degli aratorii, a quelli penso l’amico Catullu.

\section{$* * *$}

Come pueta d'amore Calso a mella sorie degli antori di cami licenziosi o lascivi. (2uella serie $i$ ben luma in ma lettera di Plino (Epist. V. 3). mella quale cerea con esmpii illustri di scusan si per cotali debolezze. Eirli, si, licomosec, di far talvolta ressiculos parmm sereros; riconosee altre picenle marachelle: ma se ne ginstifiea con III motto: homon stem! [ Del resto ongli is in huoma comparnia: in eompannia anzi di tali, dei quali olode imitare nom le cose serie soltanto, ma amche le srocose. E qui

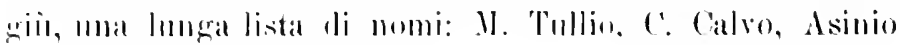
Polliome. .1. Messalla, erec.

Alla lista manca Catullo. conmer maneano T'ibullo Pro-

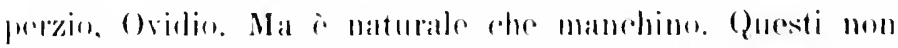

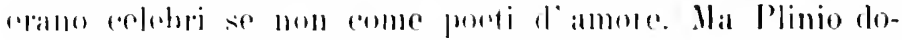

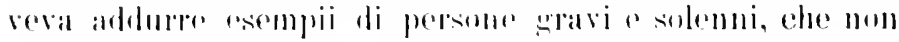

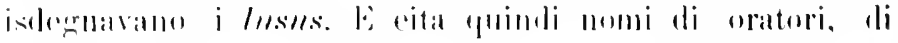

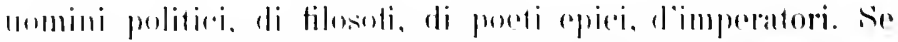

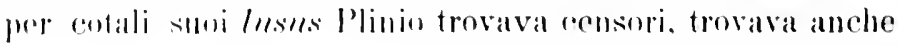


chi lo esaltava: anzi un giovane poeta, Senzio Augurino, scelse ad argomento di un proprio carme proprio questo, la degnazione di Plinio, che marmlt ressiculos foro relicto! Figurarsi la gioia di Plinio! Egli ne riporta un passo, e da esso nil pare risulti che i versus minnti attribuiti a Catullo ed a Calvo debbano intendersi proprio i rersionli parmm severi, le tenui poesie d'amore (1).

A questi carmi lascivi di Calvo accennano anche Properzio ed Ovilio, i quali vi fanno ricorso per il medesimo intento di Plinio: cercare autoriti per iscusare sò stessi.

Ha nei loro passi v'è qualche notizia più determinata di una semplice menzione. Properzio in un lungo carme

(1) Plinio, Eprist. IV, 27: " lemma sibi sumpsit (Sentius Anguriuus) quod ego interdum versibus ludo. Atque ateo iudirii mei te iudicem faciam, si mihi ex hor: ipso lemmate serumdus versis occurrerit; nam ceteros teneo, et ian expli॰ni:

\footnotetext{
Canto carmiua versibus minutis,

His wim quibus et meus Catullus

Et Calras veteresque. Sorl quid ad me"

Unas Plinins est mili priores.

Mavalt versiculos foro relicto

Et quяerit qual amet putatque anuri.

Illo o Plinins. ille quot, chatones !

I nunc, gquisquis amas, amrie noli.
}

I Catoni i quali si meravigliavano che Plinio (ille Plinius, ille!) scrivesse cotali versiculi erano forse più severi di coloro, dei quali egli dice (Epist. $\mathrm{V}, 3$ ) "extitisse etiam quosdum qui seriptre quidem ipsa non inprobarent: me tamen amice simpliciterque reprehenderent quod haec scriberem recitarcmque ». - Anche Petronio, e per la merlesima ragione se la piglia coi Catoni (Sut. 132): "quid me constricta spectatis fronte. Catones? ». Ma se l'etronio ricorre ad Epicuro, the amare inssit, Senzio Augnrino, per significare esser fatale l'amore, dice in tono di sfida: l mue, quisquis amas, amate noli: 
parla dell, slle tenui aspirazioni li vita e di poesia (1). Erli cui piconla parte dell avito patrimonio i rimasta, e che nun ha da vantare tra $\mathrm{i}$ suni antenati trofei di vittoria (r. 5is-sti). ruol reguare per l’ ingegnu poetico, ai wail banchetti, tra le fanciulle, enl assopirsi tra i fiuri, egli, cui il diu d'amore tuceo del suo dardo (r. $57-60)$. Segue un malnifico inno alla grandezza della poesia rergiliana (bi-so). Ma cerli, l'mperzio, si accontenta delle temui poesie It amore, le yuali non sammo ingratre legenti (81). E yui somo citati $\mathrm{i}$ moni dei poeti d'amore: Varrone che ludebut per lestearlia, rinè cantio lusus amorosi, il lascion Catulln. per i cui cammi Leshia o più fimosa di Elena, e il lonton Lalon che queste medesime cose confessio mentre

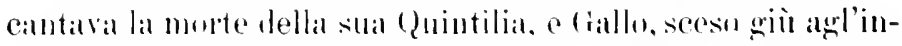
feri a lavarsi le ferite d'amore per la bella licoride (2):

S5) Hatec quonue perfecto ludebat Iasome Varro.

Varmo Leucaliae maxima flamma suate.

llane qunupe laseivi cantalunt seripta Catulli.

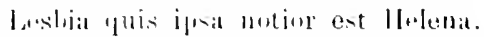

Ilane crian decti ronfessant pagina Calvi,

(9) Com ranteret miserae lunera Muintiliate.

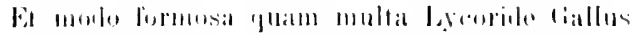

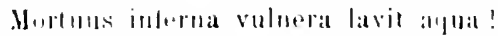

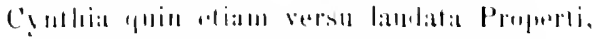

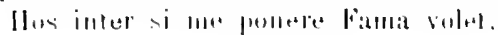

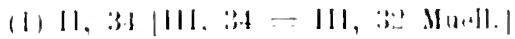

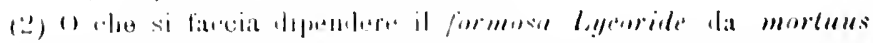

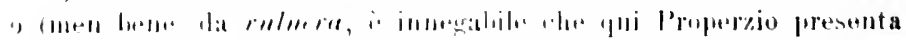

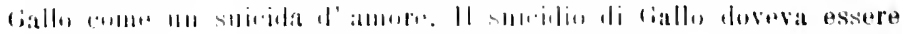

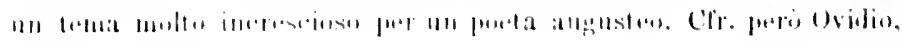
Tirel. II. 14:2-1:

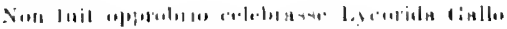

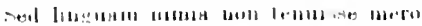


Ma che cosa mai confessara Calro nelle sue elegie per la morta Quintilia: La risposta ei viene da $11 n$ passo di Ovidio, che par quasi una parafrasi dei versi properziani teste ardotti; ell amplia di molto la lista dei poeti licenziosi, aggimgendo Ticida, Memmio, Cimna, Anser, Cornificio, Catone (1), Ortensio, Servio, Sisemna, ed argomentando quindi (r. 40): "Quis dubitet nomina tanta sequi?" Ah! i cattivi esempi!

Di Catullo e di Calvo così dice in questo lowogo Ovidio (Trist. II, 427 sgg.):

Sic sua lascivo cantata est saepe Catullo Femina, cui falsum Lesbia nomen erat. Nee contentus ea multos vulgavit amores In quibus ipse snam fassus adulterinum. Par fuit exigni similisune licentia Calvi, Detexit variis qui sua furta modis.

Dai due passi, di Properzio e di Ovilio, risulta dunque che Calvo fu licenziuso poeta, e che, come Catullo cantò le proprie infidelti a Lesbia, anche Calvo canto i proprii furti amorosi, e li comfossi anzi appunto nelle clegie dedicate alla morte della sua Quintilia. Da qualche indiziu, che or ura diremu, risulta che in puesta elegia era indotta l’ombra di Quintilia, che rivulueva la parula al poetal al allura si presenta spontanen il paragone con la elegial properziana $\mathbb{W}\left|V^{\top}\right|$, 7 , nella quale anche i presentata l'ombra di Cinzia, che rimprovera il superstite puetal. Qui Properzin rammenta in modo licenziosu i suoi furti

(1) E vioè, vertamente, l'antore di un poemetto sopra Diana, su cui vedi il framm. di Elvin Cinua (pr. Snet. De gramme 109 R.); Saecula permaneat nostri Dictynna Catonis. 
amorosi (15 rigiluris furta Sulurae), quando Cinzia calamblosi geio con una fune dalla tinestra sili carleva al collo,

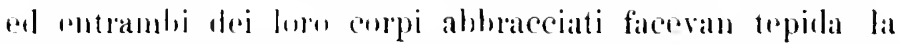
terra: rammenta, o si fir rammentare da Cinzia, $i$ suni giumanrenti li ferlelti, che $\mathrm{i}$ venti strapparonn vial (201-202) e l'abhamlono di Comzia lasciata alla merec di schiavi e schiare malvagie (2:3-4s): te fa che l'ombar amata gli sia pia ti generusi perdoni (6!)-70):

Sic morti lanrimis vitae sanamus amores:

Celo rege pertidiae crimina untra tuae.

Infine Cinzia grli esprime lo sue rulontii supreme o si dilegna, dopm arergli ammumziatu: Or sii pme di altre domne.

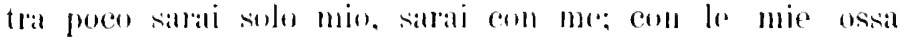
premerie lo tur confust coll !e mir (9),-91). - Chi comsi-

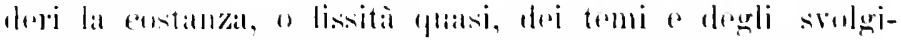

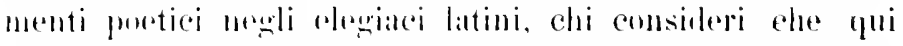

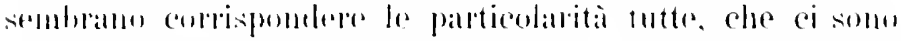

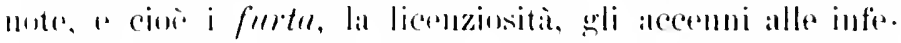

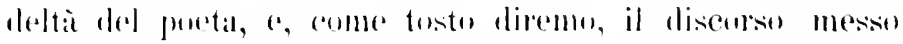

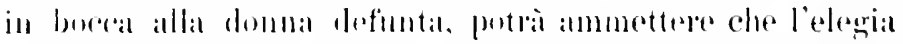

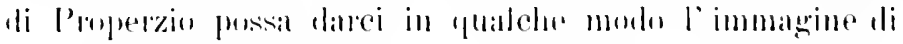

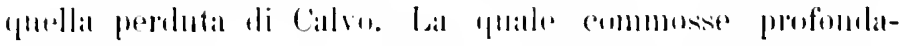

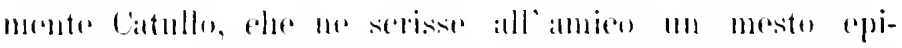
gramma (1). I versi defl' epigramma ci fammu ravisare

(1) rarme XCVI:

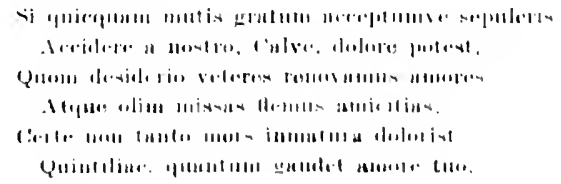


qualche altro rapporto con la elegia sopra esaminata di Properzio.

Da essi risulta che l'amore di Calvo per Quintilia era stato da molto tempo troncato (v. 4 missas amicitias), e che solo la morte lo rinnovara (renoramus), dando ad esso tributo di lacrime (flemus). Così appunto Cinzia era stata da molto tempo abbandonata da Properzio (IV, 7, 23-34); ma le lagrime della morte rintegrano gli amori della rita (ivi, 69): Sic mortis larrimis vitae sanamus amores. Gli ultimi due versi poi dichiarano che ora certo Quintilia laggiu, più che dolersi della propria morte immatura gode dell'amore di Calvo. É un pensiero ispirato a Catullo dalla elegia stessa di Calvo per Quintilia. Giacchè $\vdots$ impossibile non mettere a riscontro con quei versi di Catullo un frammento superstite di Calvo, nel quale è detto che forse il cenere stesso può di ciò godere:

fr. V Forsitan hoc etiam gaudeat ipsa cinis.

Clii parla qui ì probabilmente Quintilia stessa e cioè l'ombra della defunta amante. Giacchè in altro frammento, ove è anche rinis, detto a denotare defunti, il discorso è in prima persona:

fr. VI. ... cum iam fulva cinis fuero (1).

E i lue frammenti sono inseparabili.

(1) I due frammenti ci sono stati conservati da Carisio (I, 101 Keil) a cagione di rinis femminile (anche in italiano cenere è rimasto maschile e femminile; il seconto (cum iam fulva cinis fuero) anche da Nonio 19s, che per rimis femminile cita anche Cesare e Catullo, e che ha invece di fuero, fueris, o secondo altri codici fuerit. Il fr. può essere o la tine di un pentametro o il principio di un esametro. 
"Quamdo jo sarò diventata fulval cenere > e forse anche di cii pui esultare la cenere stessa sembrano il principin e il tine del discorso che l'ombra fa al superstite. Forsir, appena dopo la morte, essa a lui si presentavit a commettergli, come Cinzia a Properzio, le volonti estreme, e prima fra tutte, il pio ufficio del rogo; e finiva col dire che il suo conerr arrebhe esultata del rinbuvalo ammere di Calvo.

$$
* *
$$

Di un componimento poetico di Calvo sopra la favola li Io abbiamo expressa menzione e qualche citazione dai grammatici (1). Cle si tratti di un epillio è quasi certo, henche mon ne rimangat attestazione fagli antichi.

Ma in questo caso deve purr invocarsi l'atfinitio di ten-

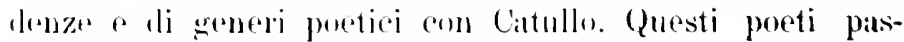
silvalun danli rpiglammi satirici ef angressivi agli epitalamii gontili el ai poemetti li argomento mitologico e di earatere rention a sentimentale; mal si siprebhe per essi

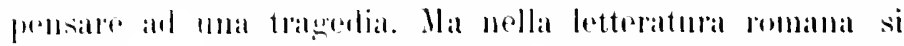

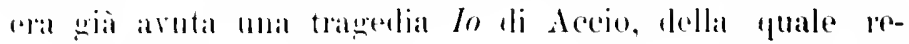

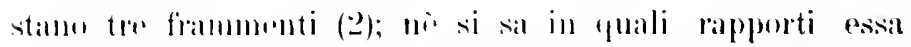

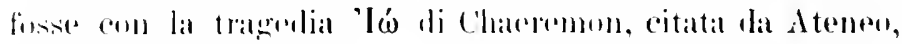

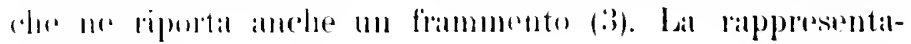

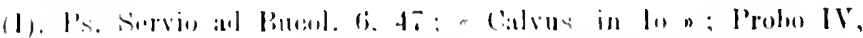

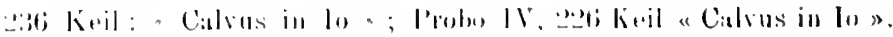

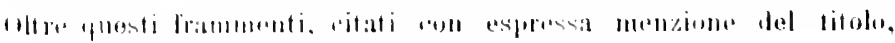

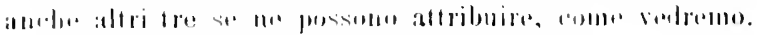

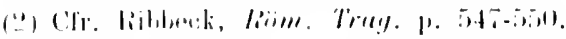

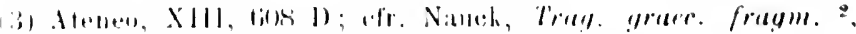

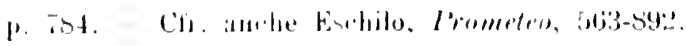


zione di Io vagante es citata come figura di tragedia da Orazio (Art. poet. 124) (1). Ma la favola per il suo contenuto patetico. per la rarietà dei casi dolorosi, per l' elemento stesso della passione di amore e del furore geloso, che vi predominara, ben si prestava alla elaborazione degli Alessandrini, e specialmente di quei poeti, che di proposito arevan trattato le favole delle trasformazioni; ed a qualcuna di essi fu ispirata probabilmente la trattazione che della favola troviamo nelle Metamorfosi ovidiane (I, 568-750), e nella XIV delle Eroidi (85-108).

E nota la farola di Io, figlia del re d'Argo Inaco, che per essere stata amata da Giore suscitò l'ira furiosa di Giunone, la quale la trasformò in vacca muggente e la afficó in custodia al Argo. Ma Giove fece da Mercurio nccidere Argo; ed allora Giunone fece inseguire la vacea Io da un assillo o da una furia, e fece che errasse per tutta la terra, senza avere wai posa, senza prendere mai sonno. In Egitto finalmente essa riacquistò la forma umana, e partorì Epafo, che fu re di quella terra e foudi Menfi. Molta rarietà aveva naturalmente la farola dall'ana redazione all' altra, specialmente circa l'origine di lo (2) e

(1) Antichi critici pensarono ehe anche l'Io di Calro fosse tragedia; cfr. Weichert, P'oet. Lat. Rell. P. 144: "Qui tragoediam Ius nomine a Calvo scriptam opinantur, eos miritice allucinari malnifestum ost, eorumque opinationem contilentius refellere poterat Ellemitins in Proleg. in Ciceronis Brutum p. CXXV .

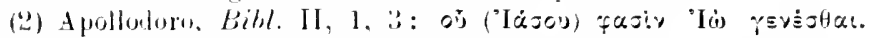

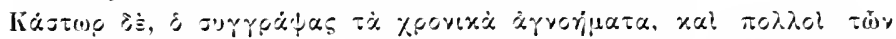

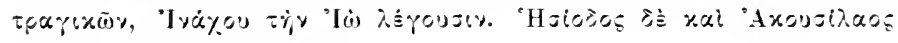

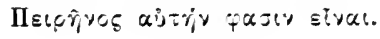


di Argo Panopte (1). Ma le linee principali della favola somin queste (2)).

All' mo o all' altro particolare mitico della leggrenda si riferiscono i rersi cle del poremetto di lalso ci sono rimasti. Alla trasformazione in racea si riferisce il bel verso conservatoci da Servio (3) :

fr. V'll. A, virgo infelix, herbis pasceris amaris.

P'uó essere mi' apostrofe fatta dal preta stessio alla sua moina, mentre si accinge a narrare l' amore di friove e la presecuzione di lui alla fancinlla ritrusia (efr. Ovid. Ifot. I. 5s!!-600). il verso eluhe l'omure di mal doppia imitazione rergiliana:

Erl. VI. 47:

A. virgo iufelix, quate to dementia reput:

$\operatorname{Iri}, 22:$

A. virgo infelix, th mune in montihus erras.

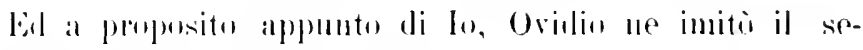

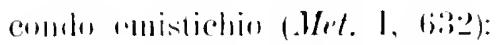

Fromblous arboreis et amotra masritur herbat.

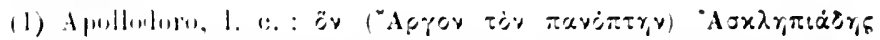

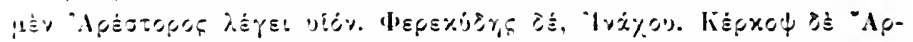

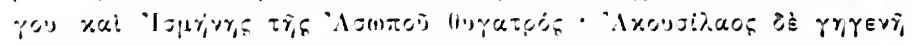

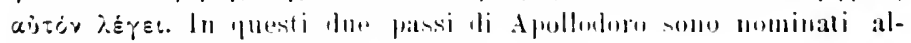
ronie di quelli, rhe averatn trattaln il mito.

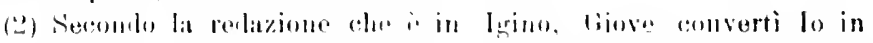

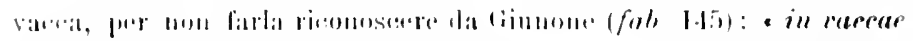

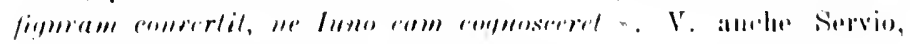
at rerory. 111. 15is.

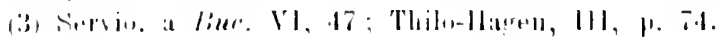


Oridio stesso, altrove, col medesimo movimento poetico di un' apostrofe si rivolge ad Io:

Heroid, XIY, 93:

Quid furis, infelix: quid te miraris in umbra?

Ad L, presara delle imminenti sventure, piuttostochè alle furie gelose di Gimone sarà da riferire il frammento (1).

fr. VIII. Mens moa dira sibi pradicens omuia vecors

Naturalmente il dira è da riferire ad ommia, non a mens.

Ad Argo vinto dal somro aecenua il frammento (2).

fr. IX. Cum gravis ingenti conivere pupula somno.

Pupula i singolare poetico, e qui vale - whli ucchi . Nel verso seguente dovera trovarsi imripit, o qualche cosa di simile. Conivere è qui infinito della $3^{n}$ coningazione, come frrrore presso Properzio e Vergilio (3); ed in grazia

(1) Probo, IV, 234 Keil, eita il verso a proposito lel giambieo sibi. Il Weichert (1. 144) proponeva, invece di ommia, omima. Ma omnia sta bene: la misera forsennata, nol momento the comincia al accorgersi che le sue fattezze lentimente si trasormano, prevede tutte le sventure piu terribili.

(2) Presso Prisciano, I, 479 Keil. Arruta la congettura li Nic. Heiusius, urgenti per ingenti. Ma ingenti sta bene, e meglio si accorda col gravis, che indica la pupilla appesantita dal gran sonno.

13) Fervĕve Prop. II, 8, 32; Vers. Feory. 1, 456; Aen. IV, 409 e 567. A poposito di eommere Prisciano, 1. ¿. ha: * Caluns conirere infinitum secundum tertiam eoniugationem correpta paenultima protulit: Cum gravis ece. . 
appunto di tal forma ei è stato eomerrato il verso. Mercurio profittio del somno di Arero per neciderlo.

En altro verso superstite si riferisce ai lunghi tormentosi errori di Io vasante per tutta la terra e la rappresenta che s' ineammina a fredde spiagrie. Il verso è da Probo (1) riportato nella forma: "Frigide inul relevis erryatar ristimis ora s, ehe non ba sensol. Le congetture sono parecchie (巳)

Quella che ne par più vieina alla trarlizione manuscritta fu proposta dal Batehrens (3):

fr. X. Frigida iam coleri superatast Bistonis ora

bistonis (- idis) ì nominativo femminile: Bistomis

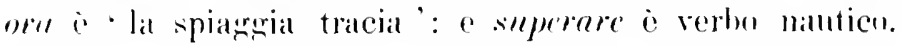
bistonis ma si trova del resto anche nella XVI Heroide ovirliana, v. 240: kit tuta a hello bistonts ora, we anche hisonnera quindi riconosece in bistonis il nom. dell'arg. femminile.

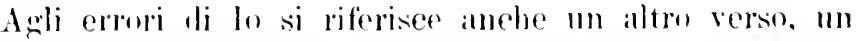
hel verse, che anche ci $\dot{a}$ satu comservatu da lan zrammaticu. e per ma citazinne arammatieale. Lo si lamenta

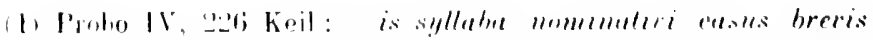

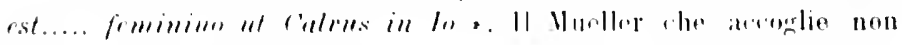
una forma li mom lemm., ma una lomma di gend. Bornsthemis,

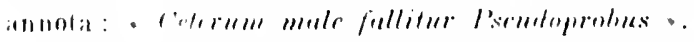

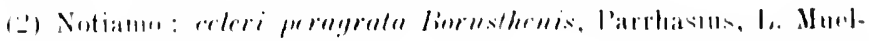

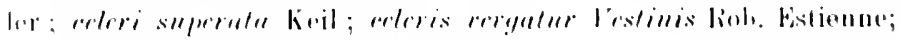

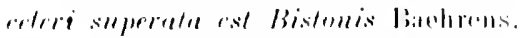

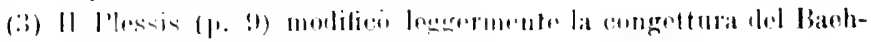
mus: aleri stmpralme bistmis orn. 
del suo perenne vamare: essa non la riposo mai, mentre anche il sole si riposa:

fr. XI. Sol quorue perpetuos meminit requiescere cursus (1).

Requiesrere ha qui senso attivo e causativo? "Il Sole si ricorda di far cessare il suo perpetuo viagrio?" Oppure: «Anche il Sole si rammenta che i suoi perpetui cammini hamno riposo!" Questa secomba interpretazione ci sembra più naturale; e wli altri passi ardotti per gillstificare requiescere tramsitivo non sembrano suffragare I' interpretazione (2).

Al poemetto Io appartiene senza dubhio l’altro frammento:

fr. XII. lartus gravido portahat in alvo

In era incinta di Giove e doveva partorire Epafo (3). Due grammatici (4), a proposito del passo di Vercilio

(1) Ps. Servio a Verer. Ecl. VIll, 1 (IIl, 1. 92 Thilo).

(2) Nel passo di Vergilio qui sopra citato ft mutata suos requicrunt flumina oursus) il suns mrsus lipende da mututa, piuttosto che da requicrunt (ace. alla ireca). Il Forhiger. a '।. I., accetta il signif. transitivo di requierunt, citanto il verso di Calvo. e Ciris. 232: Calpurnio. cel. I1, 15; ma v. Jacoh a l'roperzin III, 15 (II, 22, 25 (1). 163 seng.): Impriter Atrmenae geminas requieverat Aretos.

(3) Il frammento è citato dal grammatico Carisio, per locumentare l'uso di alrus mascolino, del quale usu Carisio stesso cita altri esempii di Cinna. Laberio. Attio (afr. Carisio 1, so Keil).

(4) Matrobio, Sat. 111, 8, 2: * nam et apud Calrum Aterianus adfirmat legendum pollentemque deum Tenerem, non deam. Servio, ad Aen. II, 632, vol. 1, p. 312 Th.: "utriusque serus participa- 


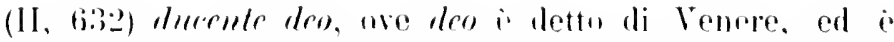
aloperato quindi come nome di genere comme, citamo un altro passo di Calvo:

fr. XIII. pollentemque doum Venerem.

Che qui prè denm sia accusativo singolare e quasi sicuramentr da escludere. Si trattria piuttostu della furma demm dol zentitivo plurale, siroume griai videro il Voss e molti altri (1). Il versu signitica dumpue che Vonere ha potere anche sugrli dei; efr. Sufucle, (fr. s55 Sauck ${ }^{2}$ ), a proposito di Afrodite:

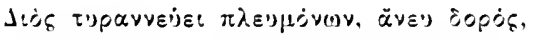

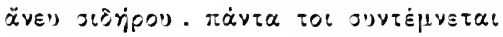

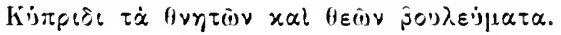

Circa pollens col senitivo efr. Platuto, C'11\%. I, 2, 221: rimi pollens Liher (2).

tinnem habere mrmima. Mum ait Calrus: p. d. I. ". Tanto Ate-

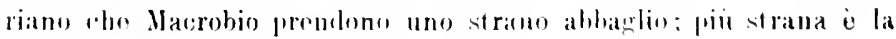
spirgaziono li servio.

(1) Voss, a Cat. 1). 25!) : v. uli antori ritati clal Burmanu aul Anth. Lat. I, p. diro. Interprotano uronitivo anche il Mullor, p. S4 on il Plessis p. 7. Drum non i perio forma sincopata da demmm, spierazione glotfologrioumente impensibile: $i$ la forma col suflisso del gen. $-\pi m$ (三

(2) l'ii frequente is in tale uso potens: traz. ('arm. 1, 3, 1 :

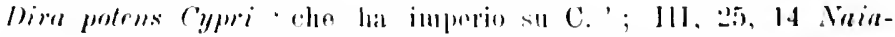

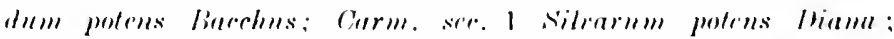

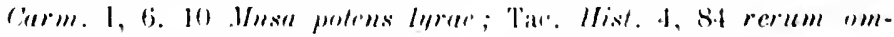

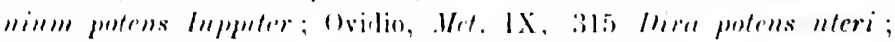

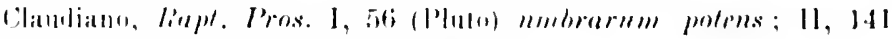
(l'alla(s) tubarmm armmenmque polems. 
Anche questo frammento probabilmente apparteneval al poemetto Io; Giove era stato preso d'amore per Io, perchè Venere signoregrgia gli stessi dèi.

$$
\stackrel{*}{*} *
$$

Un frammento di argomento mitologicn, e che riguarda propriamente Cerere, ci è stato conservato da Servio (ad den. VIII, 58), del quale adduciano il passo: " Alie dicunt favere nuptiis Cerevem, quod prima mupserit Iori, ct condendis urbibus pracsit, ut Calens doct:

fr. XIV. Ft legres sanetas docnit et cara ingavit

Corpora conuhiis et magnas condidit urbes (1).

Si tratta di Cerere Thermophoros, considerata come la dea del rivere cirile, che insegnò le leggi, e importó l’uso delle nozze e quindi costitui le famiglie e le aduuo in città.

Primo l'Osann (2) pensò che questi versi appartenessero ad un epitalamio; e di tale opinione fu pure il Mueller (3). Vediamo di ciò breremente.

Anzitutto è motevole che secondo una credenza romana Cerere era considerata come nemica delle nozze; ed anzi durante le cerimonie di Cerere i homani si guardavano bene di nominare il padre o la figlia, il che poteva ram-

(1) Notevoli le due particolarità metriche di questi versi : docuit con l' $i$ lungo e cumubies con l'n breve: v. Plessis, op. cit. 11. 5-6, e L. Mueller, De re metrica $2(1894), \mathrm{p} .302 \mathrm{sgr}$.

(2) Ad Apul. de Orthogr. \$2, p. 17.

(3) Cfr. p. 84: Ex epithalamiis etiam videntur sumpta haec. 
mentare alla lea il rapimento di Libera (o Proserpina) (1).

Quale che siasi il valore da dare a questa notizia, certo a che Cerere, come Tesmophoros presso i Greci, era considerata come una dea nuziale: ed anzi cra nol rito che si portasse nelle cerimonie nuziali una face in onore di Cerere (2). D'altra parte si celebrava in Roma anche una festivitio, letta nozze di Cerere o no: ac di Oreo (3), e che fil pensare anche allo sposalizio di Plutone e di Proserpina, che si celebrava in (irecia nell estate (4). Pui dunque il frammento sopra ardotto riferirsi al coro di una pompa nuziale, che portando le faci alla dea, celebra $i$ benetizii da essa largiti all’ umanità.

$$
* *
$$

Espressamente da un epitalamin i citato l’altro frammento :

fr. Xr. Hesprium ante iubar quatien.

(1) Servin, Ad Ir"l. IV, 5s a Alii licunt... Cererem propter raptum tilias nuplias exsereratam... Et liomae rom Cegeris sacra dant ohservatur ne quis patrom aut filian nominel, fuod fructus matrimonii fre liheros sonstet , Percio Cerere fu stimata anche lea lei divorzii! Servio, Al Arn. Ill, 139: "quidam disunt diversis muminilus vol lone wol male faciendi potestatem dicalam, ul Venuri eomimeria, Comeri divurtia....

(2) Panlo rpit. 1. S5 \& farem in muptiis in lonnurem Coreris prateforehant \$. Clr. Simpio ad Aru. IV, 5s.

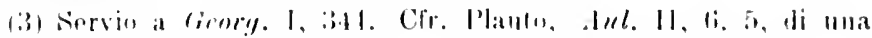
errmonia musialo in eni mansa il vino: "Coserimo has fanturi

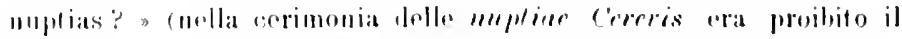
vilin.

(1) Cfr. P'reller, (iricrh. Wythot. ${ }^{2} 1,612$. 
Il passo, che ì un frammento di esamctro dattilico (1), ¿ citato da Prisciano (2) per la roce inbur. Hesperium iubre è la stella Espero, al cui sorgere si celebravano le nozze, e la cui menzione non manea perciò mai negrli epitalamii o negli accenni a cerimonie nuziali (3).

Un frammento citato da Carisio (t) per la roce munui risulta di un yliconeo e di un ferecrazio, metro adoperato da Catullo nel carme LXI, che e l’epitalamio di Manlio e Giulia; ed anche il passo di Calvo sembra appartenere ad un epitalamio:

fr. XVI. Lilium vaga candito

Nympha quod secet ungui

Lilium fu supplito dal Broukhuys (5), e la sua congettura fu generalmente accetta. Dei gigli colti da tenera donzella dice appunto Properzio (1, 20, 39):

Guae (lilia) modo decerpens tencro pueriliter ungrui.

Il frammentiuo di esametro

fr. XVII. $\quad$... hunc lanto munere digna

(1) Osserva il Plessis (1). 5) che potrehbe pensarsi anche ad un verso anapestico.

(2) Prisciano, I, 170 Hertz: "Calvus in epithalamio".

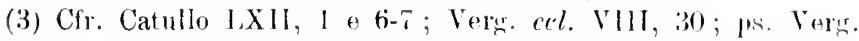
Coris 348-351, ece. V. altri molti jasis nel min lavono Hesperes Oitaios in heudicouti Jst. Lomb., vol. XL, 1907, 1. 975 sgg.

(4) Carisio I, 147 Keil.

(5) Nota a Properzio I, 20, 39. 
¿ citato da servo (1) per il verbo attivo (invece del depunente digmari) reputar downo'.

E) molto probabile, come gria fu congetturato da L. Mueller (p. S4) che anche questo frammentino appartenga ad epitalamio.

La scena sara quella medesima del carme LXIl catulliano. I due enri, dei giovani e delle donzelle, aceordatisi in fine. si rivolunono alla sposil, e la esortano a non resistere allo sposo. Cfr. Catullo, IAXIl, 5!) "At t" te pugmu

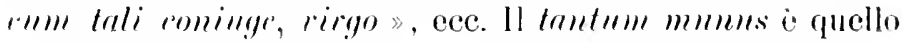
di cui ivi appouto parla, con grazia biricchina, Catullo, ․ 61-65.

$$
* *
$$

Ahhiamo "un altr" frammento di Calon, un enrlecasillabo

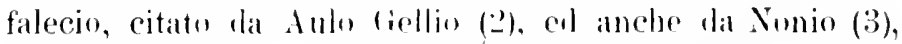
ove perio il nome dell’antore ce citato come finllus, facile corruziene di calrus:

fr. XVIII. Durum ras fucis ef lateriosum.

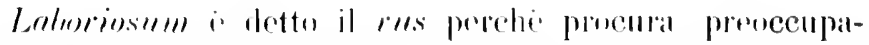
ziond "latiche (1). A chi en a the si riferisea il fram-

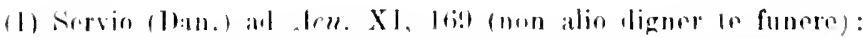

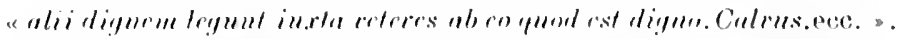

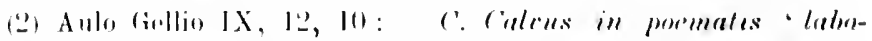
rinsus dirit, mone ut rolyo dieitur, qui laborot, sed in quon laboratur: durmm..... s (i ms.r. di tiollin hanmo fugiet o fugfi sed).

(3) Nonio. 1:3:3: a ciollus pormalis: durnme rms fugile laborin-

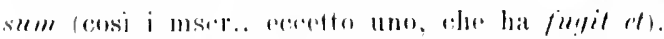

(4) Lahoriosus in senso passivo; efr. Catullo 1.7 Marziale X. 10.1, 13. V. sopra il passo apportate di Gollin, the nel $\$$. 1 dul 
mento, non è possibile dire: ed è vano quindi arventurarsi a discutere se si abbia a leggere fugis o fugit o fugiet o altrimenti.

Ad un carme di Calro appartenera certamente il passo, che è rammentato, ma non citato, da uno scoliaste vergiliano, il cosidetto scoliaste Bernese (a Georg. I, 125): "dicunt lovem conmutasse omnia, cmm bonus a malo non discerneretn, terra ommia liberins ferente, quorl Calrus ranit. Inmilius dicit» (fr. XIX). Il canit ci fir certi che l'autore roleva riferirsi ad un carme di Calvo.

Anche Calvo dunque descrisse la primitiva feliciti del secolo d'oro, e i mali apportati sulla terra Tove sul domino: mo dei temi obbligati della poesia latina. Ma in qual componimento, e di qual genere, egli abbia trattato tale soggetto, non è facile congetturare.

Allo stesso scoliaste Beruese dobbiamo il ricordo e la citazione di un altro passo di Calvo, ma il passo è riportato in tal maniera che è disperata impresa ravisarvi una forma metrica: e d'altra parte la citazione stessa del grammatico sembra escludere che si tratti di prosa. La citazione ì nel commentario al verso delle Georgiche (II, 94); "temptatura pedes olim rincturaque linguam». Lo scoliaste annota: "Hos rersus a Calro poeta transtulit. Ait enim ille: lingna rino temptantm et pedes" (fr. XX). Li hos ressns pui far pensare che l'imitazione o riproduzione reroiliana si estenda anche al di li delle

capitolo medesimo areva viì spiegato: "laboriosus et qui laborat et qui labori est $\$$ 
parole addotte: di quella riproduzione il grammatico avrobbe apportato solo il più calzante esempio. Ma nell'apportarlo egli, forse citando a memoria, tralasció fualche parola. il che fece scomparire le tracee della formal metrica (1).

(1) É molto dublio se il passo. Jel quale abbiamo gria disensso,

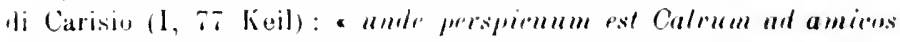

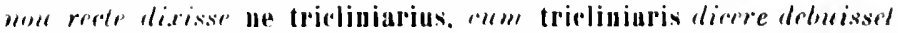

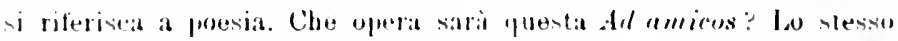

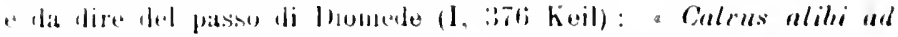

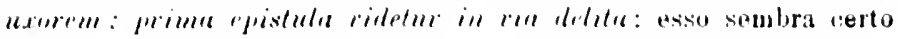

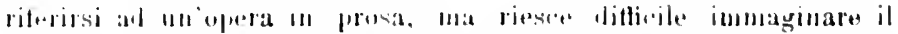

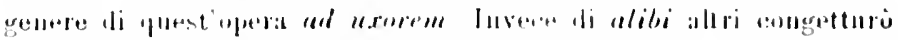

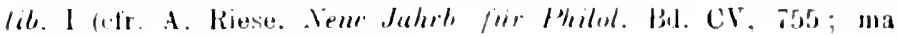
v. Keil. Vir. J. al luoso cit.). 
II.

IL POE'TA RLIIO CINNA 


\section{AVTERTEN\%A}

Pin lo citazimi, fatte in forma athomiata, coi suli nomi degli antori (ad es. L. Nueller, Barlarens, exp.l, si tenwanu presenti le indiraziuni late a p. $:$.. 
Di C. Elvio Cinna fecero spesso menzione gli antichi a proposito della morte di Cesare, e narrarono un tragico equivoco, del quale Cimna appunto rimase vittima. Egli, che era stato fido amico di Cesare, volle partecipare agli estremi onori, che si rendevano al rogo dell' ucciso: ma fu dal popolo scambiato, per errore del nome, con Cornelio Cimna, che avera il giorno prima parlato contro Cesare e che eral tra i congiurati (1); e fu dal furore popolare miseramente trucidato, e ne fu portato in giro,

(1) L. Cornelio Cinnat, pretore nel 710. Narra Appiano (B. $e$. II, 121) che egli nella concione, gettata la veste pretoria, perchi ricevuta da Cesare, chiamò Cosare tiranno e tirannicidi gli uceisori; siechè il giorno dopo, mentre si recava all'adunanza del Senato, convocato da M. Antonio nel Tempio di Tellure, fu assalito a pietre dai veterani di Cesare, e poco mancò che ei ne perisse (App. B. c. I, 126). Questo personaggio è il medesimo L. Cinna, altamente lodato da Cicerone nel Dic. 710/4t per aver rifiutato la provincia offertagli da Antonio (Cic. Philipp. HI, 10, 26), come già videro i critici antichi Garaton. a Cic. l. cit. vol. II, 1. 49) e tra i moderni h. Soluwatue (Mhilologyes, XIVII, 1. 1699).

C. PABCAL

4. 
orrendo trofeo di sciagurata rendetta, il capo confitto ad un' asta (1).

Questo Elrio Cinna è il poeta amico di Catullo? Il solo Plutareo lo indica come poeta: Kívras ro:ntix.s $\dot{\alpha} v \dot{r}_{f} \rho$, e in uno solo dei luoglii. in cui narra il doloroso avvenimento (Brut. 20). Suetonio nel cap. 85 (Iul. Caes.) to chiama semplicemente Elrio Cinna, ma nel cap. 52 nomina un Helins Cimna tribunus plebis; Appiano to

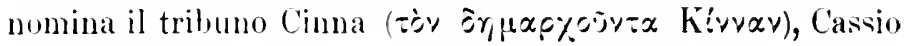

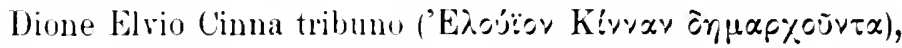
Valerio Massimo to chiama C. Itelins Cimna tribunus plebis (2), Plutarco nella Vitu di Cesare (c. tis) lo chiama

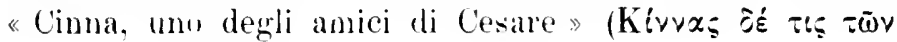

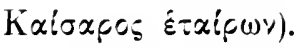

Il Ribbeck (3) a proposito di tali racconti osserva, esser verosimile che Plutareo si sbagli : priche egli solo indica l'ucciso come poeta; le altre fonti, secondo lui, consentono la naturale ipotesi che sia stato Cornelio Cinna, il congiurato, a carlere vittima della rendetta popolare. Dovrehbe così eliminarsi, e reputarsi una mera inven-

(1) Suetonio, Cars. Si: : Plels statim a funere (Caesaris) and lomum Bruti et Cassii cum facibus totendit: atyue aegre repulsa, obvium sibi Helvium Cinnam, per errorem nominis, puasi Cormelius is esset, quem graviter pridie concionatum de Caesare requirebat, occidit, caputque eius praelixum bast ale eircumtulit, ipuesto Elvio Cinna e chiamato da Suetonio poeo prima, s. 5), tribumus plobis, al quate Cesare avrobbe commesso l' incarien di presentaro ma logge che permettesse la poligamia, liberormm quarrmelormm

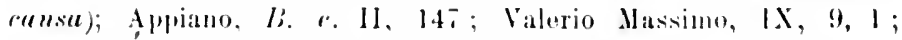

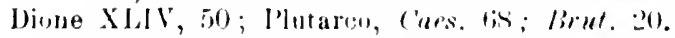

() de indiazioni doi passi vedi nella nota preredente.

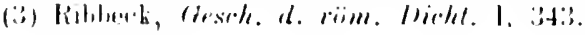


zione, tutto il racconto dello scambio di persona, e dell'equivoco sciagurato in cui cadde il popolo; ma, a prescindere dalla poca probabilità di ciò, sta di fatto che Cornelio Cinna era ancor vivo nel Dicembre $710 / 44$, perchè allora fu lodato da Cicerone (1) per aver rifiutato la provincia offertagli da Antonio; non poteva quindi essere stato ucciso nel Marzo $710 / 44$, durante i funerali di Cesare (2).

Con molta probabilità la persona così miseramente perita durante le cerimonie funebri in onore di Cesare fu proprio il poeta C. Elvio Cinna. L'errore del nome è spiegabile nel tramestio della furia popolare; e che Cornelio Cinna fosse ricercato dal popolo e che corresse in quei giorni pericolo, risulta da alcune fonti (3). Assolutamente da escludere è che nel passo di Plutarco (Brut. 20) vi sia errore di lezione (4); molto poco probabile è che l'Elvio Cinna tribuno debba ritenersi diverso dall' Elvio Cinna poeta, e che Plutarco per la identità del nome abbia fatto confusione. Ciò ritenne un dottissimo critico, il Weichert (5), e vi fu indotto da clue ragioni, che non

(1) Vedi la nota prima di questo lavoro.

(2) Ciù fu già osservato dallo Schwabe, in Philologus vol. XIVII, p. 169.

(3) Suet. Cues. 85 a quem (Cornelium Cinnam) grariter pridie concionatum de Caesare requirebat (plebs) \$. Della concione violenta contro Cesare fa ricordo Appiano (B. c. II, 121), ed altrove (I, 126) del pericolo corso di essere lapidato dai veterani di Cesare.

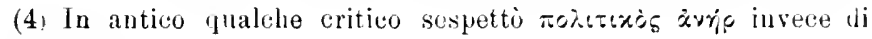

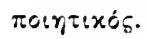

(5) Weichert, Ise C. Helvio Cinna poeta in Poetarum Latinorum ecc. Keliquiae, p. 154. Già molto prima di lui il Torrentiur, Comment. ad snet. (Antwerp. 15is) p. St. Tra i recenti lo Schanz, Gesch. der röm. Litt. I, § 107. 
sono scere di qualche valore, ma neppur sono inespugnabili. 'Tra i componimenti di Cimna si cita, come vedremo, un Propempticon Polliomis, di cui rimane qualche verso. Tal componimento si riferisce da molti, secondo l'antica ipostesi del Voss, alla spedizione conclotta da Pollione contro i Partini, gente della Dalmazia, nel 714 di R. Se it riferimento fosse fuori dubbio, dorrebbe ritenersi Cinna ancor vivente nel 714. Ma il componimento puó anche riferirsi, come redremo, ad un viaggio anteriore di Asinio Pollione nella Virecia (1).

l'iù grave è l'altra ragione tratta dal verso li Vergilio, erl. IX, 35 :

Nam neque aldue Varo (2) videor nee dicere Cinna Digna

Ora i fuor di dubbio, por le allusioni personali che vi si contengono, che l'eglnga IX di Vergilio sia da riferire al 714 . In quell'anmo dumque il poeta mantovano facera questo elogio di Cimm. Par difficile ammettere che mo complimento di tal gencere debba intendersi indirizzato a 1 m morto. lo eompremele tutto il valure di questa

1) Cfr. A. Kiessling, Me C. Melrin Cimna preta nello Commentationes in homoreme Th. Mommesemi, 1. :35.".

(2) Varo legro seconda i conlici e riferisco a Quintilio Varo: chr. le mie Commentationes erry. 1. 3S-39. Ad ogni molo ancho se si avesse a legrore larin (Heyne. lablewig, forbiger, Benoist, kiaples, libheek, eve.', il ragionamento per phesta parto non cadrebbe. Nel passo della mia opera sopra eitata nen i peró da tener

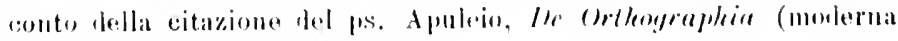

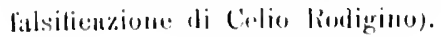


osservazione (1). Ma d'altra parte in questo caso occorre pur procedere per esclusione delle ipotesi più improbabili, facendo rimanere in piedi la neno improbabile. Or mi parrebbe strano che tutto quel racconto, determinato $\mathrm{e}$ circostanziato nei suoi particolari, sullo scambio dei due personagrgi di nome Cimna, e sull'equivoco popolare che condusse a tragica fine Elrio Cimna, fosse nato dal nulla e non corrispondesse ad alcun fatto reale; e piì strano ancora che contemporaneamente vivessero in Roma un Elvio Cimna tribuno del popolo el un Elvio Cimna poeta.

Meno improbabile di queste due ipotesi ci sembra l'altra, che Elvio Cimna tribuno popolare fosse anche poeta; che egli fosse nel 710 vittima del tragico equivoco, e che, anche quattro anni dopo la sua morte, Vergilio, sotto le spoghlie del pastore Menalca (2) attestasse non saper scrivere cose degne di Varo e di Cinna (3).

(1) Cfr. anche Schanz, Gesch. d. röm. Litt. I, § 107: * es scheint daher (Verg. ecl. IX, 35) dass wie Varius so Cinna damals (40 v. Chr.) noch am Leben war ». È però melto notevole quel che osserva il Kiessling (De C. Helvio Cinna poeta in Comm. in hon. Nommseni, 1877, p. 3553) che quei versi sono imitazione dei Teecritei (VII, $39 \mathrm{sg}$.), nei quali un simile elegio è diretto a Sicelida di Samo ed a Fileta, benchè all'epoca della composizione (lell' idillio VII di Teocrito Fileta fosse già morto.

(2) Già Quintiliano (Inst. orat. VIII, 6, 47) avverti che sotto il velame di quel nome si nascondeva il poeta: non pastor Menalcas, sed Vergilius est intellegendus».

(3) Forse la mienzione di Varo risvegliò al pensiero quella dell' intime amice Cinna. Giacchè dello strettissime legame che teneva avvinti in affettuosa consuetudine Catullo, Varo e Cinna ci fa testimenianza il carme $\mathrm{X}$ di Catullo anche il Varo di questo carme e del carme XX mi par probabile sia Quintilio Vare cremonese). 


$$
* *
$$

Dn resto sulla vita di Cinna ben poeo si sia. Che eyli fossere lella Ciallia Cisalpina ì possibile: ma mon è dato loterminare quale fosse la sua patria (1). Un debole indizio is a tal proposito quel che fu usservatu dal Kiessling. che cioce le iserizioni riguardanti la geus Hrlein sono fropuenti a Briria (2), rella quale citta egli suppose quindi nation il mostro poeta. Unica notizia sienra e che egrli prese parte, come Catullo, alla spetlizione di Bitinia, al

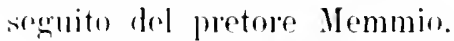

II pretore C. Memmio (3), pompeiano, fu amante di lettore greche, e tenti, oltre la irloria militare, anche quella aratoria e poetica. Al swo spiritu mordace aceonna sinetonio (4), of alla licenza dei versi snoi Ovidin e Plinio (5), mentre da Ciellio (6) si pus aromentare come fosse duro

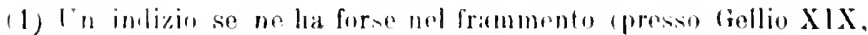

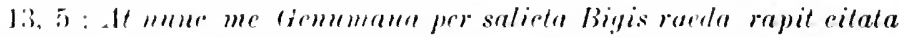
namis . Le citti principali dei Cennmani rano lerona, brixia (Cremona.

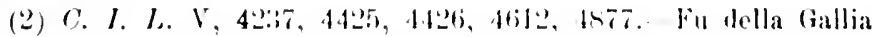
Cisalpina in quoll' ola tutla una mobile subiera di prosatori o di fueti; tra questi Catullo, furio libaculo, Quintilio Varo. - 11

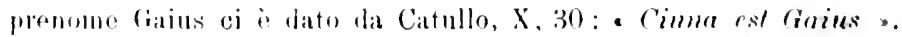
Circa il cogmome Cinna verli Zimmermann, freh. fïr lot. Lexiliograplice, Vi. stig), 71 .

:i) II rennomo fiemelles gli fu attribrito per equivoes eon

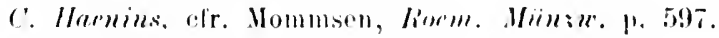

(4) Suet. ('uess. 49) o 73.

(5) Urilio, Trist. II, 4333; I'linio, Epist. V, 3.

(b) Cirllin XlX, 9. Rimane di lui un solo verso: Ardun ne nitous fortumae eseculere rlier (presses Nonio, 13.4. 
il suo stile poetico. Pare che nella rita privata fosse uomo di corrotti costumi (1). In politica fu avrersario di Lucullo (2), di Clodio (3), e di Cesare, contro il quale tenne parecchie orazioni (4), e che non mancò infine di adescarlo e conquistarlo (5). A lui il grande poeta della Natura dedicò la sua gloriosa fatica. Lucrezio rimase vittima di una di quelle illusioni, che sono comuni agli nomini di sturlio, e fu affascinato dalle arti e dalle seduzioni di questo uomo pratico del mondo, brillante e scaltro. Certo i rettami epicurei del grande poeta non valsero ad ispirare a Memmio tanta reverenza per la memoria di Epicuro, che egli s'inducesse a salvare le reliquie degli orti e della casa di lui. Giacchè Memmio in seguito ad un accusa di broglio (6) dovè infine ritirarsi ad Atene (7), ed ivi entrò in possesso di un terreno ore erano i ruderi della casa e degli orti di Epicuro, quella casa e quegli orti che il graude filosofo avera lasciato in eredità alla sua scuola. Memmio voleva senz'altro abbatterli per farvi nuove costruzioni. Cicerone pregato dagli amici epicurei intervenne per dissuaderlo:

(1) Cic. Ad. Att. 1, 18, 3 ; Suet. de ill. gramm. 14. Le espressioni presso Catullo X, 12 e XXVIII, 9, sono frasi triviali, la non prendersi nel senso letterale.

(2) Plutarco, Luc. 37.

(3) Cic. Ad. Att. II, 12, 2 ,

(4) Suet. Caes. 49 e 73 ; Schol. Bol,. in Cic. Pro Sestio 997 , in Vat. 317 e 323 Or.

(5) Suet. Caes. 73.

(6) Cic. Ad. Qu. fr. III, 2, 3.

(7) Cic. Ad. Att. V, 11, 5. Da Atene invano si tento farlo tornare (Ad. Att. VI, 1, 23). Forse vi mori. 
e il feer com mal lettera, tra seria ed ironica (1): ne si comsere con quale esito.

II (mmin prese parte alla querra Sertoriana, come questore di Cn. Pompeio (2), e nel b96 fu maudato in qualita li propretore in una spedizione in Bitinia (3).

A questa spedizione presero parte, nella coorte pretoria di llemmio, due poeti arrenturosi e desiderosi di fortuna, Valerio Catullo ed Elvio Cima. Giacchè alla coorte pretoria ela ascritto un certo numero di grovani. che circondarano il comandante la sperlizione, e secondo gl incarichi e shi ufficii si distinguerano in comrirtores ed appreritores. Aiutarano il comandante nej sunj lasori, trnevanu la corrispondenza, formavano quasi un consighlio intimo intorno a lui, davano pareri in questioni di diritto, $\therefore$ assilevano alla sua mensa, alleviando le fatiche militari con ricreazioni dello spirito. Altri averano ufficii più pratici e positivi: fornitori, collettori di rettoraglie, esattori li imposte e simili: tutti mezzi huoni per arricchire (1). Memmio, che si piccava di poesia. volle avere al stu sornito i lue poeti: rli arrise forse speranza che essi, " mun di essi, ne canterehbe le sperate vittorie? II

(1) Cir. Fam. XIII, 1.

(2) Cic. Balb. "2, 5).

(3) A fuesta spedizine, 0 al ritorno da usit si rifiriscono alrnni carmi atulliani: $X$. XXVll, XXXl, XlVI.

14. Cfr. Cicron", In Ferr. II, 10, 27: Comites illi tui delecti mimus rant tman: praefecti, scribar. accensi, medici, haruspices, pareones manus arant tuac. It quisplue maxime cognatione, aftinitate. nesersituline aliqua attingebat, ita maxime manus tua pu-

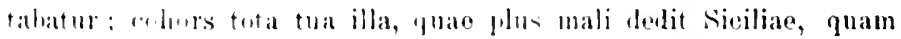
si antum cohortes fugitivormm fuissont, twa manus sine controuersia linit. 
ricordo di Seipione e di Emnio non era molto lontamo. Na Catullo, e probabilmente anche Cimna, non miravano che a far quattrini. Si può essere sicuri che Catullo non fece nè l'approvigionatore di viveri nè il collettore di imposte; egli probabilmente non feee, 0 non continui a fare, che il buontempone e il giovanotto del mondo gaio: e forse appunto per questo gli sperati guadagni non si videro mai. Catullo se ne lagnò amaramente e ne gettò tutta la colpa sopla Memmio: egli, secondo lui, li aveva corbellati tutti e non areva fatto nessun conto della sua coorte (ner faceret pili cohortem) (1). Pari disavventura del resto toccò in seguito ad altri lue amici carissimi di Catullo, Veranio e Fabullo, della coorte di Pisone (2); e Catullo rammentando ad essi, quasi a loro sollievo, la propria delusione, finisce per mandare alla malora questi falsi protettori, questa genia di nobili amici, obbrobrio di Romolo e di Remo (3).

$$
* *
$$

Il libretto stesso catulliano contien la prova dell'amicizia, anzi della faruiliarità, ond'eran congiunti Catullo e Cinna. Istruttivo a tal proposito e il carme $\mathrm{X}$, un bozzetto a rapidi tocehi ed a vivi colori. Varo condusse un giorno Catullo a visitare la sua ganza, ragazza allegra e spiritosa. Cadde il discorso sulla Bitinia. La ragazza si

(1) Carme X, 9-13.

(2) Cfr. i carmi XXVIII e XlVII. I'isune e probabilmente L. Pisone Caesonimus, ̀̀ i due componimenti sono del 699/55; efr. Schwabe, Qnaestiones Catull. p. 251.

(3) Carme XXVIII, 13-15. 
aspettava il racconto di grandi ricchezze. Catullu la disinganno: non areva riportato di hi neppur tanto da comprarsi un meruento! Ma neppur la specialità del luogo, spli schiavi letticarii! E Catullo, chẹ si sente toco nella sua vanità di giovanotto raffinato del bel mondo, si lascia sfuggire una bugia: certo, per quanto le cose anlassern male, non andarno peri cosi a rompicollo, che egli non potesse procurarsene otto. Subito la ragazza a chioderli in prestito: essa voleva recarsi in portantina al tempio rli serapide! La buria areva aruto le gambe corte: Catulo subito la comfessa. Era stato Gajo Cinna, il suo sorlale. che li avera acquistati. Ma che importava? Era tutf uno: Catullo poteva usarne ensi liberamente, come se fossero suoi: "Ltor tam bene quam mihi pararim» iv. 32). I due amiei erano duncue in gionaliera consueturline, e probahimente compagni di arrenture scapestrate (1). Il carme fu seritto dope il ritormo di Catullo e di Cinna dalla Bitinia, e cioc nol 698/.iti. Cli sia il Varo (Varms mens, v. 1) di questo carme nom potrebbe dirsi con sicurezza. Se l'Altenu del calme XXX è Alfeno Varo (2), si protrebbe pensire appunto a quresto personaggio; altri (3) con magregiore probahilita, pare a noi, pensa a Quintilin Varn, amicn di (Mrazio, ehe ne pianse con commosie parole la morte nella XXIll ade del libro primo.

A Cimna e diretor anche l'epingramma CXlll li Catullo,

(1) Sodalis i. ehiamato da Catullo il ston Cinna, in X, 29, e, so ¿ giusto il supplemento sodalis, anche in XCV, 9.

(2) Ipotesi sui rapporti di Alfouo Varo con Catullo verli presso Kiessling, in Commontalionis in hommem Mommseni, 1. 354.

(3) Schiwabe, (luaest. Catull. 1. $289 \mathrm{kgg}$. 
una sanguinosa satira sulle disgrazie coningali di Pompeio. Mucilla è infatti nomignolo diminutivo, adoperato per significare IIncia, moglie di Pompeio: per designare le donne di facili costumi era comune l'uso del diminntivo (1). I due amanti di Mucia erano ora, al secondo consolato di Pompeio, crescinti a duemila.

Probabilmente mo di quei primi amanti era Cesare; come amante di Incia Io rammenta infatti Suetonio (Ces. 50). Lialtro era forse Mamura, che Catullo rappresenta nel carme LVII quale rivale di Cesare e compagno in pari tempo di sue sregnlatezze. Al ogni modo l'epigramma tende indirettamente a colpire anche Cesare. Cimna, se è l'Elvio Cinna scambiato ral popolo per uno dei congiurati, e rimasto vittima di questo errore popolare, era amico di Cesare, piros Kaioxpos, come dice Plutarco; ma ciò non potera certo sconsigliare Catullo dall' indirizzare all'amico un carme con una allusione ostile a Cesare. E possibile del resto che anche Cimna subisse il fascino del dittatore, come Catullo e Calon, e si convertisse allamicizia di lui, dopo essergli stato nemico. L'epigramma i rlatato dalla menzione del secondo consolato di Pompeio: 699/55.

$$
* *
$$

L'opera poetica piu famosa di Cimna fu la Zm!rmo, un poemetto certamente di imitazione alessandrina, sul genere del carme TXIV di Catullo. A tale poemetto si

(1) Cfr. i nomi contenuti nella lettera di Antonio anl Ottavio, che è riportata da Suetonio (Aug. 69). 
riferisce il carme $\mathrm{XCV}$ di Catullo, che noi in seguito esamineremo. Ci rapporta Catullo che la Zom!lrma fu pubblicata dal sun autore dopo nove anni da che egli vi avea pristo maun (r. 1-2) :

Znỵrna mei Cinnie nonam post deniqque messem, Ginam coepta est, nonamque edita post hiemom.

E la motizia fu tradizionale presson i dotti romani (1): anzi poiche Catullo diee: mmum post messom e monam post hiemem, qualche wrammatico intese che Cinna vi laromase attorno dieci anni (2) $)$.

Fu ritenuto dai dotti dell antichita e si ritiene generalmente ogri che l'esempio di Cimna sugrerisse ad Orazio il suo famoso consiglio ai poeti, di porre sotto il pressoio sino al nono anno le loro opere (3). A dir vero non sembra verosimile che (Mazio sia ricorso proprio a Cimma per apportare un esempio di perfezione poetica. Orazio, come i noto, parla con tono superiore di Catullo e di Calvo, e lilegrgia un ammiratore dei due poeti (t): non

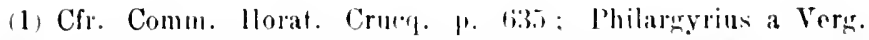

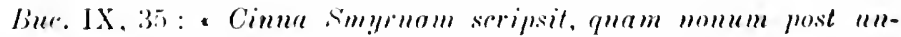
num, ut Cutullus ait, stidit, id yuod ot tuintilianus ait w. II lungo di Quintiliann is X. 4, 4. Porfirione, ad Art. Poet. 388 (r. piu sotto).

(2) Servio a Bur. IX, 35: "Cimma... qui seripsit Simyrnam, quem libelluem derem anmis rlimarit,.

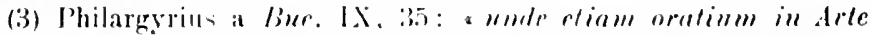

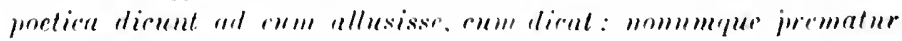
ill anulum n.

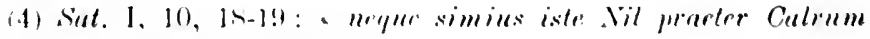
et doctus cunture cintullums. 
si comprenderebbe darrero che egli riserbasse a Cimna un trattamento di ammirazione: tanto più che non par dubbio che il poemetto di Cinna non avesse pregi di nitore e chiarezza (1).

È bensì vero che il Weichert (2) interpretava a questa maniera il pensiero oraziano, che Orazio approvasse cioi la diligenza di Cinna, ma non ne approrasse il risultato, cioè il poemetto! Ma è un po' troppo, mi pare: e meglio, in ogni caso, sarebbe ammettere con un antico critico (3) che Orazio'si rolesse far ginoco di Cimma, che areva sciupato nove anni in una inutile fatica.

Probabilmente il riscontro è affatto casuale. Anche, se ben si guardi, i dati non corrispondono. Dal passo di Catullo può risultare solo che Cimna lavorò nove anni attorno alla Zmyma (4); nel passo dell'Arte poetica oraziana non si consiglia un lavoro di lima che duri nove anni: vi i detto invece che il poeta, composta l'opera sua, debba metterla a giacere fino al nouo anno,

(1) Philargyr. 1. c.: " fuit autem liber obscurus adeo ut nommulli eius aetatis grammatici in eum scripserint, magnamque ex: eins enarratione sint gloriam consecuti. Quod obscurus fuerit etiam. Martialis ostendit in illo versu: Non lectore tuis opus est, sed Apolline libris. Iudice te maior Cinna Mrorone fuit . (Marziale $\mathrm{X}, 21)$.

(2) Weichert, De C. Helvio Cima poeta p. 174, nota 15: ".... cuius curam ot diligentiam dum lando, ipsum opns $\mathrm{nom}$ admiror ecc. 3.

(3) H. Boschius in Observ. et Yot. in Anthol. Araec. p. 152 seg., presso Weichert, 1. «.

(4) Dico 'lacori, perchè Catullo dice che la Zmyrna fu edita dopo nove anni quam coepla est, non già dopo nove anni la rhe fu tompiuta. 
griachè allora solo potrì decidere se egli debba pubblicarla o distruggerla (1).

I nove anni di Cinna somo contrapposti all' unico ammo di Ortensio. Il terzo versu infatti è Millia cum interea qningenta Hortensins moo... Il quarto verso manca. Il qnimgentu millia (cui dovera seguire un genitivo) si riferisce certo al concetto di 'versi', ed ì naturalmente numero di amplificazione iperbolica (2); l'uno si rifirisce al concetto di amno. Benchè questi due fatti sieno sieuri, pure non è sicuro quale concetto fosse contenuto nel verso quarto.

Parrebbe orvio che allindustriosa cura di Cinna, nel curare per nove amni la Smirna fosse contrapposta la negligenza facilona di Ortensio, nello schiccherare in un inno continala di migliaia di versi. Inti i supplementi (3):

(1) Arte poet. 386 sgg.:

niøguisl tame.l olim

Soripserin. in Marei desiendat indicis anres

Et putris ef nustras, nommunte promatur in ammum

Membranis intus positis : Mellere liserbit

Qund num minleris: newrit vox missn reverti.

Notevole is l'interpretazione di Portirione: Exemplo Cinnae poterit uti, ut carmen summ, si malum est, norem annis dissimmlet .. Il Friedrich ediz. li Colull. 1. S19 eschama: "Iortref-

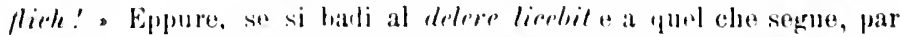
proprio edse Orazio parta lal presupposto di una brutta poesia, th 11n malım" rarmen".

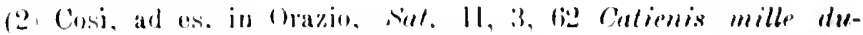
centis is manifestamente sehermo iperbolico.

(3) V. varie concetture in Thumas, Les pormes de calulle...

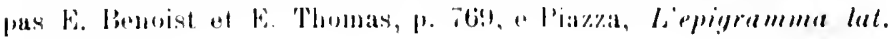
1. $168-173$. 
In pede stans fixo carmina ructat hians (Partenio), Aut plura anno se scribere posse putat (Pascoli), Perscribens anno carpat ineptus eam (Friedrich). Senonchè il riferimento di questa punta satirica contro Ortensio ha suscitato difficoltì non poche. Ammettendo che l'Ortensio poeta sia il famoso oratore, esso sarebbe l'Ortalo, a cui si rirolge con sì teneri accenti Catullo nel carme LXV; par difficile che qui abbia per lui così pungente sarcasmo (1). Inoltre, ben si comprende come in 111 epigramma si contrapponga il piccolo poemetto di Cinna ai ponderosi annali di Tolusio; ma un po' meno si comprende che si cominci a contrapporre Cinua ad Ortensio, e poi questo sia lasciato da parte e per tutto il resto si parli di Volusio. Coloro che furono vinti da cotali difficoltà prescelsero due vie: o immaginare m supplemento che togliesse l' idea del contrapposto tra Cinna el Ortensio, o mutare addirittura il nome Ortensio. Son della prima specie i supplementi (Iortensins uno) Mivetur Volusi carmina facta die (Peiper), Anno a me versum flagitat increpitans (Gandiglio) (2), Mense levis quot habet carte legit Volusi. E' facile ad ogni modo osservare che anche così il povero Ortensio non fa la migliore delle figure; nè quest'uscita di Catullo potrebbe certo interpretarsi come uno scherzo benevolo el affettuoso; specialmente

(1) Questo aromento fu svolto dal Gandiglio, Il earme $\mathrm{XCl}^{\top}$, li Catullo, Adquilat, 1903), p. 6. - Non par lacile ammettere col Menozzi (Je Catmlli Carm. XLIX et LXXXXI, Trani, 1895 , p. 25-26) che Catullo abbia voluto dare un amichevole consiglio ad Ortensio di segune l'esempio di Cinna.

(2) Versum per versıtım. Il Gandiglio propone altri tre modi per esprinere a un dipresso to stesso pensiero (op, rit. p. 15). 
per la forma, che non è di allocuzione diretta allamico, cume si aspetterebbe nel caso di ma celia giocosa. Altri mutano addirittura il nome Hortensins: horrentins nllo Pleituer: Tamusims uno Versicmlormm anmo quolibet ediderit, Froehlich: Hatrianus in mo Versicnlornm anno putidns eromnit Munro: T'anusins ed Hatriums (= di Hatria) equivarrebbero a Volusins.

Io ritengo probabile che il carme XCV risulti di due epigrammi : il primo sino al v. 4 perduto: il secondo dal r. 5 al 10. Tutta questa parte dei carmi catulliani, dal carme LXXXI sino alla fine, è scritta nei codici senza alcun interstizio: la divisione in carmi è opera degli editori moderni. I due epigrammi furono messi l' uno dopo l'altro, perche trattavano In stesso soggetto, la Smima di Cimna: così l'un dopo l'altro furono messi i carmi lall'LXXXVIII al XCI, perche trattavan tutti di Gellio. Nel primo epigramma (v. 1-4) il contrapposto era tra Cima ed Ortensio; nel secombu (v. 5-10) tra Cimna e Volusio (Tanusio).

Non credo poi che l'Hortensins del nostro epigramma sia da identificare con l'()italus del carme LXV, nò veggo che di tale identificazione si sia apportato alcun argomento, non potendo dirsi tale il fatto che l'oratore famoso ci chiamasse O. Hortensins (1)talus (1). E neppure si ha prova che ii poeta IIortensius fosse proprio l'oratore. il che noi quindi nom pessiamn in modo sicuro confermare (2) : pur ritencendo prohabile l'identificazione.

(1) Propriamente IIortalus. V. supra (). Ilortensius IIortalus

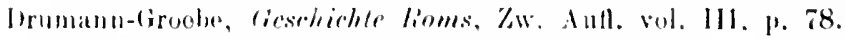

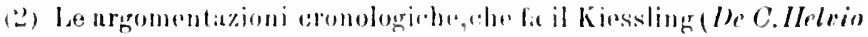

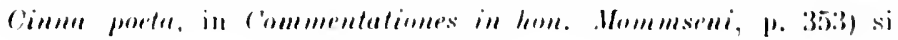


Velleio Patercolo (II, 16, 3) cita anche gli Annales di Ortensio. Il titolo potrebbe far pensare ad un poema epico, come quelli, di pari nome, di Ennio, Accio, Furio, Tanusio; ma Velleio sembra voler citare la testimonianza di uno storico, sarà quindi da pensare ad un'opera in prosa? Si tratterì sempre dello stesso Ortensio? Lo ignoriamo. Quel che non è dubbio è che all'epoca di Catulio vi era un Ortensio, poeta di carmi erotici. Plinio (epist. $\mathrm{V}, 3,5)$ cita il suo nome tra quelli dei poeti licenziosi, e vuole con quella lista di poeti giustificare le proprie marachelle poetiche. Non altrimenti Ovidio (Trist. II, 441) "Nec minus Hortensi, nee sunt minus improba Servi Carmina ». Gellio riferisce sui suoi carmi un giudizio non farorevole (XIX, 9, 7 ): "Laevins implicata et Hortensins invenusta et Cinna inlepida et Memmins dura ac dinceps omnes rudia fecerunt atque absona». Eे vero però che questo giudizio non è di Gellio, bensì di alcuni Graeculi, che esprimevano al retore Giuliano i loro giudizii sui poeti romani; Giuliano, per disingannarli, non apporta versi di Levio o di Ortensio o di Cimna o di Menmio, bensì di Porcio Licino, di Lutazio Catulo o di

ondano apjunto su tale identificazione; ma esse per molte ragioni, somo affatto incerte. Dice il Kiessling che Catullo dedico ad Ortensio (identificato quindi con l'Ortalo del carme LXV, 2) la Chioma di Berenice nel 695 o 696 ; poi dopo il ritorno dalla Bitinia comincio ad ammirare ed amare Calvo, ed edotto da lni cominciò a giudicare severamente de Asiamo dicendi genere quo Hortensius ntebatur ». Ma ammesso che Ortensio scombiceheratore di versi sia il grande oratore. che cosa abbia che fare il genere asiatico della sua oratoria coi cinquecentomila versi scombiccherati da lui in un anno non si rieste a comprendere: Catullo per colpire il genere oratorio ne avrebie assalito i tentativi poetici?

C. Pascal 
Valerio Edituo! Ad ogni molo nel giudizio ali quei Graeculi sono accomunati nella meslesima condamma Ortensio a Cinna, che invece Catullo pune l’umo di fronte allaltro. Poco oltre diellio (XIX, 13, 5) citando due versi di Cimna lo chiama "non ignolsilis népne indoctus poeta " (1).

$$
* *
$$

Questo epillio di Cinna í chiamato da C'atullo, nel carme appunto che gli i dedicato (XCV) paren monumenta, e per la sua piccolezza í contrappensto alle migliaia di rersi di Ortensio, anli amali di Volusio, il tumido Antimaco di Padova (2). La poesia alessimuldina aveva apportate il gusto, che rimase joi trionfatore nelle varie epoche della letteratura rmana, delle piceole imprese portiche, wei hrevi componimenti, wo la legenenda mitologica era la materia dellat trattatzione. ma i cauratteri di

(1) Il significato di indortus detto di poeti. is la indurre dal signiliato di dortus poetu. espressione della puate trattiamo a propusito di Calvo; cfr. p. 10-14.

(2) Carme $\mathrm{XCV}, 9-10$ :

Prarsa mes milhi sint cordi mommenla smblalis, .1t popmlus tumidn gatuleast Antimurluo.

Simlalis manca nel corlice Sangermante Parig. 141:37) o nell'Oxonionse (Bib). Berdleiana, lihr. ran. 30 : ir nellat plizione aldina del

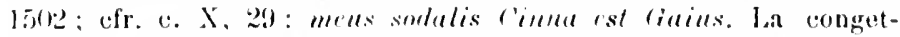

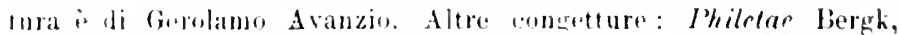

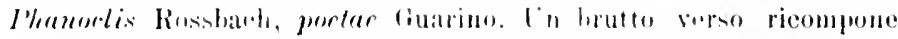
i) Barlusens: Proren mei mithi sinl C'imme rordi monumenta. Un nono li preta green contruppesto anl Antimace darebbe buon

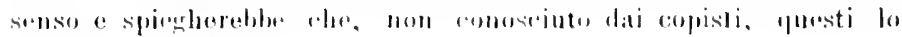
aressern olmessus. 
essa erano essenzialmente erotici e sentimentali. La storia delle passioni infelici fornira infinita varietà di toni, e dava modo al poeta di mettere in mostra tutta la slla virtuosità elegiaca. La trama più semplice della leggenda primitiva si andara ampliando e complicando con fusioni ed intrecei di altı leggende, e con sviluppi di particolari leggendarii: questo dara modo al poeta di rendere qua e là più vivace l'azione, e di rappresentare i personaggi con determinazioni e con tratti, che lichiamarano quasi al pensiero la scena drammatica. Le grandi intraprese epiche, i lunghi poemi doverano essere ormai abbandonati. Uno dei duci e modelli di questi nuori indirizzi li poesia arera detto la sentenza: "grau libro, gran danno!,. (1). I poeti quindi potevano ormai mirare a più umile segno.

E solo così poterano sperare di conquistare la gloria. Ed infatti, dice Catullo, la Zmyrna giungerì sino alle spumandi onde del Satraco, e cioè sino ai luoghi stessi ov'è nata la leggenda di Zmỵrna (2); mentre gli annali di Tolusio morranno presso la stessa Padua, una delle due bocche del Po (3), presso cui sono stati composti. E evidente che al Satraco, famoso nelle leggende dei poeti si vuol contrapporre loscura riviera, ove sono nati e dil cui non spiccheranno alcun rolo gli Annali di Volusio.

(1) Cfr. Callimaco presso Ateneo, III, 1 (Schneider, fr. 3507) :

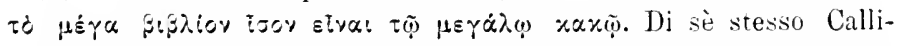

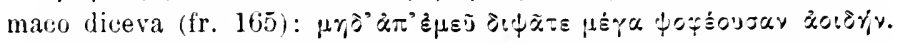

(2) Che la leggenda di Zmyrna o Myrrha forse localizzata nella tradizjone sulle rive del Satraco, si puo dedurre forse dall'Etymologicum Waynum p. 117, 37; Nonno, Jion. 13,458; Tzetze a Licofr. 448.

(3) Polibin II, 16; 12: Hasia. 
Ma chi i questo Volusio, il cui carme pesante, lungo a tumido viene contrapposto al piccolo monumento di Cimna: A Volusio ed ai suoi anmali si riferisce anche un altro carme catulliano. il XXXVt, nel quale è contenuta due volte una espressione hrutalmente senncia, Amuales Volusi, curatr carta (1).

Lesbia avera votato a Venere ed a Cupidine che, se si fosse rappattumata con Catullo, arrebbe lammato al fuoco i ressi di un pessimso poeta, el il poeta annumzia la triste sorte agli annali di Volusio. pleni raris of inficetiar'll'!.

Di uno serittore che aresse nome Irolusins non si ha da aleuna altra funte notizia: ma i naturale che l'attenzione si fissasse sopra un passo delle epistole di Seneca, nel quale si parla di Ammles Tumusii come di opera poetica. Eeco il passo (E). XClll, 11): "st paucorum rersumm liber est et quidem landamdus atyne utilis: ammales Tannsii sciss quam ponderosi sint et quid ro-

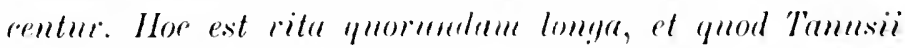
sequitur anumles ». Anche in questo passo ì contrappostu un carme di pochi rersi, liber landandus atque nti-

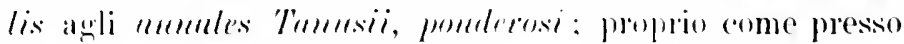
Catulln il piecolo liber ali C'mma andi ammules Tolnsi.

Ma ro ancora di pia. Seneca sembra volersi proprio rifirige ai dur carmi catulliani mej quali è menzionato

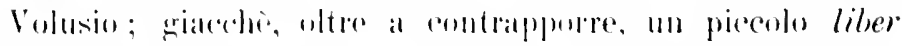
pretion ai pouderosi annali, comme fit catullo nel car-

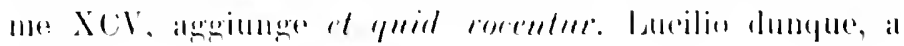

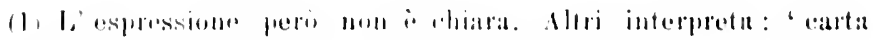

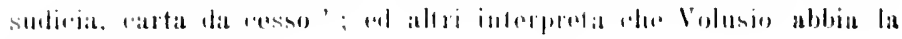
diarrea dei versi. 
cui Seneca scrive, sa come ermo chiamati gli annales: come? evidentemente è un' allusione al cacata carta del carme XXXVI: il brutale dileggio era diventato tradizionale e proverhiale. E quindi opinione di ralenti critici che Tolusio sia proprio Tanusio (1). Io credo sia da accettare tale identificazione. Sarebbe già un fatto molto strano che contemporaneamente si avessero due poeti, Volusins e Tannsins, e che entrambi avessero scritto un poema ponderoso intitolato Annales. E si aggiunge che le parnie di Seneca paion proprio riferirsi ai versi catulliani; sris... quid rorentur è una espressione di riserbo e di discrezione, che sembra appunto alludere arl una frase sconcia, che ripurni di ripetere, ma che sia diventata famosia.

Nel carme XCT si contrappone dunque il piccolo ed elegante poemetto di Cinna al pesante poema epico di Tanusio. Questo autore è citato altrove come scrittore di storie (2); ma forse egli si illuse di conquistare l'immortalità con un grande poema epico sulla storia di Roma, al quale rette il titolo di Ammales, sià usufruito per i loro poemi da Enmio, Accio, Furio. Pel qual ragione Catullo lo chiami, invece di Tamusins, Tolusins, noi nou sapremmo dire. Noi comprendiamo lo scherzo catulliano,

(1) Nego l'ilentita P. E. Sonmenburg, Der historiker Trmusius Geminus und die Annales Tolusi. Bomn, 18S2. La sostennero to Haupt, Quaestcones Catullianae, 1837, p. 98, lo Schwabe, Jahrb. Philol. 1884, p. $380 \mathrm{sgg.;} \mathrm{il} \mathrm{Friedrich.} \mathrm{Catull.} \mathrm{1.} \mathrm{520-1,} \mathrm{el} \mathrm{altri.}$ $\mathrm{V}$. sulla questione anche Ellis, Academy. 12 Margio 1883, e Niese, hh. Mus. XXXVIII (1883), p. 600.

2) Suetonio, Caesar 9: " meminevunt huius coniurationis Tanusius Gemimus in historire ecc. ; Plutarco, Ces. 22 : Tavij-

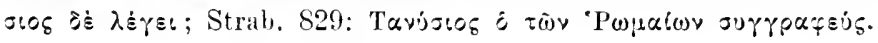


quando per tesignare Mamurra adopera il nomiznolo. Montula: ma nel nostro caso la punta ei sfurgue. Le ipotesi presentate a tal proposito dal Friedrich (1) non ci soldisfano punto. Se Catullo aresse storpiato il nome di Tamnsius: in Tolusius solo perchò nel verso precedente ¿' un percoluent. o perchè volvere è adoperato. quando si tratti di agonizzanti, in un senso che molto si arvi-

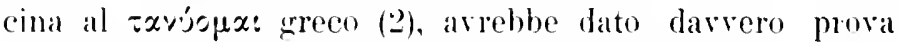
li proco spirito, ed arrebhe fatto uno scherzu vano, perchi da nessun compreso, e in ogni caso non tale che mettesise conto di comprendere. Questi seherzi allora riescono, quando ben si attagrliano e denotane con immeliata evidenza la persona. Si agriunga che nè l’uno né ladtro di questi supposti gruochi di parole conterrebbe alcuma punta satirica contro Tanusio; ed il primo anzi, furllo dellaceenno alla parola perrolnent che precede, nun potrebhe certo valere per il carme XXXVI, ove il nome Volusi ò nel primo verso, e non è preceduto da nua forma di rolvere, sicchi lo scherzo non riescirebhe intelligihile. To credo preferibile supporte che Volusius fisse un nomignolo, scherzosin e popolare, di Tanusins, cosi come credo probahile clur Mentmla forse il nomimonlo popolare e soldatesco di llammra. Niturabmente

(1) Colulli Veronensis liber, p. 50(1-5)21.

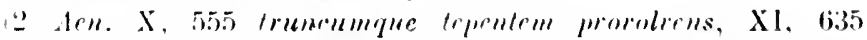

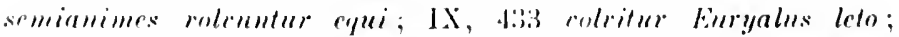

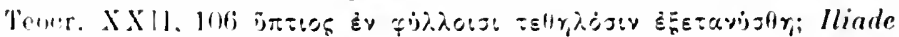

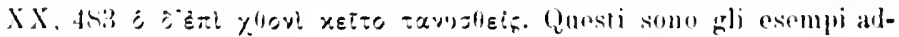

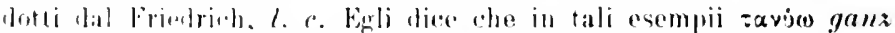
gleish yrhenumh wird rie volvo. Ma altro is il distendere le memhra brll' interidimento della morte. altro i il voltolarsi sulla terra morli sprasmi loll' agonia! 
rimane sempre ignota l'aliusione di tal nomignolo popolare: non si rarvisa cioè a qual difetto o capriccio o debolezza di Tanusio volessero riferirsi i suoi amici o i suoi nemici, col deformarne il nome in Volusio.

Tanusio-Volusio è il " tumido Antimaco , nominato nelI'ultimo distico di questo carme XCV. Il distico cosi strona:

Parva mi mibi siat cordi monumenta...

At populus tumido gaudeat Antimacho.

Come si vedo, dal penultimo verso é caduta l'ultuma parola. Abhiamo sopra rammentato i rarii supplementi proposti. Comunque sia, in questo disprezzo di Antimaco Catullo rimane ferlele al giudizio di un suo grande modello, di Callimaco, il suo Battiade. Abbiamo già visto come Callimaco guardasse con disprezzo le grandi iutraprese poetiche, e sentenziasse: "grosso libro, grosso dauno!,, Ed appunto a proposito di Antimaco, Callimaco griudicava la sua Lide un poema grossolano e non chiaro (1): ed a chi gli obbiettara che Antimaco aveva avuto lapprovazione di Platone rispondera riconoscendo a Platone il gran "alore filosofico, na negandogli il diritto di giudicare i poeti (2). Naturalmente roveva essere proprio questo il giudizio del poeta reronese, che celebravi le piccole bazzecole, diventate, per virtì del genio poetico, monumenti letterarii. Per opera sha e dei poeti della slla

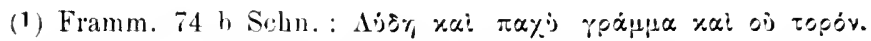

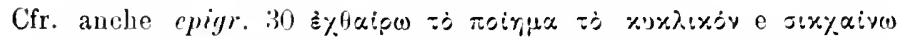

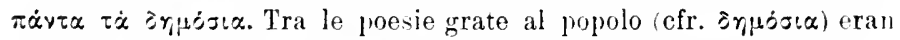
quelle di Antimaco (efr. populus tumirlo gaudeat Antimacho).

(2) Cfr. Proclo, a Platone Tim., I, p. 28 c. 
scmola, "les Romains pourront desormais se convainere, en les lisant, qu' il n’y a pas de grands et de petits sujets: tous solit grands pour un vrai pocte comme lui, tous sont petits pour un manvais:

In tenui labor: at tenuis non gloria, si quem

Numina laeva sinunt anditgue vocatus Apollo 2 (1.

$$
* * *
$$

I critien dei quali fiellio (XIX, 9) riferisce il grimlizio trovavaun imlepida, e cior sunza spirito e senza grazia i carmi li Cinna. Cili antichi trovanno pure che la Smirna repa pusia molto oscura of areva hisogno di commenti. ( $\because$ ne informa tuno seoliaste di Vererilio (2): "suctonio rita Iunn di tali commenti, quello del rrammation Crassiejo, "riporta su lui e sull opera sua un wrazioso epiyrammal :

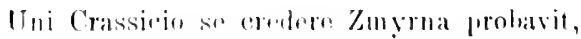

Desinite imfocti condugio hane petoro!

Soli Crassicio se dixit mubre velle.

Intima eni soli nota sua extiterint (3).

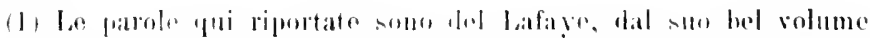

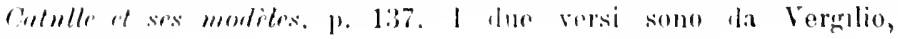
Ifu. IV, (;-7.

(2) Filargirio a Verer. erl. IX, 35: e fuit autem liber obscurus

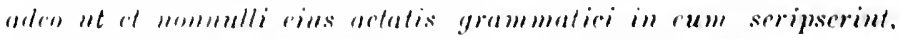

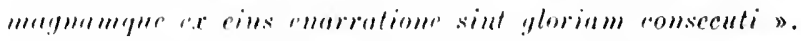

(3) Suctoniu, lle yramm. 18: * L. Crassicius... commentario

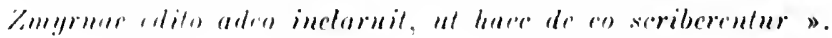


Marziale trasse argomento dalla famosa oscurita della Smirna per qualche suo piacevole scherzo:

Non lectore tuis opus est, sed Aprolline lihris:

Iudice te maior Cinna Varone fuit.

$$
\text { (X, 21,3). }
$$

Versiculos in the narratur scrihere Cinna.

Non seribit cuius carmina nemo legrit.

$$
\text { (III, 9). }
$$

Cinna nel secondo epigramma í quasi appellazione antonomastica, per desiguare poeta, che per la sua oscurità non si fa leggere. E si che Catullo aveva vaticinato che i pii remoti secoli to leggerebhero! Zmygruam sana din saecula permoluent.

Ad ngni modo è fuor di dubbio che questi grudizii sfavorevoli erano esagerati e troppo sereri. A noi bastano le insigni testimonianze di Catullo, di Vergilio, di Gellio per credere al valore poetion di Cinnd. Se Catullo gli prediceva l'immortalità o gindicava che i suoi carmi erano piccoli monumenti (1), se Vergilio sotto pastorali spogrlie asseriva non potere cantare cosil degna di Varo " di Cinna (2), se Crellio con espressione non certo di ammirazione fervida, ma pur di sincera stima, il chiamava non ignohilis neque indoctus poetn (3), questi apprezzamenti provengono da persone di cosi fine gnisto e di cosi alto giudizio, che non è possibile disconoscerne o attenuarme il valore.

(1) Carme XCI.

(2) Ecl. IX, 35,

(3) Gellio XIX, 13, う. 
E r’o anche mon'alta insigne testimonianza: due bei distici del preta Valyio. Essi riguardano Codro, mo pseudonimo forst. ele nasoonde non si sa qual nome di poeta, quel Codro stesso, i cui meriti poetici somo con così alto elogio esaltati nellegloca VII rergiliana, ove il pastore Coridone annumzia che Codro per i rersi suoi si arvicina a Febo (1). Diceva dunque Valgio in una egroga (2), nella quale certamente dovera essere rappresentata una zara di canto, che Codno cantava conl lammoniosa roce di Cimna i versi di Cimna stesso: nè mai roce più soave era rifluita dalla bocea di Nestore o di Demorducu, il cieco canture dei Feaci :

Corlrusque ille canit quali tu roce canchas,

Atyue solet numeros dicere, Cinna. tuss;

Duleior ut numyuam Pylio profluxerit no

Nestoris ant doeto peretere bemodeni.

(1) Whe VII, 21:

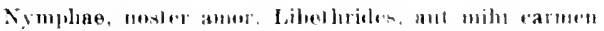

Qunle men rowlow coucedite - prosima l'hoeli

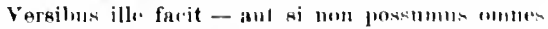

llic nrguta sacra pendelpit tistula pimu.

Al complimento di Coridone a Corle rispoude Tirsi insolesntemente, angurandesi la coronis di wha nel ertame poetion. sinehe Codro ne crepi di invidia. Fd a certani poetini di Codro si riferisee anche l' iurgia Corlri dell' egl. V.

(2) Il passo is presso lo Sonl. Veronese a Vera. orl. VII, 22. e

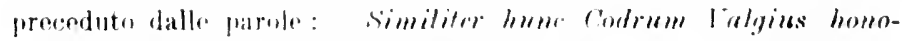
rifiep apprlat. ef yuatem in erlegy de en ait... . A questo medesimo passo servio aunota: Codrus poeto einsdem temporis fuit, ut lalgius an reglis suis refent ": sarit in questo pusso da correggere in erlogis, olpurre nel primo qundam in slegia. 
É una lode insieme e per Codro e per Cinna.

La Zmyrna di Cinna trattava certamente il mito di Smirna o Mirra, che, per opera di V'enere irata, fu accesa di abbominando amore per il padre suo Cinpra, e si congiunse con lui ignaro. Conosciuto il misfatto, il padre perseguì la figlia, che andò fuggendo atterrita, e si ridusse infine in una selva, ove fu mutata nell'albero del suo nome. Ia dal nefando concubito era nato ddone (1). È l'argomento trattato da Ovidio nel libro X delle Metamorfosi (r. 298-502), non senza molte proteste contro l'orrore del misfatto (r. 300-318) (2). In Grecia era stata trattata da Paniasi (3) e da Nicandro (4) : da quest'ultimo, probabilmente mediante in contaminazione con altre fonti, e con molta libertà di elaborazione personale deriva la trattazione ovidiana. In questa ò specialmente uoterole lo sviluppo dato alla rappresentazione della sciagurata passione di Mirra (Smirna), e certamente, come fu già notato (5), non per un perrertimento dei sensi, ma per una ricerca di condizioni psicologiche strane. Si può essere sicuri che a questa parte dava largo sviluppo anche Cinna : ciò era infatti nell indole della poesia catulliana e dei poeti catulliani, così come era stato dei poeti alessandrini. Forse anche Nicandro fu, in parte almeno, mo-

(1) Cfr. Igiro, Fab. 5s; Antonino Liherale. cap. 3.1: Apollodoro III, 14, 4 ; Scol. a Teocr. I, 106 ; Servio a Verr. erl. X. Is ed Aen. Y, 72.

(2) Sopra la trattazione ovidiana della favola cfr. G. Lafaye. Les Métamorphoses dOcide, p. 171-2, e Castightioni, stutii intormo alle fonti e alla composiaione delle .letamorfosi di Oridio, p. (is..

(3) Cfr. A pollodoro III, 183.

(4) Cfr. Antonino Liberale, 34.

(5) Lafaye, op. eit. p. 172. 
dello a Cinna: $i$ da credere altresi che non poca erudizione mitologriea erli ponesse nei versi snoi, se ad intenderli occorrevano le dichiarazioni di Crassicio: il mito di IIrra del resto vi si prestava, si per alemi particolari, sì per la rarietà dei nomi del padre e della figlia, si per le diverse localizzazioni, che esso ebbe, nelle tradizioni poetiche dei Freci.

\section{***}

Il primo dei frammenti della Zmyma i conservato da

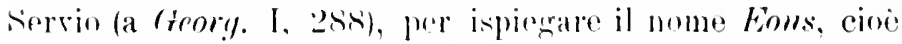
Lucifero. Esso e:

fr. I. Te matutinus flentem conspexit Eous,

Et flentem fraullo vidit post Hesperus irfem.

Hesperms idrm i Hesperms qui illem est atyme Eams: Gima i un poeta olscmms! Pii chiaro e quel medesimo idem, drette per il medesimo Itesperes in catullo (LXIl :34): "Norte lalemt fares. quos iltem safpe revertems, Hespere. mutato compremlis momime cosslem. Nel frammente di Cimna si tratta certamente della seiagurata assillante passione di simirna: essa me zeme i gromi e le notti. Chi sia che parli non sappiamo: i probabile, che il poeta stesso, interrompendo la forma della narrazione si rirolera eon questa speeje di allocuzione alla sua eroina. Del resto il pensiem rontennto nei due versi è frequente nei preti antichi. Vedi sia nell' Meliale di Calli-

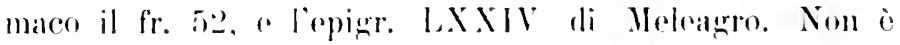
sicuro che a reminisernza di Cinna a di Catullo, pinttostoche a propria ispirazione siano da assegnare Vergilio (Geory. IV, fliti): "Te remiemle die, te deeedente canebat" 
el Ovidio (Met. V. 440) "Illam non milis veniens Aurora capillis Cessantem vidit, non Hespems ». Ma direttamente da Cinna discende l'autore del Carmen de rosis (v. 45-46): "Qnam modo nascentem mutilus conspexit Eous, Hanr rediens sero respere vidit anmm ». Il notivo poetico ebbe del resto larga eco anche presso Seneca (1).

L'altro verso che rimane della Zmmona is conservato da Prisciano, else per documentare la voce alens masc. cita da quel poemetto (2) :

fr. II. At scelus incesto Cinyrae crescebat in atvo.

I codici hamno, per Cinyrae, Cinnce o Cirence: trattandosi di un nome difficile e poco noto è naturale la colruzione. Cimyrae è emendazione già antica, invece della quale altri ripose Zmurnue, Zmyrnae. Zmyrnae in alvo ha senso chiarissimo; meno orvio è scelus Cinyrae; il 'misfatto di Cinira', s'intende, involontario. Cinna ì poeta obscurus; e del resto la lezione Cinyrae è più vicina alle lezioni Cimnae, Cirenae dei codici (3).

Queste somo le uniche reliquie dei versi del famoso poemetto, oltre una citazione di mua sola paroha, tabis,

(1) Tieste 613; Ippolito 648; Edipo 741. Cfr. anche Stazio. Tebaide VJ, 388.

12) Prisciano VI, 16, 84, p. 718 P. : "Cima in Smyma ece. . Carisio, I, p. 61 l'. $80 \mathrm{~K}$.. scorrettamente: incertum turpi.

(3) Il Voss traduceva: "Aber des Cinyras Griiuel erwuchs im geschiondeten siohosse. Incesto ì naturalmente nel signifionto etinulogico, in opposizione a castus, come in Lucr. 1, 98; livin $\mathrm{I}, 45,6$. 
che Cimnar secondo il stammation Carisin arrebbe per primo adluperatn (fr. III) (1).

$$
* *
$$

P'ieculu, ma moterole doenmentu della poesia di Cinna

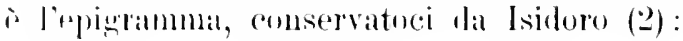

lir. IV. Haes tili Areteis multum invigilata lucernis

Carmina, quis ignes novimus aerios,

levis in aridulo malvae descripta likello

Prusian vexi munera navicula (3).

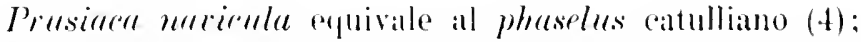
r prosiarere is da Prusial le di Bitinia: il poeta ha dun. que portato ens sè dalla Bitinia $i$ ealmi rigilati alle lucerne aratie, per farne dumo ad an amico. Cle cosa somo yuesti carmi? Inciano Miiller pensi che si trattatse proprin di un estemplare dei armi di Arato (5). () si

(1) Carisio, 1. 93 K.: * Cinma antem in Zm!nmu huius tubis divit mullo auctor". - p. $145 \mathrm{~K}$.: "huins tabis Cimna in \%myrna dixit, mullo ante se usus aurlorer

(2) Isidoro, Oriy. VI, 12 (a proposito dell' uso degli antichi di sirivere molle foglie di malva).

(3) l'er quanto riguarda la lozjome. ansorviamo nel v. 1 Arreis doi curlici (non Arateis): $\checkmark$. sotto : e la forma inrigilala ; i co-

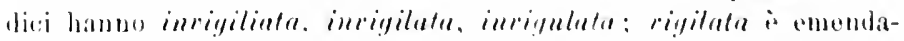

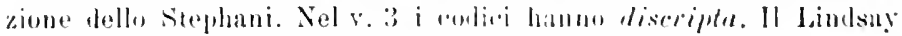

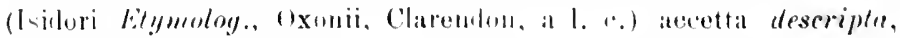
ma renserva imrigilate.

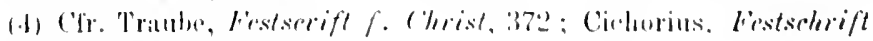
f. Hirselefold, 467.

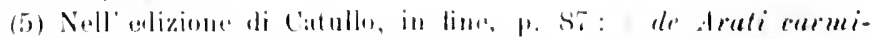

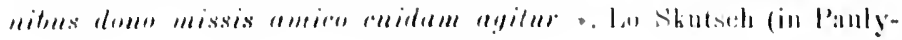


tratterì invece di un carme di Cinna, di soggetto astronomico, modellato sulla imitazione di irato? È difficile avere la certezza assoluta per l'una interpretazione o per' laaltra. Crovenale ha $(\mathrm{I}, 51)$ "haer ego non credum Vemusina digna lucerna? » e cioè "non dorrei credere questi soggetti degni di satire ad imitazione delle orazioni? » Di qui dunque si arvalorerebbe l'interpretazione di carmi imitati. Il imltmm invigilata indicherebbe il lungo sturio ed il grande amore dell' imitatore sui carmi di Arato (1). E che siguificato arrà poi il quis iynes novimus aerios? Lasciamo da parte la congettura movimms nel senso di decantarimns (Pithon, Barth, Weichert), che non è giustificata.

Il quis, cioè quibns, sembra naturalmente essere riferito a carmina, pintostoche ad Areteis lucernis. Es se si riferisce a carminu, il significato di tutto l'epigramma è di una trascrizione dei carmi di Arato: giacchè il significato è che da quei carmi (non certo dai proprii!) il poeta ha appreso la scienza degli astri (ignes aerios, cioè sideru). Ma potrebbe anche essere che il quis, con di-

Wissowa, Realenliylilop. sotto voce Helvins, 12) non si decide: "Gewölnlich denkt man an ein Exemplar oder eine Uebersetzung der $\Phi a$ crojeva Arats. Die Bedenken hiergegen hat Traube (Festschrift f. Christ, 372 s.) ausgesprochen, der selbst an Technopaegnien denlit.

(1) Cfr. Ciris 46: "Accine dona meo multum rigilata labor""; Stazio, Sitvae, IV, 6, 25: "docto multum rigilata Hyjomi", Theb. in fine: "O mihi bis senos multum vigilata per annos s; Ovidio, Fusti IV, 109: "vigilatum carmen"; Giovenale VII, 27: "vigilata carmina . Il multum vigilata della Ciris nella medesima collocazione metrica è imitazione da Cinna; quello di Stazio può essere da Cinna, ma credo più probabile che sia dalla Ciris ; efr. Ganzeanüller, Jahrb. f. I'hilol. (Supplem. XX, 365). 
sposizione un po' libera, si riferisse ad Areteis lncemis; a tornerebbe allora molto bene il senso, che per lo studio li Arato egli aresse appreso le cose celesti. Non bisogna limenticare che Cimma i poeta obsmmes: e che coefficienti dell'oscurità sono appunto tutti questi sensi ambigui ed incerti. Del resto il passo di Cimna i imitazione di un passo di Callimaco, ove pur si fa menzione delle ve-

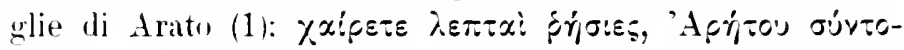
vos dyessvin. Qui non par dubbio che queste protes sieno proprio i carmi di Arato (2), e riteniamo quindi pii probabile che in tal senso sia da interpretare altresi l'epigramma di Cinma. Questi non mandó forse all'amico un suo carme ad imitazione dei Phaenomena, ma gli portì un esemplare di quel poema, traseritto sopra foglie di malva.

$$
* *
$$

Altro componimento poeticu di Cinna fu il Propempticon Pollionis. Il titolo di í conservato dal Carisio, che no cita quattro versi (3):

fr. $V$. Nec tam donorum ingenteis mirabere acervos Innumerabilibus congestos unlique sacelis. lam inde a Belidis natalique urbis ab annu Cecropis atyue alta Tyrii iam al, origine Cadmi.

(1) Callimato. Elvigr. 28 (27 Wil.h. La forma ionina Aproo adoperata da Callimaco a comsiglia a eonservate nell epigramma di Cinna la forma Arrteis (mon Arateis) del codice (inclforbitano o de.l Monacense.

(2) Altrinenti sarehbe se si aveste a lewere. secondo un' anticn

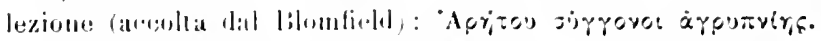

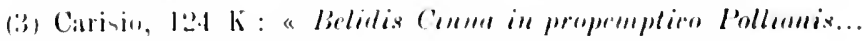

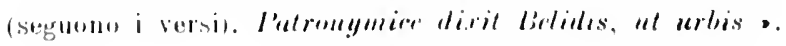


Il pensiero evidentemente è interrotto: il frammento non è completo: al nec tam doveva corrispondere un quam: il Kiessling (1) suppose che il pensiero dell'apodosi fosse: "quam in philosophorum scholis sapientiae et eloquentiae operam daturus. es ».

Che i rersi si riferiscano alla spedizione di Pollione in Dalmazia, è molto difficile per le ragioni cronologiche che noi abbiamo già illustrate: quella spedizione è dell'anno 714, mentre Elvio Cinna, se è vero il racconto riferito dagli antichi, fu ucciso dopo la morte di Cesare, nel 710. Perciò sarà da pensare ad altro viaggio di Pollione, fatto forse per ragioni di studio, nella Grecia. Da una lettera infatti di Cicerone a Lentulo, lettera che è del principio del 698, si trae che intorno a quell' epoca Pollione viaggiava nella (irecia; ed era giunto o era aspettato nella Cilicia, di cui era governatore Lentulo (2).

Del resto del riaggio cantato o descritto nel Propempticon di Cinna abbiamo due importanti testimonianze in due frammenti del commentario che Giulio Igino fece a quel componimento. I due frammenti ci sono conservati da Carisio; e ci apprendono l' uno che i naviganti dopo essere giunti dal promontorio Azio all' istmo di Leucade si facerano ivi trasportare da navi di rimorchio; l'altro pone il quesito, per qual ragione non si andasse diretta-

(1) Comm. in honorem Mommseni, p. 352.

(2) Fam. I, 6: «Quae gerantur accipies ex Pollione, qui omnibus negotiis non interfuit solum sed praefuit ». Poichè la lettera ¿̀ del principio del 698, il Kiessling, l. c., ne argomenta che Cinna dovè scrivere il Propempticon nella Bitinia, dove si era recato nel principio del 697, al seguito del pretore Memmio.

C. Pascal 
mente da Corcira a Leueade, piuttostochè fare diversione per il promontorio Azio (1).

Dai versi sopra riportati, ore à aceenno alle cose degne di essere viste e dalle parole del commentario di Igino, ore sono consigli eirea l’itinerario da seguire, si può dedurre che il Propempticon Pollionis manteneva lo schema consueto di siffatti eomponimenti: un augurio a chi si aceingeva al riaggio, menzione delle cose memorabili ehe egrli arrebbe risto. indieazione della via migliore, roti per il felice ritorno (2). Notevole ì che scrisse un

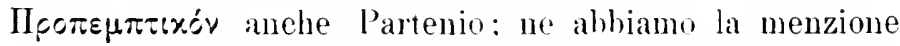
in Stefauo Bizanzio (3). Ora Partenio lo troviamo strettamente legato con la famiglia di Cinna: fu probabilmente il padre del poeta quel Cinna che feee prigioniero Partenio, nella gueria contro Mitrillate (1). $\dot{E}$ da pensare che il celebrato poeta green gorlesse presso la potente famiglia protezione e farore, e che non piceola efficacia egrli esereitasse sullattiviti poetical e sulle inclinazioni artistiche del giovane Cinna. Del resto questo genere di componimenti doveril essere aecetto ai poeti dello stil

(1) Carisio, 134 K.: Inlius Iygginus in Cinnac Propemptico:

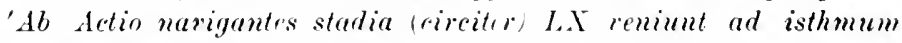
Loncadiensinm. Ibi solent iteris mimuendi causa romulco, quem Grace $\pi \dot{\alpha} \times \tau \omega v a$ dicunt, narem treshecere - "Quaerunt etiam nonnulli quam al rem a Corjenra inlerat Action narigare, quod est a regione tratuctionis Leucadirnsis, et rursus al Astio circa insulam moneat ire. quam "Corenra rectum itiner arl Leucatam ".

(2) Cfr. Vollmer a Stazio. Sill. III. "̈; Ilendrikson, Cluss. Journ.

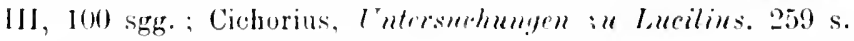

(3) Stefano Bizanzio, s. v. Kiogesxos.

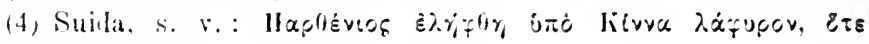

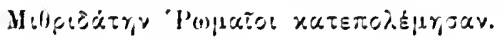


nuovo, giacchè anche gli alessandrini to averano usato (1).

Appunto per questo schema consueto dei Propemptica, si rende probabile che altri frammenti, che di Cinna ci son rapportati, si riferiscano al Propempticon Pollionis. Il frammento citato da Isidoro (2):

fr. VI. Atque anquina regat stabilem fortissima cursum

è un augurio di buona e sicura navigazione. Anquina, come spiega appunto Isidoro, è la fune, che tenera legata l'antenna all'albero maestro (3). Di augurii simili è naturalmente piena la letteratura di tal genere: cfr. ad es. Orazio, nell' ode sul viaggio di Vergilio in Grecia (4): "Sic te Diva potens Cypri, Sic fratres Helenae, lucida sidera, Ventorumque regat pater....., naris » e nel principio del Propempticon di Stazio a Mecio Celele tutta l'invocazione ed i roti per la navigazione propizia (5). Cotali augurii eran posti di solito in principio del carme, e cominciarano con una preghiera agli dèi, specialmente

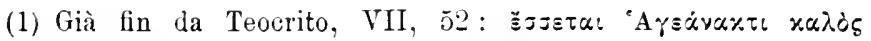

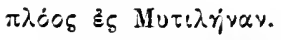

(2) Isidoro, Orig. XIX, 4, $7:$ : anquina funis quo ad malum antenna constringitur, de qua Cinna.... . I mscr. hanno regnat, regnant; leziono comune regit; Lindemann, L. Müller, Baehrens, Lindsay regat.

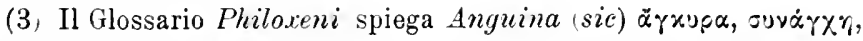
xúvaryos; ma è da ritenere la spiegazione di Isidoro. Anquina è l'attico árxolva; e la parola fu, per congettura, da antichi critici introdotta in un passo di Lucilio, ore contro la prosodia Nonio tramanda anchora soluta; vedi presso Weichert, op. cit. p. 192, Lucilii Reliquiae, v. 1114 Marx, e vol. II, p. 353.

(4) Orazio, Od. I, 3, $1 \mathrm{sgg}$.

(5) Stazio, Silvae III, 2, 1 sgg. 
a quelli del mare: il rerso nostro rappresentava quindi probabilmente uno dei roti formulati in tale preghiera.

$$
\text { ** }
$$

Alla indicazione di specialità naturali di qualche località, che si inviti a visitare si riferisce forse l'altro frammento (1):

fr. VII. Atque initata nives ludens legitur crystallus.

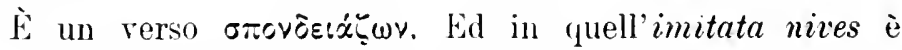
un'allusione etimologica: il poeta pensa a xpúos 'gelo',

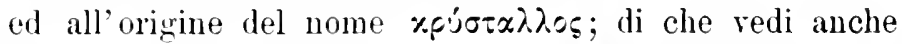
Plinio (H. N. XXXVII, 2, 10): "Non ulibi crystallus reperitur, quam ubi maxime hilsernae nives rigent et glacies, unde et nomen Graeci dedere»(2).

$$
\text { ** }
$$

Altro verso anche si riferisce ad un particolare della navigazione, e quindi è anche, con qualche probabilità, da attribuire al Propempticon; il seguente (3):

fr. VIII. Lucida confulgent summi carchesia mali.

(1) $\grave{E}$ conservato dallo Scoliaste a Giovenale, VI, 155 (grandia tolluntur crystallina): "Sic rt Cima licit: 'atque imitata nives ludens legitur crystallus'». Non veggo necessità di mutare ludens in lucens (Rutgers, Baehrens) o in ridens (Müller, De re metr. 143 ; cho peró pone lucens in Cimnac fraym., $7=$ ediz. di Catullo, 1. 88), 0 in undis ( $\mathrm{X}$. Heinsius). Ludens è immagine poetica : indica $\mathrm{i}$ riflessi di luce, gli schersi che fa il cristallo al solo.

(2) Vedi a tal proposito i sette epigrammi di Claudiano sul cristallo (I,VI-IXII fiesnor; Carm. min. XXXIII-XXXIX Koch = 1. $249-251)$.

(3) Isidoro, Orig. XIX, 2, 10: c Carehesia sunt in cacumine arboris trochlore, quasi fr littra, per quas funes trahuntur. Cimna: 
Carchesia sono le rotelle alla sommità dell'albero, per mezzo delle quali sono tirate le funi: per l'attrito continuo esse erano lucenti. Il poeta descrivera quindi all'amico il momento in cui la nave si apparecchiava a salpare e veniva sciolta dal lido: per maggior vivezza di rappresentazione il poeta si raffigura presente dinanzi agli occhi la nave in partenza: il verso poteva forse appartenere ad una serie di proposizioni descrittive, introdotte con un ecce o simili. Questo verso non tutti ammettono sia di Cinna. Alcuni anzi, come il Mïller, lo espungono dai frammenti e lo attribuiscono a Catullo. La ragione è che appunto a Catullo viene attribuito un verso molto simile. Vediamo brevemente di questa questione.

Nonio (546 M.) ha: Carchesia.... alias summa pars mali, id est foramina, quae summo mali funes recipiunt... Catullus veronensis: Lucida qua splendet carchesia mali ». Questa citazione ha riscontro con quella di Isidoro e dello scoliaste di Lucano che però attribuiscono il frammento a Cinna. Da ciò alcuni hanno concluso che sbagli Nonio, e che il frammento sia di Cinna (1); altri che sbaglino Isidoro e lo Scoliaste, e che il fram-

Lucida confulgent alti carchesia mali ». V. anche Scholia Lucani V, 418, p. 377 Web., che anche attribuisce a Cinna. Circa il simile frammento attribuito a Catullo, v. testo. Lo scoliaste di Lucano ha, invece di confulgent, cum fulgent, ed invece di alti, summi, che noi riteniamo, per il confronto con Lucilio; v. appresso.

(1) Cfr. ad es. Schwabe, Catulli Veronensis liber, p. 104, a framm. XI. Non si dichiara lo Ellis, Catulli Veronensis liber, p. 220, a framm. V; l' esclude dal numero dei framm. Catulliani il Friedrich, Cat. Veronensis liber, p. 61. 
mento sia di Catullo (1): ed anzi al frammento fu trovato posto nel carme LXIV di Catullo, dopo il verso 235. Ora è la osservare anzitutto che le due citazioni non sono identiche: nell’una è confulyent (Cinna), nell altra è qua splendet (splendent), e manca il sumemi (Catullo). La veriti ì che la fonte di tutto questo motivo poetico i Iucilio:

Tertius hic mali superat carchesia summa (2).

Il verso fu imitato da Cimna, che scrisse:

Lucida conlulgent summi carchesia mali.

Ya ciò non ruol dire che non possa essere stato imitato anche da Catullo, e che non debba attribuirglisi il frammento:

... Jucida qua splendent carchesia mali.

Fu poi imitato anche dis Lucano, (V, 418):

Hic utinam summi curret carchesia mali.

In conclusione niuna ragione $v^{\prime} \dot{e}$ per supporre che 1sidoro o Tonio o lo scoliaste di Lucino abbian preso

(1) Ad es. L. Müller, ediz. di Catullo, 1. 89: Quae idem Isidorus affert sub Cinnae nomine XVIIII, 2 et 33 ex Catulli carmine 64 sunt petita \$. Infatti il Miiller (p. 4ii) pone il verso nel carme LXIV, dopo il verso 235 (così gìi in antico il Faemo o il Mureto).

(2) Preferirei serivere summi. La citazione is in Nonio, 546. Vedi Lacilii Reliquiae, v. 1309 Marx (I, p. 90; II. 1). 418). Credo molto probabile (col Dousil) che tertius sia errore gratico per tertio. Il fr. apparterrehbe quindi al libro Ill di lucilio, nel quale il Cichorius, I'ntersuchunyen : L Lueilius, Berliu, 1908, p. 259, raviso lo tracce di un componimento simile ad un Propemptiom, ancanto a 'puelle della descrizione del viagrio in Sicilia. Cinna per il suo lromemptieon aveva dumpue modo di usufruire fualcher verso luciliano. 
abbaglio: i frammenti appartengono l'uno a Cinna, l'altro a Catullo: essi risalgono ad una fonte comune, Iucilio (1).

$$
* *
$$

Un rerso di Cimna conservatoci da Gellio (IX, 12, 12) è :

fr. IX. Somniculosam ut Poenus aspidem Psyllus.

Gellio cita il rerso dai poematn di Cinna: ed è probabile che si debba intendere di breri poesie, forse di carattere scherzoso ed amatorio. E lo cita per il significato di somniculosus, che egli pone nella serie degli aggettivi, i quali possono arere doppio significato: così formidolosus vale tanto qui formidat quanto qui formidatur; invidiosus tanto qui invidet quanto cui invidetur ecc.

Somniculosus vale dunque in questo ver'so qui sommum affert; in pari significato, e detto anche di serpenti, Lucano adopera sommifer (2). Si tratta dunque di quei serpenti il cui morso produce un torpore che genera la morte: ne parla Plinio nella Historia Naturalis (3).

(1) A dir vero, neppure per un' altra citazione di Isidoro da Cinna, che anche si ritiene errata, io credo sia da escludere ogni dubbio. Il passo è Orig. XIX, 33, 3) : "Strophium est cinguulum aureum cum gemmis. De quo ait Cinna: Strophio lactantes cincta papillas ». Ora in Catullo LXIT, 65 si legge: "Nou tereti strophio lactentis vincta papillas ». Abbaglio di Isidoro? Oppure uso, in Catullo o in Cinna, del verso del poeta amico? Cosi Vergilio usufrui interi versi di Tario (Macrobio VI, 1, 39; 40 ; VI, 2, 20); efr. Robertus Unger, Varii de Iorte Eelogae Reliquice, Halle a/S. 1870 .

(2) Lucano, IX, 701: «Aspida somniferam tumida cervice levavit 2 .

(3) Plinio, Hist. 1. XXXIX, 4. 
(ili Psylli, come risulta da molteplici passi (1), erano popolo africano, conoseiuti cone incantatori di serpenti. Nel rerso seguente " nel precedente era lunque il verbo che significara l'incantamento. La forma della comparazione ci puo far pensare che il passo si riferisea alle arti fascinatrici di qualehe Venere terena, ehe lo aresse stregato: ma non è discreto arventurar congetture.

$$
* *
$$

All'uso del rocabolo nanus dobbiamo la eitazione, fatta da Gellio (2), di due endeeasillabi di Cinna:

fr. $\boldsymbol{X}$. At nunc me Genumana per salicta

Bigis reda rapit citata nanis (3).

Questi rersi erano in poematis Helvi Cinnae, non ignobilis nerpe indocti poetae. Si trattara forse di brevi scherzi poetici, in forma epistolare, come quelli che troviamo frequentemente in Catullo (4). Il poeta vi narrava un suo viaggio per i eampi Cenomani, e eioè per la Gallia Transpadana, donde prohabilmente egli era nativo.

(I) Suetonio, Aug. 17; Lucano IX, 893 sgg. Plinio, H. N., VII, 2,2 ; VIII, 25, 38; Celso V, 27, 3.

(2) Gellio XIX, 13, 5, I versi si dicono addotti da un grammalieus latinus, per soddisfare alla lomanda di Festo Postumio, il quale voleva sapere se il rocabolo nanus " in quo de mulis aut ceuleis humiliorilus inlgo dicitur, anne latinum sil, et apud quem seriptum reperialur" ".

(3) Genumana hanno i codd, vioè Cenomana (cosi rijose il (ironovio). Vane sono le antiche congetture geniana, geniata, genuina; cfr. Weichert, op. cit. p. 199.

(4) Ad es. carmi XII, XXIII, XXXII, XXXV, XXXVIIl. 
Anche reda è parola di origine gallica (1). La reda era bigis.... citata nanis, cioè tirata da una coppia di muli; giacchè dal passo di Gellio risulta che tale è in questo luogo il significato di nani. È importante il notare che in questi versi Cimna mostra lo studio e l'imitazione di Ennio, che nei suoi Annali, faceva dire ad Ilia nel racconto della sua visione:

Nam me visus homo pulcher per amoena salicta Et ripas raptare.... (2).

$$
\text { * } *
$$

Come Catullo raticinava della Zmyrna di Cinna che i lontani secoli l'arrebbero letta (3), così Cinna vaticinava del suo amico Valerio Catone che la sua Diana sarebbe rimasta nei secoli. Suetonio ci ha conservato questo verso di Cinua (4):

fr. XI. Saecula permaneat nostri Dictynna Catonis.

Il verso era tratto da un componimento, che con probabilità possiamo immaginare del medesimo genere del

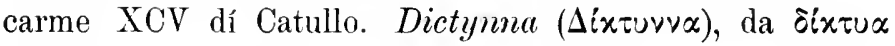
' reti', è il nome greco per significare la cacciatrice Artemide (Diana) (5).

(1) Cfr. Alois Vaniçek, Fremdwörter ir Grieehischen und Lateinischen, p. 78 .

(2) Presso Cic., De div. I, 20, 40. Ilia racconta alla sorella il sogno, nel quale le sembrò esser rapita da un homo puleher.

(3) Carme XCV, 6: "Zmyrnam cana diu saecula pervoluent».

(4) De ill. Gramm. 11.

(5) Eurip. Ipp. 146, Aristofane, Vesp. 368. 
Anche il titolo pare dunque indicare la derivazione greca, cioè alessandrina. Valerio latone, oltre i grammatici libelli, dei quali fa menzione suetonio (l. cit.) ed oltre le poesie amorose, cui accenna Ovidio (1), scrisse la Lydia e la Dictymna, probabilmente due epillii di genere alessandrino; e sulla Lydia Suetonio stesso riferisce un rerso di Ticida: Lydia doctormm maxima nura liber (2). Erano dunque anche per essa, come per la Zmyrna di Cinna, necessarie molte illustrazioni di dotti grammatici.

$$
* *
$$

Altri due frammenti ci sono stati conservati di Cinna: ma sono di molto scarsa importanza, ni si potrebhe dedurre altro da essi, che una preferenza del poeta per l'uso di parole ineonsuete. Ci sono entrambi conservati da Nonio. L'uno è

fr. XII. - vu - miseras andet galeare puellas

che Nonio cita per l'uso di galeare, da porre accanto a quello di rlipeare (3). Galeare ì armare di galea'. Non

(1) Ov. Trist. JJ, 436.

(2) La Lydia non i quolla sho tuttora abbiamo; cfr. TenffelSchwabe, Lïm. Litt. 200,2 . Il punta Tivida ì rammentato ancho da Ovidio (Trist. I1, 43:3), da Apuloio (Apol. 10); no i citato il nome acoanto a quelli di Furio Bibaculo e di Valerio Catono in Suotonio, fre gramm.; P'risciano (1). Lent. II, 186, 2) rammenta

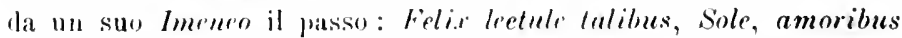
(a ragiono di sole vocativo di solus).

(3) Nonio, 87 (a proposito li rlipeat): a Ila et yalerere. Cinna in Epigrammalis.... 
è possibile naturalmente sapere di quali fanciulle guerriere si tratti. Il frammento, come attesta Nonio, apparteneva agli epigranmi.

L'altro frammento è:

fr. XIII. - vv, - vv, - vv, - Alpinaque cummis,

ed è citato da Nonio (1) per la forma femminile $1 \mathrm{mmm}$ is invece della consueta forma neutra cummi. In ureco i

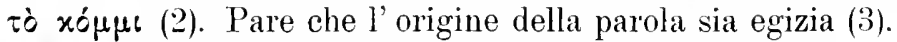
Cummis si ha anche in Catone (4). Ma in latino si ha anche gummi, gummis e gumen (5).

(1) Nonio, 202: "Cummi, generis neutri, ut cst usu, monoptoton. Cummis femminini. Cinna: 'Alpinaque cummis' (così gli editori : i codici cummi o gummi). I't sit genctivus eius huius cummis, ut puppis, febris, pelvis ».

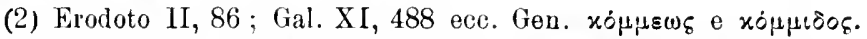

(3) Vaniçek, Fremdwörter, p. 27 ; Hehn, Kulturpflanzen² p. 184. Oppure araba? Cfr. Bailly, Dictionnaire gr.-franc., s. v.

(4) Acc. cummim, Catone, R. R. 69.

(5) Cfr. Georges, Lex. d. lat. Wortformen, s. v. (p. 183). 

III.

IL POETA ANSERE 

Il nome di Cimna ne conduce a quello di un altro poeta, sul quale anche abbiamo dagli antichi piì di una testimonianza, Anser. Oridio infatti in quella lista di poeti licenziosi, che egli apporta per iscusare la propria licenziosità, accomuna i due nomi, ma asserisce che più ancora di Cinna era procace Ansere (Trist. II, 435): "Cinna his comes est Cimnaque procacior Anser ». Se il paragone con Cinna qui è fatto sotto il rispetto della licenziosità, per contro in Vergilio è fatto nei riguardi dell' arte. Vergilio giuoca sul nome Anser, ed all' oca contrappone i cigni canori, simbolo dei poeti; e cigni sono per lui Varo e Cinna (Ecl. IX, 35):

Nam neque adhuc Varo videor nec dicere Cinua

Digna, sed argutos inter strepere anser olores.

Servio nel rilevare il giuoco di parola, che ì in quell'anser e l'allusione al poeta Anser, giustamente nota che un pari giuoco di parola era stato adoperato anche da Cicerone. Avendo infatti Antonio, protettore di Ansere, donato al suo poeta un campo nel territorio Falerno, 
Cicerone invocò che dal Falermo fossero scacciate le oche (1).

Che reramente nei versi vergiliani si abbia a redere nel nome anser una allusionc personale, si rende probabile per questo, che quei versi sono imitazione da Teocrito, ma Vergilio nel riprodurre la forma e il procedimento teocriteo, deliberatamente introdusse il paragone dell'anser, mutando il paragone teocriteo, che è tra le rane e le locuste. Si reggano i rersi di Teocrito (VII, 39 sgg.):

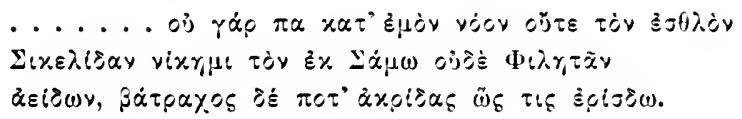

E il paragone vergiliano tu ripreso da Properzio in un luogo, ove si fa una enumerazione di poeti di amore, per auspicare che anche i proprii carmi non tornino ingrati ai lettori, o che essi sieno inesperti di amore o che ne sien periti (III, 34, 81 $=$ II, 25, 81):

Non tamen haec ulli venient ingrata legenti, Sive in amore rudis sive peritus erit.

Nec minor his animis, nec se minor ore canorus Anseris indocto carmine cessit olor.

Qual'è l'olor cui si allude in questi rersi? Non è dato saperlo: ma non mi par dubbio che eon anser si alluda proprin al poeta Anser, e che Properzio riproduca il giuoco di parole rergiliano, e, in questa parte della sua elegia

(1) Cfr. Servio, a Verg. Ecl. IX, 36: " alludit ad Anserem quendam Antonii portam, qui eius landes scribebat: de hoe stiam Cicero (Ihiliph. XIII, 11) " de Falerno Anseres depellantur. ipsum enim agrum ri donarat Autonius. 
or'è contenuto un inuo solenne alla gloria di Vergilio, voglia ripetere contro l'oscuro poetucolo il dileggio del grande Mantorano. Il significato dell aggettivo indoctum attribuito al carme di Ansere, risultil evidente da quel che sopra (p. 10 e segg.) abbiamo osserrato circa l'uso di doctus detto dei poeti: si tratterà di rersi ineleganti $\mathrm{e}$ rozzi. Del resto l'opposizione tra Ansere e Vergilio par che fosse non solo di gusti o di tendlenze poetiche, bensì anche di preferenze politiche e personali, se è da stare ad una informazione dello ps. Donato, secondo la quale la ragione dell'animositì dorrebbe cercarsi nel fatto che Ausere seguendo il partito di Antonio, doreva arrersare Vergilio (1). E fra i detrattori di Vergilio Servio menziona appunto Bario, Ansere e Merio, pessimi poeti (ㄹ).

$$
* *
$$

dnsere non è aunoverato da Catullo tra i saecli incommoda, pessimi poetae, contro i quali egli inveisce in più di un carme (3). Si è sospettato da alcuni che intercedessero tra i due poeti amicheroli rapporti; ed il nome di Anser si è voluto congetturalmente riporre in un passo molto guasto del libretto catulliano, in un passo anzi ove il restituire tal nome porrebbe subito Ansere in prima linea tra gli amici di Catullo. si tratta della fine del carme LXVIII." Ivi Catullo ringrazia con animo pieno

(1) Ps. Donato, V. Verg. 67: "Anser rero, quoniam Antonii partes secutus est, illum non observasse dieitur 》.

(2) Servio, ad eel. YII, 21: " ut sit Thyrsis Vergilii obtrectator, scilicet aut Bavius aut Anser aut Hevius, pessimi poetae 》.

(3) Carmi XIV, XXII, XXXII, XCV.

C. Pascal, 
di riconoscenza tutti quelli che gli han procurato il raggiungimento della felicita cui egli agognara, l'amore di Lesbia. Ha commosse parole per Allio, che ha offerto l'ospitale casa ai segreti convegui; indi soggiunge (115 sgg.):

Sitis felices et tu simul et tua vita;

Et domus, in qua nos lusimus et domina,

Et qui principio nobis + terram dedit aufert,

A quo sunt primo omnia nata bona.

Et longe ante omnes mihi quae me carior ipso est,

Lux mea, qua viva vivere dulce mihi est.

Qual'è la persona indicata nel rerso Et qui principio, ecc.? Le congetture sono numerosissime. Il Benoist, usufruendo in parte una congettura dello Scaligero e del Mitscherlich, in parte uma dello Heyse, legge: Et qui principio nobis te tradidit Anser. Anser arrebbe fatto conoscere Allio a Catullo: e di là a Catullo sarebbe derivato ogui bene. L'espressione potrebbe sembrare troppo enfatica ed iperbolica; ma ad ogni modo non occorre dimenticare che Catullo, per quei tali servigi di ospitaliti prestatigli da Allio ruol consacrare l'amico all' immortalità (1), il che sembra più iperbolico ancora. Il Kiessling, pur non proponendo congettura nel testo, crede che qui si alluda ad Alfeno Varo, a cui riserba l'ufficio di intermediario negli amori di Catullo e Lesbia! (2).

(1) C. LXVIII," 1-10.

(2) A. Kiessling, in Comment. in hon. Mommseni, p. 354. Omettiano l' indicazione delle altre congetture, per le quali rimandiamo ai commentarii critici; efr, ad es. il Commentaire del Benoist, p. 724-5. 
IV.

CELIO RUFO 

Due carmi catulliani (LVIII e C) sono indirizzati a Celio; altri due (LXIX e LXXVII) a Rufo; era naturale che si pensasse a MI. Celio Rufo, difeso da Cicerone con una famosa orazione nell' anno 698/56. M. Celio Rufo era stato l'amante proprio di Clodia, la Lesbia di Catullo, e da Clodia era accusato! Che Catullo, per designare il medesimo personaggio, abbia adoperato ora il nome ora il cognome, non può fare difficoltà: è il caso del poeta Licinio Calvo, che egli chiama Licinio nel carme L, Calro nel carme LIII. Ma ora son da esaminare i carmi catulliani diretti a Celio ed a Rufo: da essi infatti molti critici traggono l'impossibilità di riferirli ad un' unica persona (1). L'orazione di Cicerone pro Caelio ci darà

(1) Benoist, Comment. a C. LVIII (p. 510): "En tout cas le Caelius de la pièce $C$ ne peut être identifié arec Rufus des pieccs $L X I X$ et LXXVII, et il est plus vraisemblable que e'est lui dont Catulle fait le témoin de son mépris pour les debauches de Lesbie "; Heskamp, De Catulli vita ece. p. 28: * Hie Caelius Rufus (dell'oraz. Ciceroniana) confundendus non est cum illo Caelio, ad quem scriptum cst carmen LVIII: "C'aeli, Lesbia nostra, Lesbia 
molte notizie e tratti caratteristici, al lume dei quali meglio ci sarà dato di comprendere i carmi catulliani.

II. Celio Rufo fu giovane di molto ingegno e di buoni studii, ma corrottissimo di costumi. Volendo egli procurarsi nella vita pubblica autorità e potenza, scelse la più trista delle vie, quella dell'accusare: ma fu ripagato di pari moneta dal figlio di una sua rittima. L. Sempronio Atratino da lui accusato di hroglio fu assolto, ed egli ostinatamente tornò ald accusarlo. Allora il figlio dell Atratino trasse a sua volta in griudizio lui, presso il pretore Cn. Domizio Calrino. Erano capi di accusa l'avere eccitato in Napoli moti sediziosı, l'aver compiuto malversazioni nei beni di tal Palla, l'arere scacciato da Pozzuoli i legati Alessandrini, l'aver preso denaro a mutuo da Clodia sorella di P. Clodio. a averlo distribuito ai servi ti Lucio Lucceio, perche nccidessero Dione, capo della legazione alessandrina ed ospite ti Lucceio, intine aver tentato di far perire di releno Clodia. Lo stesso 1. Celio si discolpò delle accuse: si aggiunsero a sua difesa II. Crasso, che parlo delle prime tre accuse, e Cicerone che trattò delle ultime due. Dallorazione di Cicerone siano dunque informati piuttosto largamente dei rapporti che erano corsi tra Celio e Clodia. Cicerone mette in luce, senza veli e senza pieta, tutta la vita di corruttela, di libidine incestuosa, di sfrontata dissolutezza, che traeva Clodia nella sua casa del Palatino. Il giovane Celio, per la esuberanza dell età sua, per la indole in-

illa s, qui Veronae degebat, ut ex carminc entesima conchudi potest "; Otto Ribbeck, C. Valerius Catullus, eime litterar-historische Skizic, 1. 56, 11. 23: * Mass der Cölius ron r. 5.5 identisch sei mit dem liufus ron $e$. $7 T$, ist wirdcrum cinr piychologisirhe l'mmöglichlicit. 
cline ai piaceri, si era fatto irretire dalle arti di questa maga. Cicerone presenta tutto il processo come una trama infernale, ordita da Clodia, abbandonata dall'amante, per furia di gelosa vendetta. "Se già era sottentrata la discordia, egli dice $(25,61)$, se già era renuta meno ogni consuetudine, ed era spuntato il litigio, di qui certamente le lagrime, qui è da cercare la causa di tutti i delitti e di tutte le accuse ».

Più oltre $(31, \bar{i})$ egli cerca scusare, se non giustificare, il suo difeso per aver ceduto agli adescamenti meretricii di quella femmina. "Le voluttà per lungo tempo represse e costrette nell' età prima, talvolta d'improrviso si effondono e prorompono tutte». Ed egli assicura che ora il giovane suo difeso ha cambiato vita; ed anzi si è tanto staccato dall'infamia di quella relazione, da essere ora costretto a difendersi dall'inimicizia e dall'odio di quella donna $(31,75)$ : « tantumque abest ab illius familiaritatis infamia, ut eiusdem nunc ab sese inimicitias odiumque propulset $»$.

Le fasi di quest'amorazzo di Celio per Clodia, cioè per la Lesbia di Catullo, si rispecchiano nei quattro carmi dell'infelice poeta, dei quali due hanno il nome di Rufo, e due di Celio, e che noi reputiamo indirizzati tutti allo stesso personaggio, M. Celio Rufo. L'ordine di essi crediamo sia il seguente: LXIX, LXXVII, C, LVIII.

Il carme LXIX è uno scherzo. Non direi propriamente lo scherzo di un amico, come pensa il Riese (1) (sarebbe proprio il caso di ripetere: dagli amici mi guardi Iddio!); ma certamente non è neppure l'attacco sprezzante di un

(1) V. il suo Commentario a questo carme. 
rivale rittorioso, come pensa il Westphal (1). Ben altri complimenti riserbara il poeta ai suo nemici. Qui si tratta di spiegare a Rufo per qual ragione egli non abbia fortuna con le ragazze. per quanto cerchi vincerle con loni preziosi. si è diffusa la fama che errli mandi odor li capro dalle ascelle: e il capro ì una mala bestia, con cui una hella fanciulla non ruol giacere. Non r'è lo slegno di un nemico; $v^{`} \dot{e}$ il riso allegro di chi si sa preferito. Amico "nemico che fosse Rufo, non egli certamente aveva rivelato a Catullo tali particolari intimi.

Tali particolari sono rivelati lalle femmine, che dilegwiano sli spasimanti respinti, per rantarsi con gli amanti preferiti di averli respinti. Che tra le femmine cui nel carme si accenna fosse Clodia, nel carme non è detto. Va come non pensare ad essa, quando si sa che di essa arsero e Celio e Catullo? E vi fu un epoca in cui Lushia protestava non volere altro amante che Catullo, neppur se Giove la desiderasse! (2) Si, ma le promesse di una domma. " meglio di ma tal domma. bisogna scriverle sui ronti e sulle acpue dei fiumi: in ernto et rapida scribere. oportet aqu" (3). Clodia cappituli dimanzi a Celio. Ed ecco il grild straziante del arme LXXVII: " Rufo, che indarno e a torto io stimai amien (indarno: anzi con mio gran danno e scianuma). cosi tu hai strisedato sino a me, nd abbrueiandomi le viseere hai lapito a me misero tutti i miei heni: me li hai rapiti, ahimi. crudelo veleno della mia vita, ahime, peste della nustra amicizia! *. È m

(1) ratulls Gidichte in ihrem geschichtlichen Zusammenhange, 1. 136 .

(2) C. $1, X X, 1-2 ; 1, X], 1-2$.

(i) (:. IXX, 1 . 
acerbo rimprovero della tradita amicizia, un appello ai sentimenti antichi, una supplicazione dolorosa ed implorante. Ma il tono di tutto il carme mostra che il poeta crede ancor possibile la resipiscenza e la riconciliazione.

Passiamo ora al carme C. Si tratta di un orribile sodalizio fraterno, che il poeta chiama per ironia 'veramente dolce'. Celio anı pazzamente Aufileno e Quinzio Anfilena: Aufileno ed Aufilena sono fratello e sorella: ed il poeta.... fa gli augurii a Celio (che ama il maschio, Aufileno), perchè questi gli addimostrò coi fatti la sua unica amicizia, quando una fiamma insana gli abhruciava le midolla. Tutti questi personaggi del fraterno sodalizio sono di Verona, sono anzi il fiore della gioventì veronese (v. 2 flos reronensium... invenum).

si ritiene da alcuni (1) che non possa trattarsi del nostro Celio, perchè la scena qui si pone a Verona. Ma Catullo dice solo che i personaggi erano Veronesi, non altro (2). Donde fosse nativo Celio Kufo noi non sappiamo

(1) T. i commentarii di Baehrens e Schmidt; ed Heskamp, De Catulli Vita ecc. p. "si: * Hic Caelius Rufus confundendus nou est cum illo coclio, ad quem scriptum est carmen LXIII (sic, correggi l. $\mathrm{V}^{*}$ III), qui Ieronae degebat, ut ex carmine centesimo coneludi potest \#.

(2. Il flos Veromensum iuveurm si riferisce grammaticalmente ai nominativi Caelius e Quintius; ma probabilmente anche Aufileno ed Aufilena erano Veronesi, giacchè a Verona e nei dintorni sono frequenti nelle epigrafi i nomi Aufillenus, Aufillenu (sic), come fu rilevato dallo Schmidt. Prol. p. XLVI. V. il mio articolo in Athenceum, Ott. 1915, p. 445. Che l'oratore Celio Rufo fosse di Verona non sappiamo da alcuna fonte: dobbiamo ammetterlo, se lo identifichiamo col Celio di Catullo. Che l'uratore Celio fosse di Puteoli fu crronea supposizione derivata da una falsa lezione presso Cicerone, Cael. 5; r. Grocbe, in Hermes, 1901, p. 614. 
da alcuna fonte: neppur dunque la qualifica di "Veronese". che Catullo dà a Celio, costituisce un ostacolo per identificarlo con Celio Rufo. Ed a Celio Rufo ben s'attaglia quello che nell'epigramma Catulliauo è detto dell'amore per un maschio: giacchè Celio stesso scrive a Cicerone che si tentara trarlo in giudizio per tali accuse, secondo la legge Scantinia de renere nefanda ed il principale istigatore era Appio Claudio censore, il fratello appunto di Clodia (1)! Ed ora... sorroliamo sull' augurio di Catullo, che gli tocchi buona ventura in cotali amori (2); fermiamoci invece sulla ragione di gratitudine che egli ne adduce. "La tua unica amicizia mi si addimostrò coi fatti, egli dice (vr. 5- $\mathbf{7}$ ). quando una fiamma insana $a b-$ bruciava le mie midolla».

Niun dubbio che la fiamma insana sia l'amore per Lesbia. Quale sarì dunque questa prova di un' amicizia unica più che rara, che Celio dette a Catullo, mentre più ferveva impetuoso l'amore di costui per Lesbia? Il Thomas scrive: s'agit-il d'une aide, comme celle d'Allius, LXVIII," 26 et suiv, on simplement le conseils et de

(1) Cic. Ham. VIII, 12 (Caelius Ciceroni): "Postca non destitit (Appius Claudius) arcessere Polam Serrium, accusatorem, inire cum Domitio consilia. Quibus cum parum procederet, ut ulla lege mithi ponerent accusatorem. compellari ea lege me roluerunt, qua dicere non poterant. Insolentissimi homines, summis Circensibus ludis meis, postulundum me lege Scantinia curant. Vix hor erat Polce elocutus, quum eg" Appium Censorem eadem lege postulari. ('uorl molius caderet, nihil ridi.... Bel modo di difendersi, quello di ritorcere l' accusat: Ma che la vita di Celio fosse inquinata di libidini, accenna anche Vaterio Massimo, M. 4, 2, 7 . Cfr. Cic. I'ro C'uel. 3.

(2) Carme C, 5: Cui faveam potius? Caeli, tibi... 8: Sis felix, caeli, sis in amore potens. 
consolations? nous ne le savous pas » (p. 782) (1). Si, ma noi possiamo però sapere che certamente non si trattava nè dell'una cosa nè dell'altra. I consigli e le consolazioni somo parole, non facta; e quanto poi all'ipotesi che Celio abbia facilitato e reso possibili i rende:-rous, come Allio, tra Catullo e Lesbia, ria, si pensi che si trattava dell'antico amante, odiatissimo ormai dalla donna, e da lei accusato di reneficio. E ad ogni modo, quando pur si voglia credere anteriore la passione di Catulio per Lesbia all' amorazzo di Celio, l'offrire ospitalità per un conveguo di amore, è veramente una prova unica di amicizia? Naturalmente in siffatte cose è impossibile addurre tale ipotesi, che si converta in certezza; occorre contentarsi di un grado maggiore di probabilità. A me pare che l'accenno catulliano ad una prova di amicizia unica debba riferirsi ad un fatto molto importante per il poeta. Noi abbiamo visto come egli si rivolgesse all'amico in tono di rampogna ed insieme di supplicazione dolorosa, rimproverandogli il tradimento, che or gli avvelena la vita; abbiamo auche visto come ad un certo punto avvenne una completa rottura tra Celio e Clodia, e come quegli ebbe a sperimentare in seguito le furie gelose e le trame accusatrici della terribile donna; che appunto la rottura con Clodia non sia stata gabellata da Celio all' amico come un atto di amicizia per lui? Questa è

(1) I. Fenner, Quaestiones Catullianae (Barmen, 1896), p. 15, interpreta cosi questi versi : * Caelius quidam maxime videtur hortatus esse poetam ut amorem indignum abrumperet, de quare conferre liceat $c$. $C$, ' $v$. j sqq. " . Con tutto quel po' che precede nel carme? Da qual pulpito sarebbe venuta la predica! E che profitto ne avrebbe cavato Catullo, rompendo bensi l' amore indegno, ma augurando all' esortatore buona fortuna negli amori maschili! 
una ipotesi, e forse anche meno, una interrogazione soltanto: ma non è dato saperne di più.

Aggiungerò che ad un'amicizia per tal guisa e per tali ragioni riconciliata fa anche pensare il tono dell'ultimo epigramma diretto a Celio, un terribile epigramma, dal quale esala tutta l'amarezza del poeta, nel vedere Lesbia prostituita all'abbiezione estrema (c. IVIII):

Caeli, Lesbia nostra, Lesbia illa,

Illa Lesbia, quam Catullus unam

Plus quam se atque suos amavit omnos,

Nunc in quadruviis et angiportis

Glubit magnanimos Remi nepotes.

Lesbia nostra! Si ha un bello attennare il valore di quel nostra, e ritenerlo pari ad un mea. Ma precede il vocativo Caeli, e il nostra non dova riferirsi ad entrambi? Altri suppone il nostra detto ironicamente: Catullo rinfaccerebbe a Celio il suo anorazzo per Lesbia. Ma qui tutta l'amarezza non è contro Lelio, è contro Lesbia; e se essa or si c̀ data al rolgo dei trivii e degli angiporti, che importa or piì al poeta linferlelti con Celio? A me pare che il carme suppongal entrambi liberi ormai dai lacei di Lesbia, e ritornati amici, e Catullo desideroso, per la espansività di sua indole, di aprirsi con l'amieo, che anch'egli sat le tempeste: indi il nostra. Catullo or pui rammentare senza ire gelose che Leshia is stata di entrambi. So in principio della passione egli si imponeva di non essore geloso per nom diventare molesto (1), ora egli drer non esser geloso, per l'abbiezione ultima di Lesbia (r) pui comsolarsi con l'anico, con l’antico rivale.

Questa comdizione psicologiea sembra strama? Ma Pro.

(1) Carme 1, 1311 , 1 95. 
perzio, quando vuole persuadere il rivale Gallo a non insidiargli l'amore di Cinzia, gli rappresenta i pericoli futuri di quell' amore, e gli annunzia che invano Gallo spererà di trovar conforto in lui: deporranno le lagrime l' uno nel seno dell'altro, ardenti di un solo amore! $(\mathrm{I}, 5,27-30)$ :

Non ego tum potero solatia ferre roganti, Quum mihi nulla mei sit medicina mali : Sed pariter miseri socio cogemur amore Alter in alterius mutua flere sinu.

Quello di Catullo e di Celio per Lesbia non era stato un socius amor, giacchè anzi il poeta ne areva aruto fiamme iraconde di gelosia: ma ora entranbi erano fuori di quell' amore, che però dava ancora a Catullo scatti e fremiti sdegnosi di rampogna $\theta$ di rimpianto. 

V.

MAMURRA E CESARE 

In quattro dei suoi epigrammi satirici (1) si scagila Catullo contro un ignoto personaggio, che egli chiama Mentula. Il cognome è molto significativo. Ma si tratterà di un rero cognome o di un insulto lanciato dal poeta arguto e licenzioso? $\mathrm{E}$ in ogni caso di un cognome o di un nomignolo? A me pare che il primo dei quattro epigrammi dia una soddisfacente risposta. Esso è:

Mentula moechatur. moechatur mentula: certe.

Hoc est, quod dicunt, ipsa olera olla legit (2).

Evidentemente olera olla legit, è uno di quei proverbii popolari suggeriti dall'allitterazione e dalla pretesa di etimologizzare. Varrone infatti (L. L. V, 108) pone appunto la falsa etimologia: ab olla olera dicta. Il proverbio letteralmente significa: 'la pentola accoglie gli erbaggi".

(1) Carmi XCIV, CV, CXIV, CXV.

(2) Non c'è varietà di lezione degna di nota. La punteggiatura da noi adottata è quella dello Scaligero. Lo Schwabe congiunge: moechatur mentula certe. Il Koch (Symbol. philol. Bonn. p. 320), il Baehrens e il Riese: mocchatur mentula. Certe Hoc est....

C. Pascal

8. 
Il che corrisponde presso a poco a quel che noi diremmo: ' la botte dà del vino che hal'. Ma se si pensi alla etimologia olla olera, ed al significato del proverbio, bisognerà aggiungere un altro pensiero accessorio, che noi potremmo esprimere con un altro proverbio latino, c cioc: respondent rebus nomina saepe suis. Con queste due spiegazioni apparirì chiaro il significato delle parole: 'l' olla raccoglie olera'. Trasportando te due idee al caso di mentula e Mentulu: "Mentula non puó fare se non ciò che può fare ma mentula cioc 'commettere adulterii'. Deve clunque trattarsi di uno, che avea per nome o nominnolo Mentula e il poeta dice che gli è bene appropriato. Il cognome Mentula non ènella letteratura nè nelle iscrizioni: è dunque più probabile che si tratti di $u n$ nomignolo, probabilmente appioppato dal popolo, o, se si tratta di Mamurra, dai soldati, durante le spedizioni di Cesare, nelle quali Mamura fu capo della ingegneria militare.

E che di Mamura veramente si tratti credo anch'io, perchè nel carme XXIX Catullo rivolinemlosi a Cesare e Pompeio chiama nuovamente montula Mumura (v. 13): nt ista vostra diffututu mentulu. I soldati, come non furono avari a Cesare di sangunosi epigrammi, così certo neppure alle persone lel sun somuito lesinarono lazzi e mottergai e nomignoli offensivi. Del resto i particolari che di Mentrela ci dice Catullo corrispondono a un dipresso a quelli che egli ci rappr a di Mammra.

Questi i un impudien vorace e biscazziere, che va girando da letto a letto (XXIX. 2 e $\bar{c}$ ), ¿ un adultero, compagno e insieme rivale di Cinare in amori di sgualdrinelle (LVII, S-!); o come un moerhus i presentato Mentuln nell'epigramma XCll', al altrove (CXV, s) come

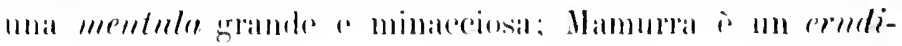


tulus (LVII, 7), con che si ruol forse indicare che egli si piccara degli studii di poesia (1): Mentula si sforza di salire sul monte delle Muse, ma queste lo ricacciano in giù con le forcine (c. CV); Mamurra è un dissipitore di patrimonii: egli ha dirorato prima il patrimonio paterno, poi la preda pontica, poi l'iberica, ed ora attenta alla gallica ed alla britamica (XXIX, 16-22); egli è anzi, per antonomasia, il decoctor di Formio (XLI, 4; XLIII, 5); ed anche Mentula si trova a mal partito: anzi nei suoi immensi possedimenti, nel Firmamus saltus, i pesi superano i redditi $(\mathrm{CXIV}, 4)$. È stato obbiettato che dappertutto Catullo prende di fronte i suoi nemici e rivali e li indica col loro nome, salvochè per Lesbius (c. LXXIX), psendonimo naturalmente suggerito dall'altro, Lesbia, adottato per la sorella; ed anzi il Jungclausen (2) ritiene che la riconciliazione già arvenuta con Cesare (3) aresse indotto il poeta a continuare più velatamente i suoi strali contro Mamurra; mentre d'altria parte il Fröhlich nega assolutamente l'identificazione con Mamurra, si perchè, come abbiamo detto, Catullo non areva ritegno di colpire di fronte i suoi nemici, si perchi in ogni caso nou si ravrisa, a suo giudizio, quali tratti così spiritosi possano

(1) $O$ anche degli studi grammaticali, ciò̀ di critica dei poeti. Anche Cesare, cui pure si riferisce il dileggio catulliano (erudituli $a m b o$ ) fece poesie ed un trattato grammaticale. La forma diminutiva consiglia nel verso LVII, 7 ad interpungere: Uno in lectulo, erudituli ambo. Non ponendo la virgola, si verrebbe a dire che Mamurra e Cesare sono saccentuzzi nel praticare in un sol letto la mutua libidine: ma a ciò l'idea diminutiva del saccentuszo non si addice.

(2) Chronol. der Cied. Cat. 1, 22.

(3) Suetunio, Caes. 73. 
avere questi epigrammi, da rendere più ridicolo l'odiato nemico: sì perchè i caratteri che si dicono comuni ai due personaggi sono in realtì di un gran numero di personaggi in quell' epoca. Ognuno rede quanto sieno deboli e caduche cotali argomentazioni. Catullo colpiva di fronte i suoi nemici, senza bisogno di ricorrere a pseudonimi. E chi dice che rolesse nascondersi? Quando egli parlava del fallito di Formio, del deroctor Formianus (XLI, 4: XLIII, 5), senza nominarlo, tutti intendevano Mamurra: è venuto mai in mente ad alcuno che egli temesse di nominarlo? E così, se egli chiamò Mentula Mamurra o altro qualsiasi personaggio, dovè certo adoperare nomignolo ben conosciuto, appioppato a quel personaggio dalla impertinente e petulante salaciti di nemici, 0 più probabilmente di amici. L' adoperare quel falso nome, si dice, non i tal renere di spirito, che si convenga alla violenza degli attacehi contro Mamurra. Ma si tratta di uno, che Catullo rappresenta come dissipatore di sue sostanze in amori meretricii: egli è l'amico dell'Ametina puella defututa (XL), egrli è l'anico della puella nec minimo naso nee bello pede nec nigris ocellis ecc. (XIIII); egli è uno in lectulo con Cesare (LVII, 7), e con lui i insieme rivale e compagno negli amorazzi delle sgualdrinelle (LVII, 9) ; si dovrà credere poco conveniente a proposito di tal personaggio trarre qualche ragione di scherzo dal nomignolo Mentula, e addirittura appioppargli tal nomignolo?

Ma i caratteri, si osserva, sono comuni a tanti altri personargri di quell'etì. Sì, ma nol caso nostro si ha, come si è gria osservato, ma rispondenza specifica d'innegahil valore: in mun degli epigrammi contro Mamurra (e. XXIX 1:3), il poeta rlvolgendosi al suocero ed al genero, 
a Cesare ed a Pompeo chiama il loro protetto ista vostra diffututa mentula: si dovà credere anche qui ad un riscontro casuale o non piuttosto ad una parola intenzionalmente adoperata?

Due degli epigrammi contro Mentula, il CXIV e CXV, sono in dileggio delle sue vantate ricchezze. I suoi possedimenti lanno uccellagioni, ogni genere di pesci, e prati e campi e fiere; ma invano: i redditi non pagano le spese. Saran belli i fondi, ma il padrone è in malora. Ciò nel carme CXIV: e del medesimo genere è il carme seguente, ove però la punta finale è diversa: dopo arere magnificata l'immensità dei possedimenti, e certo per dileggiare ironicamente le vanterie e le iattanze del padrone, si conchiude che se tutto ciò è grandioso, piì grandioso ancora è il padrone non come uomo, ma come mentula. Ora questa derisione di rantate ricchezze e questo vituperio della povertà, è un tratto che Catullo adopera contro i suoi rivali fortunati. Egli insiste nel rammentare a Giorenzio che il suo amante non ha nè serro, nè scrigno (XXIV, 5: XXIII, 1); egli scherza sulla villetta di Furio, esposta al più terribile e pestilenziale dei venti, quello delle ipoteche (c. XXVI); egli si arrabbia perchè il fanciullo amato, e le cui grazie gli sono state ora insidiate da Aurelio, soffrirà sotto di lui la fame e la sete (XXI, 10-11).

Anche Mamurra era un suo rivale. Giacchè Catullo avera chiesto le grazie di una delle amiche di Mamurra, della puella Ametina: ma questa pretendeva, per concedersi a Catullo, diecimila sesterzii (XLI): apriti, cielo! Catullo consiglia subito di convocare un consiglio di medici e di anici, perchè la ragazza è malata: soffre di frenesia. Non che Catullo si macerasse troppo per il ri- 
fiuto di Ametina: anzi se Ametina è la puella nec minimo nuso ne bello pede ece. del carme XLIII (1) è dla credere che si consolasse col rilerarne i difetti fisici; ma hastara il rifiuto e la richiesta della grossa somma, perchè Catullo dileggiasse la ricchezza del rivale, cui Ametiua era prodiga di sue grazie, di quel decoctor, di quello spiantato di Formio. Naturalmente a questo piccolo motivo di carattere personale, a questo capriccio insoddisfatto, si aggiungevano, per rinfocolare le ire di Catullo contro Mamurra tutti i motivi di carattere politico e il generoso shegno, che prorompe nel carme XXIX contro gli sperperi e la potenza rovinosa di quell'uomo. Il Munro (2) tendera ad attenuare il significato degli epigrammi catulliani contro Jlamurra, quasi si trattasse di scherzi e di fescenmina iocatio, ma ninua attenuazione i possibile, per quanto riguarda il pensiero e le intenzioni di Catullo, giacchè egli scaglia accuse determinate e precise (3). Iolto pero si deve concedere all ardente passionalita del poeta, che ingraudisce colpe e pregi. vizii e virtì.

Certo non e prudente domandare ai poeti il giudizio sugli uomini e meno ancora sulle donne. Qui, nella rappresentazione Citulliana di Mamurra, non r'e una sola nota simpatica: tutto è dipinto a colori molto neri. Bisogna credergli: O anche qui egli si fece infiammare da tutte le mormorazioni e denimrazioni, che correvano per la dissipata ed oziosa societa nobilesea di Roma sugli nomini

(1) Cio si può supporre perchè l'una e l'altra è decoctoris amica Fopmiani, cioe amante di Mamurra. Ma è troplo debole indizio. Il turpiculo puella naso di XLI, 3 corrisponde al nec minimo puclla naso di XI,111, 1:

(2) Munro, 'ritir. and eluridat., p. 85 seng.

(3) Ern. von leutseh, in Mhilologus XL1 (1882), 1. 283. 
che più erano in luce per potenza e faror popolare, e specialmente sugli homines nori reuuti dalle provincie a far la lor fortuna in Roma? Mamurra fu praefectus fabrum di Cesare, e cioè capo della ingegneria militare (1). Si ammetterà facilmente che per le spedizioni di Cesare tal carica non potera conferirsi se non a persona di alto merito professionale: la rapidità delle operazioni, la difficoltà di opere come strade, ponti, acquedotti, fatti sopra ordini immediati, per scopi militari, ci garentiscono che egli dorera arere doti non comuni dingegno e di energia.

La stessa importanza della sua carica è indizio preciso che egli non doreva essere più giorane. Ciò rende a dir vero poco rerisimile l'accusa sanguinosa di Catullo, che egli si prestasse a far da bardassa (c. LVII).

Catullo, abbiamo visto, fu rivale di Mamurra, e rivale sfortunato. Queste disarventure gli coceran troppo, perchè egli risparmiasse la donna riluttante ed il suo amante farorito. Ed ecco, la domna prima desiderata e richiesta di amore, diventa brutta e malata di mente: essa è turpiculo puella naso (XLI, 3), forse anche è la fanciulla che non ha picciol naso, nè bel piede, nè nere pupille, nè dita affusolate, nè bocca asciutta, nè garbo nel dire (XLIII, 1-4), ed alla quale può contrapporre trionfalmente la sua Lesbia: "Ed i prorinciali dicono che tu sii bella? Ed osano paragonarti alla mia Lesbia! O età sciocca e stupida! » (ivi, 6-8). I provinciali! E tale era l'amante preferito da Ametina, il dissipatore di Formio, decoctor Formianus. Il quale, a furia di spendere e di spandere, dorè procu-

(1) V. il commentario del Benoist a c. XXIX (p. 439 e sgg.) ; v. anche Schwabe, Jahrbiecher f. Philol. CXVII, 1878, p. 261. 
rare probabilmente a Catullo più d'una di tali sconfitte: nò forse Catullo ebbe sempre modo di contrapporre, come questa volta, l'amore di Lesbia! Certo nel terribile epigramma XXIX contro i due potenti protettori di Mamurra, Pompeo e Cesare, par quasi che il ricordo più scottante e molesto per il poeta sia proprio quello delle avventure amorose del ricco e prodigo avversario, che ora superbo ed azzimato va facendo il conquistatore da alcova ad alcova (XXIX, 6-7), con i denari rubati nelle provincie. Non si ha dunque qui propriamente un epigramma politico: probabilmente il primo e più verace movente ̀̀ personale. Del resto la rampogna contro Pompeo e Cesare qui scatta violenta, ma più specialmente coutro Cesare, i) cinedo Romolo, l'imperator unicus, che si era spinto fin nell'estrema isola occidentale. sol perchè quella diffututa mentula divorasse due o trecentomila sesterzii. Anzi il poeta enumena gli sperperi e le depredazioni di Mamurra: prima il patrimonio familiare, poi la preda Pontica, è cioc certamente il bottino preso da Pompeo nel l'onto nella guerra contro Mitridate, dal 66 al 63 av. C.; poi la preda iberica, e ciò le ruberie che Mamurra potè fire nella spangna, dal 62 al 60 , durante il governo che di quella provincia ebbe Cesare: ed or si temeva pure per la preda galliea e per la britannica. Anche qui le esagerazioni sono evidenti. Mamurra avri fatto del suo meglio nel Ponto, dove era al soguito di Pompeo, e nella spagna, dove era al seguito di Cesare: ma avra aruto certamente non poehi e non poen zelanti ed avidi competitori nell' impresa, partecipi del hottino pontico e delle spoliazioni iberiche. Na la chiusa dellepigramma ò signilicativa (21-21): "l'ercho protegracte fuesto malanno? A che altro ir andi buono salvochi a divorare grassi pa- 
trimonii? Ed a tal titolo, o suocero e genero, che siete i più potenti di Roma, avete mandato tutto a soqquadro?" Quel che preme al poeta, quasi si direbbe, è di staccare una buona volta il favorito dai suoi potenti protettori: ed egli avverte che Mamurra niente altro può, nisi uncta devorare patrimonia, ove il può (potest) non è certo fraseologico, bensi anzi indica effettivo potere; e la mancanza appunto di ogni altro potere può avvertire i due potenti protettori che niun vantaggio debbono ormai essi aspettarsi da Mamurra.

\section{$*^{* * *}$}

In questo epigramma Cesare è chiamato con efficace insistenza (v. 5 e 9) cinedo Romolo. Il nome tel fondatore stesso della città, applicato a Cesare, è un fine tratto ironico. $\grave{E}$ possibile che il nome di Romolo si desse nei canti trionfali dei soldati romani ai più famosi capitani tornanti a Roma dopo le vittorie decisive per la patria. La notizia, che Livio dà, che questo appunto avvenne per Furio Camillo dopochè ebbe sconfitto i Galli (1), pui farci sospettare in quel 'Romolo' un' appellazione tradizionale dei trionfatori, celebrati quali salvatori di Roma. Naturalmente il titolo poteva prestarsi ad esser volto in dileggio dai nemici; ed abbiamo infatti notizie che servi piì volte a schermaglie politiche, contro importanti personaggi, che si vantavano quali salvatori della città.

(1) Livio, V, 49, 7 : "dictator recuperata ex hostibus patria triumphans in urbem rediit, interque iocos militares, quos inconditos iaciunt, Romulus ae parens patriae conditorque alter urbis haud vanis laudibus appellabatur . 
Lepido chiamava Sulla scuevus iste Romulus (1), il che pui significare che anche a Sulla, e forse appunto nei canti trionfali, fu appioppato il titolo, rolto poi a dileggio dallarversario politico: di Pompeo si disse che egli, priche a Romolo si atteggiara, ne arrebbe aruto la fine (2); c lo scrittore della declamazione contro Cicerone chiamava ironicamente Cicerone Romule Arpinas (3).

IIa l'ira di Catullo contro Cesare si sfoga con ben altre invettive. Egli ì - improbo cinedo 'pathicus, morbosus, vorace adultero, rivale con Mamurra negli amori di sgualdrinelle: su lui e su llamurra sta impresso un marchio d’infamia, che nulla giungerà a lavare (t) : egli è impurlico e vorace e bisciaziere (i). Non sarebbe prudente prendere alla lettera tutte queste accuse catulliane, o supporre in Catullo stesso l'onesta prudenza di non lanciarle, senza averle prima controllate. Suetonio (6) ci attesta che il soggiorno di Cesare alla corte di Nicomede fu l'unico fatto ehe intiecó lis sua fama. Ma anche di quel fitto che giustificherebhe l'accusa di cinedo, il testimone era il famigerato Memmio, e ripugna anche a noi presumere di scoprire la reriti da siffatte fonti (7). Sella procace liberti delle pompe trionfali poterano i soldati ripetere in senso scherzerole $l^{\prime}$ useeno accenno;

(1) Sallustio, Hist. 1, 4, 45 Oratio Lepidi, 5).

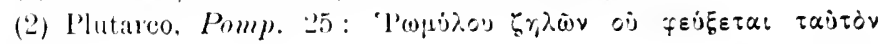
$\varepsilon \% \varepsilon i \nu(p) \tau \dot{\varepsilon} \lambda \circ \varepsilon$.

(3) Itrelam. in rir. IV, 7 (= sallustii in ciecromem et inrierm incertiene, rea. A. Kurfess. Liphine. Teubner, 1914, 1. 7).

(4) Tutte queste invettive sono nel carme ISII.

(5) C. XXIX, 1 " 11).

(b) Cass., 19.

(7) V. Ilange, tril. and slucid. P. si o segge. 
ma i Romaui in tal genere di tolleranza erano abituati a cotali scherzi, e facerano ostentazione di spirito superiore col sorriderne (1): quale sposo o quale sposa arrebbe potuto esser tranquillo, se aresse voluto prestare l'orecchio ai fescennini cantati nella cerimonia nuziale? $\mathrm{E}$ per quanto riguarda le altre accuse, niun ricordo o indizio ci è lasciato dall' antichità, che ralga ad arvalorarle; anzi gli antichi ne esaltano proprio quelle doti e quei pregi, che sono i contrapposti precisi dei vizii imputatigli. Certo la bellezza di sue sembianze e la diffusa prodigalità, con la quale egli spandeva il denaro che areva, ed anche quello che non avera, poterono dare appiglio a più di una roce maligna: ma niuna voce sorse ad intaccare le sue doti di sobrietà, di calma, di prudenza, di resistenza alle fatiche, di indomito rigore di animo, di moderazione nel sonno e nella mensa (2).

Queste accuse nascerano in quella società pettegola e maligna, uella quale schizzavano contro Cesare il veleuo della loro animosità politica e dei loro odii personali,

(1) V. Bernstein, Versus ludicri in Romanorum. Caesares priores p. XI-XIII, XIX-XX. (Halis Saxonum IIDCCCX). $\nabla$. anche la Praefatio apposta dall'Eichstaedt a quest'opera, p. X-XIII; ed Ettore Stampini, Alezne osservazioni sui carmi trionfali romani, in Riv. di Filol. XXVI, fasc. $2^{\circ}$, 1898. Probabilmente i carmi trionfali erano alterni. a botte e risposte, tra un coro di lodatori ed un coro di dileggiatori ; cfr. Bernstein, op. cit. p. XIII, e Stampini, op. e. p. 20 e segg. dell'estratto.

(2) Vell. Patercolo, II, 41; "forma omnium civium excellentissimus, vigore animi acerrimus, munificentiae effusissimus, animo super humanam et naturam et fudem evectus, maynitudine cogitationum, celeritate bellandi, patientia periculorum Layno illi Alexandro, sed sobrio neque iracundo, simillimus, qui denique semper et somno et cibo in ritam non in roluptatem uterctur. 
Ilemmio. Dolahella, Curione. Calro e gri altri nemici di Cesare. Il poeta prendera a rolo i lazzi lanciati leggermente. con pettegola petulanza. e ne facera tratti spiritosi ed acri di vilipendio. Che egli stesso prendesse molto sul serio questi suoi attacchi non è da credere. Quella stessa mobilità di sentimenti. quella stessa eccitahilità di passioni. che scorgiamo in lui nei rapporti di amore e nelle rampogne contro i rivali, quegl'impeti e quelle violenze, che si alternano a quando a quando con roci di rimpianto e di lamento e di affetto, ritroviamo nel suo contegno in riguardo ai nemici personali e politici. Se Roma ì tutta piena di ammirazione per Cesare, se tutti si affannano per mettersi in buona mostra presso di lui e riuscirgli graditi, solo questo giorane scapestratello e spensierato, ruol piantarglisi di fronte con aria sparalda e beffarda, e dirgli chiaro e tondo che a lui nulla gl' importa di C'esare e che non ruol neppure sapere, se egli sia bianco o ner" (c. XCIII). Quintiliano, rigido zelatore del nome di Cesare. cui si ricollegava l'imperiale dignità. se ne scandalizza. e chiama insania il motto faceto, e non ruole neppure citare per nome l'audace poeta (1).

Se Cesare si arlirava per le intemperanze Catulliane, piu baldanzoso il poeta muovera all attaceo: areva bollato Mamurra? ora si volge ad 1 in altro gruppo di anici e fautori del gran capitano: (Ottone e Libone e Futicio. e li accusa di sozza mutua lihiline. e rolgendosi poi in tono di stida a Cesale, Io provocal, ironicamente: 'arrab-

(1) Luintiliano, XI, 1, 3S: " Negut se magni facere aliquis poetarum "trum Carsar ater an allus homo sit. insania: rerte, ut idem Cacsar de illo direrit, adrouguntia est ". 
biati dunque una seconda rolta coi giambi miei incolpevoli, o unico capitano di eserciti!» (LIV, 6-7). Imperator anice! (1) Unice imperator! (2)

Il poeta ripete, quasi per burlarsene, la lode universale: con la stessa spensieratezza con la quale scaglia le accuse, nega l'evidenza dei fatti.

Non però soltanto l'ira di Cesare dovettero provocare le acri invettive Catulliane: è da credere che Cesare ne provasse anche acuto dolore. se è vero che egli non nascose di essere rimasto in eterno bollato dai versi Catulliani sopra Mamurra. Ce lo attesta Suetonio, e riferisce le parole di Cesare così: "sibi... perpetua stigmata inposita» (3). Arrà Cesare ripetuto in altra forma il pensiero stesso del poeta, quando a Cesare ed a IIamurra gridava (4):

Nec mirum: maculae paris utrisque,

Urbana altera et illa formiana,

Inpressae resident nec eluentur.

Anche l'epigramma CXIII investe Cesare, e piì ancora di Cesare, Pompeo, di cui deride le familiari disyrazie (5).

(1) XXIX, 11.

(2) LIV ,6-7:

Irascere iterum meis iambis

Inmerentibus, unice imperator.

(3) Suetonio, Cesare, 73.

(4) LVII, 3-5.

(5) Carme CXIII.

Consale Pompeio primum duo, Cinna, solebant

Macillaw : facto consule nunc iterum

Manserunt duo, sed ereverunt millia in unnm

Singula. Fecundum semen adulterio.

Mucillam è correzione del Pletner (i cod, Mecillam o Mecilia). V. Schwabe, Quaest. Catull. I, p. 213. 
Si tratta ivi di Mucia, moglie di Pompeo, e figlia del grande giureconsulto Q. Mucio Scevola. Pompeo poi la repudiò per la sua vita libertina nel $6: 2$ d. C. (1). Il diminutivo IIucilla i adoperato per dileggio. ed era consueto ad indicare donnette di facili amori (2). L'epigramma nel primo distico allnde ai due amanti di Mucia ; nel secondo distico annunzia che dal primo al secondo consolato di Pompeo gli amanti sono cresciuti a duemila. Il primo consolato di Pompeo è dell anno 70, il secondo dell'anno $55 \mathrm{av}$. C.; il farto consule nunc iterum del v. 2 ci attesta che proprio dell'anno 55 è l'epigramma. Or quali sono i due amanti dell anno 70? Uno è probabilmente proprio Cesare, che suetonio cita come amante di Mucia (:3). Chi ripensi alle parole del carme LVII, ove Cesare e Mamurra sono presentati come ricales socii puellularmm (v. 9) può suppurre che l'altro sia Mamurra. Ad ogni modo in questo epigramma Cesare non ì nominato: ma il poeta parla di quelle due prime infedeltà di Incia, come di fatto notorio, per il quale neppure occorresse apportare i nomi : e l'allusione è quindi evidente. IIa la scalmana di Catullo contro Cesare dura poco. I temperamenti passionali e li mrande eccitabiliti nerrosa passano facilmente dall' udio all amore o da questo a quello. Se nel 55 ar. C. Catullo facera sanguinose allusioni agli ardulterii di Cesare, gria nel $\bar{t}$ egli esaltara il "gran Cesare » ed i monumenti di vittoria da lui lasciati

(1) Cfr. Plotner, Q. Talerius Catullus, Epigr. in Iul. Caes. und Mamurra. Spire, 1849; Schwahe, 'm. Cat. I, 1. 217.

(2) Cfr. la lista di nomi fomminili diminntivi contenuti in una lettera di Antonio al ottavio presso Suetonio, Aug. (i9).

(3) Simpt., Caes., 50. 
nella Brettagna: Caesaris visens mommenta magni (1).

In quel tempo la riconciliazione era grià arrenuta. Suetonio ci dice che Catullo fece attestazioni soddisfacenti a Cesare, e che questi lo invitò a cena, e continuò a ralersi, come avera fatto per lo inmanzi, della ospitalitì del padre di lui (2). Dorettero certo essere amici comuni ar intercedere ed a procurare l'incontro tra i due uomini. Cesare del resto, con gesti accorti di signorile superiorità, sapeva attrarre a poco a poco tutti nell' orbita sua. Probabilmente l'incontro arremne nella primavera del 54, nella Gallia Cisalpina, che Cesare traverso di ritorno dall'Illiria (3). Nè a Cesare o a Mamurra si trovano altri accenni dopo quell' epoca: il poeta avrà di qui in poi, per i pochi anni che ancor gli rimangono, più intime angoscie e più segreti rovelli, che gli attristeranno la breve vita.

(1) Carme XI, 10. Cfr. v. 11-12: Gallicum Rhenum horribile acquor ultimosque Britannos ». Lo Schwabe riferì il carme al $699 / 55$, epoca della prima spedizione 1 i Brettagna; ma giustamente notò lo Ellis che le parole di Catullo sembran riferirsi ai grandi successi riportati da Cesare nella seconda spedizione, nel $700 / 54$.

(2) Suetonio, Cesare, 73: "Valerium Catullum, a quo sibi rersiculis de Mamurra perpetua stigmata inposita non dissimulaveret, satis facientem eadem die adhibuit cenae hospitioque patris eius, sicut consuerat, uti persereravit 》.

(3) Cfr. Bell. Fall. T, 2, 1. Il Nunro (Critic. and elucid., p. 80) congettura che gli assalti contro Mamurra cominciassero alla fine del 55 a. C., giacchè probabilmente in quell' epoca Mamurra, come praefectus fabrum di Cesare, era nella Cisalpina a raccogliervi i materiali per la costruzione di una nuova flotta. Dalla fine del 55 alla primavera del 54: l'impeto di furia aggressiva contro Cesare e i suoi durò solo un inverno: a primavera Cesare è già il gran Cesare (XI, 10). 

VI.

L'IRONIA CATULLANA E L'EPIGRAMIIA A GICERONE 

L'ironia di Catullo è così fine, che non sempre è dato coglierla e rarrisarla: e per alcuni passi anzi si può dire che essa tuttora ama nascondersi e dissimularsi : critici e filologi malamente, a nostro arriso, interpretano come dette sul serio alcune cose dette per celia o per sarcasmo: il poeta par burlarsi anche dei suoi lettori, dopo parecchi secoli. Di ciò apporteremo qualche esempio subito qui appresso. Per ora crediamo opportuno notare una delle caratteristiche dell' ironia catulliana. È questa: di ripetere le frasi preferite, o i modi o i mali rezzi, delle persone contro le quali si scaglia il sarcasmo o il dileggio del poeta. Il carme XXY è ad esempio contro il cinedo Tallo, che il poeta chiama più molle del pelo di coniglio: egli ruole rappresentarne al vivo la figura imbelle ed effeminata, l'andatura cascante, il fare e il dire sfiaccolato: ed eceo nei suoi versi tutto un seguito di diminutivi (XXY, 1-2):

Cinaede Thalle, mollior cuniculi capillo, Vel anseris merlullula vel imula oricilla 
Gli è che certamente Tallo stesso adoperara questo linguaggio tutto tenerume e mollezza (1). Orazio quando fa rappresentare da Tiresia la fincura del furbo, che tenta uccellare il testamento del recchio, usa per renderne le simulate tenerezze, forme diminutive simili (Sat. II, 5, 32): "gaudent praenomine molles anrimulae», (ivi, 37) «ire domum atque Pelliculam curare inbe». Si ha qui la rappresentazione ironica di vezzi o consuetudini, che rispondono al carattere delle persone. Ma un altro tratto di ironica rappresentazione è pur comune ad Orazio e Catullo; quello cioè di ripetere le parole stesse della persona dileggiata. Orazio, quando si ruol far giuoco di Furio Bibaculo, ne usufruisce il rerso Iuppiter hibernas cana nive ronspnit Alpes, sostituendo a Iuppiter, Furius (Sat. II. 5, 41) : ne importa addure altri esempii. E per quanto riguarda Catullo, che cosa significano, ad esempio, quelle appellazioni di Romule, di imperator unice rivolte a Cesare in un sanguinoso epigramma? (c. XXIX e LIV). Romulus solevano essere chiamati nei canti trionfali i generali vittoriosi, che si davan vanto di aver salvato Roma (2): evidentemente il poeta ripete, a scopo d'invettiva sanguinosa, gli elogi entusiastici degli ammiratori (3). Cosi che cosa significano le amplificazioni iper-

(1) Il diminutivo in senso ironico ì adoperato spesso da Catullo per rappresentare lo effeminatezze degli nomini bardasso; efr. XXIX, 8 albulus columbus: LVII, 7 uno in lectulo, erulituli ambo.

(2) Vedi il commentario dol Benoi-t, 1. 446; o Schwabe, Neue Jahrb. f. Philol. 1878, 4, 1. 261.

(3) Bene il Benoist nel suo Commentario (1. 446), a proposito di imperator unire: . Catulle ici reprend malignement arec ironie cette appellation sans doute publiquement attribuée à César par un de ses partisans. . 
boliche del carme CXV? Si tratta ivi di Mentula; e di Mentula si dice che egli possiede

Prata arva ingentis silvas saltusque paludesque Usque ad Hyperboreos et mare ad Oceanum.

Ed il poeta osserra che tutto questo è, sì, grande, ma che egli è grandissimo, non uomo, ma mentula grande e minacciosa. La celia finale è una risposta ai vantamenti di Mentula: era certamente Mentula stesso che si: gloriava e ripetera di possedere tutto quel po' po' di roba ed il poeta pittoricamente ne riproduce i vantamenti.

Un altro esempio: il carme XI. Si tratta di una intimazione a Furio ed Aurelio. Essi sono incaricati di dire a Lesbia che vira pure coi suoi trecento adulteri e li slombi, e non pensi più a Catullo. Il messaggio è breve, ma non breve è il carme che consta di 24 versi. Ma la maggior parte di essi è occupata da una lunga allocuzione a Furio ed Aurelio: questi sono compagni di Catullo o che questi voglia recarsi sino agli ultimi Indi, ove le spiagge sono battute dalle risonanti onde dell' Oceano orientale, o agl' Oceani e ai molli Arabi, o ai Sagi ed ai Parti armati di saette, o fin là dove il settemplice Nilo colora le acque o che voglia traversare le alte Alpi, vedendo i trofei di Cesare, il Gallico Reno e il minaccioso mare e gli estremi Brittanni; tutto essi sono pronti a tentare insieme a Catullo, tutto quel che vogliano i Celesti.... Che cosa significa tutto questo? Dopo sì ampie dichiarazioni, che li rappresentano come pronti a qualunque sagrifizio per l'amico, il poeta li incarica di cosa ben piccola: un finto messaggio a Lesbia, perchè vada al diavolo, per dirla in gergo volgare.

Ma si tratta veramente di due amici? Entrambi i per- 
sonagri a uno solo di essi appaiono in altri componimenti (XV, X YI, XXIII, XXIV, XXYI): ma in nessuno di questi appaiono come amici; in mo di essi anzi, il XXVI, Catullo inveisce contro l'uno e contro l'altro con grossolane invettive; si può essere sicuri che anche tutte le dichiarazioni del carme XI non dehbono essere prese sul serio. Il poeta le rammenta, ma non perchè egrli vi creda, 0) ergli stesso faccia questo gindizio dei due compari: le rammenta solo per dileggio: e l'esiguitì del messaggio, jer cui profitta dell'opera loro, mostra appunto il conto che egli fa delle loro proteste di amicizia.

Con tutte quelle ampie dichiaraziom dumque il poeta ripete le proteste che $\mathrm{i}$ due amici facovano, e le ripete per ironia. Anche qui commenta molto bene il Benoist (p. 393): "Ou peut croire qu ici en les chargeant de sa commission, qu'ils aient éte on non les intermédiaires de Lesbie, le poète leur intlige un nouvel outragre. L'óloge qu'il fait de leur amitié est ironique ». Se Furio fosse proprio Firro Bibaculo, come sospettano aleuni critici (nom si hann peri indizii per accoyliere o respingere l'ipotesi), si dovrebbe riconoscere in quella lunga ed ampollosa enumerazione geografica la caricatura dei versi stessi di Furio, il quale cra come è noto, gronfio poeta (turgides. Alpimus lo ehiama (Orazio); Catullo li arrebbe usufruiti o parafrasati ironicamente nel carme suo.

Passiamo ora ad un carme famoso, e quasi sempre, a parer nostro, male interpretato: il carme XXXXIX a Cicerone:

Disertissime Romuli nepotum Guot sunt quotque fuere, Maree Tulli, Quotyue post aliis erment in annis, Cratias tihi maximas Catullus 
Agit pessimus omnium poeta, Tanto pessimus omnium poeta, Quanto tu optimus omnium patronus.

Auche le cose che Catullo dice in quest' epigramma sono state interpretate generalmente come dette sul serio. E sarebbe molto ameno, per quanto poco istruttivo, esporre le varie spiegazioni tentate.

Secondo il Westphal (1), Catullo ringrazia Cicerone perchè questi, prima amante di Lesbia (2), rinunciando poi a tale amore, areva lasciato libero il posto a Catullo, ed era diventato quindi anche ottimo patrono di lui ! patrono, cioé faroreggiatore di amori? - Secondo il Rettig (3) la gratitudine di Catullo doveva esser per cosa attiuente all' abilità oratoria di Cicerone, per qualche merito che l'oratore si fosse acquistato presso il poeta, in re tamen non ita gravi, aggiunge il critico, il che sarebbe dimostrato sì dall' argomento e dalla natura del carme, che è di genere epigrammatico, sì dalla sua brevità. Un po' di più determina il Pleitner (4): Catullo per qualche suo carme diffamatorio si sarebbe tirato addosso un processo d'ingiuria e sarebbe stato difeso con buon esito da Cicerone! Lo Schwabe (5) pensa che questo epiyramma di ringraziamento a Cicerone sia stato composto per l'orazione pro Caelio, nella quale Cicerone difende

(1) Catulls Gedichte in ihrem geschiehtlichen Zusammenhang übersetnt und erläutert, Breslaw, 1867, p. 238 sgg.

(2) Di Cicerone amante di Lesbia vedi qui appresso.

(3) Rettig, Catulliana I (Ind. lect. Univ. liter. Bernensis, Bernae, 1868), p. 12.

(4) Studien zu Catullus (Prog. der K. Studienanstalt Dillingen fur das Jahr 1876), p. 129.

(5) Schwabe, Quaestiones Catullianae, Gissae, 1862. 
Celio da tutte le aecuse mossegli contro ad istigazione appunto di Clodia, cioè di Leshia, riferendo quindi l'epigramma al 698/56. Il Rostand (1) rimane incerto tra un possibile Pro Catullo d'interesse privato, ed il Pro Caelio, nelle cui invettive contro Clodia il peeta trovava quasi la vendetta dell' infedele. Il Bachrens (2) immaginò che nel $698 / 56$ Cicerone avesse inviato a Catullo, a Verona o a Sirmione, l'orazione per Celio; c Catullo si per soddisfazione del vituperio lanciato eon tanto impeto contro l'infedele, si per gratitudine rerso l'oratore, che avera taciuto il suo nome come amante, rispose con questo epigramma. Egli osserva ehe nou si addice a Catullo la coperta irrisione: egli prende di fronte i suoi nemici. Ma, buon Dio! tutti quelli che dileggiano mediante tirate ironiche prendono di fronte i loro nemiei: se altri interpreta sul serio quelle lodi, che sono invece derisioni, si puo pereio dire ehe le derisioni sieno coperte? Se io prendo sul serio le lodi del Parini al giovin signore, si deve pereio concludere che il Parini deride copertamente? (3)

In rerità non sono pochi quelli che redono nel carme XLIX una tagliente ironia. Citeremo lo Jahn (4), lo Heskamp (5), il Ribbeek (6), il siiss (7), lo Schulze (8),

(1) Eug. Rostand, Vie de Catulle premessa alla traduzione, p. LXX, n. 1 .

(2) Baehrens, Comm. Cat. p. 257.

(3) Credo inutile continuare la serie di yuelli che seguono tale interpretazione.

(4) Prefazione all'ediz. dell' Orator.

(5) De C. I'alerii Catulli vila, Monasteri, MDCCCLXIX, 1. 27.

(6) Valerius Catullus, Eine litt. histor. Skixie, P. 22.

(7) Süss, Catull. 1). 30-31.

(8) Zeitschr. f. d. Oymuasialu. XXX1, p. 700; Roem. Elegie 1. 30 . 
il Benoist (1), il Menozzi (2), lo Harnecker (3), ecc. Ma, anche qui sono naturalmente molto varii $\mathrm{i}$ modi di spiegare le ragioni e l' occasione dell' ironia.

In rerità si stenta a credere come si sien potute credere dette seriamente queste parole, nelle quali Catullo si dichiara il pessimo dei poeti. Anche se supponiamo una stranrdinaria resipiscenza di modestia, un cotal linguaggio non torna: qui non si tratterebbe più di modestia, bensì di umiltà, una virtì, se pure è virtì̀, ignota ai Romani. Il poeta che si scaglia con tanta mordacità violenta contro i cattivi poeti, e chiama gli annali di Volusio cacata carte (c. XXXVI, 1), e chiama Suffeno capraio e zappatore (XXII, 10), e minaccia di odiare di odio Vatiniano l'amico Calvo, perchè gli ha regalato per ischerzo un volume di cattivi poeti (XIV, 1-7), e per vendicarsi vuol comprare i Cesii, gli Aquini, i Suffeni, e siffatti veleni e ricompensare con tal supplizio Calvo (XIV, 16-20); il poeta che chiama empii quei poeti (ivi 7), ed orribile ed esecrando quel volume, che poco mancò non lo facesse crepare (ivi, 12-15), e che finisce la sua scherzosa requisitoria contro gl'infelici versaiuoli col gridar loro (ivi, 21-23) :

Vos hine interea valete, abite,

llluc unde malum pedem attulistis,

Saecli incommoda, pessimi poetae,

(1) Comm. p. 489.

(2) De Catulli carm. XLIX et LXXXXV (Trani, 1895), 1. 6-15.

(3) Qua necessitudine coniunctus fuerit cum Cicerone Catullus (Progr. des Gymn. Friedeberg), Frankfurt a. O., Harnecker u. Co, 1882. 


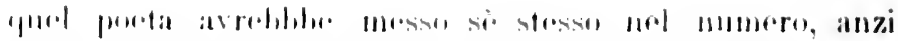

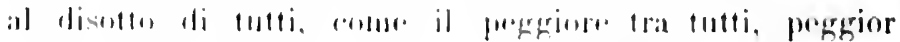

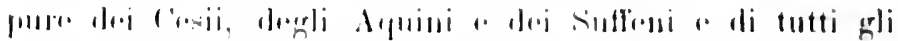

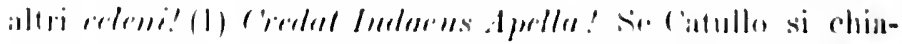

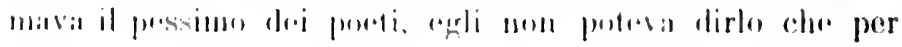

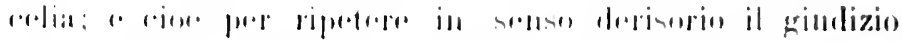

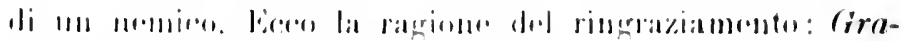

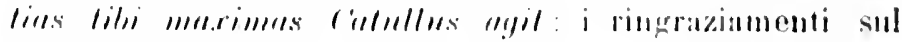

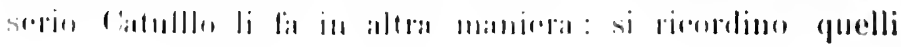
all Allin (I.XYlll," I yre.r.).

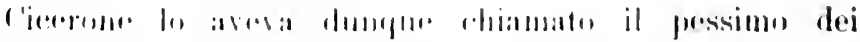

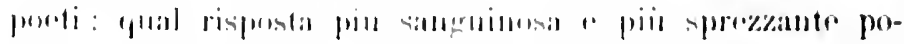

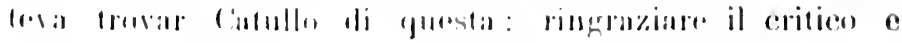

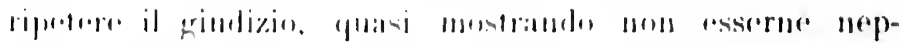

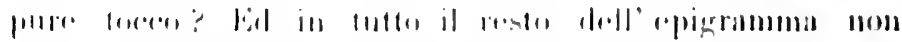

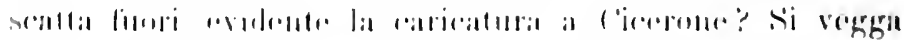

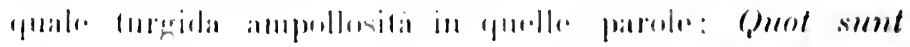

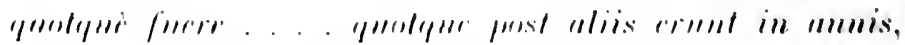

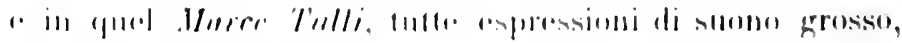
r li lahlura rolialle.

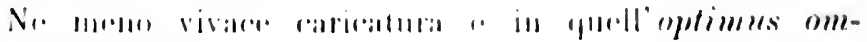
mimm! pullmmls.

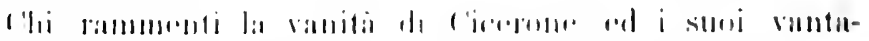

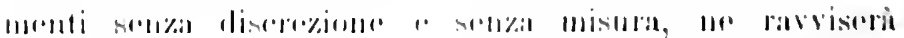

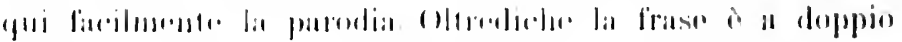

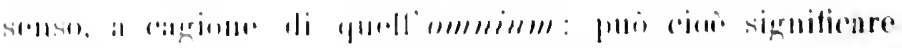

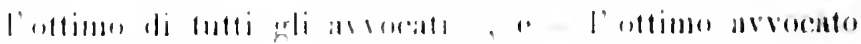

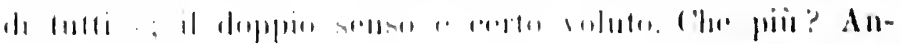

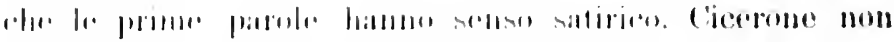

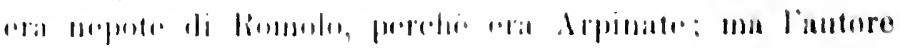


della Declamatio eontro di lui (Ps. Sall. f) Lo chiama Rommle Arprimas: o in rotali apprellazioni di lommle " disertissime lommli nepetum i certe da vedere lat deri-

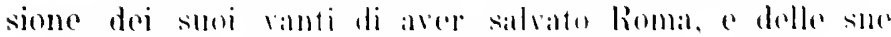

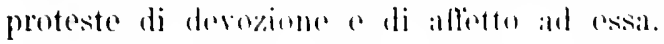

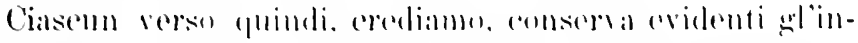
dizii dolla intenzione ironic'a di c'atullo. C'he l'iromia cosi

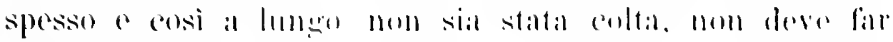

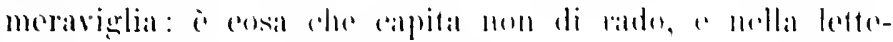
ratura e nella vita. Le punte irencele mon sempere somo

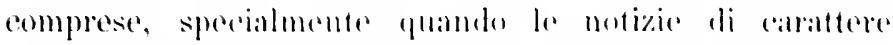
persmale somo searse o mameano aftatto. Di quallte allo-

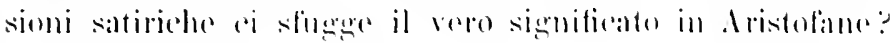
E quante altre non rappremmo, se nom a fossero stati

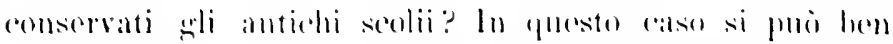

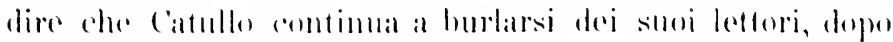

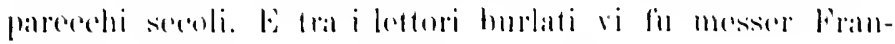

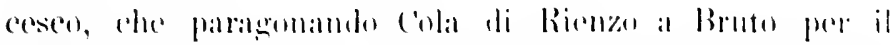

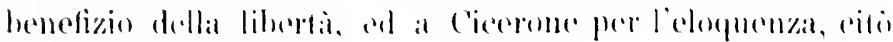
l'epigrammal ('allalliamo (1).

$$
* *
$$

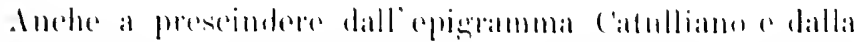

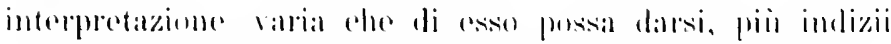

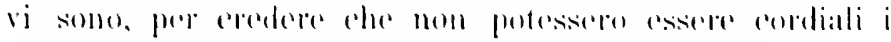

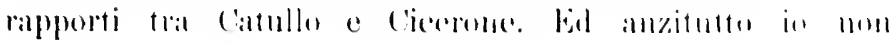

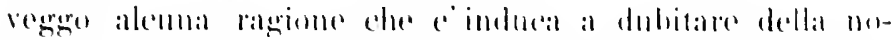

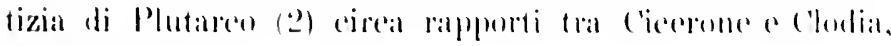

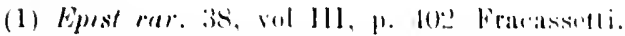

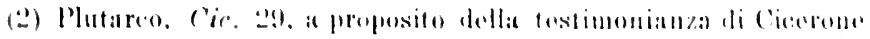

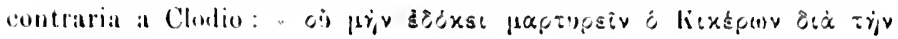


cioè appunto la Leshia di Catullo. Chiunque rammenti le smanie e le gelosie di Catullo e gl'impeti suoi contro i rivali, comprenderà facilmente che pur la semplice voce o il semplice sospetto di tali rapporti, ne doresse aizzare le ire e turbare l'animo.

Dice dunque Plutareo che l'acerbità di Cicerone nel testimoniare contro Clodio non sembrava esser per amore di rerità, ma quasi per iscagionarsi davanti alla moglie Terenzia. (riacchè r' era fra Terenzia e Clodio inimicizia, a cagione della sorella di lui, Clodia. desiderosa di sposarsi a Cicerone, e che areva di ciò aperto trattative a mezzo di certo Tullo, familiarissimo di Cicerone. - Io non istarò certo ad esporre qui tutto il romanzo, che sul fondamento di tali notizie congegno un dotto critico (1): ma d'altra parte neppur credo sia ragionerole pretendere di togliere ad esse in tutto o in parte il loro valore (2). Nell'orazione Pro raelio Cicerone si mostra bene esperto di Clodia: ne parla come di una, della quale egli conosea la vita intima e le disonestì segrete: e mostra saperne molto più che egli non dica, giacchè dichiara che solo quel tanto ei ne dirà che si attenga alla causa (3). E dei

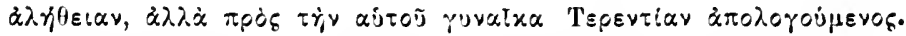

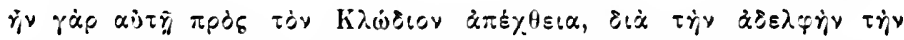

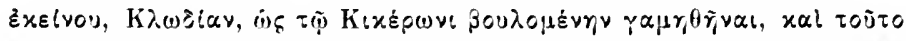

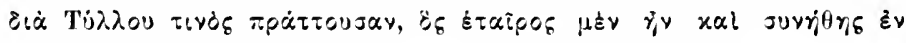

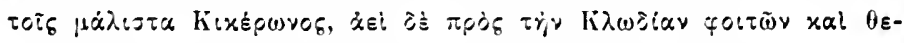

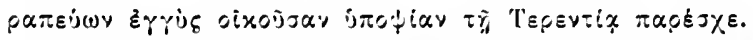

(1) R. Westphal, Catnlls Gedichte in ihrem geschichtlichen Zusammenhange, Breslau, 1867 .

(2) Geppert, Zeitschr. f. d. Ciymuasialu. 1867, 1. 548; Drumann, Käm. Firssh. 11. 1. $212 \mathrm{sg.;}$; Rottig, Catalliana I, p. ti-7.

(3) Pro Carlio, 31: " de qun "g" nilit diram nisi depcllendi criminis causa". 
costumi di quella domna (1), e della sua bellezza (2), e della vita che si srolgeva in quella casa (3), e dei giovani dissipati che la circondarano e rivevano insieme con lei vita di corruzione e lussuria (4), Cicerone parla come uno che ha visto e che dallo spettacolo ha tratto l'energia al suo dire e i colori per le sue vivaci dipinture. L'addurre che l'interesse politico e la dignità personale salvava Cicerone dal disastroso passo, non infirma a dir rero nulla delle notizie Plutarchee, giacchè appunto anche da quelle risulta che Cicerone non abboccò all'amo; l'addurre l' inverisimiglianza che Cicerone per placare Terenzia inveisse contro Clodio è meno ancora conclusivo, giacchè s'intende che quel motivo è riferito da Plutarco come una roce ed in ogni caso è una interpretazione ed una malignazione: la quale però poteva nascere, appunto percho si sapera che Cicerone arera per un certo tempo frequentata la casa di Clodia, e che questa aveva cercato adescarlo.

Cicerone era amicissimo del marito di Clodia, Quinto Metello Celere, e di lui raccolse l'estremo respiro (5). Si può supporre che nel frequentarne la casa aresse aruto occasione di parlare con Clodia del giovane Catullo e di esprimere il giudizio sfavorevolissimo sulla sua arte: indi l'epigramma ironico del poeta, il quale, se mostrava d'infischiarsi del critico, d'altra parte canzonava e dileggiava

(1) Pro Caelio 32, 34, 36, 62.

(2) Ivi, 49.

(3) Ivi, 57 .

(4) Ivi, 67 .

(5) Ivi, 59 . 
in quel bel modo che noi sappiamo, anche il marito (1). Quando Clodia andi ad abitare col fratello sul Palatino, qual meraviglia che cercasse di adescare Cicerone, che ricorresse all opera di un intermediario. Tullo, che Cicerone, ormai a conoscenza di tutto, respingesse i tentativi, e che la sua veemenza contro Clodio e la sorella fosse interpretata dai maligni come mezo per placare i sospetti gelosi di Terenzia:

$$
* *
$$

Del resto anche per criterii di arte era grande divergenza di apprezzamenti e di tendenze tra Cicerone e Catullo. È noto quel che Cicerone sentisse rlei vé́repor. Questi seguivano anche per la composizione e per il ritmo del verso l'arte alessandrina. Catullo fu seguace di quelle norme the gli Alessandrini arevano reso peculiari allesametro. Egli infatti nom ammette la cesura trocaica; egli pone spesso gli spondei in principio dell esametro, usa frequentemente i rersi spondaici, e cioè con lo spondeo al quinto piede, egli chiude i rersi con parole di una, di quattro, di cinque sillabe. Tra queste peculiarita la principale è l’ uso dei rersi spondaici. Catullo amara tanto questa forma che una volta (2) ne adopero tre di seguito. E nell usare il verso spondaico Catullo non sempre allo spondeo del quinto piede fece precedere il dattilo: (3)

(1) C. LXXXIII :

Lesbia mi praesente viro mala phrima dicit:

Haec illi fatuo maxima lavelitia col.

slule, nilial sentis e'cr.

(2) Carme LXIV, is sgg.

(3) Carme IXIV, :3. 
e non sempre chiuse il verso con parole quadrisillabe, secondo l'uso che poi inralse per gli spondaici: spesso chiuse il verso spondaico con trisillabo (1). Ora appunto l' uso di tali versi spondaici era ripreso scherzosamente, come proprio dei poetae novi, da Cicerone in una lettera ad Attico (VII, 2, 1): "ita belle nobis flarit ab Epiro

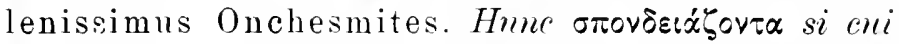

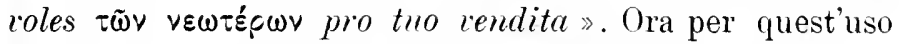

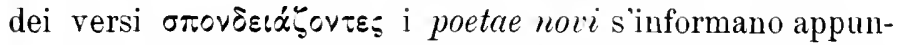
to alla imitazione degli Alessandrini, quali Arato (2), Teocrito (3), Callimaco (4), Apollonio Rodio (5) e specialmente Euforione (6). Si comprende bene come Cicerone contrapponendo Ennio ai poetae nori chiamasse costoro cantores Euphorionis (7). Niun dubbio che tra cotali cantori egli comprendesse nel suo pensiero anche Catullo: e niun dubbio quindi che secondo il suo giudizio artistico Catullo fosse reramente pessimus omnium poeta (8).

(1) Ivi, 44, 74, 96, 252, 292, 297 ; LXV, 23; LXVI. 57.

(2) Vv. 419 ss., 953 ss.

(3) Vv. XV, 29 ss.

(4) In Dian. vv. 222 ss.

(5) IV, 1191 ss.

(6) Cfr. Meinecke, Analecta Alex. p. 62.

(7) Cic. Tusc. III, 19, 45.

(8) Sui poetae novi e sui cantores Fuphorionis v'è tutta una copiosa letteratura. Rammenterò il mio scritto giovanile: Caratteri ed origine della * Nuova poesia" latina nel periodo aureo, Torino, Erm. Loescher, 1890. 

VII.

\section{C'atullo a Verona. \\ l'atullo e la Società romana dell'etì sna.}

La memoria di Catullo, specialmente a Verona e nel Veneto, nell'antichità e nel medio evo. - L'affetto di Catullo per Terona. Catullo a Roma. - Amici e rivali di Catullo a Roma. - I carmi per Giovenzio. - I carmi contro Gellio. - Le poesie amorose per Lesbia. - Cloulia. - Clotio. - Le fasi della grande passione di Catullo. 

Nei primi anni del Rinascimento Italico, verso la fine del secolo $\mathrm{XIII}^{\circ}$, tornava dopo secoli il libretto di Catullo nella sua patria; ed un ammiratore entusiasta del poeta, Benvenuto da Campesano, (morto intorno al 1323), cantara la Resurrexione di Cutullo, de resurrectione Catulli poetae veronensis, un breve epigramma, nel quale con poetica finzione s'introduceva a parlare il libretto stesso reduce, per annunziare: "Torno alla patria esule da lontane terre: un compatriota ha procurato il mio ritorno...) (1).

(1) Nel corlice G, cioè il Parigino 14137, che, come risulta dalla subscriptio del foglio $36 \mathrm{r}$, fu finito di scrivere a Verona il 19 ottobre del 1375, si legge, in principio della detta subscriptio:

Explicit Catnlli Veronensis libellus (interstizio)

Versus domini Benevenuti de Campexanis de Vicencia de resurectione Catulli poete Veronensis:

All patriam venio longis à tinibus exul:

Causa mei reditus compatriota fuit.

Soilicet a erlamis, tribuit cui francir nomen,

Qnique notat turbe pretereuntis iter.

Quo licet ingenio vestrum colebrate tratullum,

Cuius sub noslio clansa papirns erat. 
Tornava dopo secoli alla diletta Verona, il cui nome non si scompagnò mai da quello del suo poeta, giacchè C'atullus Veronensis fu appellazione tradizionale e costante, continuata dall' età classica al medio ovo ed al rinascimento (1); a Verona, dove non era mai venuto meno i] ricordo ed il culto per il figlio glorioso; sicchè anche in

Questo epigramma si trova altresi in molti altri codici, in fine $o$, più raramente, in principio del lihretto catulliano; li puoi vedere notati nella edizione dello Schwabe, p. 101 ed in quella di Robinson Ellis, 1. 1. All'epigramma segue la fino lella subseriptio, we lo scriptor chiede scusa al lettore degli errori, esprime la speranza che si ritrovi un esemplare piu corretto e pone la data sopri detta. - Su Benvenuto da Campesano Vicentino e sulla interpretazione dell' epigramma redi Robinson Ellis, I'rolegomena alla sua ediz. p. XII-XV. Nol v. 3 si accema forse al nome dell'inventor, che può essere stato Francistus de Calamis. Anche difficile è l'interpretazione del v. 4 ; cfr. lo Ellis. Si tratterà forse di un Franciscus Cursus do Calamis? Nel r. (j modius vuolsi traslato da Luca XJ, 33 (Haupt, Quarst. 1. 8); " quamquam in tam $\alpha i v(\gamma \mu \alpha \tau() \geqslant \varepsilon t$ carmine non adeo certa est sententil ut non vere sub horreo aliquo liber reperiri potuerit , (Ellis, p. XIV).

(1) Catulli veronensis liber incipit è la inseriptio del cod. Sangermanese Parigi 14137), e li quasi tutti gli altri codici, aleuni dei quali argiungono veronensis poetae illustris. La subseripto ir Explicit Catulli veronensis libellus, nel Sangermanese, e cosi a un dipresso negli altri. - Cfr. Ovid. Amor. III, 15, 7 a Montua Vergilio yaudet, Verome C'atullo «; Plin. X. II. 36, t5 * Catulli Veromensis "; Harziale I, 61, 1 "Verona docti syllabas amat ratis s; 14, 195 magnu suo debet Ferona Catullo ; Surtonio, in S. lierol. Chron. ad. ann. Abraham. 1930) (=6678 di R.) "(iaius Ialerives Catullas seriptor lyricus Jeronae nascitur" ; Nonio Marcello 546, 26 a Catullus l'erone'nsis , Guglielmo Pastrengico,

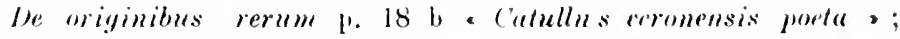

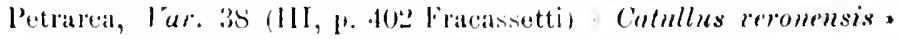
(nosi anche in sien. 11, 3, p. 97s). 
tempi di fitta barharie, il Vescovo Raterio di quella città, del secolo $\mathrm{X}^{n}$, si rantara di poter leggere Catullo, giammai letto da alcuno per lo innanzi (1). E con che festa fu accolto subito in Italia il risorto Poeta! Tosto da lui tolse fiori di poetici luoghi per un suo compendio morale Geremia griudice di Montagnone, padorano, morto poi intorno all'anno $1300(\stackrel{2}{)}$ : ed un altro padovano, Albertino Mussato, si ispirò eridentemente in più di un luogo alla imitazione Catulliana (3); ed a Verona un ignoto raceo-

(1) Raterio, vescovo di Verona. in un sermone tenuto a Verona nell'anno 965 (p. 639 ed. Baller:in.): "Qnid de me dicere, quid raleo cogitare, et ut turpia subsilens, honesta solmm, prohibita licet, depromam. si in leye dei, ut (cuius?) debitorem me fore non nescio, die rion meditor ae nocte. Catullum mumquam antca lectum, Plautum iam olim lego negleetum, musieam quando saepe rogatus: cxpono ». Neglectum (per nee lectum) è nostra congettura; efr. Bollctt. di filol. class. XII, 1906. -- Un accenno al carme LV, 16, 17 è forse in Raterio, p. 621, B. ed. Ballerini. Altri accenni addita lo Ellis nei Prolegomena alla sua ediz. di Catullo, p. VIIIIX; ma aggiunge: "Quamquam non infitior ne umum quidem locum ex lis tam clare ad Catullum referri ut non aliunde derivari potuerit .

(2) Ieremias iudex de Montagnone, Compendii moralium notabilium part. I, lib. 3: Cat. XXII, I8; II, 1 : Cat. I.XVIII,b 137 ; III, 4: Cat. LI, 15; IV, 4: Cat. XXXIX, 16; IY, 5: Cat. IXXVI, 13; IV, 6: Cat. LXVI, 15. Chr. la ediz. dello Schwahe, 1). XIV (Testimonia, n. 61). Questi el altri luoghi addusse lo Ellis, Prolegom. citati, p. X-XI, altri da sè trovati, altri indicatigli da Ingram Byrater. Sulle piu antiche citazioni umanistiche da Catullo efr. Ullman, in Classical Philolny?, V, 1.

(3) Lo Sehwabe (ediz. p. XIV, Testimonia, 1. 62) cita un rarme seritto nellanno 1314, (p.39 ediz. Pignori), in cui si legge: 
Elitore usufruiva qualche rerso di Catullo per una sua antulogia di autorità morali (1), e fruglielmo Pastrengico Veronese, più rolte nell' opera sua sulle origimi delle cose troviva modo di menzionare il suo glorioso concittadino (2).

$\grave{E}$ evidentemente un'allusione al passero. E cita un altro passo (1. 3) Pign.): "nemo tam fortis ralet esse que non fortior assit », efr. Catullo LXVI, 28. Per il seconto passo lo Ellis ̀̀ in lubbio (Irolegg. p. XIII. n. 3): per il primo propende alla opinione dello Haupt. che il Mussato abbia preso la Marziale VII, 14 \& XIV, 77. Quel primo carme è dell'anno 1:314: in quell'anno infatti il Mussato ringraziò con quellia elegia l'Accademia Patavina della corona aurea donatagli.

(1) Schwabe, Testim. nello edliz. 1. 63: a flores moralium aurtoritatum a. 1329 Veronae ab homine ignoto collecti lib. 2 c. 3 ' $d c$ errore' (Catullus ad Varum): Calt. XXII, 19. Vedi di ciò Sehwahe. Ine rorlicibus arrhetypo et reronensi Cutullianis, Dorpati, 1865, p. 11. nota 13. Questo paseo del carme XXIl era stato citato anche da Geremia le Montegnone, ma si per la diversita dolla lezione, si perehe in Geremia manca il titolo Catullus ad Varum lo Ellis (Iroleg. eitati p. Xl) concluse the il racoglitore prendesse dirctamente dal codice catulliano.

(2) Una volta (De originibus rerum 1. 16 a el. ven.) dice semHicemente: reronensis pocta, riferendosi al carme 1, 5; un' altra volt a ( $k .1 \mathrm{~s}^{\mathrm{b}}$ ) it nomina: * Cotullus, reronensis pocta, Ciceronis coetrueus, librum rario metrorum genere exaratum, multa iocosa et placita rontinentr'm. scholusticis lryendum tradidit Frothotomaci Alexandri temporilmss". - Ina terza citaziono è errata (p. 850): - addiditque Catullus rum (sc. Mammram) primum tot is acdibus

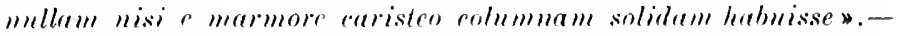
la notizia i invere in Plinio, II. X. XXXv1, 4S. Plinio ora considerate come regonese. come risulta dit aldoni passi del Potralea (1.fr. I). Volhar, Prilrerque et l' humunisme. II, 69 n.); si può congetturare che cigulielmo lastrengico abhia preso la eitazione da u11 passo li serittore anturiore, nel quale Plinio era indicato como

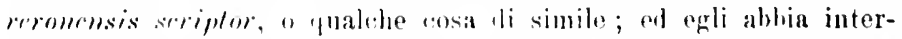

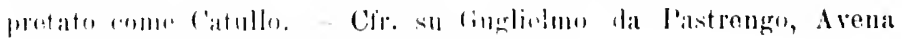

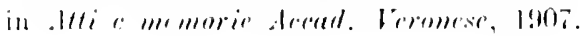


La fama se ne spandera oltre i termini della città e della regione. Francesco Petrarca era un grande ammiratore di Catullo (1), ed in più luoghi imitatore (2); e Giorami Boccaccio nell' opera sua sulle Genealogie degli Dei in-

(1) 亡े notevole, osserva il De Nolhac (Pétrarque et l'hum.2 p. 165), che i poeti erotici, per quanto ciò possa parere strano, ispiravano al Petrarca una certa repuguanza (cfr. Rem. I, 69); se egli citò spesso Catullo, ruol dire che le sue poesie gli erano presenti allo spirito: da ciò le imitazioni, forse inconscie reminiscenze. - Circa la scoperta del manoscritto catulliano, a Padova o a Verona, alla fine del XIII secolo, efr. Robinson Ellis, Catullus in the XIV Century, London 1905, p. 21-23. Secondo W. Gardner Hale (Classical heriew, vol. XX, p. 160-164) il codice catulliano del Petrarca era l'Oxoniensis $(=0)$, pervenuto alla Biblioteca Bodleiana dalla collezione Canonici di Venezia, e che ogli credo anteriore al famoso Sangermanensis. Il Camoniciano (Oxoniensis, O) è 11 embranaceo, di fogli 37 , in $t^{0} \mathrm{gr}$; cfr. Henricus 1). Coxe, (atalogi cod. manuseriptorum Bibliotheeae Bodlcianae, pars III, $10 \mathrm{xo-}$ nii, 1864), p. 124, num. 30. - Nel famoso codice vergiliano del Petrarca, ora all'Ambrosiana, si trovano più volte menzioni di Catullo, efr. i fogli 8, 28, 29, 52, 56, 108, 113, 114 ; vedile riportate in De Nolbac, Pétrarque et l'hum. 2, Paris, 1907. I, 1. 167-168. Alcune volte il nome è erroneamente scritto Catulus(f. 8 e 114). L'epitalamio di Peleo e Teti è chiamato Peplon, certo per la descrizione del drappo nuziale (f. 52 e 108). Altre citazioni e menzioni sono suarse qua e là in molte cpere del Petrarca; cfr. fra i Testimonia della edizione Schwabe quelli raccolti sotto il $n .65$ (p. XV), e i citati Prolegomena dello Ellis, p. XVJI-XVIIJ.

(2) Le imitazioni e le reminiscenze da Catullo nelle opere italiane e latine del Petrarca furono raccolte dal De Nolhac, Pétrarque et l'hum. ${ }^{2}$ : 1, p. 169-171. - Lo Schwabe, op. cit. p. XV, che sembra essere stato ignorato dal De Nolhac, notò: Epist. Fam. 24, 5 $1=$ p. 272 Fracass.) "lethaei guryitis unda non obruit » cfr. Cat. LXV. 5 "lethoeo guryite. . alluit unda pedem * Sonetti. I, 49: "Non preyo gia, nè puote arer più luoeo, Che misuratamcnte il mio cuor arda, Ma che sua parte abbia costei del fuoco»; cfr. Cat. IXXVI, 23-24: * Non iam illud quaero, contra ut me diligat illa, Aut, quod non potis est, esse pudiea velit... V. anche i citati Irolegomena dello Ellis, p. XVII-XVIII. 
troduce un vivace quadretto di compagnie galanti, nel quale abbiamo altresi mottestazione del gran favore che già godera il Poeta. Egli ci rappresenta gli zerbinotti amanti di arrenture, che mandano lettere di amore a domette sorridenti, e compongono versi e canzoni, e srol(x)no per comporli, i rolumi di Catullo, di Properzio, di Ovidio (1). Poi quando dalle grazie e dagli adescamenti di quelle poesie sono allettati e rinti. si volgono ad accusare quei loro maestri come seduttori degli animi loro, mentre sono stati proprio essi, di loro spontanea volonti, a cercarli.

E del farore con cui Catullo era studiato ed amato fanno fede e le ripetute insistenze di Colnceio Salutati forentino al veronese Gaspare de Broaspinis e ad altri per averne un esemplare (:2), ed un earme di Antonio

(1) La frase del Boccaccio (Gen. Mror. XIV, 16): " Ilinc Catulli. Propertii et Vasomis rolmmina erolmut o is stata variamente wiudicata. L'Hortis whelii sul Boce. 11. 944) vi seorse la prova irlla diffusione, nel see. XIV, dei tre autori; il De Nolhac, I $i$ -

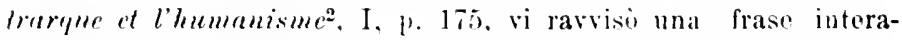
mento oratoria, dalla fuale non fosse possibile trarre alcuna conalusione. Ma questa interpretazione sembra eceessiva, anche so si ruole seorgere pualche esagerazione nelle parole del boceaceio.

(2) Lettera 16 Nov. 1:375 di Coluceio Salutati al veronese Broaspini, cfr. Norati, Enistolario di Coluecio Sal.. Tomo I, Roma. 1891. 1. 22) s.r.: " ratullum quem credo furrmm libellum ant rxemplatum ant exemplandum rogn transmitle. Tement ibi Flosentimi. qui totum trrarmm orbem discmerndo terunt. pro merribus apotheress : in ballis quas fuciunt illum iubere poteris alligari. quem ad me. "nt arbitere, libenter quilibet destimabit . E cosi in altre letfore allo stesso. Già prima, il 25 Lugrlio 1374 , Coluceio avera suritto a tal line a benvomuto la Imola, ed a proposito di

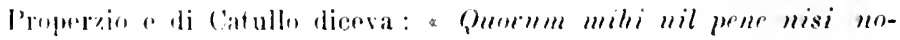
men inmotuit (Novali, op. eit. [1. 170). 
Beccadelli, detto il Panormita, che insieme ci mostra come altresi le colte gentildonne fossero prese da passione per il poeta: "Ardo, Galeazzo mio, dice il Panormita, del desiderio di ritrorare il molle Catullo, per potere obbedire a colei, che i padrona del mio cuore. Ella, che i voluttuosa, ama leggere i teneri poeti e preferisce i versi tuoi, o dotto Catullo. E testè, carezzerole, con molte preghiere mi chiese il sno poeta, credendo ch' io l'aressi. Son posseggo, risposi, luce mia, ninfa mia, questo libretto: ma lo procurerò; forse arrai l'opera. Ella insiste ed a qualunque costo ruole il libro che le è caro; e mi tratta con dure minacce. Laonde, per tutti gli Déi, io ti preğo, o ciro amico, (e possa cosi Citerèa essere propizia ai roti tuoi !), ti prego, cercami questo libretto, affinchè io torni più gradito alla mia Dea »(1). Di lì a non molti anni (1450-1470) Battista Guarini figlio, Veronese, spese amorose cure per mondar Catullo dei non pochi crrori che l’ignoranza dei copisti vi areva accumulato (2), e fueenrlo

(1) Antonii Beccatelli Panormitae Hermaphroditus, 2, 2:; 1. 1:28 ed. Forberg. Il poema fu noto nel 1426. Vedi nello sulhwahe, "p. cit. n. 73 , P. XVII, alcune imitazioni e reminiscenze catulliane nell'Ermafrodito. Sull Ermafrodito serisse una epistola Guarino Veronese, pubblicata piu volte; v. ora Sabbadini, Eyistol. di Guarino Yer. 1915, p. 505 e $70 \%$; ed in essa, come in parecehi altri luoghi delle epistole o di attre opere, Guarino addusse versi catulliani.

(2) Cfr. Schwabe, Index scholarum Dorpat. 1865, p. 10. Fu spesse volte confuso Guarino padre col figlio. All' uno e all' altro fu attribuito un lavoro di emendazione lel libretto catulliauo. Anche una epistola datata da Ferrara VII Kal. Aug, 1456. nella quale si parla di tal lavoro, fu attribuita al padre e al figlio. Fn exlita da Eug. Abel in 7ritseh. fiir nester. (iymn. XXXIV, 161; e su di essa v. R. Sabbadini. Rin. filol. XIII, 266: XIV, 179. Nolla epistola si legge: "Catullum ubi meliorem fecero, ad proprios 
domo alla patria Verona dellesemplare emendato, rivolgeva allantica non immemore cittì un carme, che così finiva:

(1) s che verria fuori un Catullo nitido e terso: cotali doni ti famno i cittalini tuoi: (1).

$$
*^{*} *
$$

Catullo non era daremo immeriterolf dell affetto maternu, onsle Verona prosexul per secoli la memoria sua (’2).

lures remeare enmpellom ". che sembean areordarsi eol carme di Battista (iutrini figlio, di eni riporteremo ni ultimi versi nella nota semente.

(1) Cili ultimi versi del carme sono:

Barbarion ritu quidam forch lowgutum,

Tantue illormu guimis obstiterant touncorat.

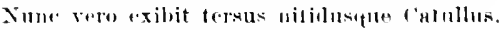

Haec tili dant wives munera grata tni.

Cfr. Selhwabe op. cit. 1. XX. - A cagrone appunte di questo vanto del Guarini, di ridonare cios alla patria il suo Catulle, al"mui attriluirono anche al hiuarini l'antion eligramma di Benvenuto da Cammesano sopra riportato : eosi nei codici parigrini 7990 , s.:3e, e nelle antiche edirioni.

Di altri due reneti hastorà far semplice menzimne, cioce Siceo I'olutone, padovano ( $\rightarrow 1463$, we nella fipitome in scriptormm illustrimm lotimne lingnere ritas serritta nel 1438, introdusse una hrore biogradia di Catullo Iratta dei carmi stensi del poeta credila prenso lo lillis, op. rit. p. XIX desuntat dal codice Harl. 4i69); o ryuthies detto cenetensis, hal nome di una horwata presso Tre-

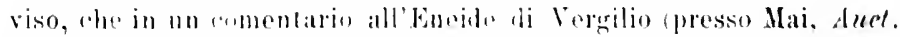

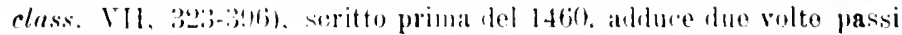
di Catullo.

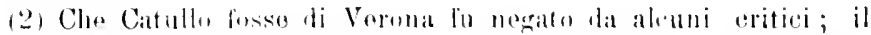

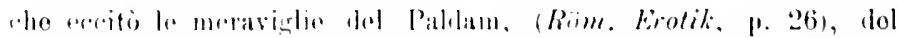

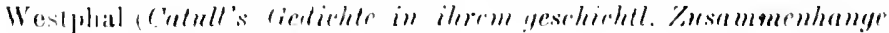


Iri era stata la sua casa paterna, ivi egli avera riceruto la prima educazione ed avera acquistato il gusto per la poesia; ivi avera passato giocondamente alcuni anni di spensierata giovinezza, e ne avera lasciato ricordo in uno dei suoi carmi più belli (LX VIII a, 15-18), ivi era tornato dopo le amare delusioni, e vi areva passato giorni

Bresl. 1867, p. 44), dello Schwabe (Qu. Catull. Gissae 186:), 1. 28) e d'altri, i quali stettero fermi all'aturità di S. Gorolamo, ohe scrive (ad Olymp. 173, '2): *C. Valerius Catullus seriptor lyrivus Veronae nascitur. Ma usservo lo Hestamp, De C. Valerii Catulli vita ecc. Monasterii, 1869, p. 8) whe S. Gerolamo dice di Ennio (ad olymu. 135, 2) * Q. Ennius poeta Tarenti nuscitur क, mentre da un verso stesso superstite di Ennio risulta che egli era rudino. E per quanto riguarda la menzione di Verona come patria di Catullo fatta da Uvidio (Amores III, 15, 7) e Marziale (XIV, 195), dice lo Heskamp: "quod illi viri summae anctoritatis I crgilium Mantuae natum esse dieunt, quumquam satis constut illum. in pago quae Andes rocabantur esse ortum, quid retat in Catulli quoque loco natali definiendo nos ab illis deecdere? Ios erat apnd seriptores antiquos ut urbem saepe pro rilla aut pago proximo ponerent ». Lo Heskamp ritiene dunque che egli fosse di Sirmione, e ciò vede indicato nel larem ad nostrum della poesia su quella pupilla delle penisole (XXXV, 9). Senonchè bisogna tener conto di altri fatti, che rendono inconcussa la tradizione di Verona come patria del poeta. L'espressione Feronae meac di LXVII 34 è abbastanza significativa. Quando Catullo vuol mandare un invito a Cecilio, lo invita appunto a Verona (XXXV, 3. E a Verona eyli si ritirò per qualche tempo dopo le turbolenze e i disinganni della vita romana (LXVIII, 27). Ciò sembra indicare che apprinto a Verona fosse la sua faniglia e la sua casa. Le iscrizioni ci attestano come a Verona continuasse la famiglia dei Valerii (Schwabe, ( $u$. Cat. p. 27); un Valerio fu creato a Verona llamine di Augusto e pretore nel 776. - Circa il soggiorno dol poeta a Verona ctr. Auguste Conat, Etude sur Catulle (Paris, Thorin, 1875), p. 20-:34: Catulle d̀ Vérone. 
tormentati dalla grelosia, mentre lamico Manlio lo arrertiva che aitri a Roma rubara nellabbandonato letto il posto suo (LXVH a, 27-30), ivi egli avera invitato l'amico Cecilio a passar seco qualche ginmo di ristoro, sciogliendusi dai lacei li una passione (XXXV. 3) e tutto il tenero affetto sul) verso la patria egli avera dato a divedere qua e la in quelle espressioni : la mia Verona (LXVII, 34), $i$ mici Tramspadani(XXXIX.16), che sono tanto piu significative, quanto piu spontanee. Il grido straziante di dolore per la morte del fratello, e la rappresentazione, che qua e là ritorna, di teneri affetti domestici, ci rivela nel sriovane Catullo una edncazione li antica sanita morale, ma consuetudine di famisclia amorosa ed omorata (1). Ma hen presto si fisso a Romal, la gran corruttrice. Fu certo qual-

(1) In un lreve tratto dol carme LXVIII a (v. 15-18 orli aecenna alla sua vita di serlici anni, passata tra i piaceri della posia c degli amori. Che a questo primo periorlo della sua grovineza lebbano ascriversi alcune poesie, w' è ricordo di focosi o lacili amori (XXXII), li vosilerazoni maligue sopra un marito veronese (XVII), o lelle indiscrezioni ehe il preta gioeosamente fa rivolare alla iamua della rasa di Corilin (I.XFIl), fu pwisiero di

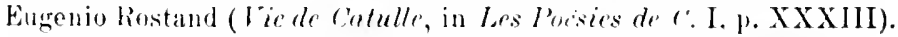
Sutiano cho nel carmo XVll l'espressivne municipem meum (v. S) i) piit naturale in hoosa a Carullo. fuablu si supponera la poosia seritta a lema: ivi infatti futovi venimeli il pensere di rotareche quel tale ora un suo complasamo. E pere quanto riguarda il carme

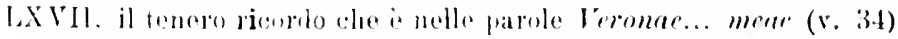
moglio si comprende, smpromondo il peeta lontano da Veroua. \& la pii falto det viageialori, montro viagerano, somo amanti del loro

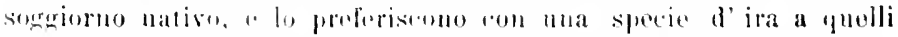

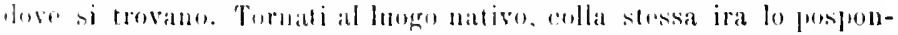

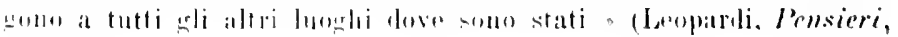
XXXi. 
che anno prima del 689, e Catullo avera allora poco meno di 20 anni (1). Nella società frivola, corrotta, e galante di Roma si andarono cancellando le antiche virtù semplici e modeste della sua Terona. Egli amò l 'inebbriamento, il turbine della vita romana. Conobbe tutti i vizi e tutti i piaceri, gettandosi nelle arventure del gran mondo: volle far pompa di ville (2) e di amorazzi, tenere concubine ed amasii, e contenderli a più ricchi e più fortunati aspiranti (3); mettersi al seguito di un pretore per tentare di impinguare la sua borsa, mediante

(1) La poesia per le nozze di Manlio e Giunia Aurunculeia (c. LXI) fu certo scritta a Roma; e se due famiglie nobili di Roma accettarono per cantore il giovane poeta, ciò vuol dire che egli vi dimorava da qualche tempo e vi aveva acquistato rinomanza. Ora Giunia mori rerso il 690: si può dunque porre aleuni anni prima di tale epoca l'arrivo a Roma del poeta, e cioè nel 6860 687. I termini della vita di Catullo dati da S. Gerolamo sono dal 667 al 697. Però siccome dal libro stesso di Catullo risultano accenni a fatti posteriori al 699, questi termini han dato luogo a molte discussioni. Forse S. Gerolamo trovò nella sua fonte che Catullo morì di trenta anni (S. Gerol. ad a. 697: Catuilus trigesimo aetatis anno Romae moritur) e non pensando trattarsi di un numero tondo o approssimativo, fissò al 697 la morte. Lo Sebwabe suppone $\mathrm{i}$ termini dal 667 al 700 (efr. Teuffel-Schwabe, Litt. $^{5}$, I, p. 444, 2). Lo Schmidt 672-702 (cfr. il suo articolo polemico in Rheinisches .14nseum LIX, 1914, p. 267-283).

(2) Della villa di Sirmione parla (c. XXXI) con tenerezza simpatica e con l'animo antico; sì però parla con spavalda boria e bramosia di ostentazione nobilesca della sua villa sabina o tiburtiua (c. XLIV), e deride le ville altrui: quella di Furio, carica di ipoteche (XXVI), ed i fondi sterminati di Mentula (CXIV, CXV), che non salvano il padrone dall'indigenza.

(3) V. ad esempio i carmi XV, XXI, XXIV, XII, XIVIII, LXXXI, XCIX, CX, CXI. 
depredazioni in lontani paesi (1); lanciarsi nei pettegolezzi politici e mondani (2): assumere il tono e il linguaggio di tutti i giovinastri della nobiltà romana, cui era titolo di superiorità e di orgoglio l'ostentazione sfacciata dei vizi (3).

(1) Cfr. i carmi X e XXVIII. La spedizione di Memmio avvenne al cominciare del 697. La coloors praetoria era formata di giovani che come segretarii, giuristi, fornitori, tecnici, collettori di vettovaglie, poeti, circondivano il pretore, formavano il suo consiglio privato, si assidevano alla sua mensa, lo aiutavano nei suoi lavori.

(2) Cfr. gli accenni alle labbra candide di Gellio (LXXX), el ai suoi incesti (LXXXVIII, XC, XCI), alla hocea di Vettio (XCVIII), erl a quella di Emilio (XCVII), ed ai denti di Egnazio (XXXVII e XXXIX), ed agli amasii di Cesare (LIV), ed a Mamurra (XXIX e IVII), ed ai duemila amanti di Mucilla (CXIII), ed a Rufa bolognese fellatrice e rubacchiatrice di cene funebri (LIX), e al caprone sotto le ascelle di Rufo (LXIX), ed a Gallo mezzano di turpi amori (LXXV!II), ed a Vibenna, che non trova da vendere le sue natiche (XXXIII) ece. Per quanto riguarda l' apprezzamento morale dei tompi, non sarebbe giusto prendere troppo alla lettera le informazioni catulliane. E la prova se ne ha in Catullo stesso. Per aver parlato (nel carme VII) delle parecchie migliaia di baci che egli voleva dare a Lesbia, Aurelio e Furio andavano spargendo che Catullo non fosse buon maschio, il che provoco nel carme XVI (vv. 12-14) le oscene risposte del poeta. Tanto era pettegola e maligua la società in cui Catullo viveva!

(3) Cfr. XV, 17-15: \& patente porta Percurrent raphanique mugilesque "; XVI, 1 e 14: " Pélicaluo ego ros el inrumabo s; XXI, 13: "Te finem facias set inmumatus ; XXVIII, 10 : "1) Uemmi, bene me a" dan snpimm. Tota ista trabe lentus iurumasti : XXXVII, 8: . Ye une theentos inrumare sessoress. - Circa le relazioni di Catullo col patriziato, efr. Budinger Hax, Catull und der I'ntriviat. eine historisehe Untersuchung (Sit:-here der philosophisch-histor. Clawse der liais. Ahad. der Wissemschaften 121, (ts90), Wien, (F. Temprky), \$. 4 e 5 ; Au-

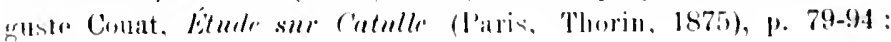
c'alulle al la Voblesse lirmuaine. 
L'antico provinciale parla ormai della provincia con tono altezzoso; e se vuole abbassare in confronto a Lesbia una vantata bellezza femminle, le dice: «E la prorincia trova che tu sei bella ed osa paragonarti alla nostra Lesbia?" (XLIII, $6-7$ ).

Egli è il gran siguore della capitale, e conosce ormai le bellezze fini, le vere bellezze; e come appunto i giovani che fanno vana ostentazione di nobiltà e di ricchezze, si cruccia che una sua villa posta tra i territori della Sabina e di Tivoli, possa essere chiamata Sabina piuttosto che Tiburtina: quelli che la chiamano a qualunque costo Sabina sono i suoi nemici, che vogliono offenderlo! X LIV 2-4).

$$
*^{* * *}
$$

Della dissipata ed oziosa società nobilesca di Roma, questo giovane, naturalmente buono, ma cedevole alle tentazioni ed alle seduzioni, assume, come abbiamo accenuato, il tono di spavalderia cinica, di superiorità boriosa, di ostentazione beffarda della propria forza fisica o sessuale, o delle proprie ricchezze, di lubriche minacce contro chi osi stargli a fronte o tradirlo. A poco a poco la consuetudine con tanti giovani di corrottissima vita par che ottunda nell' animo suo ogni senso di nativa onestà, sicchè pur rappresentando con insistenza e quasi con compiacenza il maggior fango umano, raramente ha scatti o impeti di protesta o di sdegno. Egli si scagliava contro Mamurra, perche dopo avere dissipato i beni paterni aveva divorato la preda pontica e l'iberica, ed or tendeva allia gallica e alla britannica: e rivolto a Pompeo e a Cesare gridava loro: "l'er costui dunque, o genero a suocero, 
avete mandato tutto in rovina? 》 (XXIX). Ma egli stesso, mettendosi al seguito di Memmio, non altro avera sperato che li far grossi bottini: mentre Memmio, furbacchione, avera pensato a sè e non alla coorte; ed ecco Catullo a scagliarglisi contro (X, 12: XXVIII, 4-10), ed a compiangere Veranio e Fabullo, cui era toccata pari sorte (c. XXVIII); era dunque inutile mettersi al seguito di nobili amici : "Pete nobiles amicos!" consiglia in tonn ironico il poeta (XXVIII).

Questi giovani dati al lusso ed alla vita galante, pure se ricchi (e ricco era infatti Catullo) (22). si trovarano spesso, per le loro dissipatezze a corto di quattrini, e Catullo invitando l'amico Fabullo lo arverte di portarsi egrli stesso la cena, giacche la sua borsa è piena di ragnateli (c. XIII). Ma altrove il poeta ci rappresenta i due amici Veranio e Fabullo addirittura come due ignobili parassiti, i quali stamo nel trivio ad aspettare che passi qualcuno che li inviti a cena: ni di tale ultima abbiezione eyli si sdegna, bensi solo si antira che il ricco Pisone

(1) Bene il Rostand, Vie de C. premessa alta ed., p. XLI : "Tout indique au contraire que Cutulle fut laruement doué du cóté Jes arantages matériels. On en peut juger ì ce qu'il nous laisso voir de ses relations avec les nobles et les chevaliers, de ses gouts, le ses habitules, dans cette existence ruineuse, où les recherches cligantes sont de mise en tont (XXV), oi on fait assant de dissifation et le dipense, oi on paye 10,000 sesterees - environ 2000 francs - les services d'un entremettour (CIII), où les meilleurs en virnnent, le patrimoine epuisé par des emprunts à taux énormes, juspu'an metier de parasites is l'alfit d'invitation (XLVIl). Catulle noms anprend in outre gu'il est proprietaire de donx habitations de campagne, l'un sur la frontion de la Sabine, l'autre sur les

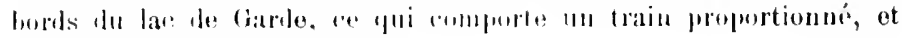
implingue plus que l'aisamere. 
preferisca altri due, affamati, "scabies famesque mundi», al suo Veraniuccio ed a Fabullo (c. XLVII). Ma quando si tratta di pigliarsela coi shoi nemici o coi suoi rivali, o di deprezzarli agli occhi delle amanti o degli amasii, allora non più queste franche, giocose dichiarazioni di trovarsi a seceo, bensì la boria altezzosa dell' aristocratico, che si fonda sui beni di fortuna per credersi superiore agli altri, e rimprovera altrui la povertà. Per la porertà, ad esempio, se la piglia con Furio; la villetta di Furio era bensì al riparo dei venti, ma era esposta alle ipoteche, per quindicimila e dnecento sesterzi, e quello sì che era un rento terribile e pestilenziale (XXVI); erf in due carmi (XXIII e XXIV) insiste nel notare che Furio non ha un servo e non ha forziere; eppure (iiovenzio si è invaghito di lui, e gli soggiace. Ed Aurelio? Egli è il padre di tutti gli affamati, che furono, sono o saranno mai; eppure tenta insidiargli l'amor suo (c. XXI).

Quando non è fortunato negli amori o neyli amorazzi diventa inplacabile contro i rivali più fortunati di lui. Quel tale Giovenzio, il fiore dei Giovenzii, dovette farlo molto soffrire: nè valeva decautarne gli occhi dolci come il miele (mellitos oculos, c. XLVIII, nè valeva rapirgli i baci (carme XCIX); ed ecco Catullo ad inveire contro i preferiti, e nou solo contro Furio che almeno era bello (XXIV, 7) ma altresì contro quell' altro amante, nato a Pesaro, paese di malaria, e che è nell'aspetto più pallido di una statua indorata, e che Giovenzio osa preferigli, e non sa qual triste fatto sia il suo! (carme LXXXI). E così, contro tutti i suoi rivali e i suoi nemici egli trova modo di avventare le sue saette, o rappresentandoli sotto gli aspetti ridicoli, o bollandoli con marchio d' infamia: Emilio fa il bellimbusto. e concuista femmine; eppure ha 
la bocea puzzolente farme XCTII : a peggio ancora il putido Vettio: se vuol far crepare qualcumo, hasta che egli apra la bocca. e tutto é fatto (carme XCYIII): Aufilena, che gli ha frodato la mercede pattuita, perche mon grli ha dato ciò che areva promesso, (carme CX), preferisce poi grenerare fratelli dallo zio (carme CXI): Rufo, che egli inrano credette amico, Rufo, il veleno crulele della sua vita (carme LXXVI), ha il caprone sotto le ascelle (carme LXIX): (iellio, il terrihile (iellio. per cui il poeta avera raccolto i carni di Callimaco, sperando renderlo piì mite e benevolo, ed evitarne gli strali (carme CXVI), Gellio è rappresentato come un mostro, le cui labbra sono stillanti per vizi orrendi (carme LXXX), un mostro che ì magro e sottile, perchè si sciupa negli incesti con la florida madre, con la graziosa sorella, col leguadro zio, con tutte le giovani parenti. di che è piena la casa (carme [XXXIX), dal sno tremendo amplesso colla madre deve nascere IIn mago (carme $\mathrm{XC}$ ): le sue colpe non laverebbe l'ultima Teti, non laverebbe l'Oceano (carme LXXXVIII). Qui v'e, si, un commosso sentimento di sdegno, un concitato orrore di nefandezze: ma è notevole, per l'osservatore psicolugo, che il poeta ha aruto un vivace risveglio di onesta coscienza, sol quando si e visto tradito da Gellio: egli stesso eonfessa infatti in altro carme (carme XCI) esiergli stato congiunto di assidua eonsuetudine ed arerlo sperato fedele, non perchi il comoscesse probo o lo stimasse aborrente dalle infamie, ma perchi sapera che non "ra a fiellio madre o sorella culei per cui egli, il poeta, si strugrava di amore. Li cosi Catullo, se non interviene rivaliti $"$ golosia, fall (be indulga ogli stesso alle nefandezace cue ritrae, a almeno che vi passi in mezzo indif-

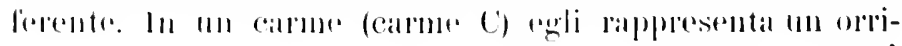


bile fraterno sodalizio di veronesi, del fiore anzi, come egli dice, dei giovani di Verona: Celio ed Aufileno, Quinzio ed Aufilena (1). Ed egli non ha un grido di orrore: ha una facezia: *Questo si che ì un sodalizio fratemo veramente dolce! » ed ha, incredibile a dirsi, un augurio a Celio, giacchè Celio gli si era serbato amico, quando una fiamma insana gli ardera le midolla: «Sii felice, o Celio, e possa in amore simugere al compimento dei roti tuoi!" Questo giovane ardente e passionato, avea bensì acquistato, per la fatale efficacia della corruzione profonda in cui vivera, una specie di msensibilità morale; ma nutriva intima e profonda la fedeltà all' amicizia e

(1) Nel v. 2 Flos reronensum . . iuvenum si riferisce grammaticalmente solo a Caelius e Quintius, ma non è dubbio che si riferisca per il pensiero anche ad Aufilenus ed Aufulenc. Questi nomi si trovano spesso nelle iscrizioni di Veroua e dintorni (seritti con doppio 1, come del resto nel cod. di Catullo Canoniciano 30 di Oxford a CX, 6 e CXI. 1). Aufilenà è la stessa che commettera incesto con lo zio (CXI, 4), e che frodò a Catullo il prezzo dei suoi favori, non dando poi ciò che avera promesso (CX). Quinzio è probabilmente il medesimo del carme LXXXII : uno dunque dei rivali fortunati con Lesbia. Ed altro rivale era Celio, eui si riferisce il carme LVIII, e eióe Celio Rufo, di cui discorriamo in ispeciale eapitolo. - Che poi il carme LXXXII a Quinzio, eosi caldo di passione fremente. si riferisca alla gelosia di Catullo per le relazioni di Quinzio con Aufilena, noi crediamo impossibile. Cosi interpreta quel carme lo Heskamp, De C. Valerii Catulli vita, p. 30. Quinzio amara Aufilena, eome risulta dal carme $\mathrm{C}$; ma quando insidiò a Catullo anche l'amore di Lesbia, Catullo ghli si rivolse eon le disperate parole del carme LXXXII, supplicando l' amico a lasciargli quel che gli era piì caro degli ocehi, se degli occhi r't qualehe cosa più cara. Più cara degli occhi era Lesbia; Aulilena non gli ispirò rhe sogghigni e scherzi atroci sulla sua perfirlia (carmi CX e CXI). 
la gratitudiue, sin quasi alla comnivenza, e almeno allindulgenza, per il delitto. Ma fu sua cupa idea che lungro la sua ria ei non trovasie quasi mai altro che traditori. Ed il poeta ha, per essi. ora parole accorate di rimprovero, ora parole violente di minacce e di rendetta; ed ora, cominciando in tono tenero ed affettuoso si esalta a poco a poco, e finisce con teribili imprecazioni. Così ad Alfeno (carme XXX) (1), egrli clomanda, com'è possibile che non abbia pietì di lui, del suo dolce amico, che lo abbandoni, che lo tradisca; e rammenta le antiche proteste di anicizia e fedelti, per conchiudere con l'auspicare la rendetta divina. A Gellio si rolge con tagliente ironia (c. XCl), rilerando che egri, il poeta, non credera che la lunga amicizia potesse essere causa sufficiente di tradimento, ma Ciellio, sì, il credera, perchè egli non sentiva gioia se non dore ela delitto.

Altrove gli si volge con tono sprezzante (c. CXVI): a lui basta coprirsi col mantello per evitare gli strali di Gellio; ma dagli strali suoi Gellio resterà confitto e così pagherà la pena. Ed anche in tono di baldanzosa stida si rolge a Ravido (carme XLI), che ruol rapirgli l'ogrgettudell'amor suo; ma il poetal gli ammunzia che to infitmerà in eterno (2).

(1) Chi i Alfono: Alfeno Varo: lo Schwaine (Gluacst. Calull.

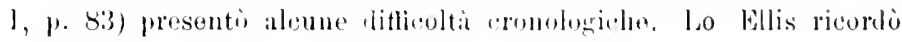
un I'. Alfeums, consul suffectus net $715 / 39$.

(2) Ravido it personaggio l'altrunle innoto. Nuterole in puesto

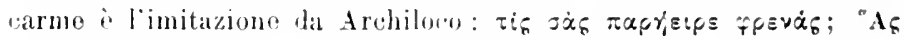

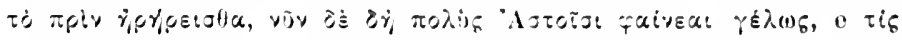

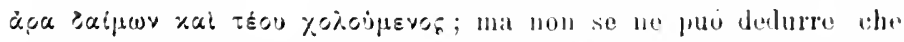
non si tratti di fatti e sentimenti rali, hensi di esoreitazioni pootiahe. Catullo aldatla ai casi smoi i motivi poetici di ani ba remi- 
Gemiti lisperati, che prorompono fuori da un'anima in tempesta, sono altri carmi di tal genere: quello a Rufo (c. LXXVJI), che gli ha straziato le viscere, e gli ha rapito ogni bene: quello dell' ignoto amico che dovera nutrirgli gratitudine ed invece gli si è fatto il peggior nemico (c. LXXIII) e quello all'altro, che nell'nltimo periglio lo abbandona, e non accoglie la voce di hui supplice (c. LX), od a Quinzio (LXXXIl), cui il poeta implora supplichevole, che non voglia togliergli ciò che gli è piu caro degli occhi, se degli occhi v'è qualche cosa più cara. Tutti traditori dunque questi amici, nei quali ebhe ad imbattersi l'infelice poeta? Gli è che egli, di estrema eccitabilità nervosa, immoderato nelle passioni, sentiva profondamente l'amicizia come l'amore; la sentiva in maniera che non poteva essere comune ai giovanotti del bel mondo romano. Traditori? Ma essi forse non si sognavano di tradirlo, e di fargli offesa, col godersi Lesbia, che si dava a tutti, e Catullo non lo ignorava, anzi era stato in principio acquiescente alle inferleltà di lei, per non diventarle noioso (c. LXVIII b, 95-100). Ma Catullo era giovane inesperto, che areva l'anima ardente di poesia, e vagante nei sogni; e col fatale inganno delle anime

niscenza. - Il Danysz. De srriptornm imprimis poeturnm romanormm studies atullanis (Posnaniae, 1876), p. 6:), ha: "Impudicitia ei ab Aurelio et Furio vitio data est (carm. 16), Ravidus 'puoque in eius carmina est invectus (carm. 40), quae opprobria facete et salse refutantur». $\dot{E}$ un curioso errore di interpretazione delle parole: Quaenam te mota mens... fyit praecipitem in meos iambos (v. 2). Il Danysz intende the Ravido sparlasse dei carmi di Catullu. Tutt'altro: Ravido roleva ruhargli Lesbia (r. $7-8$ : mens amores.... molnisti amared, e percio provocava i giambi di Catullo, ed andava a cozzarvi contro a precipizio (ayit praecipitem). 
semplici comincio and idealizzare la sla domna ed a volerla sllal, tutta sua, ed a macerarsi di sapere che tale essa non rra. e ad inveire contro chi si rantara della facile con'puista. Che piu: Pur quando Leshia is giunta all ultima abbirzime, e passa in fila $i$ duecento adialteri da trivio della sozza osteria, pure allora Catullo s'infuria e se la premle cun tutti i duecento, "li vuole tutti inmmare; - specialmonte prende li mira mo imnanzi a tutti, lgnazio di Celtiberia, dai lunghi capelli e dalla folta barba, e dal dente hianco Hibera defriontus mina (e. XXXVII) (1); sarri stato, tra i duccento, il preferito di Lesbia?: Probabilnonte i giovani mondani di Roma ridevano della cranle scalmana di Catullo, ni riconoscerano più l'antico (ompano delle arventure allegre. Catullo chiedeva forse "un po' troppo and essi, onl pretentere che considerassero come slla amante ma cosi vile entigiana. Eisli arera nei risuarli dellamicizia quei merlesimi trasporti di impetn e di artore. quella medesima sonsibiliti tenera e passionata, che poneva nellimore. Tra le anime indurite e ciniche dei griorinastri romani frest anima pia semplice conservava ancola nu poco dolla nativa sincerità e schietreza provinciale: e si manteneral firlo all’ amicizia pur nella colpa. e cominciara a rilualre la colpa e ad averne

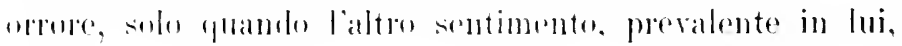

(1) Anche in XXXIX, in catullo memzinna fuesto stramo ben-

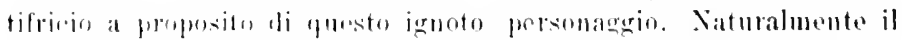

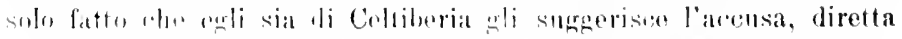

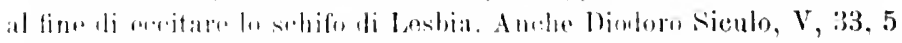

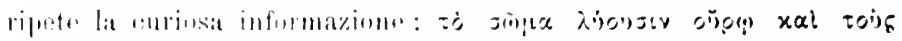

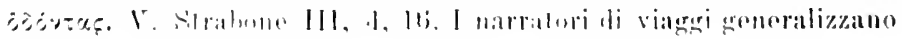
li solitn i rasi simpoli. in r.lli si sumb imbaltuli. 
ruello dell'amicizia, era, per il tradimento altrui, renuto meno.

$$
\text { *** }
$$

Un gruppo di poesie catulliane è dedicato ad una sua torbirla passione per l'amasio Giovenzio. I carmi or'è menzione di Giovenzio sono il XXIt, il XLVII, I'LXXXI, il XCIX: ma anche i carmi riguardanti Furio, e cioè XI, XVI, XXIII, XXYI (oltre il XXIV giá detto) debbono entrare in questa serie. Furio ì infatti uno degli amanti da Giovenzio preferito a Catullo: giacchè nel carme XXIV ¿̀ detto che l'amante preferito è uno quoi neque serros est neque arre (v. 5), e cii medesimo è detto appunto di Furio nel carme XXIII: "Furi, cui neque serros est neque arca $»$. Che poi ci debba entrare anche il carme XXV contro il cinedo Tallo ì opinione di moltissimi: si osserva infatti che Thallus i nome greeo che sembra poter corrispondere per il significato a Incentius. A noi cii non sembra sicuro; se Tallo ì Giovenzio, Catullo reme meno alla regola, che io credo costante negli elegiaci, che cioè il nome inrentato e poetico corrispondesse metricamente al nome reale.

Furio aveva per compangno di sue dissolutezze l'affatmatissimo Aurelio del carme XXI. Catullo infatti li nomina insieme. or trattandoli ironicamente (carme XI, ora insultandoli atrocemente come compagui di vizio (c. XVI).

Ora anche Aurelio i rappresentato come mo che aspirasse allamore di un fanciullo caro a Catullo, e gli stesse ai lati e tutto tentasse per riuscire (XXI). Non a probahile, come redremo, che si tratti anche nui di Giovenzio. e che quindi anche il carme $\mathrm{XV}$ e il XXI entrino in 
questa seric. Considerando che uno dei cami a Furio ed Aurelit. l' XI, ha una indicazione cronologica (r. 10 ('tersuris risens monimenta mayni ece.) si pui con bo Schwahe riferire tutta questa serie all'epoca clie succede alla rottura con Lesbia ed al viaggio di Bitinia, dopo il 699. V'e poi l'hospes pallido più di statua dorata, di Pesaro, a cui Giorenzio si is dato (carme LXXXI). Catullo si merariglia che excli non abbia trovato in tanto popolo un bellus homo da amare, ed abbia invece a curre e gli preponga quel messere. Si trattara dellostesso Furio: giacchè nel carme XXIV, che, come abbiamo detto a Furio si riferisce, Catullo fa che Giovenzio gli si rivolti e sli flomandi: "E che? Son è un bell'uomo? (v. $\mathbf{~}$ :

" (ui " non est homo bellus ? iniquies) (1).

Ciò che Catullo obbietta a Giovenzio e rinfaccia ai due rivali preferiti è che essi somo miserabili: Furio non ha serwo + non forziere (XXIII, 1: XXIY 5); la sua villetta e al riparo dai renti, ma is ciposta ad mu vento ben terribile e pestilenziale, nua ipoteca di quindicimila e duecento sesterzii (e. XXXVI).

Aurelio ir addirittura il padre di tutte le fami che fupollo. sono e saramo mai (e. XXL); e Catullo pensa che ora il suo fancinllo dora imparare al esurier a sitire (XXI, 10-11). Non puo quindi trattarsi di Giovenzio, giacchie questi nom cra sulle spese del smo amatore, anzi lo pasava, se a rio fa allusione il r. 1 del carme XXV:

(1) Giustamente lo Heskamp (lere C. Falerii Catulli rita oece, p. 33 asserva: a Hune furium non esse poetam Furium Biba-

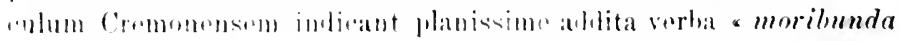

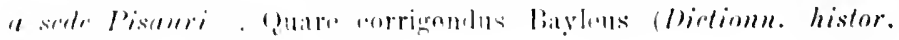
it rit., sulf r. C'ilull. s. 
Mallem divitias Midae dedisses Isti ecc. 》. Si tratta dunque di due amori puerili: Giovenzio. ritroso a Catullo (carme XClX: c. LXXXI, 5) cerde a furio (XXIV, 6): l'altro fanciullo par che a Catullo sia piu henevolo, giacchè non di sue ripulse si lagna Catullo, che anzi lo chiama meos amores (XY, 1: XXI, 4), meus puer (XXI, 11); bensì solo delle insidie che gli tende Aurelio per rapirglielo (XV, 9-13: XXI 5-î). Furio ed Aurelio erano una coppia di amici, che già arevano aruto relazione con Catullo fin dagl' inizi della sua passione amorosa, e il canzonarnno quando lessero la poesia sui baci, il carme VII ("Quaeris quot miki basiationes): se si sfoga a dar baci, essi motteggiarono, vuol dire che non è huono ad altro (XVI, 12-13). E Catulto rispose ad essi ferocemente (XVI. 1 e 1t: "Pedicato ego ros et inrmmabo " P'oi renne la rottura con Lesbia. Il carme XI ci fa quasi supporre che i due socii si fossero offerti pacieri, ed aressero fatto a Catullo protesta di amicizia e di ferelti a tutta prova. Catullo rammenta ironicamente cotali proteste (XI, 1-13), respinge i due, e manda per mezzo di essi l'espressione del sno disprezzo a Lesbia (XI, 15-20).

E riene ora il periodo degli amori puerili. I due sozii non erano tra di loro rivali: hensi entrambi erano rivali a Catullo: questi era respinto da Giovenzio, amasio di Furio, ma tenera un altro puer, che Aurelio tentava insidiarsis: indi l'irrompere delle contumelie e delle minace di Catullo contro Aurelio (XV. XXI) e contro Furio (XXIII, XXIV, XXVI, LXXXI).

Una curiositi di questa serie di carmi è la ripetizione quasi letterale del medesimo girn di parole. ma volta per Aurelio ed un'altra volta per Giovenzio e Furio. Si regga: 
XXI, 1-3: Aureli pater esuritionum.

Non harum modo, sed guot ant fuerunt

Aut sunt aut aliis erunt in annis...

XXIT, 1-3: 0 qui flosculas es Juventiorum

Non horum modo. sed quot aut fuerunt.

Aut posthac aliis erunt in annis.

l'er quanto riguarda Giorenzio, non i il caso naturalmente di disquisizioni storiche su questo putido personalfrio: solo usserviamo che come fu wia osscrvato dallo. Selowahe ( dal Benoist, non dovera essere straondinario il caso di wioranetti appartenenti a nobili famiglie. che gimngessero a talo estremo di ahbiezione, se Cicerone potì scrivere ( $1 / \mathrm{l}$ Att. I, 16, 25): " etiam noctes mulierum atque adulescentulorum nobilimm introductiones nommllis iudicibus pro mercedis cumulo fuerunt $»$. Lal famiglia dej Giovenzii era di Tuseolo (Cic. P'o Plome. V'IlI, 19 e XXIV, 58).

Quanto abbiamo deto basta, pillmi, a mostrare quanto poro sia comsistente l'opinione dello Harnocker (I)as Patullus Inrentiuslieder in Sene fubll. fiie lilass. Philol.

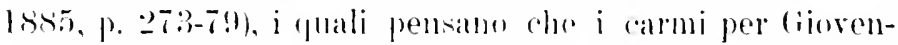
zin sieno scherzi pretici di imitazone alessandrina, e che Catullo nom abhia in essi rappresentato ma slat passione rale. Bene usservo il Ciandightio Cantores Euphorionis, Bologna, 190t, p. 101) dhe l rersi i-t del carme LXXXI ed i particolari risuardanti furio od Aurelio non sembrat116 darroro riferirsi a cireostanze immaginaries: e noi an-

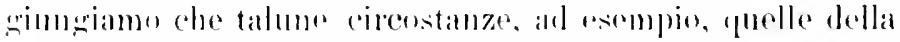

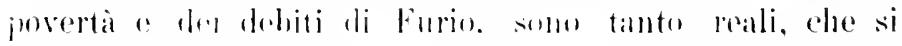
trowano ancele in almi (XXll, 1 ( XXYl) ove, non

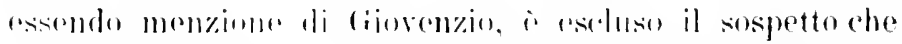

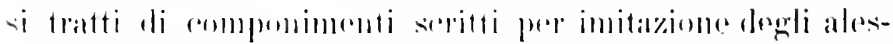

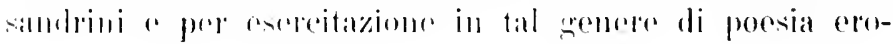


tica. In verità non intende l'arte catulliana chi non vi ravrisa sempre il fondo realistico e puo pur solo supporre che possa trattarsi di esercitazioni poetiche sopra circostanze fittizie.

$$
\text { *** }
$$

Sette epigrammi di Catullo (1) sonn diretti contro an terribile rivale. Gellio. $\mathrm{E}^{\prime}$ un hen tristo persmatgrio. Catullo ce lo dipinge coi colori piu neri. Cili era stato amico, ed anzi a lui congiunto di intima consueturline: ce lo dice il poeta stesso (XC. 1, 7): " Et quamris tecum multo coniungerer usu». E lo arera sperato fido, ed areva creduto che la intimità non fosse ragione sufficiente per il tradimento (ivi, v. 1 e 8). Per cercare di abbonirlo con qualche manifestazione affettuosa, affinchè Gellio non gli scagliasse le sue perfide saette, era andato per lui cercando i carmi di Callimaco per mandarglieli tradotts (CXVI, 1-4). Ma fu vano il suo laroro. Catullo gli minaccio la sua terribile rendetta! (CXVI. 5-8). E tenne la parola. Gli epigrammi sono tutti sopra gli orribili vizii di Gellio. L'incesto con la mai!re ri occupa gran parte; e l'epigramma XC è ad esso de!licato: ma non è tutto: l'epigramma LXXXVIII ci rappresenta (iellio che, discinta la tunica. insomne per il prurito della madre e della sorella e della moglie dello zio; e più ancora agcriunge l'epigramma seguente: Gellio è cosi magro, perche si consuma con madre, sorella, zio, con tutte le parenti di casa: egli non tocca se non ciò che è delittn il toccare. Ed ancoral In

(1) Cami LXXI, LXXX, LXXXYll, LXXXI, Xe. Xo. CXVI. 
zin non volera scamlali: ed eceo che fiellio per accontentarlo gli sramola la moglie, e cusi ora, anche se vuole imrmmare lo stesso zin. questi deve starsene tacito (LXXIV). Ma ciellin stesso i mn immmatus; e di tal suo vizio orrendu daran prova i rotti fianchi del misero Vittore o le sur lahhra tinte di munto siero (LXXX).

Ma chi is dunque questo terribile Gellio? Fu pensato per lungo tempo a L. Gellio Publicola, del partito di Clodio. contro il quale con prande veemenza si scaglia Cicerone nella orazione Pro Sestro (cap. 51 e 52). rapprentandolo come nomo dedito ale dissipazioni an ai rizii, che areva sposato nua libertina, mon libidinis cansa, dite ironicamente Cicerone, sed "t plebicoln videretur, accennando anche al orrendi vizii con le parole piene di significati sottintesi: "est me ultus, rmm illo ore inimicosest meos saciatus", e chimandolo in fine furiosissimms atque egentissimms ganeon, al altrove (In Vat. 2, 4) mutricoln seditiosorum ommimm.

Son peri a questo diellio sono diretti nali epigrammi di Catullo. (questi era il profimus menzionato da Catullo; grli epigrammi son diretti al degmo nepote (1). Già redrommo come (iollin ela stato companno di piaceri e di arventure a Catullo: bisognerai dungue pensare ad un coetano di Catullo: colel resto il poeta accenna ad meta molto grovanilo di ciollio dicomdo (l.XXX, 1) " rosea

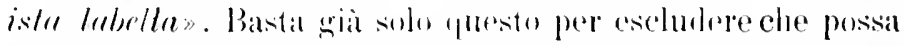
trattarsi di quel follio Clondiano, eontro il quale inveisce

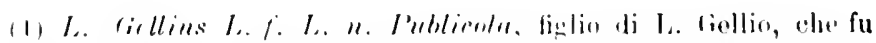

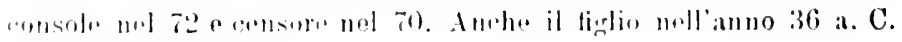
oflenne il consolate. 
Cicerone, giacchè quello dovera esser vicino alla cinquantina quando Clodia areva rentinove anni (1).

Vediamo dunque del nepote. Il padre suo, L. Gellio, che fu console e censore, ripudio la moglie Pola, e trasse in isposa un'altra donna, che el'a dunque matrigna all'adolescente Gellio. Ma a proposito di ciò Valerio Massino riferisce una notizia, che vale molto ad illustrare storicamente i carmi di Catullo.

Dice dunque, di L. Ciellio padre, che il figlio fu accusato di terribili delitti : l'incesto con la matrigna ed ll tentativo di uccidere il padre. L. Gellio non trascorse immediatamente alla vendetta, ma convocò i senatori ed invitò dinanzi al essi il figlio alla discolpa; ed esaminata con essi la causa lo assolvette (2).

Sarà stato imnocente lavrero? In tali casi l'assoluzione è un atto politien, compiuto per evitare guai maggiori e non gettare ombre sul nome familiare. Ad ogni morlo all' innocenza non sarebbe stato punto disposto a credere Catullo.

Il quale anzi esagerò le cose, e non parlò di noverca, ma rli mater, e ri aggiunse tutto quell' altro po' po' di roba. Certamente non le invento lui quelle accuse: le rac-

(1) Cfr. Schwabe, Quest. Catull. p. 101 sgg. Il Westpbal, Catulls Gedichte.... Breslat, 1867, suppose che i carmi LXXXVIII, LXXXIX, XC, XCl si riferissero all'adolescente, $\theta$ gli altri LXXIV, LXXX, CXVl allo zio. Sarebbe molto strano che Catullo avesse ingenerato tal confusione, iudicando indifferentemente con un solo nome l'nno e l'altro. Del resto i sette epigrammi sono cosi connessi, che è vano il tentativo di disgiungerli. Con prolissità e sotigliezza soverchia confuto il Westphal il Rettig, Catnlliana III (1)e epigrammatis in Gellium striptis). Bernae, 1871.

(2) Val. Mass, V, 9, 1 . 
colse lalla società in cui virera. E per quanto si rogliano fare riserve e limitazioni, qualche cosit di vero ci dovera essere: e, ad esempio, nulla di strano v’ò che l'adolescente fiellio avesse anche relazioni con la moglie dello zio, graccho Cicerone sembra volere accennare alla disonestì di lei, quando mette in rilievo che essa era una libertina, e che Gellio, il putrums, l'avera sposata libidiuis cullsa (1).

$$
\text { * } *
$$

Sullo sfondo di questa sucietà torbila e triste si anita e grandeggia nella vita di Catullo, la figura di una donna. E quella donna, benchi indegnissima dell' amor suo, gl'ispirò una passione. che fu il tormento della sua vita mortale, ma gli dischiuse le vie gloriose della mande arte. Altri, nelleti sucessiva, potè adoperare più squisiti artificii e più fini eleganze, ma questo giorane e sciaguratu veronese ebbe roci che passarono i secoli, per larlore e la sincerita del sentimento, che le fece sciturire dalle intime fibre della sua anima dolorante. Egrli portio nella sua poesia l'ispirazione dell' amore rero, con le sue esultanze trionfali, con $\mathrm{i}$ suoi abbattimenti, con la rahbia delle sue gelosie, con i suod gemiti disperati di angoscia. Eoli sispiri al suo sentimento a di esso fece, con profondo e spietato studio, l'analisi. I modelli che grli stavano dinanzi cantavano per la magregior pante l'amoro in Inta maniora ancor treppo superficiale, risentivan an-

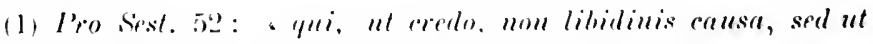

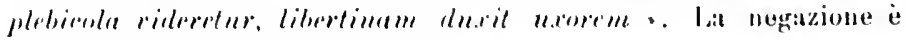

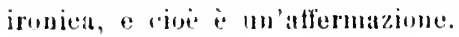


cor troppo dellobhiettivitì di tutta l'arte greca. Catullo penetra addentro nelle passioni del cuore umano e le esamina

Egli fa, per esempio, lanalisi della sua passione amorosa: egli sente che la rita da lui vissuta, lungi dalle cose del mondo, e dedicata tutta ai piaceri, e all amore, questa vita che egli chiama con mua sola parola otimm, è la sua rovina, come fu già la rovina di monarchi e d'imperi (LI, 10):

Otium. Catulle, tibi molestum est:

Otio exultas, nimiumque gestis;

otium et reges prius, et beatas

Perdidit urbes.

È nei suoi gioni felici: Lesbia gli giura amore eterno, immutabile, ma egli non si lascia acciecare dalla passione, sino al punto di non dubitare della sinceriti di quelle promesse (c. CIX, 3):

Dii magni, facite, ut rere promittere possit

Atque id sincere dicat et ex animo.

E nei giomi del contrasto: Leshia va sparlando sempre di lui: dunque, egli dice, m’ama! Qual'è la prora? Anche egrli la maledice continuamente ed anche egli sente di amarla (XCII):

Lesbia mi dicit semper male, nee tacet umruam

De me: Lesijia ine, dispeream, nisi amat.

Quo signo: "luia sunt totidem nea: deprecor illam

Assidue: rerum disueream. nisi amo.

E lo stesso pensiero che ricorre in un altro carme ancor piir caratteristico (LXXXIII): Lesbia innanzi a slo marito sparla di Catullo, e quegli ne gongola dalla gioia, e 
non comprende, che se Lesbia inmemore di Catullo non ne parlasse, sarebbe guarita dalla passione amorosa, ma giacchè questo non is non solo se ne ricorda, ma ne è silegnata: cioe arde d'amore.

11 poeta non si dissimula la grarità della condizione che egli si i imposta; legare la propria vita al capriccio di una donna ineostante, le cui promesse in rento et rapilla scribere oportet arun (LXX, 4): egli sente che ormai non potrà mai più stimare quella donua, nè potrà mai cessare di amarla (LXXV, 3-4):

Lt iam nec bene velle queat tibi. si optuma fias,

Nec desistere amare, umniat si facias;

ed i qui tutto lo strazio dell animo suo, tutto il contrasto tra l'odio e l'amore, ele gli tumultuano nell' animo. e che egli esprime nel breve epiglamma (LXXXV):

(1) et ano. (yuare id faciam, fortanse requiris:

Nescio: sed fieri sentio et excrucior.

Si regrag dunque qual profonda analisi della slla passime amorosa ci fresenta Catullo, e come tutti egli ritragga gli slanci dellardore, e sli sconforti del dubbio. l: percio noi assistiamo impressionati al tramma che ci $\therefore$ srolge dinanzi nei suoi (armi, percio noi rimpiangiamo quella giovine esistenza, quella nohile e passionata natura, che peri sul fiore degrli ammi, spirando ylasi come in un alite caldo di volutti e d'amore. Satura nohile e passionata, abhiam detto; ad infatti si troverobbe dittioilmente a chi paragonarlo per l' imitahiliti sotremat dei sentimenti suri, motat con i tratti gentili, ehe hrillanu nel suo earme

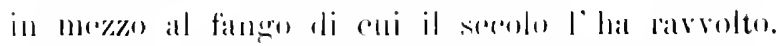

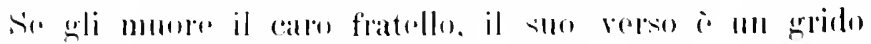
straziante di dolore (1.XVlll a, 20): 
0 misero frater adempte mihi.

Tu mea, tu moriens fregisti commoda, frater, Tecum una tota est nostra sepulta domus!

Se Quinzio tenta rapirgli il cuore della sua donna, col pianto nella voce egli lo supplica di non rolergli rapire ciò che gli è più caro degli occhi, se vi ha qualcosa più cara (LXXXII); se Lesbia dopo un contrasto gli si offre spontaneamente di nuovo, chi potrà più rattenere la sua gioia che irrompe (CVI, 7):

Quis me uno vivit felicior? aut magis hac res Optandas vita dicere quis poterit?

Se poi, disingannato ormai dall'amore di Lesbia, cerca di affogare i molesti ed incessanti ricordi nelle ebbrezze della voluttà, con quale sprezzo parla di queste donne che si prestano ai suoi piaceri, di Ametina, di Aufilena, ed anche di quell' Ipsitilla, che egli chiama meae deliciae, mei lepores (XXXII).

Infine quando quella Lesbia è giunta al colmo della prostituzione, con quale sprezzo pieno di rammarico, pieno ancor quasi di fremiti, lo annunzia rogli all' amico Celio (LVIII):

Caeli, Lesbia nostra, Lesbia illa. Illa Lesbia, quam Catullus unam

Plus quam se atque suos amavit omnes.

Nunc in quadriviis et angiportis

Glubit magnanimos Remi nepotes.

$$
* *
$$

Ma chi fu dunque mai questa Lesbia, alla cui perfidia, dobbiamo le più commosse roci di dolorosa passione, che 
risuonassero nella lingua del Lazio? Che si tratti di falso nome, non v'e luogo a dubitare, per la testimonianza di Ovidio (Trist. II, 428), che così dice di Lesbia:

Sic sua lascivo cantata est saepe Catullo

Femina, cui falsum Lesbia nomen erat.

Ed una notizia pii determinata ci fu data da Apuleio. La Lesbia di Catullo si chiamava Clodia, come si chiamava Metella la Perilla di Ticida, ed IIostia la Cynthia di Properzio, e Plania la Delia di Tibullo (1). Fin qui Apuleio: e possiamo aggiungere che a questo melesimo ciclo di denominazioni poetiche appartengono la Lencadia di Varrone Atacino, la Lyeoris di Comelio Gallo, la Corinna di Ovidio. Questi poeti romani, $m$ po per seguire l'uso alessandrino, un po' per naturali riguardi umani, e sopratutto per evoeare immagini grentili di miti o di poesia, cercavano nomi greci alle loro amanti: ed era naturalmente loro vanto rendere quei nuovi nomi poetici più famosi, che non fossero, per antiche virtù e magistrature, famosi i nomi veri delle famiglie romane, cui esse apparteneva$n$ (2). Cuencadia fu forse chiamata da Varrone Atacino

(1) Apuleio, Apol. 10: allic illud reprehendi animadvertisti, quod cum aliis nominibus pueri vocentur, ego eos Charinum et Critiam appellitarim. Eadem opera accusent C. Catullum quod Lesbiam pro Clodia nominaverit, et Ticilam similiter, quod quae $\mathbf{M e -}$ tella erat, Perillam scripserit, et Propertium qui Cynthiam dicat, IIostiam dissimulet, et Tibullum yuol ei sit Plania in animo, Delia in versu ". Che nell'uso di questi preti il nome fittizio dovesse corrispondere metricamente, per numero o quantita di sillabe, al nome vero, in modo the fosso sempre possibile sostituire quest'ultimo, fu notato primamente dal Bentley (Ad Horat. Od. II, 12, 13).

(2) Cfr. Properzio I, 11, 9-8; 1, 15, 25; II, 24, 1; Ps. Verg. ratat. 1X, 23 sagg.: a Felirem ante alias o te scriptore puellam, Altera non fama direrit isse prior. 
l'amante sua, per ricordo della rupe di Lencade, famosa per la leggenda di Saffo: i nomi Dèlia, Cinzia e Licori furono dati per riguardo ai soprannomi di Apollo, protettore delle Nuse; Corinna ricorda l'antica poetessa e Lesbia ricorda la gloria di saffo. Null'altro dagli antichi sappiamo sopra Lesbia: Properzio rammenta che essa per il verso di Catullo divenne più famosa di Elena (1); nulla d’importante ci apprendono le menzioni che se ne hanno in Marziale e Giovenale (2); Sidonio Apollinare le fa commettere, oltrechè peccati d'amore anche peccati di poesia (3). Non sappiamo onde egli prendesse la notizia, la quale nou ha però nulla di strano: queste domne eleganti della corrotta società romana prenderano qualche volta troppo sul serio la loro parte di Muse ispiratrici dei poeti; e basti per tutti l'esempio di Cinzia verseggiatrice! (cfr. Prop. I, 2, $2 \vec{\imath}-30$ ).

$$
* *
$$

Ma noi sappiamo invece non poco intorno alla Clodia, con cui la Lesbia è da identificare: ^ cla quel che sappiamo la identificazione è pienamente confermata (4). Clo-

(1) Properzio, II, 34, 87 [=III, 32, 87 Muell.]:

Haec quoque lascivi cantarunt scripta Catulli,

Lesbia quis ipsa notior est Helena.

V. anche II, 32, 45 [= III, 30, 45 Muell.].

(2) Marziale VII, 14 ; VIII, 73; XII, 44, sgg.; XIV, 77; (iivvenale, Sat. VI, $7 \mathrm{sgg}$.

(3) Sidonio II, 10.

(4) Quanto sieno di suarso valore le obbiezioni sollevate più volte contro l'identificazione di Lesbia con Clodia, si può vedere 
lia, della nobile famiglia dei Claudii (1) fu figlia di Appio Claudio Pulcro, che fu pretore nel 665 di R. e console

nel volume di A. Couat, Étude sur Catulle, p. 271-272. Da questa jagina riportiamo la prarte che riguarda le meno insulse di tali obbiezioni: * Lesbie, si elle eut éte la mème femme que Clodia, aurait été plus igée que Catulle. Il n'y a là rien d'invraisemblable: cette différence d'àge expliquerait plutit la ténacité de la passion de Catulle, et si dans une le ses pièces il lui déelare qu'il l'a aimée comme un père, il n'y faut point voir une contradiction, mais seulement une expression naturelle dans la bouche d'un homme s'adressant ì sat maitresice. Enfin. comment Catulle, paurre poète, homme après tout assez ofscure, se serait-il vanté d'un adultère avec une patricienne: Il n'avait fait mobablement que succéder ì d'autres, comme d'autres lui succimlirent, et les intidélilés de Clodia, mème aver des gens le petite condition, étaient célèbres ».

Dall'apascolo del Fennor Cuationes Catullianae (Barmen, D. B. Wiemann, 1896. 1. 24) traguhiamo l' enumerazione, the egli fa, delle ragioni che portano a confermare la notizia li Apuleio eirea l'identità di Lesblia con Cloulia: «1. Et I.eshia et Clodia quadrantaria nobili lono ortae eraut. ¿. Lesbiam nuptam fuisse scimus, Clodia erat uxor Q. Mtelli Celeris. 3. Ltrapue femina decepit maritum stumarge fecit multis cum abulescentibus. 4. Et Clodiae et Leshiae maritus indole et ingenin videtur caruisse. 5. Utraque femina pulcherrima fuit. 6. Ttrajue mulier ingenio insignis fuit. 7. Clodia a M. Caelio Rufto oratore amata est, Catulli Lesbia eodem tempore cum Caclio linfo quonlan rem hahuit. S. Clodiam eum P. Clodin fratre incestum fecisse scimus. Catullus a Lesbia Lesbium sibi anteponi dicit. Si lesliace verum nomen est Cloelia, illius hominis nomen Clorlins lumit necesne est. Lesbius qui a Lesbia poetae antefonitur. fuit l'. Clouline l'ulelere, 'fuadrantariae frater. 9. In

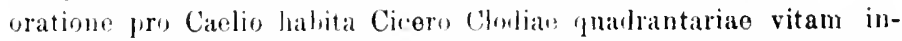
quinatiscimam describit. Quae imang plane congruit eum imagine Leshiae magis magistue depratratace at Catullo exhibita $\gg$.

(1) Sullatermarsi delle forme clandius e clodius rimandiame, a

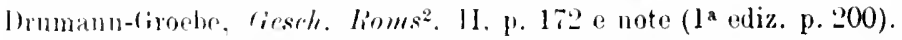
II lindsay, Latin langurger, p. 41, pone clodius come la forma 10pwlare, mottata la rhi voleva ingraziarsi il favore dol popolo. 
dieci anni dopo. Essa fu moglie di Quinto Metello Celere, infelicissimo nomo, morto improrrisamente nel $694 \mathrm{di}$ Roma, ed in modo cosi misterioso ed inopinato, che da tale morte non fu alieno il sospetto di coniugale insidia (1).

Fu rotta a tal dissolutezza di costumi, che le reme dato il nome di Clitennestra quadrantaria, e tal nome che designara oltrechè l'efferatezza del delitto compiuto, anche l'arrilimento della prostituzione piu abbietta (2), essa si sentì rinfacciare dalla reemente parola di Celio Rufo e di Cicerone (3). Ebbe per fratello minore P. Clodio Pulcro, il famoso nemico di Cicerone. La roce comune fu che tra il fratello e la sorella intercedessero pratiche incestuose. Di che avremo ancora occasione di toccar bre-

(1) L'accusa è in Cicerone, Pro Caelio, 24,59: "quem rirum si nulla ris repentini sceleris sustulisset, quonam modo ille furenti fratri suo consulari restitisset *. Anche Celio Rufo nell'orazione in propria difesa, chiamò Clodia Clitemnestra quadrantaria (cfr. Quintiliano VIII, 6, 53). L'attendibilità dell' aceusa fu messa in dubbio da qualche moderno (ad es. Schmidt, nei Prolegomena della sua ediz. p. XVIII sg.). Ai dissidii di Clodia col marito accenna Cicerone in una lettera ad Attico, II, 1, 5: * Ego illum odi male consularem. Ea enim est seditiosa, ea eum viro bellum gerit, neque solum cum Metello, sed etiam cum Fabio, quod eos mihi esse amicos moleste fert 》.

(2) Quadrantaria significa che per un quadraute si prostituiva.

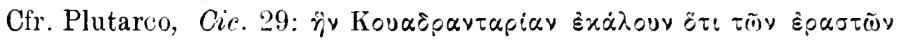

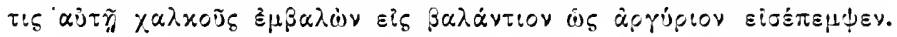
Altrimenti il Baehrens, Comment. p. 34: * Lacabantur Romae quadrante riri (Inven. I'I, 147; Sen. epist. 86, 9), videturque Clodia aliquando una cum riris lavasse: quae res sub Caesarum imperio non ita rera tum noritate sua ita offendere potuit, ut hinc feminue mocaci lescivaeque eognomen illud inditnm esse non sit veri dissimile.

(3) V. in questa pagina la nota 1. 
remente tra poco, a proposito di un epigramma catulliano.

Di questa perdutissima domna fece una efficace dipintura Cicerone nella orazione famosa in difesa di Celio Rufo. Era questi giorane ardente ed impetuoso, dedito alle lettere ed anche alla vita galante, che si era gettato nel turbine politico con tutta la foga della sua giovinezza. Avera preso di mira importanti personaggi, promovendo contro di essi gravi accuse ed ottenendo talvolta vittoria nell arringo giudiziario. Tanto più vigoroso fu dunque lo sforzo dei suoi nemici per perderlo. Gli intentarono un processo presso il pretore Cn. Domizio Calvino, lanciandogli gravissime accuse: che egli avesse a Napoli eccitato sedizioni contro la repubblica, che egli aresse dilapidato beni altrui, battuto a l'ozzuoli i legati Alessandrini, preso a prestito denaro da Clodia, per corrompere i servi li L. Lucceio ed indurli ad assassinare Dione, capo della logizione alessandrina, infine che egli aresse tentato di togliere di mezzo col veleno la stessa Clodia (cfr. Pro raclio, $51 \mathrm{seg}$.).

Era dumpue Clodia uno dei perni dell'accusa. Intorno a lei si erano stretti i nemici di Celio, ed ella dava loro testimonianze e prove per vendicarsi dellantico amante, che l'aveva oral abbandonata, e per isfogare contro di lui la sua rabbia grelosa (1). Cicerone non fu il solo di-

(1) Cfr. l'urazione Pro Caclio, s 75, nel quale si loda Celio, per essersi liberato dalle spire di Cludia. Circa l'ira di Clodia cfr. 55 inminiea domus.... Irata mulier; 61 discidium extitcrat. Celio si era recato ad abitare nella casa di l'. Clodio sul Palatino, per essere vicino alla surella di lui, e prese parte alle orgie di Clodia a lioma enl at Bata. ('fr. I'ro Caelio 18, 35-38,74, 49, 61-63, 75. Cio avernue nel 6965/5s; cfr. Sichwahe, (Marst. Catull. p. 64 sgg. 
fensore di Celio. Egli stesso, l'accusato, parlò in sua difesa (1), e dopo di lui II. Crasso (2), e poi Cicerone, che riassunse tutta la causa e contribuì certo non poco all'assoluzione del suo difeso. Ma nell' orazione di Cicerone tiene il campo la figura di Clodia, malvagia, corrotta e corruttrice (3). Lioratore assicura solo tanto volere trattare di Clodia, quanto si attenga al suo compito di difesa (\$ 31); ma non risparmia occasioni per lanciare frecciate e punte ironiche ed aperte ignominie contro la terribile donna.

Se gli capita di menzionare il fratello, egli lo chiama senz' altro marito, riprendendosi poi subito dopo: "volevo dire fratello, ma qui sbaglio sempre » (s.32): se dichiara di non volere contro sè eccitare le inimicizie della donna, scherza sulle sue molteplici amicizie: " tutti l'ban creduta sempre piuttosto amica di tutti che nemica di qualcuno» (ivi).

E con pochi efficaci tocchi descrive la dissolutissima vita di lei, che a Baia non cercava neppure di nascondere nelle tenebre e nella solitudine gli eccessi della sua libidine, ma anzi apertamente ne godera, in mezzo alla gente ed alla luce del sole $(\$ 47)$. "Se una donna maritata, egli dice (\$ 49), aprirà la sua casa alla lussuria di tutti, e spontaneamente e apertamente intraprenderà

Sopra i carmi di Catullo contro il rivale Rufo, o diretti amichevolmente a Celio, r. sopra, cap. IV (carmi LXIX, LXXVII, LVIII, C)

(J) Cfr. Pro Caelio 45; Quintil. VIII, 6, 53; XI, 1, 51; Suetonio, de rhet. 2 ; Festo s. v. Oreae.

(2) Cfr. Pro Caelio 18 e 23.

(3) Le notizie tramandate dagli antichi sopra Clodia veli in Drumann-Groebe, Geschichte Roms, II, (Leipzig, Borntraeger, 1902), p. 314-319 (Drumann, 1a ediz., p. 376-381). 
vita meretricia. e frequenterì $\mathrm{i}$ banchetti di nomini estranei, e se questo ella farà non in Roma soltanto, ma negli orti e in mezzo alla folla di Baia; se infine non nell iucedere soltanto, ma altresi nexli ornamenti e nelle compagnie; non solo per l'ardore degli occhi o per la licenza del dire, ma altresi per gli abbracciamenti, per i baci, per i bagni, per le passeggiate in barca, per i conviti, si diporterà in tal modo da sembrare non solo meretrice, ma proterva e sfacciata meretrice, un giorane che per caso le si unisea, ti parrà, o L. Erennio, un arlultero o un amante? uno che voglia espugnare la pudicizia, o soddisfare le sue voglie?» La risposta non poteva essere dubbia per Celio, che era viovane di mondo: ma il povero inesperto Catullo rimase irretito, e continuó ar amare la sciagurata, pur quando la vide al fondo di ogni deprarazione (1), circondata dai suoi cento e duecento adulteri della sozza osteria (르). Se questa i la rappresentazione che Cicerone fa della vita di Clodia, in mezzo alle delizie e al lusso dei luoghi di bagni e di piaceri. nou manca nelle sue pagine la rappresentazione della vita che essa traera a Roma, circonlata da servi. che erano amanti, e che usavano con la pardrona ogni licenza e familiarita, in ma casa nella quale crano comuni le dissolutezze, le libidini, la lussuria, tutti i vizi e le vergogne più inaudite (\$ 5 T).

E Cicerone descrive anche i frequentatori di quella casa: Sicno pur ruanto rogliono nej conviti, faceti, spiritosi, talvolta anche perche eccitati dal vino, facondi: altro è valere nel foro. altro nel triclinio; altra cosa sono gli sgabclli ed altro i letti: mon hanno la stessa faccia i giu.

(1) Cfr. i armi Xloll, J,VIII IXXV.

(2) Cfr. il carme $\times \times \times$ VII. 
dici e i commensali: la luce del sole è molto diversa da quella dei candelabri.

Per conseguenza, se costoro compariranno, prescindiamo da ogni loro delicatezza e facezia. Ma stiano a sentire a me, pensino ad altro, si procurino altro farore, si dimostrino in altre prove, valgano presso cotesta femmina per bellezza, la dominino col lusso, le stiano accanto, con lei si giacciano, a lei siano schiavi: ma risparmino il capo e la fortuna di un innocente » (\$ 67 ). Il pensiero ricorre spontaneamente a Catullo: non sarà stato talvoltal tra i lauti iuvenes dei quali parla Cicerone?

Nè meno evidenti sono gli accemni ciceroniani agli orrori dell' incesto fraterno (1), quando rappresenta il minore fratello, che, per non si sa quale timidezza, e per notturni vani timori, ha voluto sempre dormire con la maggiore sorella (\$36), e quando in fine (\$ 78 ) esorta i giudici a non abbandonare M. Celio alla libidine della muliebre vendetta, affinchè non si dica che una sola femmina col suo consorte e fratello abbia potuto e salvare un turpissimo assassino e rovinare un onestissimo giovane $(\$ 47)$.

Tale era la Palatina Medea (\$18), la mulier potens quadrantaria (\$ 62), la femmina che congiungeva ogni giorno nuovi turpissimi amori, amorum turpissimorum

(1) Nell'orazione Pro domo, 34, \$. 92 così dice con atroce celia Cicerone: "Non tam insolens sum. quod Iorem esse me dico. quam ineruditus quod Hinerram sororem Iovis esse existimo. scd tamen ego mihi sororem virgincm adscisco: tu sororcm tuam virginem esse non sisti: sed vide ne tu te debeas Iovem dicere, quod tu iure eundem sororem et uxorem appellare possis». Questo paragone con Giove fa pensare al giuramento che Lesbia faceva ripetutamente a Catullo, che essa preferiva cioè Catullo allo stesso Giove (LXII, LXX). Che Cicerone conoscesse da indiscrezioni il motto di Lesbia, e ne celiasse? 
foedera (\$ 34), la femmina alla quale parera convenirsi tutto ciò che si potesse dire di turpe (\$ 69).

Tale la femmina, per cui si macerò Catullo in fremiti di passione e di gelosia. Niuna forse delle turpitudini di Clodia gli fu col tempo ignota. All̈incesto col fratello egli fid allusione nel carme LXXIX:

Leshius est pulcher: quilui? quem Lesbia malit, Quam te cum tota gente, Catulle, tua?

Sed tamen hie pulcher vendat cum gente Catullum, Si tria notorum savia reppererit.

Se Clodia ¿̀ Lesbia, i naturale che Clodio sia chiamato Lesbins, Ma Lesbius est pulcher: si tratta, oltrechè del hello aspetto di Lesbius, anche di un ginoco di parole: riacchò il nome intero era $P$. Clorlius Pulcher. Anche Cicerone scherza sul cognome, chiamando Clodio pulcelliss puer (Ad Att. I, 16, 10) (1). La punta dell'epigramma Catulliano pare stia in cii: che Lesbio è bensì un hel griovane, per molo che Lesbia lo preferisce a Catullo, ma ò così ore inquinatus che Catullo ne rifiuta i baci, ed egrli si consolerebbe del rifiuto di Catullo e venderehhe Catullo con tutti $i$ suoi, se potesse trovare tre baci di persome conosciute, e cior dei sriovani obsoleti (che lo fungrono per il siro fetido fiato).

$$
* *
$$

Catullo giunse naturalmente a poeo a poeo. gradualmente, alla comoseonzal di tutta l'atroce rerita, beve a

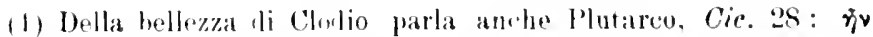

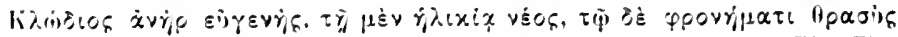

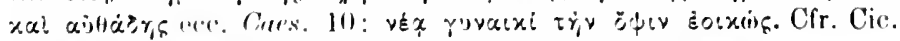
In Clod. et Ciur. 5, 30.1 ; In harusp. resp. "21, 44. 
sorsi il calice amarissimo. Rifare la storia dell'amor suo dalle sue poesie non è possibile nei minori particolari: ma a grandi tratti le fasi di questa passione si scorgono. E questa storia rassomiglia a quella di tutte le altre grandi passioni, con le loro alternanze di gioie e di amarezze, di accordi e di dissidi, col succedersi dei dubbii angosciosi, degl' impeti frementi, degli abbandoni roluttuosi, delle gelosie, dei tormenti, delle disperazioni supreme.

Il poeta, come sogliono fare i poeti, aveva cominciato ad idealizzare la sua donna: la sua fervida dichiarazione di amore è una poesia tradotta da Saffo (LI): il poeta dice pari ad un Dio, anzi superiore ad un Dio, chi assidendosi di fronte a Lesbia, può guardarla ed udirla rolce ridente. Erl i suoi carmi in questa prima epoca di felicità sono tutti rappresentazioni di estatici rapimenti: esortazioni a vivere ed a godere, in obliosa spensieratezza (V), molli desiderii di baci innumerevoli (VII). Il poeta stesso rammenta quest'epoca della sua prima felicità, quando nell’ ospitale casa dell’ amico Allio gli si presentava la sua candida dea dal molle piede, sostando sulla soglia ed irraggiando intorno fulgore (LXVIII b, 27-32).

Ma cominciarono ben presto le prime infedeltà. Al poeta parve in principio gesto spiritoso rassegnarrisi per non diventare molesto alla sua signora, e si consolò con l'esempio di Giunone, la massima delle dee, che pur ebbe a sopportare i molti furti amorosi di Giore (LVIII b, 95140). Non molto a lungo però fu così paziente Catullo. A mano a mano che egli era piì arvolto nelle spire delli sua sciagurata passione, più diventavauo tornentose ed acri le sue gelosie. E cominciarono le affannose vicente dej dissidii amorosi e delle riconciliazioni. La donna ogni 
rolta che si sentiva sfuggire la sua preda, si scagliava a riprenderla, or giurando di preferire Catullo allo stesso Giove (LXX. LXII), or facendo roti a Venere e a Cupidine di hruciare $\mathrm{i}$ rersi di un pessimo poeta, se Catullo ritormasse a lei (XXXVI). E Catullo si vide passare daranti tutti gli amici di un tempo, che or volerano partecipare con lui ai rolgari abbracciamenti di Lesbia: Alfeno, Gellio, Ravido. Rufo, Quinzio, tutti i compagni delle allegre arventure, e vide Lesbia andare sempre più in hasso per la china delle ignominie, e passare per le mani (lei duecento giovinastri della putida osteria (XXXVIII), a andare pei crocicchi e pei viottoli, spellando i magnanimi nepoti di Remu (L,Ylll). I suoi carmi sono un'eco, forse attenuata, del sno dolore. Ed era naturale che l'animo esulcerato prorompesie al vituperio ed al disprezzo. Un suo acerbo componimento acgressivo ei mostra tutto l'impetr, che scatta, dallanima slla offesa e sanguinante. Ci si consenta qui di riportarlo, nella traduzione italiana (1):

Venite, endecasillabi, jua tutti,

Quanti siete. venite da ogni parte;

Mi prende a grioco quella turpe atultera

E render non $\mathrm{mi}$ vuol le tavolette.

Se voi lo soffrirete. Inseguiamola.

(1) Carme XIVIJ. Ounta trabuionte comm quella whe segue poco lopo, i nostra. Clr. Catullo. I rormi. Tradusiome di Carlo l'ascal e li altri. Milano. Istitute edituriale italiano, 1915. Questa eflizone $\vdots$ inquinata ron un orrilo celfo, prosentato comse ritratto di Catulle. e rhe is inframente lantastieo, "fu messo a mia in-

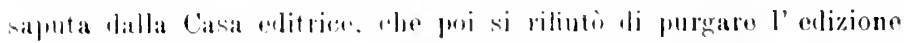

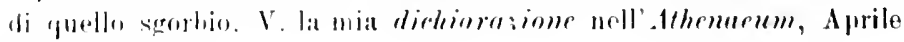
1915, , 209. 
Che le renda. Chi sia cotesta femmina

Voi mi chiedete. Eccola là che incede

Lascivamente, ed a sguaiata smorfia,

Siccome mima, atteggia il labbro e ghigna

Come gallico cane. Circondatela,

Gridate : « i codicilli, o turpe adultera,

Rendi, o adultera turpe, i codicilli ».

Non te ne curi? O fango, o lupanare,

0 s'altro esser vi può di più perduto.

Ma neppur basta. Almen, s'altro non puossi,

Caviamole il rossor da quella bronzea

Faccia di cagna; con più alta voce

Gridate ancora: "rendi, o turpe adultera.

Turpe adultera, rendi i codicilli ».

Ma non si smuove e niente se ne cara.

Se qualcosa si può sperarne ancora,

Convien dunque mutar registro e modo:

"Pudica e proba, rendi i codicilli ».

Ma probabilmente quell' amore sciagurato sopravrisse ad ogni delusione, ad ogni infedeltà, ad ogni onta. Ed il poeta non chiese ormai altra grazia agli dèi, che di esserne libero; e gettò, col piauto nella roce, un grido straziante di disperazione (carme LXXVI):

Se i benefizi antichi son dolcezza all'uom che ricordi,

Quand'ei seco ripensi che serbò puro il cuore,

Che non la santa fede violò, che del nume divino

In nessun patto mai abusò per inganno,

$\mathrm{Nel}$ remoto arvenire t'aspettano molti, o Catullo,

Predestinati gaudii per questo ingrato amore.

Tutto ciò che sul mondo si può fare o dire di bene,

Tutto da te fu fatto, tutto da te fu detto,

Ia ad un ingrato cuore fu rivolto, e tutto fu vano:

Perchè dunque più a lungo teco stesso ti crucci?

Perchè non ti fai forza? Perchè non ritorni a te stesso,

$\mathrm{E}$, se gli dèi non vogliono, d'esser misero cessi?

Dura cosa è d'un tratto un si lungo amore obliare, 
Dura cosa; ma pure convien che tu lo faccia: Questa è la tua salvezza, questa pugna vineer tu devi.

E, n'abbi o no la forza, è questa or l'opra tua.

0 dè, se a voi si aldice pieti, se un ainto supremo

Voi portaste gianmai pur sul punto di morte,

Volgete a me lo sguardo; e se la mia vita fu pura,

Strappatemi dal cuore questo tormento atroce,

Che mi va serpegrgiando per l'ime midolla, o attutisce

Ogni senso, e mi seaceia dal petto ogni letizia.

Nè chiedo io gia che dessa l'affetto mio mi ricambi,

0 , cio ch'esser non puote, che voglia esser pudica;

Solo guarire io voglio, depor quest'orrendo mio male:

Se son vissuto pio, deh, m'esaudite, o dèi : 
APPENDICI 



\section{Elementi rettorici nella poesia Catnlliana.}

I poeti latini, anche i maggiori, come i poeti di qualsiasi altra età o nazione, non potettero mai, in tutto il corso dell' attività loro, liberarsi interamente dagl'influssi e dai caratteri della loro prima educazione letteraria; e la prima educazione letteraria dei poeti romani nell'ultimo secolo della Repubblica fu prevalentemente rettorica (1). Io non sono certo disposto ad esagerare i dami, che da tale specie di educazione vennero alla loro ispirazione individuale ed alla libera formazione del loro genio poetico. Testè anzi di questa specie di educazione e dei suoi effetti su tutta la letteratura latina, si è sostenuta una valida di-

(1) Il Leo (L. Annaei Senecae Tragoediae, vol. I, Observ. criticas continens, Berolini, 1878, p. 148-158) che buone osservazioni fece sulla tragoedia rhetorica, è dubbioso se l'inizio di una tal tendenza letteraria, debba risalire per la tragedia a Vario o a Pollione (p. 148). Per le altre forme poetiche tale tendenza si manifestò però anche prima; e nella stessa poesia drammatica si era manifestata prima nel mimo.

C. Pascal

13. 
fesa. "La rettorica, dice il Cocchia (1), non è in fondo che l'arte del dire, e ne possono disconoscere il pregio soltanto quelli che si ostinano a negare l'efficacia e la necessità dei precetti a meglio raggiungcre $\mathrm{i}$ fini dell'arte. Contro di questa illusione, che prescinde da qualsiasi considerazione storica intorno al cammino dell'arte, basterebbe invocare la persistente tenacia dei motivi tecnici, che presiedono al suo srolgimento, e additano l'opera paziente della scuola nella preparazione del terreno artistico, che il genio feconda ». Certo tal metodo di educazione, che consiste poi in sostanza nel trarre dai grandi scrittori i precetti e gli esempii, affina il gusto, educa il sentimento artistico, c sviluppa in chi tenti ricalcare quelle vie, lo fren dell'arte. Ma nell'educazione rettorica romana vi era tutta un'altra parte, che tendera piuttosto a mortificare gl'ingegni, che ad eccitarne vigorosamente le facoltà; e i cui effetti erano necessariamente d'irrigidire l'arte in forme convenzionali, in schemi fissi, sicchè gli elementi non più ne scaturissero dall'intima commossa personalità del poeta, ma fossero tratti da regole tradizionali, e fossero quindi, appunto perchè costanti, non più vitali energie di arte. Cotali effetti dell'educazione rettorica si ravvisano perfino in Catullo, cioè nel poeta più libero, più spontaneo, più impulsivo della latinità; e non solo negli epillii mitologrici di imitazione alessandrina, bensì anche in qualcuno dei carmi di carattere intimo e personale (2).

(1) Introduzione storica allo studio della letteratura latina, Bari, Iaterza, 1915, p. 22.

(2) Se si vuole aver un esempio dol grado cui puó giungere nella tradizione poetica l'aluerrazione nol seguire un modello con- 
Catullo passò la sua fanciullezza e la sua prima adolescenza a Verona. Era cittì grande e fiorente, che arera l'ambizione di imitare i costumi e il lusso della capitale (1). Catullo stesso, in un passo del carme LXVIII a (vr. 15-16) accenna alla gioconda rita dei suoi sedici anni, quando egli nella nativa Verona dividera le sue giornate tra le occupazioni di Venere e quelle delle Muse. Ivi si formò la sua prima educazione poetica: ivi nella scuola di qualcuno dei retori piǹ noti, egli dovè cominciare ad amare i grandi modelli della poesia eolica ed alessandrina. Era fiorente nella Gallia Cisalpina una scuola di grammatici: Suetonio ci apporta i nomi di Octavius Teucer, Sescenius, Oppius Chares (2). In qualcuna di queste scuole rettoriche si formò l' educazione letteraria del grande poeta reronese. Nelle scuole rettoriche erano principali esercitazioni quelle delle theses e dei communes loci. Quintiliano lamenta che cotali esercitazioni fossero ai suoi tempi un po' trascurate nelle scuole di rettorica, mentre prima ne formavano la parte principale (3). Le

venzionale di arte, si consideri il modo onde celebravano la bellezza femminile i nostri poeti petrarchisti del Cinquecento. Essi, anche quando dichiaravano che la loro bella era bruna, ne celebravano le chiome bionde, e talvolta ne lodarano insieme le ciglia nere e i biondi capelli. Vedi gli esempii raccolti da Fortunato Fizzi, nello scritto intitolato Qua e lì pel Cinquecento. Capelli biondi, in Fanfulla della Domenica, 28 Marzo 1915.

(1) Un po' forse prolisso, ma ad ogni modo degno di particolare menzione è il capitolo I dell'opera di A. Couat, Etude sur Catulle (Paris, E. Thorin, éditeur, 1875), capitolo che s'intitola appunto Catulle à Vérone, p. 20-34.

(2) De illust. gramm. III.

(3) Quintiliano II, 9: "An ignoranus antiquis hoe fuisse ad augendam eloquentiom genus exercitationis, ut theses dieerent et 
theses erano proprio quelle che anche oggi chiamiamo tesi, nel linguaggio didattico, ragionamenti critici, che si svolgono contrapponendo l' una all'altra due proposizioni e vagliaudone le ragioni. Ia sola differenza fra le tesi di allora e quelle di oggi è che, allora, le tesi riguardavano di solito problemi della vita morale, come questi che cita Quintiliano: se sia da preferirsi la vita rustica o la vita urbana: se il giurisperito debba valere più del militare, e simili (1); oggi le tesi riguardano tutti i più minuti particolari in ogni genere di materia scientifica.

I retori insegnavano nelle scuole anche i luoghi comuni. Erano categorie di pensieri, sistcmaticamente disposte, che doverano fornire agli scrittori ilee, immagni ed esempii. Si attingeva di là come da un serbatoio comune: l'espressione di una idea o la menzione di un fatto richiamavano una idea o mna serie di fatti simili, appartencnti appunto a questo patrimonio comune; equesta materia veniva trasmessa dall uno all' altro, un po' variamente foggiata e mutata nelle sue forme, ma, nella sostanza sua, identica. Si trattara di mamante infedele? Ed ecco la descrizione degli Elisii, che aspettan gli amanti

communes loeos et cetera citra complexum rerum personarumque. quibus verae fietaeque controversiae eontinentur? E.x quo palum est quam turpiter ileserat eam partem rhetorices institutio, quam et primam habuit et diu solam".

(1) Quintiliano, II, 4, 22. Altri esempii di tesi frequenti nollo

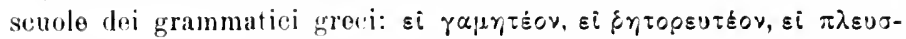

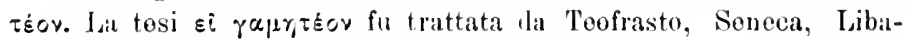
nio ore. Della tesi de rustira rita an mbama si ha 13 accenno, più che uno svolgimento, in Cicorone, Iro Rosc. Am. c. 27. Circa le tosi ofr. Richard Volkmann, llie lihetorili aler Girichen und liïner (lierlin, 1872), \%. 21. 
fedeli, e la menzione delle pene, che aspettano laggiì chi ha violato il nume dell' amore (1). Si trattava di un viaggio marittimo periglioso? Ed ecco le imprecazioni contro chi primo osò commettere alle onde una fragile barchetta e non ebbe paura dell' infuriare delle procelle (2). Nessuno dei poeti è alieno da cotali espedienti convenzionali. E non ne è alieno neppure Catnllo. Ricerchiamoli brevemente (3).

Uno dei luoghi comuni più frequenti presso i poeti latini è quello geografico. Il gusto dei Romani per i viaggi, la loro curiosità per la conoscenza di lontani luoghi, aveva fatto fiorire nel loro linguaggio una gran quantità di espressioni riferentisi appunto alla geografia, ed aveva nella menzione di luoghi lontani, deliberatamente ricercata, una costante manifestazione letteraria. Se Catullo vuole accennare al fervore della sua passione amorosa, ricorre appunto a due paragoni geografici: egli arde quanto la rupe trinacria, o la malia fontana sulle Termopili oetee:

Cum tantum arderem quantum trinacria rupes, Lympharue in oetaeis malia Thermopylis.

(LXVIII, 53-54 = LXVIII b, 13-14).

(1). Cfr. Tibullo I, 3, 57; Properzio IV (V), 7, 59, ecc.

(2) Cfr. Properzio I, 17, 13, IV [V], 3, 19; Orazio, Carm. I, 3, 9. Anche Catullo LXIV, 171.

(3) Parlando dei luoghi comuni non intendiamo naturalmente con ciò esaurito l'argomento dell'influsso dell' insegnamento rettorico sulla poesia catulliana. Rimarrebbe ad esempio a parlare dell'amplificazione rettorica, per cui si presentano come frequenti o come molteplici fatti o personaggi isolati. Un esempio catulliano è LXVIII, 115 [= LXVIII b, 75] : pluribus ut eacli tereretur ianua divis; qui si allude al solo Ercole. Molti altri esempii greci e latini consimili, racolse, dopo il Welcker, il Leo, in L. Annaei Senecae tragoediae, I, Observ. criticas continens, pag. 150, nota 3. 
Se ruole accennare al numero infinito di baci. che egli vuol lare alla sua Lesbia, il paragone ì tratto appunto da conoscenze geografiche: "Quanto ì grande il numero dei granelli di sabbia libica, che giacciono in Cirene ricca di laserpizio, tra l'adusto oracolo di Giove e il venerando sepolcro del vecchio Batto \& (VII. 3-6) (1).

Il tradimento di dne amici, Furio ed Aurelio, gli rammenta l'antica loro promessa di essere sempre insieme con lui, pur se egli si recasse nei più lontani paesi, ed egli swolge con compiacenza questo motivo geografico per tre strofe nelle quali non si può negare che si diluisca l'espressione e nella enumerazione dei popoli più Jontani si raffrerldi l'impeto e si attenui l'energia poetica (2).

La semplice menzione affatto occasionale di Brescia, fa che il poeta aggiunga i particolari sulla città: Brescia che grace ai piedi del colle cicnèo, e il biondo Mella vi passa con languida corrente, Brescia la madre amata della mia Verona (LXVII, 32-34).

In nno scherzoso carme il poeta rammenta un voto della sua fanciulla: quello di dare alle fiamme i carmidi un pessimo poeta, se cssa giungesse a rappattumarsi con Catullo. Egli ora ha trovato questi carmi: sono gli annali di Volusio, carta sudicia; c il poeta si rivolge a Venere, perche faccia pago e gradisca il voto della sua fanciulla; ma la menzione di Vencre tosto grli richiama alla mente

(1) Quam marnus numerus libyssae harenae Laserpiciferis iacet Cyronis.

Oraclum lovis inter astuosi

lit Batti veteris saerum sepuleum

(2) Carme XI, 1-1\%. 
la reminiscenza della scuola, il luogo comune: il ricordo di tutte le località che sono sotto la protezione della dea; ed ecco la lunga enumerazione:

O dea, creata dal ceruleo mare,

Che abiti il santo Idalio, gli Urii porti, Ancona, Gnido ricca di canneti, Dirrachio, emporio dell' adriaco mare, Ed Amatunta e Golgo, accetta, o dea, E fa pieno tal roto....

(XXXVI, 11-16).

Nel carme XXXIX Catullo se la prende con un suo rivale, piì fortunato di lui con Lesbia, Egnazio di Celtiberia. Nella pettegola società dei giovanotti romani aveva molto credito una diceria: che in Celtiberia si adoperasse cioè uno strano dentifricio, l'urina. Naturalmente Catullo coglie la diceria, e ne ha buon gioco per dileggiare Egnazio: se egli fosse di un altro paese, farebbe bene a mostrare i denti: e questo concetto è diluito nel luogo comune geografico: "se tu fossi di Roma o Sabino, o Tiburtino, o pingue Umbro o obeso Etrusco, o nero e sannuto Lanuvino, o Transpadano, per rammentare altresì i miei concittadini, o di qualsiasi altro paese.... ».

$\mathrm{Ci}$ si consenta di riportare da una nostra recente traduzione tutto il pungentissimo carme:

Egnazio perchè candidi ha i denti, si fa bello In ogni evento a ridere. Dinanzi allo sgabello Del reo, ruando le lacrime eccita il difensore Ei ride. Innanzi al funebre rogo d' un casto giovine, Quand'orba madre l'unico piange perduto amore, Ei ride. Per qualsiasi cosa, dovunque sia, Qualunque cosa facciasi, ei ride. È malattia Non certo, a quel ch'io giudico, né garbata nè fine. 
Laonde, o bravo Egnazio, senti due paroline.

Se tu avessi per patria Roma o Sabina o Tivoli,

Se fossi Umbro ben turgido, se Etrusco ben pasciuto,

Se fossi di Lanuvio, color moro, e sannuto,

0 Traspadan (per essere memore pur dei miei),

0 fossi onde che siasi, dove peraltro s' usino

Puliti dentifricii, però non gradirei

Vederti ognora al ridere atteggiare il tuo riso

Chè niente v'è piǹ stupido di uno stupido riso,

Ma sei di Celtiberia: e in terra Celtiberia

Ognun fregare è solito con la notturna urina

Le gengive purpuree e i denti ogni mattina.

Appare dunque nitido il vostro dente e liscio:

Vuol dir che in maggior eopia beveste il rostro piscio (1).

Questo procedimento tecnico dovera essere particolarmente grato a Catullo, pieno, fin dalla giovinezza prima, dello spirito di aventure, e che si era anch'egli cimentato in lunghi e perigliosi viaggi, e che ritornato alla sua tranquilla Sirmione, sciolse un carme al burchielletto che In avera portato da mari così reconditi sino al suo limpido lago. Anche questo carme contiene enumerazioni geografiche (:) , ma in esso non si tratta darvero di luogo comune, bensì di vera vita vissuta.

(1) Catullo, I curmi, traduzione di Carlo Pascal e di altri, prefazione di Carlo Paseal. Milano, Istituto editoriale italiano, 1915, p. 66.

(2) IV, 6-13. In altro carme l'evocaziono del praesaggio geografico è li grande efficacia pittorica. F̀ il carme LXI, l'epitalamio di Giunia e Manlio. Imeneo deve affrettarsi a congiungere $i$ voti lesiderati. abbandonando gli antri aonii della tespia rupe, cui la ninfa Aganippe rinfresea dall'alto irrigandoli : "Catulle distribue heureusement dans la strophe les trois nomes géographiques de Thespies, l'Aonie et "l'Agranippe. Nous avons iri sous les yeux un rlarmant paysage: les roches, une grotte, l'eau tombant en cascalle l'une source qui la rafraichit: super fait image (Patin). 
Altro luogo comune della poesia Catulliana è quello mitologico. In un poeta studioso degli Alessandrini, ed anzi amatore ed anche traduttore di Callimaco, è naturale aspettarsi un largo uso della mitologia. Il romano Callimaco, cioè Properzio, ne fece anzi uso larghissimo: il mito forma quasi l'ossatura di ciascun suo componimento elegiaco, e posto nel mezzo di tutto il componimento dovrebbe nella mente dell'autore dare conferma, illustrazione e rilievo al sentimento onde l'elegia muove. Ha il continuo richiamo ad esempii mitici ingenera una impressione di freddezza e di convenzionalità, che nuoce ai fini dell'arte. Catullo è molto più sobrio. Egli richiama i miti, per colorire con qualche immagine o precisare con qualche paragone il suo pensiero. Che il richiamo sil sempre felice, non si può asserire: ma ad ogni modo non è cosi ricercato e così deliberatamente costante, da inquadrare la poesia in uno schema fisso, come appunto arviene in Properzio. Ed ecco dunque i principali di questi accenni mitologici.

Catullo va in traccia, è detto nel carme $L V$, dell'amico Camerio, che si sottrae agli amici per dedicarsi interamente agli amori di donzellette e rimanere nascosto nel loro seno. Na è difficile impresa il rintracciare Camerio! Il poeta deve porre in rilievo come sia difficile, e ricorre al serbatoio mitologico, che di difficili imprese era ben rigurgitante:

Ma riportarti è una fatica d'Ercole

Tanto altezzoso è il tuo diniego, o amico. 
Non se custode dei Cretesi io fossi,

O sul caval di Pégaso volassi,

E non se fossi Lada o Pérseo alipede.

Non se di Reso sulla nirea biga

Corressi celere, oppur se mi portassero

Pinnipedi ed alati e venti, insieme

Congiunti, saria facile l'impresa.... (1).

Non si può dire darvero che tutto il concetto acquisti nitore da questo affastellamento di richiami mitologici.

Per significare cosa assai gradita che tocchi ad alcuno, dopo lungo desiderio, Catullo rammenta (II, bis) quanto fu gradito ad Atalanta, la veloce fanciulla, l'aureo pomo per eui primamente fu sciolta la sua zona verginale. Nota i la favola di Atalanta, che vineeva tutti nella corsa, ed era stata promessa dal padre a ehi la rineesse: Ippomene col favor di Venere, le gettó davanti aurei pomi, ed essa per raccoglierli fu superata in velocita (efr. Ovidio, Metam. X, 560).

Il pianto assiduo per la morte del diletto fratello anche gli suggerisce un paragone mitologieo: quello di Filomela, che geme per il fato del suo caro ltilo, ed anche trasformata in usignuolo, sotto le dense ombre dei rami, continua il suo remito (LXV, 12-13).

Pieno di richiami mitolongici è il earme LXVIII, che i) il piu vieino, per tal rispetto, al tipo che era stato

(1) La traduzione i nostra (Catullo, I Carmi, Nilano, 1915, 1. 78). Poniamo questi versi, seconlo il Froohlich o lo Sehwabe, dopo il r. 14 rel carme LV; altri dopo il v. 13 (editio princeps ( Lachmann), altri lopo il 12 (Coul. parig. s236 o (iuarino). Vi fu chi vide in fuesti versi il frammento di un secomdo carmo a Camoris. Il Frank (Americen Journal of Ihiloloy!y, 1914) una redazione rifintata dal poeta stesso del arme IV. 
alessandrino, e che poi fu dell' elegia properziana (1). L' occasione a tali richiami è duplice: la morte del fratello, che giace laggiì nella lontana Troade, sepolcro comune dell' Europa e dell' Asia, e la felicità goduta da Catullo insieme con Lesbia, nella casa ospitale di Manlio. Lesbia che sfiora col fulgido calzare la soglia ospitale gli appare quale Laodamia corrente all' amplesso dell' amato Protesilao. Ma il ricordo di questo amore infelice fa sì che per lungo tratto (LXVIII, 73-88=LXVIII b, 33-48) ei s'indugi a narrarne i tristi eventi. Indi il j'icordo che Protesilao morì a Troia, ore è anche sepolto il fratel suo, fa ch'ei torni al ricordo della spedizione troiana e di Paride (LXVIII, 101-104= LXVIII b, 61-64), e poi di nuovo a Laodamia (ivi, $105-108=65-68$ ). Ma ecco innestato al mito di Laodamia un altro mito; e il pretesto per innestarvelo ì una parola, la parola barathimm; vien fatto di pensare a quei processi tecnici per il collegamento

(1) Giustamente il Lafaye. Catulle et ses modèles (Paris, Hawhette, 1904, p. 209): "Si l'on excepte la piêee LXI', manifestcment traduite de Callimaque, il n'y en a pas, dans tout le vecueil de Catulle, où se montrent sous une forme plus apparente les proeédés de l'école alexandrine». Indifferente ai fini di queste nostre osservazioni è la questione se nel carme LXVIII si abbia una unica elegia (v. ad es. tra gli altri W. Hörschelmann, De Catulli carmine LXVIII eommentatio, Dorpat, 1889), o due (cfr. Franz Hermes, Beiträge (1888) e Neue Beitrüge (1859) :ur Kritik und Erklärung des Catull, Progr. Frankfurt, 1888-89). Sulle varie questioni che si connettono a questo carme cfr. anche Theorlorus Birt, De Catulli ad Mallium epistola (Ind. lect A vad. Marpurg.) 1890 ; Alph. Kalb, De duoseptuayesimo earmine Catulli (Progr. Gymn.) Ausbach, 1900; Frank, A rejected poem and a sulstitute. in The American Journal of Philology, XXXV, 1914, p. 67-73; Fr. Guglielmino, in Athenacum, 1915, p. $426 \mathrm{seg}$. 
dei miti, che furono poi così familiari ad Ovidio nelle Metamorfosi. Il haratro in cui cadde Laodamia fu così profondo come fu profondo quello che scavo Ercole, mediante canali ai piedi del Cillene, canali che fecero prosciugare quel suolo reso malsano dalle paludi. Per tali fatiche si apri ad Ercole la porta del cielo, ed Ebe non visise colà vergine eterna. La comparazione. che comprende otto versi (LXVIII, 109-116 = LXVIII b, 69-76) non c̀ delle più felici: certo non agrgiunge nessuna linea e nessun colore alla rappresentazione della redorata Laodamia, assorta nel suo cupo dolore: che cosa ha che fare col dolore di Laodamia questo ricordo di una impresa di Ercole: Liunico pretesto di collegamento è la parola barathrum, arloperata a denotare l'abisso di sventura in cui cadde Laodamia; gracchè baruthrum

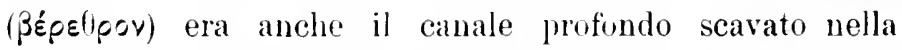
montagna. Evidentemente Catulo quando riproduce dagli alessadrini non is nè caldo poeta nè efficace dipintore. E che per questo passo egli abbia avuto dinanzi un modello di tal genere is più the probabile. Gli alessandrini ricorrevano appunto a questi mezzucci di arte. E ad un modello greco sembra anche accennare l'aggettivo falsiparens riferito al Amphitryoniades, e che ì traduzione

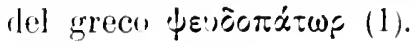

(1) Il Baelırens (I)ie Laodamia-siage und Catullus bs Gedicht, in Vewr Jahrbiecher fier Philologie, 1sit, p. 409 e 105 is pensa appunte who nellelegia IXXVIII Catullo imito qualche opera alessandrina ora perduta; o crede non improhahile cho il molello sieno

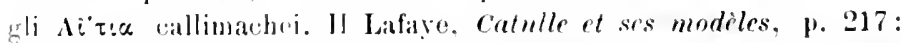
" tont er que l'on peut lire rest que, danse ec moreran, il a peutitre, en flassant. mis if profit les somemirs que lwi araient laisses ales rlegingues alerandrims. 
Ma dopo l'impresa di Ercole ritorna il discorso a Laodamia e ne celebra lappassionato e ferrido amore per il biondo suo sposo. Laodamia è bella e gentile come la sua tanciulla. che grli si è abbindonata fra le braccia, mentre Cupido candido in rolto e fulgido nella tunica color di croco le danzava d’intorno. Ya. ahimè, il ricordo della sua fanciulla gli suggerisce un altro paragone mitologico, che dorera essere molto duro al suo cnore d'innamorato. Lesbia non è contenta del solo Catullo. ma egli sopporta i suoi rari furti damore. per non direnirle troppo molesto: anche Giunone. la massima delle dee. dorè spesso reprimere lira prorompente ai numerosi furti di Giore. il suo onnirolo marito (ivi, $138-140=9-100$ ): Era, purtroppo. onnivola anche Lesbia. e Catullo lamenterà tra non guari i suoi trecento adulteri (XI. 18) e i duecento dirudi della sozza osteria ( $\mathrm{XXXY}$, i-s).

Non ci occupiamo naturalmente qui nè dell epitalamio di Peleo e Teti (carme LXIV) né della Chioma di Berenice (carme LXYI). si perchè la mitologia in tali carmi non è luogo comune. bensi è l’argomento stesso del carme. si perchè. molto probabilmente il primo e certamente il secondo, sono traduzioni di esemplari greci. solo. nel primo di essi rilereremo un passo. che appartiene alla geografia mitica. e che. per la forma e il contenuto costituisce un motiro poetico comune. LXIV. 154:

Quaenam te genuit sola sub rupe leaena.

Guod mare conceptum spumantibus exspuit undis.

Guae Srrtis. quae Scrlla rapax. quae rasta Chargbuis:

Questo motiro fu usufruito anche altrore da Catullo. LX. 1 : 
Num te leaena montibus libystinis.

Aut Scylla latrans infima inguinum parte

Tam mente dura procreavit ae taetra....

e troval un'eco nella poesia di Ligdamo (ps. Tib. III, 4, si) :

Nam te nee vasti genuerunt aeruora ponti,

Nee liammam volvens ore chimaera foro,

Ner canis anguinea redimitus terga caterva,

Cui tres sunt linguae tergeminumque eaput,

Seyllaque virgineam cauibus succincta figuram.

Nec te conceptam saeva loaena tulit,

Barhara nee Scythiae tellus horrendave Syrtis (1).

$$
\text { ** }
$$

Or qui si presenta unaltra questione: quali sono i luoghi comuni della poesia amorosa, che si ritrovano in Catullo: (2) Fortumatamente qui la mèsse è molto povera: la forza della passione e della grelosia fa prorompere dall'anima del poeta le sue parole di amore e di dolore, e non wi laseia modo di pensare nò a dotte reminiscenze

(1) Il passo di Ligdamo i imitazione del primo passo catulliano contaminato col secondo. Cfr. Antonius Danysz, De scriptorum imprimis portarmm liomanorum studiis Catullianis, Posnanias, 1876, p. 16.

(2) Non ci occupiamo naturalmente delle similitudini e delle figure frequentissime nel linguaggio della poesia amorosa, per le quali ha un buono e ben documentato eapitolo il Pichon, De sermone amatorio apud latimos elegiarmm seriptores, p. 18-26. Tali sono lo espressioni commissime di fuochi, fiamme, fiaccole, ali di amore, saette di Cupidine, tutte quelle tratte dal paramone dell'amore con una milizia, "moltissime altre. 
nè a motivi conrenzionali (1). Ad ogni modo notiamo: il concetto che la donna è mobile (praecipue multivola est mulier, dice Catullo LXVIII, $128=$ LXVIII b, 88), e che i renti si portino i suoi giuramenti e i suoi pianti è comune

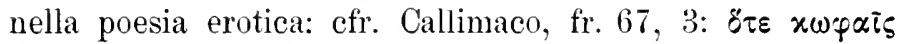

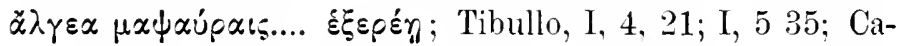
tullo LXIV, 164-166; LXX, 3-4 «Dicit, sed mulier cupido quod dicit amanti, in vento et rapida scribere oportet aqua (2)».

La protesta che si voglia l'amore a qualunque costo, malgrado il disonore, malgrado il tradimento, si trova frequente in Properzio I, 8, 26; I, 12, 19-20; I, 15, 32 * Sis quodcumque voles, non aliena tamen! »; II, 32, 60. Ovidio ha, Amores III, 11, 45 «quicquid eris, mea semper eris ». Questo sentimento trova accenti profondi ed - accorati in Catullo: LXXII, 5-6; LXXV, 3-4; LXXXV, 1-2.

Il giuramento d'amore per gli occhi proprii o la dichiarazione che la persona amata sia più cara degli oc-

(1) Nolto bene il Couat, Etude sur Catulle, p. 169: «Les defauts de la eomposition sont moins ehoquants dans les piéces amoureuses ou politiques de Catulle, et eela pour deux raisons. Elles sont d'une brièveté qui ne permet pas le désordre, et l'émotion du poète en fait l'unité. Son esprit peu étendu et plus vif que profond s'y trouvait mieux à son aise " ecc. p. 171 : "Ainsi se sont échappées de son coeur des pièces passionées,... ainsi il a écrit ces vers si simples où, obéissant à une joie sincère, à une douleur profonde, il a trouré sans eonvention et sans recherche la composition la plus forte et la plus saisissante, celle qui résulte de l'unité et de l'ardeur du sentiment qui inspire le poìte».

(2) Cfr. R. Pichon, De sermone amatorio apud latinos elegiarum scriptores (Paris, 1902, p. 289): "Ventis saepe comparatur cursus amoris, Ovid. Ars. am. III, 584; Rem. 14, 531. Ventosus dieitur Amor, quasi inconstans: Orid. Am. II, 9, 49 . 
chi proprii sono frequenti motivi della poesia erotica (1). Cfr. Properzio, I, 15, 33-35: « ocelli Per quos saepe milhi credita perfidia est! Hos tu iurabas » ecc.: Ovidio, Amores II, 16, 43: " iuraras Per me, perque oculos, sidera nostra, tuos »; III, 3, 9; III, 3, 13; III, 11, 48; Ligdamo 6,47 "perque suos fallax iuravit ocellos» (Ps. Tib. IV, 5, 8). In Catullo amare piu degli occhi proprii è consueta espressione, riferita al passero di Lesbia (III, 5), o all'amico Calro (XIY, 1), o alla domna amata (LXXXII, 3; CIV, 2). L'espressione è tolta dal linguaggio popolare; c si trova infatti nella commedia (Terenzio, Adelph. 701, $903)$ e nella satira oraziana (Sat. II, 5, 35) (2). Cfr. anche in greco, Teocrito, XI 53 bे tepov ousév; Hosco, Idill. III, 9; Callimaco, Inno a Dialea, 211 .

E comme che i poeti inmamorati consiglino di godere la vita, finchè ci è dato. Ufr. Properzio II, 15, 23-24; III, 7. 2.3-2.t « Dum nos fita sinunt, oculos satiemus amore; Nox tibi longa renit nec reditura dies»; 1, 19, 25 «Quare, dum licet, inter nos laetemur amantes»; Tib, I, 5, 69-70 «In-

(1) Cfr. Renó Pichon, Ite sermome amatorio apud latinos clegiarum seriptores, Paris, Hachette, 1902, P. 219.

(2) Il carattere popolare dell'espressione è assicurato dall'uso dol smperlativo oculissimms por "earissimo'. Cfr. P. Pierrugues, Glossarium eroticum linguae latinae, p. 356: * Simili modo Galli: Je t'aime comme mes yeux. - Salce, oculissime homo. Plaut. Curcul. 1, 2, 2-s. Sie et alibi neulitus amare, id est valde et singulariter ". Oculissimus i in Plaut. Cure. 15. 16, 121; cfr. Festo 178, 21 e laolo ex Fest. 179. 8. Per oculitus efr. Nonio, 147, 27 (won eitazione del frammento di Planto \& Qui amant ancilam meam Iledulinm oculitus s), e Frsto 17S M. (1SS Lindsay) : " Oenlitus licitur ut fumlitus, penitus, ienilieatgue tam carmo esse quam oculum, vel instar ounli esse 
terea, dum fata sinunt, iungamus amores, Iam reniet tenebris Mors adoperta caput »; Varrone presso Nonio 156 "properate Vivere puerae, quas sinit aetatula, Ludere, esse, amare et Veneris tenere bigas ». Questo motivo poetico dettò a Catullo accenti ispirati: cfr. il carme $\mathrm{V}$ "Vivamus, mea Lesbia, atque amemus» e LXI, 233 "bene vivite et Iunere assiduo valentem Exercete iuventam ».

La superba affermazione della propria gloria poetica e della immortalità, cui i poeti consacrano le persone cantate, è in tutti gli elegiaci latini. Essa non è per taluno di essi un luogo comune; è motivo poetico, che prorompe da intima orgogliosa coscienza; cfr. Properzio, I, 11, 7-8; I, 15, 24; II, 24, 1; Tibullo, I, 2, 65: "Quem referent Musae, rivet»; Ps. Verg. Catalept. IX, 23-24. Per Catullo redi LXXVIII, 10 "Te omnia secla noscent »; e LXVIII, 41-48 = LXVIII b, 1-8.

E cosí pure dall' intima coscienza artistica di questi poeti scaturisce l'affermazione, che è in essi frequente, che la gloria verrà ad essi dai piccoli componimenti poetici, non dalle grandiose intraprese epiche; cfr. Properzio, I, 7, 9-14; 11arziale, VIII, 3; IX, 50. Per Catullo cfr. I, 10 e XCV, 6-10.

Non sarebbe difficile forse continuare in altri riscontri. Ma anche da quelli fatti si scorge qual sia il carattere precipuo della poesia amorosa di Catullo. $\grave{E}$ in genere una poesia spontanea, libera, prorompente dal sentimento acceso, ignara di formole tecniche, di schemi fissi e di procedimenti convenzionali, nelle cui strettoie debba svolgersi. Pensieri comuni ci sono, ma derivano dagli elementi comuni a tutte le passioni di amore, dagli atteggiamenti consueti che tal sentimento suol prendere negli

C. Pascal 
animi unani; non derivano da regole inseguate nelle scuole o da precetti rettorici: non sono, in una parola, luoghi comuni. L'unica elegia fatta sul modello delle elegie alessandrine è la LXVIII, che ridonda di elementi mitologici. Ma ben più che per essa e ben più che per i poemetti di soggetto mitologico e di imitazione alessandrina, Catullo è grande, per quei brevi carmi di amore e di dolore, che sembrano altrettante grida dilaceranti di un'anima in pena. 
II.

\section{Orazio e Catullo}

Come è noto, nella Sat. X del Iibro I, Orazio giustifica il concetto che egli ba di Lucilio e le critiche fatte nella Sat. IV. Ma l' assunto dell'autore si allarga a molto più ampia serie di critiche e di fatti letterari; giacchè egli di tratto in tratto tira, quasi occasionalmente, frecciate contro l'uno o contro l'altro. E poco dopo il principio della satira si ha un' acerba punta contro Calro e Catullo (v. 16 e segg.):

IIli, seripta quibus comoedia prisca viris est,

Hoc stabant, hoc sunt imitandi; quos neque pulcher

Hermogenes umquam legit, neque simius iste

Nil praeter Calvom et doctus cantare Catullu

Orazio parla qui dei poeti dell' antica commedia, ed è evidente che egli, mentre consiglia l'imitazione di quelli, se la prende con coloro che altro non sauno fare che recitare Calvo e Catullo, $i$ patiti di Calvo e Catullo, diremmo noi. Già questa contrapposizione è significativa: 
se ̀̀ segno di buon gusto imitare gli uni, è aberrazione imitare gli altri. Calvo e Catullo si trovano congiunti nei medesimi elogi da Properzio, II, 25, 4; 34, 87-89 e da Ovidio, Amores, III, 9, 62; e qui in Orazio si trovano congiunti nello stesso attacco. Ambedue avevano scritto elegie amorose ed acri cpigrammi politici, ed epillii mitologici; ed inoltre eran legati di salda e fida anicizia e di consentimenti politici. Anche il cantare ha valore certamente deprezzativo. Esso ci richiama al pensiero i cantores Euphoriomis di Cicerone (1). Come questo è detto per esaltare Ennio di fronte ai seguaci della nuova scuola poetica, e cioè a Catullo ed ai poeti catulliani, cosi il cuntare di Orazio è letto per esaltare i poeti dell'antica commedia di fronte alla medesima scuola dei poeti catulliani. Non che la poesia catulliana si contrapponesse direttamente all' antica commedia; ma ad Orazio ogni occasione è buona, per seaglane i suoi colpi; e del resto negli epigrammi aggressivi e satirici di Catullo, Orazio potera ben redere in certo modo m riflesso della commedia antica. Ma, come si è detto, la critica oraziana su Lucilio si allarga, quasi per spunti occasionali, ad altri poeti. 'Troviamo lungo il corso della satira attaccati con frizzi più o meno mordaci furio Bibaculo, cui il poetat allude sotto il nome di Alpinus, Varrone Atacino, Laberio, l'antilio, P'itoleone, ehe mescolara il greeo al latino, Casio Etrus(o, un improvrisatore inesauribile, Fannio, m poeta popolare, Ermogene e Demetrio, che sono appunto i recitatori di Calvo a Catullo. Il poeta più rolte

(1) Tuscul. III, 45. Cfr. Collignon, La evitique et les querelles littéraires a kome, p. 21. 
insiste nel ricordare l'uno o l'altro dei due (1); la ripetizione può mostrare che essi non gli erano indifferenti, e che egli dara la debita importanza alla scuola catulliana, di cui quei due si facerano fautori e diffonditori. É stato osservato come Laberio, Catvo, Catullo, Pitholaus (il nome vero adombrato sotto il Pitholeon oraziano?), Bibaculo, erano in origine anticesariani; e si è creduto che alla critica aggressiva di Orazio non fosse estranea la preoccupazione politica. Siccome Calvo, Catullo e Bibaculo, come risulta da Suetonio, si riconciliarono poi con Cesare, bisognerebbe conchiudere che Orazio volesse essere più cesariano di Cesare; il che non pare ammissibile. $\mathrm{E}$ si può anzi osservare che pure la famosa ode nella quale Orazio rammenta Filippi e la relicta non bene parmula è diretta ad un suo amico (2), che era stato fieris-

(1) Versi 18, 79, 80 e 90. Demetrio è nel passo oraziano indicato con simius iste, e simius indica la bruttezza in opposizione a pulcher, ed ha anche l'allusione allo scimiotteggiare Catullo e Calvo, cioè imitare servilmente; significato che è documentato in latino da un esempio di Seneca il retore, $\theta$ che ha riscontro nel pari significato di $\pi i \eta \eta$ ros greco: cfr. la nota del Lejay (IIorace, Satires, Paris, Ilachette, 1911, p. 266). Scol. Crucqui, a q. I.

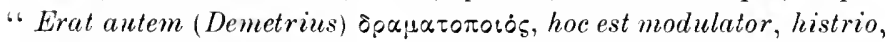
actor fahularum, Ermogrene Tigellio è citato da Orazio anche in III, 129, come cantor et modulator, parole onde forse è desunta l'informazione di Acrone, a q. I. : "Erant autem (Hermogenes et bemetrius) musici modulatores, ,. Orazio qui lo chiama pulcher, e certamente con allusione oscena, come Latullo (LXXIX, 1) dice di Lesbio, come Cicerone dice pulchellus puer (Ad. Att. I, 16, 10); efr. Servio, ad Acn. III, 119: "Pulchros a reteribus cxolctos dictos ,..

(2) Carm. III, 7. Circa il vero significato di non benc efr. il mio articolo in Bollettino di Hilol. classica, Marzo 1909 ; e cirea 
simo ed accerrimo nemico di Augusto; ed Orazio non si fa punto ritegno di scrivergli in termini di affettuosa ricordanza. Prescidendo dumque dalle preoccupazioni di carattere politico, è da esaminare l'arversione di Orazio a Catullo ed a Calvo esclusiramente sotto il rispetto letterario. Ma a questo punto è opportuno fare osservare come malgrado questa aversione, dichiarata e quasi ostentata in forma dispresiativa, risulta in modo indubbio che più volte Orazio imitò Catullo. Giorerà apportare qui le principali di tali imitazioni, per qualche considerazione cui esse si prestano (1).

Esaminiamo anzitutto il carme secolare di Orazio, $\theta$ propriamente la prima parte di esso, quella riguardante la preghiera rituale: giacchè la seconda parte, dal verso 48 in poi, celcbra propriamente le imprese e le guerre di Augusto. Ma nella prima parte invece si ha l'invocazione ad Apollo e Diana, e la celebrazione del loro potere divino. Ora il carme XXXIV di Catullo procede secondo il medesimo schema: anche ivi si celebra Diana, e s'invitano i grovani e le donzelle a cantarne le lodi; manca naturalmente, per l'occasione diversa, la parte riguardante Apollo. Ha pur nei singoli particolari v'è qualche somiglianza tra i due carmi nelle espressioni. Dice Catullo, nel v. 9: "Montinm domina "t fores silvarmmque virentimm »: dice Orazio nel v. 1: "Silvarmmine potens Diann »; Catullo v. 3: "INanam pmeri integri, Pnellaeque ramamms »; Orazio r. 6: "Virgines lectos pue-

la pretesa viltà adulatoria di Grazio cfr. Ernesto Anzalone, Appunti oraziani. Castrogiovanni, 1903.

(1) Cfr. Antonius Danysa. De scriptorum imprimis poetarum romanorum stuliis r'atulliomis, Posnaniae, 1876, 1. 10-15. 
rosque castos... Dicere carmen 》; Catullo v. 13: "T" Lucina dolentilus. Inno dicta puerperis »; Orazin r. 13: "Rite maturos aperire partus Lonis, Ilithyin, tuere matres, Sire t" Lueina mobas cocuri Sen Genitulis »; Catullo v. 22: Romulique... sospites ope gentem »; Orazio r. 47: "Romulae genti date remque prolemque Et decus omne".

Il carme $I$ di Catullo è ispirato al pensiero della fugacità delle cose terrene, e della opportunità di cogliere i piaceri che la vita ci apporti; pensiero che occorre frequente in Orazio, e certamente non per imitazione esclusiva di Catullo. Ma in qualcuno dei carmi oraziani ove tal concetto ritorna, è dato ravvisare qualche riscontro, che forse non è casuale. con Catullo. Dice Catullo, v. 3 e segg.: Soles occidere et redire possunt; Nobis cum semel occidit brexis lux, Nox est perpetua una dormienda. Dice Orazio nel carme VII, libro IV, v. 14 e segg.: «. Vos ubi decillimus Quo pius Aeneas... », e poco dopo v. 21: "Cum semel occideris.... .

Anche la famosa ode catulliana a Lesbia, tradotta da Saffo, sembra essere stata innanzi al pensiero di Orazio quando scrivera l'ode a Jalage (I, 22). Dice Catullo (LI, 4): "te Spectat et audit Dulce ridentem; dice Orazio, (v. 23): "Dulce ridentem Lalugen amabo».

Molto conclusivo è il rapporto tra il carme XLII di Catullo e l'eposlo XVII di Orazio. Ed anzitutto il rapporto è di procedimento; giacchè nell'uno e nell'altro si passa in modo ironico dalle buone alle cattive maniere e dopo avere inveito con atroci contumelie contro una donna, si tentano sareasticamente le blandizie.

Jia oltre questo carattere generale, anche qualche particolare di espressione corrisponde; cfr. all esempio it 
v. 24 di Catullo: Pudica et proba, redde codicillos » col v. 40 dellepodo oraziano: «Voles sonare: tu pudica, tu probas.

Altri riscontri qua e là si possono indicare. Ad es. il procedimento del carme IV, 12 di Orazio rammenta quello del carme XIII di Catullo, almeno in qualche punto (1): nell' uno e nell'altro si tratta di un invito a cena, ed il poeta prega il convitato di portarsi seco qualche cosa: Orazio $u$ p piccolo vasetto di nardo, Catullo molto di più, arldirittura tutta la cena, ma in cambio l'uno e l'altro promettono letizia di godimenti. E questo medesimo carme XII del libro IV oraziano, rammenta nel principio anche il principio del carme XIVI di Catullo. Si vegga infatti (r. 1 e segg.): "Inm reris comites, quae mare temperant Inpellunt animae lintea Thraciae, Iam nec prata rigent nee flurii strepmut »; Catullo (XXXXVI, v. 1 e segg): "Iam ver egelidos refert tempores, Inm caeli furor aequinortialis Iocundis Zephyri silescit anreis (2).

Questi passi mostrano che il carattere della imitazione oraziana da Catullo non è di gran lunga diverso da quel che sia l'imitazione di Orazio da Alceo o da Archiloco.

Questo carattere fu ben determinato da Luciano Müller

(1) Yuesto motivo catulliano fu anche indicato dal Belling, Studien iber dio Liederbieles des Ioratius, Berlin, 1903, p. 119.

(2) Si noti anche: Catullo IXXXYI, 1 Quintia formosa est multis, mithi enndiln, lomyu. lieetu est... ; Orazio, Sat. I, 2, 123: "candida restaque sit, mmmla hartenus ut neque longa, Nec magis alba relut quam dat matme rideri ,, C Catullo VIIl. II : "obstinota mente perfer, obilura ., ; 1)razio, Sat. II, 5, 39: "persta utque obdura, ,; Catullo, IXII, 49: " It ridua in mudo ritis quac mescitur aren,,; Orazio, Carm. IV, 5, 30: "ritem viduas ducit ad arbores ,.. Veli Danysz, op. eit., 1. 14. 
nel breve, ma succoso saggio da lui composto sopra Orazio (1). Questi non traduce odi intere: prende dagli antichi modelli spunti e motivi poetici; talvolta i versi d'introduzione di Alceo e di altri lirici offrono al poeta la materia e la ispirazione per tutta mn'ode; tal'altra i versi del poeta antico son riprodotti in forma più o meno variata in un nesso di pensieri diverso. Tale, a un dipresso, benchè naturalmente in proporzione molto minore, è il modo onde Orazio tratta ed usufruisce i carmi catulliani; ma la differenza è solo in questo, che mentre Orazio riconosce tutto quel che deve ad Alceo e ad Archiloco, per contro, non solo nulla riconosce a Catullo, ma lo tratta come abbiam visto, con un'aria di superiorità e quasi di dispregio.

E questo appunto ci può spiegare la ragione del suo atteggiamento, che cioè a lui tornava molesto il pensiero che altri lo avesse preceduto nei campi, nei quali egli voleva regnare sovrano: e gli contendesse quasi nel giudizio dei contemporanei e dei posteri il vanto della priorità. Ciascuno infatti rammenta come appunto di questa

(1) Lucian Müller, Quintus Horatius Flaccus, Eine literarhistorische Biographie, (Leipzig, Teubner), p. 111-115 (Trad. ital. di Grov. Decra, Firenze, Sansoni, p. J13 sg.) Poche ma giuste idee su tale argomento esprime anche Onorato Occioni, nel vol. La vita e le opere di Q. Orazio Flacco, Bologna, 1893, p. 141 ed ivi nota. Tralasciamo di citare i molti altri scritti in proposito, che si possono del resto vedere nella Bibliotheea scriptorum classicorum del Klussmann (gli scritti posteriori al 1852). I luoghi dei poeti greci (e latini), che hanno riscontro coi luoghi oraziani, sono notati a piè delle singole odi nella edizione di Otto Keller (Q. Horati Flacci Opera, vol. I, Carminum libri, iterum recensuit Otto Keller, Lipsiae, Teubner, 1899). 
priorità egli si facesse ripetutamente vanto. Sel carme XXX del lihro III egli dice aver primo ridotto ad itale melodie il carme eolio: I'rinceps deolimm rarmen ad Italos Dednrisse modos: e nell'epistula XIX del libro I ripete la medesima lode a se stessio non solo per il carme eolio, bensì anche per i giambi archilochei (v. 23 e seg.): Parios ego primms iambos Ostendi Lation. mmeros animospue serntus Archilorhi, non res et ayentia rerba Lycamben. Ac ne me folies ideo brevioribns ormes. Qnod timui mutare modos et rarminis artem: Temperat Archilochi musam pede mascula Siappho, Temperat Alcaeus, sed reLns et ordine dispar ». Ora appunto Archiloco, Alceo e Saffo erano stati importati nella poesia latima non primamente la Orazio, hensi da Catullo: e il poeta ha il torto di non farne un franco riconoscimento. Nè sembri strano che an Orazio si attribuisca l'intenzione malevola di ma cosi rleliherata ingiustizia e la ranità di far suo un ranto ele reli sapera spettare ad un altro; e pegrein ancora di usufruire l'arte di quest altro, pur dissimulamblo ed astentamdo dispregrio.

In rerita di cotali piceole vanita, le quali non sempre son fatte con candido animo, nom puì dirsi affatto immune (Tazio: Vauita i, ad exompio, laccenno che troviamo nellode terza del hiho IV, che cioe egrli sia mostrato a dito dai passanti qual cantore della romana lira che egli debba riconoserele alla musa tal benefizion vanita è l’altro acremno che tmorimo in fine dellode sesta del libro 11, ove il preta, rivolgendusi alla rergine cantatrice le predice che quande estia salua sposa, si vanterà di aver cantato il carme del valre (Marion.

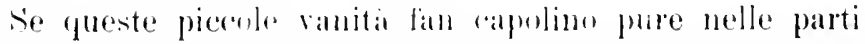
dei cami, ove piu mohile on ispirata e l'exaltazione poe- 
tica, non è da meravigliarsi che se ne riscontrino pure simili o maggiori nei giudizi o negli atteggiamenti critici dell'autore; e questo infatti ptio dirsi non solo per quel che riguarda Catullo, bensi anche per tutti gli altri poeti antichi: Orazio fu troppo fedele al suo precetto di assumere l'orgoglio dell'opera sua, e la sua superbia benchè quaesita meritis passò qualche volta il segno e lo fece ingiusto verso i predecessori. 



\section{NDICE}

Al lettore . . . . . . . . . . pag. $\quad$ p

Averteña . . . . . . . . . . .

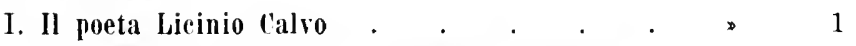

Avvertenza p. 2. Calvo salopugium disertum p. 3.

L' intimità tra Callo e Catullo p. 5. Calvo e Catullo nella tradizione letteraria p. 6 . Un carattere artistico comune ai due poeti p. 9. I poetae docti p. 10. Exiguus Calvus p. 14. I famosa epigrammata di Calvo contro Cesare p. 16. Epigrammi contro Pompeo p. 19. Contro Tigellio p. 23. Contro Manio Curio p. 25. Vatinio e l' odio Vatiniano p. 26. Calvo poeta d'amore p. 28. L'elegia per Quintilia p. 31. Il poemetto Io p. 34 . Il frammento su Cerere p. 41. Frammenti di epitalamii p. 42. Altri frammenti p. 44.

Il. Il poeta Elvio Cinna . . . . .

La tradizione sulla tragica morte di Cinna p. 49. Le altre notizie su Cinna p. 54. Familiarità di Catullo e Cinna p. 57. Il poemetto Zmyrna p. 59. La Zmyrna e il poeta Ortensio p. 62. La Zmyrna, Volusio e il tumido Antimaco p. 66. L'oscurità 
della Zmyrna. Le testimonianze antiche in sua lode p. 72. Il mito di Smirna p. 75. I frammenti 1. 76. Ln epigramma di Cinna p. 78. Il Propermptiron Pollionis p. S0. Probabili frammenti del Propempticon p. 83. Altri frammenti delle poesie di Cinna p. Si.

III. II poeta Ansere.

pag. 93

Cinna ed Ansere nelle inenzioni di Ovidio e di Vergilio 1.95 . ali scherzi dei poeti sul nome $A u$ ser p. 96. Ansere e Catullo p. 97.

IV. Celio Rufo

I carmi di Catullo a Celio Rufo p. 101. Celio Rufo e Clodia p. 102. Il carme LXIX e il carme LXXVII di Catullo p. 103. Il sodalinio fraterno del carme C di Catullo p. 105. Il Lesbia nostra dell'epigramma Jitill a Celio p. $10 \mathrm{~s}$.

V. Ylamurra e Cesare. . . . . .

Il personaggio chiamato Mentula è Mamurra p. 113.

Le riechezze di Mamurra e la rivalità di Catullo j. 117. L'attendibilità delle accuse Catulliane contro Namurra p. His. Cesare chiamato cinedo komolo p. 121. Le altre invettive Catulliane contro Cesare p. I22. Le esagerazioni del poeta p. 123. L' epigramma allusion all' adulterio do Cesare con Mucia p. R.j. Riconciliazione con Cesare e celebrazione di lui p. 126.

vl. Lironia C'atulliana e l'epigramma a Cicerone. Carateristicle dell' ironia Catulliana p. I31. L' epigramma XXXXIX a Cicerme interpretato come seguo di devozione verso l' aratore p. 134. lnterpretato in senso ironico p. 136. Ragioni personali di acversione tra Cicerone e Catullo p. 139. Opposizione tra essi per diversita di rriterii artistici 1. 142.

Vll. C'atullo a Vrrona. Catullo a la sor ietia romana dell'etis silla

La memoria di Catullo, specialmente a Verona 0 nel Veneto, nell' antichiti e nel medio evo H. 147 . 
L' affetto di Catullo per Verona p. 154. Catullo a Roma p. 157. Amici e ricali di Catullo a Roma p. 159. I carmi per Giovenzio p. 167. I carmi contro Gellio p. 171. Le poesie amorose per Lesbia p. 174. Lesbia è Clodia p. 177. Clodio p. 185. Le fasi della grande passione amorosa di Catullo p. 186.

\section{APPENDICI}

I. Elementi rettorici nella poesia catulliana * . pag. 193

L' elucazione rettorica dei poeti romani p. 193. Il luogo comune geografico in Catullo p. 197. Il luogo comune mitologico p. 201. I luoghi comuni della poesia amorosa p. 206.

II. Orazio e Catullo ** . . . . . . . ” 211

Catullo e Calvo menzionati insieme da Orazio e da altri poeti p. 211. Imitazioni di Orazio da Catullo p. 214. Le ragioni dell' atteggiamento ostile di 0 razio verso Catullo p. 217.

* Dai Rendiconti del R. Istituto Lombardo di Scienze e Lettere, vol. XlviII, fasc. 9, Aprile 1915.

** Il saggio Orazio e Catullo. che vide primamente la luce nell'Athenaeum, studii periodici di letteratura e storia (Paria 1 Luglio 1915) è ripubblicato senza aggiunte o mutazioni; ma credo opportuno menzionare il lavoro di B. L. Ullmann, Horace, Catullus and Trgellius, in Classical P'hilology (July 1915, p. 270-296), che procede pero da criterii affutto clicersi. Prendo oecasione per arvertire che le pagine 10-14 dedicate all appellazione di doctus data ai poeti, sono comparse, con maggior copia di usserrazioni e di exempii, nel mio articolo Doctus Catullus in Athenaesm (Gennaio 1916, p. 1 segg.). 


\section{GIUNTE E CORREZIONI}

A p. VII crediamo opportuno aggiungere la menzione della editio minor curata da Robinson Ellis ne 1904: Catulli Carmina. recognovit brerique adnotatione eritica instruxit Romssox Encts. Uxonii, E typographeo Clarendoniano. - Ogni rolta però che nel nostro volume si trovano citati i Prolegomena dello Ellis, intendiamo riferirei alla edizione margiore: Catulli Teronensis liber, Oxonii, E typographeo Clarendoniano, 1878.

Voyliamo pur notato che dello Schwalie abbiamo usufruito non la prima edizione (Gissae, 1866), citata nella nota delle edizioni a pag. VIl, hensi la seconda (Berolini, Apud Weidmannos, 1886. igni volta che citiamo i Testimonia dello Schwabe, intendiamo riferirci a questa.

A pag. 149, linea $9^{\text {a }}$ della nota 1 , ove è stampato: p. $621 \mathrm{~B}$. ed. Ballerini, si corregga: p. 6i21, ed. Ballerini. 




\section{PLEASE DO NOT REMOVE CARDS OR SLIPS FROM THIS POCKET}

UNIVERSITY OF TORONTO LIBRARY 
